\title{
Transverse momentum in double parton scattering: factorisation, evolution and matching
}

\author{
Maarten G.A. Buffing, ${ }^{a}$ Markus Diehl ${ }^{a}$ and Tomas Kasemets ${ }^{b}$ \\ ${ }^{a}$ Deutsches Elektronen-Synchroton DESY, \\ Notkestraße 85, 22607 Hamburg, Germany \\ ${ }^{b}$ Nikhef Theory Group and VU University Amsterdam, \\ De Boelelaan 1081, 1081 HV Amsterdam, The Netherlands \\ E-mail: mbuffing@physics.ucla.edu, markus.diehl@desy.de, \\ kasemets@uni-mainz.de
}

ABSTRACT: We give a description of double parton scattering with measured transverse momenta in the final state, extending the formalism for factorisation and resummation developed by Collins, Soper and Sterman for the production of colourless particles. After a detailed analysis of their colour structure, we derive and solve evolution equations in rapidity and renormalisation scale for the relevant soft factors and double parton distributions. We show how in the perturbative regime, transverse momentum dependent double parton distributions can be expressed in terms of simpler nonperturbative quantities and compute several of the corresponding perturbative kernels at one-loop accuracy. We then show how the coherent sum of single and double parton scattering can be simplified for perturbatively large transverse momenta, and we discuss to which order resummation can be performed with presently available results. As an auxiliary result, we derive a simple form for the square root factor in the Collins construction of transverse momentum dependent parton distributions.

KeYwords: NLO Computations, QCD Phenomenology

ARXIV EPRINT: 1708.03528 


\section{Contents}

1 Introduction 1

2 Reminder: single TMD factorisation $\quad 4$

3 Defining double parton distributions $\quad \mathbf{5}$

$\begin{array}{lll}3.1 & \text { Collinear matrix elements } & 6\end{array}$

3.2 Soft factors 8

$\begin{array}{ll}3.3 & \text { Definition of DPDs } \\ & 13\end{array}$

$\begin{array}{lll}3.4 & \text { Renormalisation of DTMDs } & 14\end{array}$

$\begin{array}{ll}3.5 & \text { Definition of single parton TMDs } \\ \end{array}$

$\begin{array}{llr}4 & \text { Colour } & 17\end{array}$

$\begin{array}{lll}4.1 & \text { Projection operators } & 17\end{array}$

$\begin{array}{ll}4.2 & \text { Colour structure of the DPS cross section } 20\end{array}$

$\begin{array}{lll}4.3 & \text { Symmetry properties } & 21\end{array}$

4.4 Simplification of soft factors in collinear factorisation 24

4.5 Interlude: collinear factorisation for coloured particle production 26

5 Factorisation at small transverse momenta $\quad \mathbf{2 8}$

5.1 DTMD evolution: renormalisation scale and rapidity 30

$\begin{array}{lll}5.2 & \text { DPDF evolution } & 32\end{array}$

5.3 Combining DPS with SPS 34

$6 \quad$ Matching for small but perturbative transverse momenta 39

6.1 Regions of transverse momenta and distances 40

$\begin{array}{lll}6.2 & \text { The large- } \boldsymbol{y} \text { region } & 40\end{array}$

6.2.1 Short-distance expansion $\quad 41$

6.2.2 Evolution equations and their solution 43

6.2.3 Extrapolation to large $\boldsymbol{z}_{1}$ and $\boldsymbol{z}_{2} \quad 48$

$\begin{array}{lll}6.3 & \text { The small- } \boldsymbol{y} \text { region } & 49\end{array}$

$\begin{array}{lll}\text { 6.3.1 Short-distance expansion } & 49\end{array}$

6.3.2 Evolved DPDs and cross section $\quad 53$

6.4 Combining large and small $\boldsymbol{y} \quad 54$

6.5 Combining DPS with SPS at short distances 57

$\begin{array}{lll}6.6 & \text { Perturbative accuracy } & 60\end{array}$

7 One-loop results $\quad 64$

$\begin{array}{lll}\text { 7.1 TMD hard-scattering cross sections } & 64\end{array}$

$\begin{array}{lll}7.2 & \text { Collins-Soper kernels and anomalous dimensions } & 65\end{array}$

$\begin{array}{lll}\text { 7.2.1 Limit of small } \boldsymbol{z}_{1} \text { and } \boldsymbol{z}_{2} & 67\end{array}$

$\begin{array}{lll}7.3 & \text { Matching coefficients for DTMDs } & 68\end{array}$ 
7.3.1 General procedure

$\begin{array}{lll}\text { 7.3.2 Calculation of gluon-gluon matching coefficients } & 70\end{array}$

$\begin{array}{lll}\text { 7.3.3 Subtleties of renormalisation } & 75\end{array}$

$\begin{array}{lll}\text { 7.3.4 Matching coefficients for DTMDs on DPDFs } & 79\end{array}$

$\begin{array}{lll}\text { 7.3.5 Colour non-singlet channels } & 82\end{array}$

$\begin{array}{lll}7.4 & \text { Splitting kernels for DPDs } & 84\end{array}$

$\begin{array}{lll}8 & \text { Summary } & 85\end{array}$

$\begin{array}{ll}\text { A Matrix manipulations for the soft factor } & 88\end{array}$

$\begin{array}{ll}\text { B Comparison with the } \delta \text { regulator scheme } & 90\end{array}$

$\begin{array}{ll}\text { C Combining SPS and DPS for different hard scales } & 91\end{array}$

$\begin{array}{ll}\text { D Feynman rules } & 92\end{array}$

$\begin{array}{lll}\text { E Useful integrals } & 95\end{array}$

$\begin{array}{lll}\text { F } & \text { Tensor decomposition of DPDs } & 96\end{array}$

$\begin{array}{ll}\text { G Matching coefficients for (pseudo)scalar DPDs } & 97\end{array}$

$\begin{array}{ll}\text { H Splitting kernels for (pseudo)scalar DPDs } & 100\end{array}$

\section{Introduction}

Proton-proton collisions at high energies are sensitive to regions of phase space where partons have small momentum fractions. This implies high parton densities and thus increases the importance of events in which two or more partons in each proton take part in a hard interaction. The most frequent and best studied case of such multiple hard interactions is double parton scattering (DPS). This mechanism can be especially prominent in cross sections depending on transverse momenta in the final state. Its theoretical description involves double parton distributions (DPDs), which quantify the joint distribution of two partons inside a proton and contain a wealth of information on correlations between the proton constituents.

Experimental measurements of double parton scattering contributions to different final states dates back to experiments at the ISR [1] and SPS [2]. A wide range of DPS processes has been investigated at the Tevatron [3-11] and in run I of the LHC [12-21]. An overview of most of these measurements can be found in figure 14 of [19]. The importance of such processes will be even more pronounced at the full LHC energy (first results from run II are reported in [22]) and at future hadron colliders. 
Theoretical and phenomenological analyses of DPS have a long history; an overview of early work can be found in [23]. Following the seminal papers [24] and [25], significant effort has been invested in recent years to achieve a systematic theory description of DPS [2629], aiming at the same level of rigour as has been achieved for the familiar single parton scattering (SPS) mechanism. A formalism for combining the single and double parton scattering contributions to the physical cross section without double counting was recently presented in [30] — a different scheme was proposed earlier [31]. However, there remain several gaps in our understanding, and the present paper aims at closing some of them.

A crucial aspect for understanding DPS are correlations between the two partons that are probed in the reaction. Correlations in spin and in colour have been classified systematically $[27,28,32]$ and will play an important role in the present work. Their size is poorly known, but can be limited by positivity bounds [33, 34], which have similar theoretical status as positivity constraints on single parton distribution functions (PDFs). Quark model calculations [35-42] typically yield strong correlations in the valence region, but for the region of small momentum fractions $x$ there is little guidance from models so far. The decrease of spin correlations under evolution to higher scales has been studied in [43], and their influence on final-state distributions has been investigated for several processes $[28,44,45]$. The generation of parton correlations by the splitting of a single parton into two has been investigated by several groups [27, 46-50]; a simplified implementation into the event generator Pythia is described in [51, 52].

At the level of integrated cross sections, double parton scattering is suppressed by $\Lambda^{2} / Q^{2}$ compared to single parton scattering, where $Q$ denotes the scale of the hard scattering and $\Lambda$ the scale of nonperturbative interactions [26,53]. However, there are situations where DPS can nevertheless compete with SPS, for instance when the latter is suppressed by higher powers in coupling constants. A prominent example is same sign $W$ pair production [54-56], which is an important search channel for physics beyond the Standard Model. A generic mechanism enhancing DPS over SPS in processes involving small parton momentum fractions $x$ is the fact that with decreasing $x$ the density of two partons increases roughly like the square of the single parton density.

If the transverse momenta $q_{T}$ of the particles produced by a hard scattering are small compared with $Q$, then DPS has the same power behaviour in $1 / Q$ as SPS [26,53]. If $q_{T}$ is of order $\Lambda$, then one needs information about the nonperturbative "intrinsic" transverse momentum of partons. However, in the region $\Lambda \ll q_{T} \ll Q$ one can reliably compute the $q_{T}$ spectrum in perturbation theory, provided that one resums the large logarithms of $Q / q_{T}$ that arise in this regime. This resummation is intricately related with evolution in the rapidity of emitted gluons. For SPS processes producing colourless particles, like Drell-Yan lepton pairs or a Higgs boson, a powerful theoretical formalism has long been established $[57,58]$ and been pushed to high perturbative accuracy, see e.g. [59] and references therein. Formulations using soft-collinear effective theory (SCET) have been given in [60-62]. A precise theory for DPS at small measured $q_{T}$ is of obvious interest. In $[51,52]$ the DDT formalism [63] has been extended to DPS processes. The analysis in [26, 27] was based on transverse-momentum dependent (TMD) factorisation in the original formulation of Collins and Soper [64], which also underlies the CSS resummation formalism [57]. Since 
then, an improved version of TMD factorisation for SPS processes has been formulated by Collins [65]; a brief review is given in $[66,67]$ and the differences between the old and new versions are described in [68].

The aim of the present paper is twofold. Firstly, we complete the formulation of DPS in $[26,27]$ and adapt it to the new factorisation formalism of [65], providing a systematic analysis of soft-gluon effects, rapidity evolution and colour correlations. Secondly, we show how the theory simplifies for intermediate transverse momenta $\Lambda \ll q_{T} \ll Q$, where transverse-momentum dependent DPDs can be matched on transverse-momentum integrated distributions, with the transverse-momentum dependence being computed in perturbation theory. This significantly increases the predictive power of the theoretical framework. Some of our main results have been reported in $[69,70]$.

TMD factorisation in proton-proton collisions can be established to all orders in perturbation theory for the production of colourless particles such as a Higgs boson or electroweak gauge bosons [65]. Because of serious complications from soft gluon exchange, it is not known if and how the formalism could be extended to hard-scattering processes with coloured particles in the final state, such as jets or heavy quarks [71]. We will therefore limit our discussion of TMD factorisation in DPS to colourless final states as well. Important channels are the production of two electroweak gauge bosons (often called the double Drell-Yan process), of a Higgs boson and an electroweak gauge boson, or of a Higgs boson pair. Instead of a heavy boson, one may also consider a photon pair of large invariant mass.

This paper is organised as follows. In section 2 we recall some of the concepts and results for TMD factorisation in single parton scattering. In section 3 we discuss properties of collinear matrix elements and of the soft factor, which are the ingredients in the definition of transverse-momentum dependent and transverse-momentum integrated DPDs, which we will call DTMDs and DPDFs, respectively. Our definition generalises the combination of collinear and soft factors in [65] to double parton distributions, and it provides an alternative form of this construction for single parton TMDs. The colour structure of DPS is significantly more complicated than the one of SPS, and we show in section 4 how this structure can be handled in a general and efficient way. We find significant simplifications for transverse-momentum integrated quantities. In section 5 we present the general factorisation formula for DPS at low $q_{T}$, its combination with SPS, as well as the evolution equations for DTMDs and DPDFs and their general solution. Section 6 is devoted to the region $\Lambda \ll q_{T} \ll Q$. We establish the matching of DTMDs onto different types of transverse-momentum integrated distributions. The multi-scale nature of the problem leads to different matching regimes, which we combine in a consistent way using a subtraction formalism. In section 7 we give one-loop expressions for perturbative quantities that appear in the DPS cross section, extending previous work in the literature and discussing several technical aspects of the computation. We summarise our main results in section 8. A variety of technical details and results are given in the appendices. 


\section{Reminder: single TMD factorisation}

To begin with, let us recall a few results from TMD factorisation, as laid out in [65]. The cross section depends on TMDs that describe the distribution of partons inside the proton in both longitudinal and transverse momentum. Throughout this work, we consider unpolarised protons. The factorisation formula for Drell-Yan production then reads

$$
\frac{d \sigma}{d x d \bar{x} d^{2} \boldsymbol{q}}=\sum_{q} \hat{\sigma}_{q \bar{q}}(Q, \mu) \int \frac{d^{2} \boldsymbol{z}}{(2 \pi)^{2}} e^{-i \boldsymbol{q} \boldsymbol{z}} W_{q \bar{q}}(x, \bar{x}, \boldsymbol{z} ; \mu)+\{q \leftrightarrow \bar{q}\},
$$

where $\hat{\sigma}_{q \bar{q}}$ is the cross section for $q \bar{q}$ annihilation into an electroweak gauge boson and

$$
W_{q \bar{q}}(x, \bar{x}, \boldsymbol{z} ; \mu)=f_{q}(x, \boldsymbol{z} ; \mu, \zeta) f_{\bar{q}}(\bar{x}, \boldsymbol{z} ; \mu, \bar{\zeta})
$$

is the product of a quark and an antiquark TMD. The invariant mass of the boson is $Q$ and its transverse momentum is $\boldsymbol{q}$. The TMDs depend on longitudinal momentum fractions $(x$ or $\bar{x}$ ) that are fixed by final-state kinematics, and on a transverse distance $\boldsymbol{z}$ that is Fourier conjugate to the transverse parton momentum (and often denoted by $\boldsymbol{b}$ in the literature). They also depend on an ultraviolet renormalisation scale $\mu$ and on a rapidity parameter $(\zeta$ or $\bar{\zeta})$ as we will review later. Notice that the rapidity parameter dependence cancels in $W_{q \bar{q}}$. The parton-level cross section $\hat{\sigma}_{q \bar{q}}$ and the overall cross section may be taken differential in additional variables if more than one particle is produced in the hard scattering. An example is the angular distribution of the leptons into which the electroweak gauge boson decays in Drell-Yan production. In this case one must include the TMDs for transverse quark and antiquark polarisation in (2.2).

The dependence of a TMD on the renormalisation scale is given by $[65,66]$

$$
\frac{\partial}{\partial \log \mu} f_{a}(x, \boldsymbol{z} ; \mu, \zeta)=\gamma_{F, a}(\mu, \zeta) f_{a}(x, \boldsymbol{z} ; \mu, \zeta),
$$

where $a=q, \bar{q}, g$ labels the parton type. The rapidity dependence of the anomalous dimension $\gamma_{F, a}$ is given by

$$
\frac{\partial}{\partial \log \zeta} \gamma_{F, a}(\mu, \zeta)=-\frac{1}{2} \gamma_{K, a}(\mu),
$$

where $\gamma_{K, a}$ is called the cusp anomalous dimension. It depends on $\mu$ via $\alpha_{s}(\mu)$, i.e. $\gamma_{K, a}(\mu)=$ $\gamma_{K, a}\left(\alpha_{s}(\mu)\right)$. From $(2.4)$ one readily finds

$$
\gamma_{F, a}(\mu, \zeta)=\gamma_{a}(\mu)-\gamma_{K, a}(\mu) \log \frac{\sqrt{\zeta}}{\mu}
$$

with

$$
\gamma_{a}(\mu)=\gamma_{F, a}\left(\mu, \mu^{2}\right)
$$

The evolution of TMDs with the rapidity scale $\zeta$ is governed by the Collins-Soper equation ${ }^{1}$

$$
\frac{\partial}{\partial \log \zeta} f_{a}(x, \boldsymbol{z} ; \mu, \zeta)=\frac{1}{2} K_{a}(\boldsymbol{z} ; \mu) f_{a}(x, \boldsymbol{z} ; \mu, \zeta),
$$

\footnotetext{
${ }^{1}$ We generally follow the notation of $[65,68]$ in the present paper. We do however not use a tilde to denote quantities in transverse position space, thus writing $f$ and $K$ instead of $\tilde{f}$ and $\tilde{K}$.
} 
whose kernel satisfies

$$
\frac{\partial}{\partial \log \mu} K_{a}(z ; \mu)=-\gamma_{K, a}(\mu)
$$

If the transverse boson momentum $\boldsymbol{q}$ is large compared with the scale $\Lambda$ of non-perturbative interactions, one can use a short-distance expansion that connects a TMD $f_{a}(x, \boldsymbol{z})$ at small $\boldsymbol{z}$ with a conventional collinear $\mathrm{PDF} f_{b}(x)$ according to

$$
f_{a}(x, \boldsymbol{z} ; \mu, \zeta)=\sum_{b} C_{a b}\left(x^{\prime}, \boldsymbol{z} ; \mu, \zeta\right) \underset{x}{\otimes} f_{b}\left(x^{\prime} ; \mu\right)
$$

with the convolution product defined by

$$
C\left(x^{\prime}\right) \underset{x}{\otimes} f\left(x^{\prime}\right)=\int_{x}^{1} \frac{d x^{\prime}}{x^{\prime}} C\left(x^{\prime}\right) f\left(\frac{x}{x^{\prime}}\right) .
$$

The expansion (2.9) has power corrections in the parameter $\Lambda|\boldsymbol{z}|$. Combining it with the solution of the evolution equations in $\mu$ and $\zeta$, one obtains

$$
\begin{aligned}
f_{a}(x, \boldsymbol{z} ; \mu, \zeta)= & \exp \left\{\int_{\mu_{0}}^{\mu} \frac{d \mu^{\prime}}{\mu^{\prime}}\left[\gamma_{a}\left(\mu^{\prime}\right)-\gamma_{K, a}\left(\mu^{\prime}\right) \log \frac{\sqrt{\zeta}}{\mu^{\prime}}\right]+K_{a}\left(\boldsymbol{z} ; \mu_{0}\right) \log \frac{\sqrt{\zeta}}{\mu_{0}}\right\} \\
& \times \sum_{b} C_{a b}\left(x^{\prime}, \boldsymbol{z} ; \mu_{0}, \mu_{0}^{2}\right) \underset{x}{\otimes} f_{b}\left(x^{\prime} ; \mu_{0}\right) .
\end{aligned}
$$

The short-distance coefficient $C$ and the Collins-Soper kernel $K$ should be evaluated with a scale choice that avoids large logarithms, so that they can be reliably calculated in fixedorder perturbation theory. In the non-perturbative region of $\boldsymbol{z}$ one needs a model ansatz for $f_{a}(x, \boldsymbol{z})$. The so-called $b^{*}$ prescription $[57,72]$ can be used to smoothly interpolate between such an ansatz and the perturbative result (2.11).

In the following sections, we will show how these results can be extended to the case of double parton scattering. There are several aspects that make this extension far from trivial. One is the larger number of coloured particles involved in the process, which leads to a non-trivial colour structure of DTMDs and DPDFs. As a consequence, even DPDFs depend on the rapidity parameter $\zeta$, unlike PDFs for a single parton [27, 28]. A second aspect is that DPS involves several transverse distances, which makes the analogue of the short-distance expansion (2.9) more complicated.

\section{Defining double parton distributions}

As reviewed in [29], factorisation of DPS processes involves separating the leading graphs for the cross sections into subgraphs that are hard, soft, or collinear to one of the two incoming protons. The treatment of the soft subgraph is intimately related with the rapidity parameter $\zeta$ mentioned in the previous subsection.

There are in fact different alternatives for such a treatment. The analysis of the double Drell-Yan process in [27] followed the original procedure for TMD factorisation by Collins and Soper [64] and did not work out all relevant aspects of the problem. In the present paper, we perform a systematic analysis of DPS in the factorisation framework of Collins [65]. We explicitly show how the soft factors relevant for the cross section can be 
entirely absorbed into DTMDs or DPDFs, and we derive the resulting evolution equations in $\zeta$, as well as the ones in $\mu$. Other schemes to handle soft factors and rapidity dependence will briefly be discussed in section 8 .

The starting point of our discussion is an intermediate expression of the DPS cross section, given in section 2.1 of [29]. The cross section for the production of two sets of colourless particles involves a term

$$
H_{1, q \bar{q}} H_{2, q \bar{q}} F_{\mathrm{us}, \bar{q} \bar{q}}^{T}\left(v_{R}\right) S_{q q}^{-1}\left(v_{L}, v_{R}\right) S_{q q}\left(v_{L}, v_{R}\right) S_{q q}^{-1}\left(v_{L}, v_{R}\right) F_{\mathrm{us}, q q}\left(v_{L}\right)
$$

for the annihilation of two quarks in one proton with two antiquarks in the other proton, and corresponding terms for the other parton combinations. For definiteness we have only written down the DPDs for unpolarised partons; polarised terms have the same soft factor. $H_{i, q \bar{q}}$ denotes the squared hard-scattering amplitudes, with appropriate spin projections (see section 2.2 in [27]) but with the colour structure removed as specified in (4.55) below. $F_{\text {us }}$ denotes unsubtracted collinear matrix elements, and $S$ is a soft factor. The inverse of this factor removes contributions of soft gluons from the unsubtracted collinear matrix elements, so that $S^{-1} F_{\text {us }}$ receives only contributions from collinear gluons. For brevity we have omitted momentum fraction and position space arguments in (3.1), as well as renormalisation and factorisation scales and colour indices. $F_{\mathrm{us}}$ is a row vector in colour space (with one index for each of the four parton legs), and $S$ is a matrix with two times four indices. The spacelike four-vectors $v_{L}$ and $v_{R}$ denote the directions of Wilson lines and will be specified later. As discussed in $[29,65], v_{L}$ and $v_{R}$ have to be chosen such that the effects of so-called Glauber gluon exchange on the cross section can be subsumed into the soft and collinear factors in (3.1).

The aim of the following sections is to combine soft factors and collinear matrix elements in such a way that the product (3.1) takes on a simple form. After introducing the necessary notation, we derive a number of symmetry properties of soft factors in section 3.2 and then discuss our central hypothesis for their rapidity dependence, given in (3.24) and (3.25). This leads us to the definition (3.29) of DPDs and to their rapidity evolution equation (3.34) in section 3.3. We discuss renormalisation in section 3.4 and derive the basic evolution equations (3.43) and (3.44) relevant for DTMDs. Applying our construction to TMDs for a single parton in section 3.5, we are led to the definition (3.49) and see that it is equivalent to the definition of Collins [65] by virtue of the relation (3.48).

\subsection{Collinear matrix elements}

To begin with, let us recall the definitions of unsubtracted DTMDs and DPDFs in terms of proton matrix elements. These will later be combined with soft factors in order to define the double parton distributions that appear in the cross section formula.

For two partons $a_{1}$ and $a_{2}$, the unsubtracted DTMDs are defined in terms of matrix elements as [26, 27]

$$
\begin{aligned}
F_{\mathrm{us}, a_{1} a_{2}}\left(x_{1}, x_{2}, \boldsymbol{z}_{1}, \boldsymbol{z}_{2}, \boldsymbol{y}\right)= & 2 p^{+}\left(x_{1} p^{+}\right)^{-n_{1}}\left(x_{2} p^{+}\right)^{-n_{2}} \int \frac{d z_{1}^{-}}{2 \pi} \frac{d z_{2}^{-}}{2 \pi} d y^{-} e^{i\left(x_{1} z_{1}^{-}+x_{2} z_{2}^{-}\right) p^{+}} \\
& \times\left\langle p\left|\mathcal{O}_{a_{1}}\left(y, z_{1}\right) \mathcal{O}_{a_{2}}\left(0, z_{2}\right)\right| p\right\rangle
\end{aligned}
$$


where $n_{i}=1$ if parton number $i$ is a gluon and $n_{i}=0$ otherwise. We use light-cone coordinates $w^{ \pm}=\left(w^{0} \pm w^{3}\right) / \sqrt{2}$ and the transverse component $\boldsymbol{w}=\left(w^{1}, w^{2}\right)$ for any fourvector $w$. The definition (3.2) is natural for a proton moving to the right, i.e. for $p^{3}>0$. For a left moving proton, i.e. for $p^{3}<0$, one would interchange the roles of plus and minus coordinates. It is understood that $\boldsymbol{p}=\mathbf{0}$ in both cases, and that the proton polarisation is averaged over. Setting $\boldsymbol{z}_{1}=\boldsymbol{z}_{2}=\mathbf{0}$ in (3.2), one obtains DPDFs, which are relevant for collinear factorisation. As we will discuss later, this changes the ultraviolet behaviour of the operators.

The operators for quarks in a right moving proton read

$$
\mathcal{O}_{a}(y, z)=\left.\bar{q}\left(y-\frac{1}{2} z\right) W^{\dagger}\left(y-\frac{1}{2} z, v_{L}\right) \Gamma_{a} W\left(y+\frac{1}{2} z, v_{L}\right) q\left(y+\frac{1}{2} z\right)\right|_{z^{+}=y^{+}=0}
$$

with spin projections

$$
\Gamma_{q}=\frac{1}{2} \gamma^{+}, \quad \Gamma_{\Delta q}=\frac{1}{2} \gamma^{+} \gamma_{5}, \quad \Gamma_{\delta q}^{j}=\frac{1}{2} i \sigma^{j+} \gamma_{5} \quad(j=1,2)
$$

onto unpolarised quarks $(q)$, longitudinally polarised quarks $(\Delta q)$ and transversely polarised quarks $(\delta q)$. We do not explicitly display the transverse index $j$ of the operator $\mathcal{O}_{\delta q}$ and of the corresponding DPDs, unless it is needed. The field with argument $y+\frac{1}{2} z$ in $\mathcal{O}_{q}(y, z)$ is associated with a quark in the amplitude of a double scattering process and the field with argument $y-\frac{1}{2} z$ with a quark in the complex conjugate amplitude. The Wilson lines are defined as

$$
W(\xi, v)=\mathrm{P} \exp \left[i g t^{a} \int_{-\infty}^{0} d s v A^{a}(\xi+s v)\right]
$$

where $\mathrm{P}$ denotes path ordering, such that fields $t^{a} A^{a}(\xi+s v)$ with smaller $s$ stand further to the left in the expanded exponential. Our convention for the strong coupling $g$ is specified in appendix D. Throughout this work, we only consider the case $\boldsymbol{v}=\mathbf{0}$. In the matrix element (3.2) for a right-moving proton, one takes a direction $v_{L}$ with $v_{L}^{-} \gg-v_{L}^{+}>0$, and in its analogue for a left-moving proton one has a direction $v_{R}$ with $v_{R}^{+} \gg-v_{R}^{-}>0$. In both cases, the Wilson lines are past-pointing. Analogous operators are defined for antiquarks, with some sign changes as specified in section 2.2 of [27]. For gluons, one has

$$
\mathcal{O}_{a}(y, z)=\left.\Pi_{a}^{j j^{\prime}} G^{+j^{\prime}}\left(y-\frac{1}{2} z\right) W^{\dagger}\left(y-\frac{1}{2} z, v_{L}\right) W\left(y+\frac{1}{2} z, v_{L}\right) G^{+j}\left(y+\frac{1}{2} z\right)\right|_{z^{+}=y^{+}=0}
$$

with spin projections

$$
\Pi_{g}^{j j^{\prime}}=\delta^{j j^{\prime}}, \quad \quad \Pi_{\Delta g}^{j j^{\prime}}=i \epsilon^{j j^{\prime}}, \quad\left[\Pi_{\delta g}^{k k^{\prime}}\right]^{j j^{\prime}}=\tau^{j j^{\prime}, k k^{\prime}}
$$

onto unpolarised gluons $(g)$, longitudinally polarised gluons $(\Delta g)$ and linearly polarised gluons $(\delta g)$. The indices $j, j^{\prime}, k, k^{\prime}=1,2$ run over transverse components, $\epsilon^{j j^{\prime}}$ is the antisymmetric tensor with $\epsilon^{12}=1$, and $\tau^{j j^{\prime}, k k^{\prime}}$ is defined as

$$
\tau^{j j^{\prime}, k k^{\prime}}=\frac{1}{2}\left(\delta^{j k} \delta^{j^{\prime} k^{\prime}}+\delta^{j k^{\prime}} \delta^{j^{\prime} k}-\delta^{j j^{\prime}} \delta^{k k^{\prime}}\right)
$$


The Wilson lines in (3.6) are in the adjoint representation rather than in the fundamental one (see section 3.2). Making the colour indices of the operators explicit, we have

$$
\mathcal{O}_{q, j j^{\prime}}=\bar{q}_{k^{\prime}}\left(W^{\dagger}\right)_{k^{\prime} j^{\prime}} \Gamma W_{j k} q_{k}, \quad \mathcal{O}_{g, a a^{\prime}}=\Pi G_{b^{\prime}}\left(W^{\dagger}\right)_{b^{\prime} a^{\prime}} W_{a b} G_{b}
$$

for quarks and gluons, respectively.

For the discussion of ultraviolet renormalisation and of the short-distance expansion, it is useful to introduce operators that correspond to a definite light-cone momentum fraction $x$ of a parton:

$$
O_{a}(x, \boldsymbol{y}, \boldsymbol{z})=2 p^{+}\left(x p^{+}\right)^{-n} \int \frac{d z^{-}}{2 \pi} d y^{-} e^{i x z^{-} p^{+}} \mathcal{O}_{a}(y, z) .
$$

Using translation invariance, one readily finds that unsubtracted single and double parton TMDs are then given by matrix elements

$$
\begin{aligned}
2 \pi \delta\left(p^{+}-p^{+}\right) 2 p^{+} f_{\mathrm{us}, a}(x, \boldsymbol{z}) & =\left\langle p^{\prime}\left|O_{a}(x, \mathbf{0}, \boldsymbol{z})\right| p\right\rangle, \\
2 \pi \delta\left(p^{+}-p^{+}\right) 2 p^{+} F_{\mathrm{us}, a_{1} a_{2}}\left(x_{1}, x_{2}, \boldsymbol{z}_{1}, \boldsymbol{z}_{2}, \boldsymbol{y}\right) & =\left\langle p^{\prime}\left|O_{a_{1}}\left(x_{1}, \boldsymbol{y}, \boldsymbol{z}_{1}\right) O_{a_{2}}\left(x_{2}, \mathbf{0}, \boldsymbol{z}_{2}\right)\right| p\right\rangle,
\end{aligned}
$$

where $\boldsymbol{p}^{\prime}=\boldsymbol{p}=\mathbf{0}$. The corresponding collinear distributions are obtained by setting $\boldsymbol{z}, \boldsymbol{z}_{1}$ and $z_{2}$ to zero.

We note that in the DPS cross section, there are also distributions describing the interference between different parton types in the process amplitude and its complex conjugate, i.e. between quarks and antiquarks, between quarks and gluons, or between quarks of different flavour $[27,28,33,34]$. At low values of $x_{1}$ and $x_{2}$, such interference DPDs are expected to be negligible, because they have no dynamic cross talk with gluon distributions, which grow most strongly with decreasing $x$. Although we do not consider interference DPDs in this work, they can be treated with the methods presented in the following.

Let us remark that an analysis of transverse-momentum dependent DPDs in the small$x$ limit has recently been given in [73]. The quantities considered in that work are Fourier transformed w.r.t. our variable $\boldsymbol{z}_{i}$ and - more importantly - integrated over $\boldsymbol{y}$. To make contact with the DPS cross section, one would need to restore the dependence of these distributions on $\boldsymbol{y}$, or on the Fourier conjugated momentum.

\subsection{Soft factors}

Before constructing the final DPDs, we must take a closer look at the soft factor, which is defined as the vacuum expectation value of Wilson lines. For reasons that will become clear later, we define an "extended soft factor" with open colour indices of all Wilson lines as

$$
\begin{aligned}
& {\left[S_{q q}\left(\boldsymbol{z}_{1}, \boldsymbol{z}_{2}, \boldsymbol{y} ; v_{L}, v_{R}\right)\right]_{j_{1} j_{1}^{\prime} j_{2} j_{2}^{\prime}, k_{1} k_{1}^{\prime} k_{2} k_{2}^{\prime}}^{i_{1} i_{1}^{\prime} i_{2}^{\prime} l_{2}, l_{1}^{\prime} l_{2} l_{2}^{\prime}}} \\
& \quad=\left\langle 0\left|\left[O_{S, q}\left(\boldsymbol{y}, \boldsymbol{z}_{1} ; v_{L}, v_{R}\right)\right]_{j_{1} j_{1}^{\prime}, k_{1} k_{1}^{\prime}}^{i_{1} i_{1}^{\prime}, l_{1} l_{1}^{\prime}}\left[O_{S, q}\left(\mathbf{0}, \boldsymbol{z}_{2} ; v_{L}, v_{R}\right)\right]_{j_{2} j_{2}^{\prime}, k_{2} k_{2}^{\prime}}^{i_{2} i_{2}^{\prime}, l_{2} l_{2}^{\prime}}\right| 0\right\rangle .
\end{aligned}
$$

This factor appears in the cross section with two $q \bar{q}$ annihilation subprocesses, and the subscripts $q$ refer to the right-moving parton in each subprocess. The operator

$$
\begin{aligned}
& {\left[O_{S, q}\left(\boldsymbol{y}, \boldsymbol{z} ; v_{L}, v_{R}\right)\right]_{j j^{\prime}, k k^{\prime}}^{i i^{\prime}, l l^{\prime}}} \\
& \quad=W_{i j}\left(\boldsymbol{y}+\frac{1}{2} \boldsymbol{z}, v_{L}\right) W_{k l}^{\dagger}\left(\boldsymbol{y}+\frac{1}{2} \boldsymbol{z}, v_{R}\right) W_{l^{\prime} k^{\prime}}\left(\boldsymbol{y}-\frac{1}{2} \boldsymbol{z}, v_{R}\right) W_{j^{\prime} i^{\prime}}^{\dagger}\left(\boldsymbol{y}-\frac{1}{2} \boldsymbol{z}, v_{L}\right)
\end{aligned}
$$



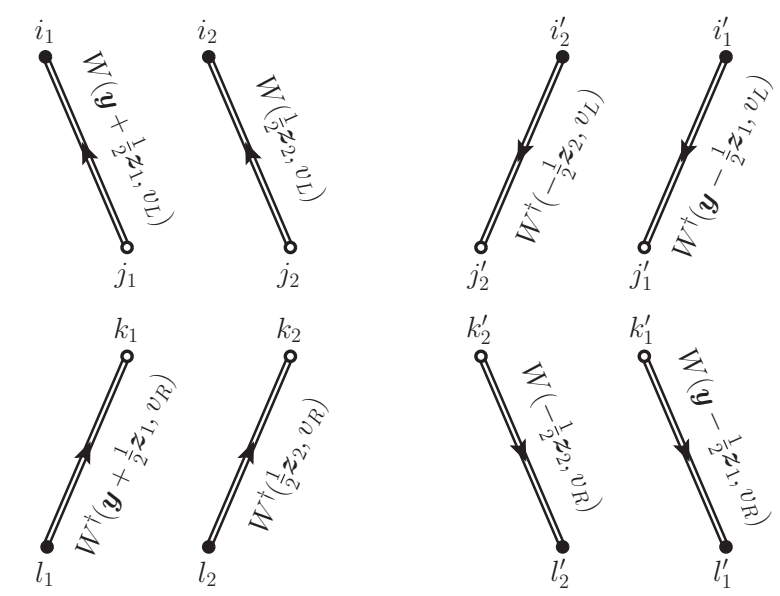

Figure 1. Wilson lines and colour indices of the extended soft factor defined by (3.12) and (3.13). Wilson lines with subscripts 1 are grouped into the operator $O_{S, q}\left(\boldsymbol{y}, \boldsymbol{z}_{1}\right)$ and those with subscripts 2 into the operator $O_{S, q}\left(\mathbf{0}, \boldsymbol{z}_{2}\right)$.

is a product of four Wilson lines, which are defined as in (3.5), but with $\xi^{+}=\xi^{-}=0$, so that their position arguments are only in the transverse plane and hence written in boldface. Primed and unprimed indices $j$ and $k$ in the Wilson lines of (3.13) correspond to gluon fields $A(\xi)$ at light-cone zero $\left(\xi^{+}=\xi^{-}=0\right)$, whereas primed and unprimed indices $i$ and $l$ correspond to gluon fields at light-cone infinity, $A(\xi-\infty v)$. A pictorial representation of (3.12) is given in figure 1.

For right-moving antiquarks instead of quarks, one replaces $W_{i j}$ with $W_{i j}^{*}=W_{j i}^{\dagger}$ and vice versa, which corresponds to replacing $i t_{i j}^{a}$ with $\left(i t_{i j}^{a}\right)^{*}=-i t_{j i}^{a}$ in the exponential (3.5). For gluons, one takes adjoint Wilson lines $W_{b c}$, obtained by replacing $i t_{i j}^{a}$ in (3.5) with $f^{a b c}$. This reflects the fact that the generators $T^{a}$ of the colour group in the adjoint representation are given by $\left(T^{a}\right)_{b c}=-i f^{a b c}$. We therefore have

$$
S_{q q}=S_{\bar{q} \bar{q}}^{*}, \quad S_{q \bar{q}}=S_{\bar{q} q}^{*}, \quad S_{q g}=S_{\bar{q} g}^{*}, \quad S_{g g}=S_{g g}^{*},
$$

where we have omitted colour indices for brevity. Note that, by construction, the Wilson line operators for all representations are unitary, i.e. $W(\xi, v) W^{\dagger}(\xi, v)=\mathbb{1}$, where $\mathbb{1}$ is the unit matrix in the relevant colour space.

In processes producing colourless particles, one needs (3.12) with all index pairs $j, k$ contracted, i.e.

$$
\begin{aligned}
& {\left[S_{q q}\left(\boldsymbol{z}_{1}, \boldsymbol{z}_{2}, \boldsymbol{y} ; v_{L}, v_{R}\right)\right]^{i_{1} i_{1}^{\prime} i_{2} i_{2}^{\prime}, l_{1} l_{1}^{\prime} l_{2} l_{2}^{\prime}}} \\
& \quad=\left\langle 0\left|\left[O_{S, q}\left(\boldsymbol{y}, \boldsymbol{z}_{1} ; v_{L}, v_{R}\right)\right]^{i_{1} i_{1}^{\prime}, l_{1} l_{1}^{\prime}}\left[O_{S, q}\left(\mathbf{0}, \boldsymbol{z}_{2} ; v_{L}, v_{R}\right)\right]^{i_{2} i_{2}^{\prime}, l_{2} l_{2}^{\prime}}\right| 0\right\rangle
\end{aligned}
$$

with

$$
\begin{aligned}
& {\left[O_{S, q}\left(\boldsymbol{y}, \boldsymbol{z} ; v_{L}, v_{R}\right)\right]^{i i^{\prime}, l l^{\prime}}} \\
& \quad=\left[W\left(\boldsymbol{y}+\frac{1}{2} \boldsymbol{z}, v_{L}\right) W^{\dagger}\left(\boldsymbol{y}+\frac{1}{2} \boldsymbol{z}, v_{R}\right)\right]_{i l}\left[W\left(\boldsymbol{y}-\frac{1}{2} \boldsymbol{z}, v_{R}\right) W^{\dagger}\left(\boldsymbol{y}-\frac{1}{2} \boldsymbol{z}, v_{L}\right)\right]_{l^{\prime} i^{\prime}}
\end{aligned}
$$


We simply call this the "soft factor" (without the specification "extended"). Regarding this as a matrix in the index pairs $\left(i_{1} i_{1}^{\prime} i_{2} i_{2}^{\prime}\right)$ and $\left(l_{1} l_{1}^{\prime} l_{2} l_{2}^{\prime}\right)$, we define the transposed matrix as

$$
\left[S_{q q}^{T}\left(\boldsymbol{z}_{1}, \boldsymbol{z}_{2}, \boldsymbol{y} ; v_{L}, v_{R}\right)\right]^{i_{1} i_{1}^{\prime} i_{2} i_{2}^{\prime}, l_{1} l_{1}^{\prime} l_{2} l_{2}^{\prime}}=\left[S_{q q}\left(\boldsymbol{z}_{1}, \boldsymbol{z}_{2}, \boldsymbol{y} ; v_{L}, v_{R}\right)\right]^{l_{1} l_{1}^{\prime} l_{2} l_{2}^{\prime}, i_{1} i_{1}^{\prime} i_{2} i_{2}^{\prime}}
$$

and the Hermitian conjugate as $S_{q q}^{\dagger}=\left[S_{q q}^{T}\right]^{*}$. Corresponding definitions hold for $S_{g g}, S_{g q}$, $S_{\bar{q} \bar{q}}$ etc. There are a number of symmetry constraints on the soft factor:

1. With the restrictions on the directions of Wilson lines in $S\left(v_{L}, v_{R}\right)$ specified below (3.5), one can always perform a longitudinal boost such that $v_{L}=(\alpha, \beta, \mathbf{0})$ and $v_{R}=(\beta, \alpha, \mathbf{0})$. A parity transformation interchanges plus- and minus components and thus, combined with the boost, exchanges $v_{L} \leftrightarrow v_{R}$. Parity and boost invariance therefore give

$$
\begin{aligned}
S_{a_{1} a_{2}}\left(\boldsymbol{z}_{1}, \boldsymbol{z}_{2}, \boldsymbol{y} ; v_{L}, v_{R}\right) & =S_{a_{1} a_{2}}\left(-\boldsymbol{z}_{1},-\boldsymbol{z}_{2},-\boldsymbol{y} ; v_{R}, v_{L}\right) \\
& =S_{a_{1} a_{2}}\left(\boldsymbol{z}_{1}, \boldsymbol{z}_{2}, \boldsymbol{y} ; v_{R}, v_{L}\right)
\end{aligned}
$$

where $a_{1}, a_{2}$ denote the parton types, $q, \bar{q}, g$. In the final step we used that the soft factor depends on position arguments only via scalar products between $\boldsymbol{z}_{1}, \boldsymbol{z}_{2}$ and $\boldsymbol{y}$. This follows from rotational and parity invariance, combined with the fact that the Wilson line directions $v_{L}$ and $v_{R}$ are purely longitudinal.

2. A combined parity and time reversal transformation reverses the vectors $v_{L}$ and $v_{R}$, as well as the position arguments. This gives

$$
\begin{aligned}
S_{a_{1} a_{2}}\left(\boldsymbol{z}_{1}, \boldsymbol{z}_{2}, \boldsymbol{y} ; v_{L}, v_{R}\right) & =S_{a_{1} a_{2}}\left(-\boldsymbol{z}_{1},-\boldsymbol{z}_{2},-\boldsymbol{y} ;-v_{L},-v_{R}\right) \\
& =S_{a_{1} a_{2}}\left(\boldsymbol{z}_{1}, \boldsymbol{z}_{2}, \boldsymbol{y} ;-v_{L},-v_{R}\right),
\end{aligned}
$$

where in the last step we used the same symmetry argument as in (3.18).

3. Since the soft factor is constructed from products $\left[W\left(z, v_{L}\right) W^{\dagger}\left(z, v_{R}\right)\right]_{i l}$ for quarks, $\left[W\left(z, v_{R}\right) W^{\dagger}\left(z, v_{L}\right)\right]_{l i}$ for antiquarks, and $\left[W\left(z, v_{L}\right) W^{\dagger}\left(z, v_{R}\right)\right]_{a d}$ for gluons, we have

$$
\begin{aligned}
S_{a_{1} a_{2}}\left(\boldsymbol{z}_{1}, \boldsymbol{z}_{2}, \boldsymbol{y} ; v_{L}, v_{R}\right) & =S_{a_{1} a_{2}}^{\dagger}\left(\boldsymbol{z}_{1}, \boldsymbol{z}_{2}, \boldsymbol{y} ; v_{R}, v_{L}\right) \\
& =S_{a_{1} a_{2}}^{\dagger}\left(\boldsymbol{z}_{1}, \boldsymbol{z}_{2}, \boldsymbol{y} ; v_{L}, v_{R}\right)
\end{aligned}
$$

where in the second step we have used (3.18). The soft matrix is thus Hermitian in the groups of indices for the Wilson lines along the different directions $v_{L}$ and $v_{R}$. Combining this result with the relations (3.14), we find

$$
S_{q q}=S_{\bar{q} \bar{q}}^{T}, \quad S_{q \bar{q}}=S_{\bar{q} q}^{T}, \quad S_{q g}=S_{\bar{q} g}^{T}, \quad S_{g g}=S_{g g}^{T},
$$

where the matrices on the left and the right hand side have identical arguments. We can thus identify the product $F_{\mathrm{us}, \bar{q} \bar{q}}^{T} S_{q q}^{-1}=\left(S_{\bar{q} \bar{q}}^{-1} F_{\mathrm{us}, \bar{q} \bar{q}}\right)^{T}$ in the expression (3.1) as the analogue of the product $S_{q q}^{-1} F_{\mathrm{us}, q q}$. 
From now on we restrict ourselves to spacelike Wilson line directions in $S\left(v_{L}, v_{R}\right)$ that satisfy

$$
v_{L}^{-}>0, \quad v_{R}^{+}>0, \quad Y_{L} \ll Y_{R},
$$

where the rapidity $Y_{v}$ of the spacelike vector $v$ is defined as

$$
Y_{v}=\frac{1}{2} \log \left|\frac{v^{+}}{v^{-}}\right| .
$$

Cases other than (3.22), such as $v_{L}^{-}<0, v_{R}^{+}<0$, can be realised using the symmetry relations (3.18) and (3.19). Owing to boost invariance and the fact that the Wilson lines $W(\xi, v)$ are invariant under rescaling $v \rightarrow \lambda v$, the soft factor depends on $v_{L}$ and $v_{R}$ only via the difference of the Wilson line rapidities. We can hence write $S\left(v_{L}, v_{R}\right)=S\left(Y_{R}-Y_{L}\right)$.

At this point we make an assumption on the properties of the soft factor, which we cannot fully prove (we specify below what can be proven at present).

For the soft factor $S\left(Y_{R}-Y_{L}\right)$ with $Y_{R}-Y_{L} \gg 1$ (for brevity we omit the indices $a_{1}$, $a_{2}$ and arguments $\left.\boldsymbol{z}_{1}, \boldsymbol{z}_{2}, \boldsymbol{y}\right)$, we assume that there exists a matrix function $s(Y)$ satisfying the following three properties:

Property 1a: The rapidity dependence is given by

$$
\frac{\partial}{\partial Y} s(Y)=s(Y) K
$$

with $K=K^{\dagger}$ independent of $Y$.

Property 1b: $s(Y)$ is nonsingular. It is sufficient to establish this property at an arbitrary value $Y_{1}$; its validity at any other $Y$ then follows from the solution of (3.24).

Property 1c: One has

$$
S(Y)=s\left(Y-Y_{0}\right) s^{\dagger}\left(Y_{0}\right) \quad \text { for } Y \gg 1 \text { and arbitrary } Y_{0} .
$$

It is easy to see that $s(Y)$ is not unique, since properties 1a-1c remain true if one redefines $s(Y) \rightarrow s(Y) U$ and $K \rightarrow U^{\dagger} K U$ with a $Y$ independent unitary matrix $U$. Conversely, if properties 1a-1c hold for two matrix functions $s(Y)$ and $s^{\prime}(Y)$, one can show that $s^{\prime}(Y)=s(Y) U(Y)$ with a unitary matrix $U$ (which is not necessarily $Y$ independent).

In appendix A we will show that properties $1 \mathrm{a}-1 \mathrm{c}$ are equivalent to the following two properties:

Property 2a: $S$ satisfies the Collins-Soper equation

$$
\frac{\partial}{\partial Y} S(Y)=\widehat{K} S(Y) \quad \text { for } Y \gg 1 .
$$

$\widehat{K}$ is not necessarily Hermitian, and its relation with $K$ is specified in (A.12). Both (3.25) and (3.26) are meant to be approximations for $Y \gg 1$. We can define a matrix $\widehat{S}(Y)$ that approximates $S(Y)$ for $Y \gg 1$ whilst being an exact solution of the differential equation (3.26) at all $Y$. This equation is then solved by

$$
\widehat{S}(Y)=e^{Y \widehat{K}} \widehat{S}(0) .
$$


Property 2b: There is a value $Y_{1}$ for which $\widehat{S}\left(Y_{1}\right)$ is positive definite.

We show in appendix A that $\widehat{S}(Y)$ is then in fact positive definite for all $Y$. This guarantees that the matrix $S(Y)$ at large $Y$ has an inverse, which according to (3.1) is needed in the cross section formula.

Evidence for the properties just discussed comes from perturbation theory, which can be used to compute the soft factor when the distances $\boldsymbol{z}_{1}, \boldsymbol{z}_{2}$ and $\boldsymbol{y}$ are all small.

- Properties $2 \mathrm{a}$ and $2 \mathrm{~b}$ are easily checked at one-loop order from our explicit calculations in all colour channels, which are reported in section 7 . In this case one finds $\widehat{S}(0)=\mathbb{1}$ and, taking $U=\mathbb{1}$ one has $K=\widehat{K}$ according to (A.12).

- Given property $2 \mathrm{a}$, one can motivate property $2 \mathrm{~b}$ using the perturbative expansions $S(Y)=\mathbb{1}+\mathcal{O}\left(\alpha_{s}\right)$ and $\widehat{K}=\mathcal{O}\left(\alpha_{s}\right)$. One may worry that the expansion of $S(Y)$ has poor convergence, because higher orders in $\alpha_{s}$ can come with higher powers of the large rapidity $Y$. However, according to (3.27) we have $\widehat{S}(0)=\exp (-Y \widehat{K}) S(Y)$ for sufficiently large $Y$. If the perturbative expansions of $S(Y)$ and $\exp (-Y \widehat{K})$ are valid at least in a formal sense, we get an expansion $\widehat{S}(0)=\mathbb{1}+\mathcal{O}\left(\alpha_{s}\right)$ that is free of any large parameter. The eigenvalues of $\widehat{S}(0)$ then have a perturbative expansion around 1, which supports property $2 \mathrm{~b}$ with $Y_{1}=0$, at least for sufficiently small $\alpha_{s}$.

- $S_{q q}$ and $S_{q \bar{q}}$ have been calculated at two-loop order in [74]. The validity of properties 1a and 1c can be explicitly verified from the results in sections 4.2 and 4.4 of that work. This requires a translation between the rapidity regulator used there and the one used here, which we discuss in appendix B. We note that [74] defines $S=s^{\dagger} s$, whilst we choose the order $S=s s^{\dagger}$ in (3.25).

Property $1 \mathrm{~b}$ can then be motivated by the perturbative expansion of $s(Y)$, arguing along the same lines as in the previous point.

- An all-order derivation of the rapidity evolution equation of the DPS soft factor has recently been given in [75]. Our equation (3.26) can be obtained from equation (5.17) in the arXiv version 2 of [75] using the relation (B.1) between the regulator variables $\delta^{+}, \delta^{-}$and $Y_{L}, Y_{R}$. However, more work is needed to establish whether the derivation in [75] holds if one uses either of the associated rapidity regulators.

A few more comments are in order.

- While $S(0)=\mathbb{1}$ by construction (the Wilson line pairs at equal positions give unit matrices, $W^{\dagger} W=\mathbb{1}$ ), one generally has $\widehat{S}(0) \neq \mathbb{1}$. This implies that the evolution equation (3.26) holds for $Y \gg 1$ but not when $Y$ becomes small. We see this already at one loop: as follows from sections 3.3.1 and 3.2.2 of [27], one has $S-\mathbb{1} \propto Y \tanh (Y)$ for the one-loop soft factor, whilst (3.26) truncated to $\mathcal{O}\left(\alpha_{s}\right)$ gives $S-\mathbb{1} \propto Y$.

- For the soft factor needed in collinear factorisation, i.e. for $\boldsymbol{z}_{1}=\boldsymbol{z}_{2}=\mathbf{0}$, we will see in section 4.4 that $S(Y)$ is diagonal in the basis of colour representations for all $Y$. As a consequence, $\widehat{K}$ is diagonal as well. In appendix A we show that one can then 
also choose $s(Y)$ and $K$ to be diagonal. For the soft factor in collinear factorisation, all matrix multiplications in colour space thus become trivial.

- The matrix $s$ replaces the square roots of soft factors in the construction of single parton TMDs by Collins [65], as we will show in section 3.5.

\subsection{Definition of DPDs}

According to (3.1), the DPS cross section involves a product of soft matrices, which using (3.25) can be rewritten as

$$
S^{-1}\left(v_{L}, v_{R}\right) S\left(v_{L}, v_{R}\right) S^{-1}\left(v_{L}, v_{R}\right)=s^{\dagger-1}\left(Y_{R}-Y_{C}\right) s^{-1}\left(Y_{C}-Y_{L}\right),
$$

where $Y_{C}$ is a central rapidity, $Y_{L} \ll Y_{C} \ll Y_{R}$. Restoring parton labels, we then define DPDs by

$$
\begin{aligned}
& F_{a_{1} a_{2}}\left(Y_{C}\right)=\lim _{Y_{L} \rightarrow-\infty} s_{a_{1} a_{2}}^{-1}\left(Y_{C}-Y_{L}\right) F_{\mathrm{us}, a_{1} a_{2}}\left(Y_{L}\right), \\
& F_{b_{1} b_{2}}\left(Y_{C}\right)=\lim _{Y_{R} \rightarrow \infty} s_{b_{1} b_{2}}^{-1}\left(Y_{R}-Y_{C}\right) F_{\mathrm{us}, b_{1} b_{2}}\left(Y_{R}\right)
\end{aligned}
$$

for the distributions in a right-moving and a left-moving proton, respectively. An analogous definition in the $\delta$ regulator scheme was put forward in [74].

From the construction in appendix A it follows that the symmetry relations (3.14) for $S_{a_{1} a_{2}}$ imply corresponding relations

$$
s_{q q}=s_{\bar{q} \bar{q}}^{*}, \quad s_{q \bar{q}}=s_{\bar{q} q}^{*}, \quad s_{q g}=s_{\bar{q} g}^{*}, \quad s_{g g}=s_{g g}^{*}
$$

if the matrices $U_{a_{1} a_{2}}$ in (A.11) are chosen such that they satisfy the relations (A.14). In the expression (3.1) for double Drell-Yan production we can thus rewrite

$$
F_{\mathrm{us}, \bar{q} \bar{q}}^{T}\left(Y_{R}\right) s_{q q}^{\dagger-1}\left(Y_{R}-Y_{C}\right) s_{q q}^{-1}\left(Y_{C}-Y_{L}\right) F_{\mathrm{us}, q q}\left(Y_{L}\right)=F_{\bar{q} \bar{q}}^{T}\left(Y_{C}\right) F_{q q}\left(Y_{C}\right),
$$

where we have omitted the hard-scattering factors $H_{i, q \bar{q}}$ for brevity. A corresponding argument leads to the combination $F_{\bar{q} q}^{T}\left(Y_{C}\right) F_{q \bar{q}}\left(Y_{C}\right)$ in the same process. The production of one Higgs and one electroweak gauge boson involves a term

$$
F_{\mathrm{us}, \bar{q} g}^{T}\left(Y_{R}\right) s_{q g}^{\dagger-1}\left(Y_{R}-Y_{C}\right) s_{q g}^{-1}\left(Y_{C}-Y_{L}\right) F_{\mathrm{us}, q g}\left(Y_{L}\right)=F_{\bar{q} g}^{T}\left(Y_{C}\right) F_{q g}\left(Y_{C}\right)
$$

with mixed quark-gluon and antiquark-gluon DPDs, and likewise one obtains the product $F_{g g}^{T}\left(Y_{C}\right) F_{g g}\left(Y_{C}\right)$ in double Higgs boson production.

In (3.29) we can take the limit of infinite Wilson line rapidities $Y_{L}$ and $Y_{R}$ in the unsubtracted matrix elements. This means that the Wilson lines in the parton operators $\mathcal{O}_{a}$ are along lightlike paths, which leads to important simplifications as discussed in chapter 10.11 of [65]. Let us see that this limit is well behaved in (3.29). The rapidities $Y_{L}$ and $Y_{R}$ in the cross section formula originate from Grammer-Yennie approximations (see e.g. [29]), and one finds that their precise values in (3.31) and (3.32) do not matter as long as $\left|Y_{L}\right|,\left|Y_{R}\right| \gg$ 1. One can thus take the limit of infinite $Y_{L}$ and $Y_{R}$ in these expressions, and thus also 
in the individual factors in (3.29). By contrast, taking infinite Wilson line rapidities in individual unsubtracted DPDs or in the soft factor would lead to rapidity divergences.

The dependence of the DPDs on the central rapidity $Y_{C}$ is easily obtained from (3.24), which gives

$$
\frac{\partial}{\partial Y} s^{-1}(Y)=-K s^{-1}(Y)
$$

and thus

$$
\frac{\partial}{\partial Y_{C}} F\left(Y_{C}\right)=-K F\left(Y_{C}\right)
$$

for DPDs in a right-moving proton, whilst for a left-moving proton one has the opposite sign on the r.h.s. of (3.34).

The preceding construction can be performed for DTMDs and DPDFs alike. Important differences between the two types of distributions arise at the level of ultraviolet renormalisation. In the next subsection, we discuss this for the case of DTMDs, postponing the case of DPDFs to section 5.2.

\subsection{Renormalisation of DTMDs}

The ultraviolet (UV) renormalisation of TMDs arises from vertex and self energy corrections associated with composite operators that contain fields at the same transverse position. Interactions between fields at different transverse positions do not give rise to UV divergences: the finite spacelike distance acts as an effective UV cutoff in the corresponding graphs. Operators that require renormalisation are therefore $[W(\xi) q(\xi)]_{i}$, $\left[W(\xi) G^{+i}(\xi)\right]_{a}$ and their Hermitian conjugates in the collinear matrix elements, as well as $\left[W\left(\xi, v_{L}\right) W^{\dagger}\left(\xi, v_{R}\right)\right]_{i j},\left[W\left(\xi, v_{L}\right) W^{\dagger}\left(\xi, v_{R}\right)\right]_{a b}$ and their Hermitian conjugates in the soft factor. Due to colour SU(3) invariance, the corresponding renormalisation factors are all colour independent. We could in principle choose a different renormalisation scale for each of the four parton operators in $F_{\text {us }}$ and for each of the four corresponding Wilson line products in the soft factor. We choose a slightly simpler variant, taking common renormalisation scales $\mu_{1}$ and $\mu_{2}$ for the operators associated with partons carrying momentum fraction $x_{1}$ and $x_{2}$, respectively. Denoting bare quantities with a subscript $B$, we then have $O_{a}(x, \boldsymbol{y}, \boldsymbol{z})=Z_{\mathrm{us}, a} O_{B, a}(x, \boldsymbol{y}, \boldsymbol{z})$, where $Z_{\mathrm{us}, a}$ is the product of renormalisation factors for the composite operators at transverse positions $\boldsymbol{y}-\frac{1}{2} \boldsymbol{z}$ and $\boldsymbol{y}+\frac{1}{2} \boldsymbol{z}$. Likewise, for the operator defined in (3.16) we have $O_{S, a}(\boldsymbol{y}, \boldsymbol{z})=Z_{S, a} O_{B S, a}(\boldsymbol{y}, \boldsymbol{z})$, where $Z_{S, a}$ is the product of renormalisation factors for the Wilson line pairs at equal transverse positions. ${ }^{2}$ Both $Z_{\mathrm{us}, a}$ and $Z_{S, a}$ are independent of spin and colour, but they differ for quarks and gluons (being equal for quarks and antiquarks due to charge conjugation invariance). They depend on a scale $\mu$ and on Wilson line rapidities, and $Z_{\mathrm{us}, a}$ also depends on the plus-momentum $x p^{+}$ of the relevant parton. We thus have

$$
\begin{aligned}
F_{\mathrm{us}, a_{1} a_{2}}\left(x_{i} ; \mu_{i}, Y_{L}\right) & =Z_{\mathrm{us}, a_{1}}\left(\mu_{1}, Y_{L}, x_{1} p^{+}\right) Z_{\mathrm{us}, a_{2}}\left(\mu_{2}, Y_{L}, x_{2} p^{+}\right) F_{B, \mathrm{us}, a_{1} a_{2}}\left(x_{i} ; Y_{L}\right) \\
S_{a_{1} a_{2}}\left(\mu_{i}, Y\right) & =Z_{S, a_{1}}\left(\mu_{1}, Y\right) Z_{S, a_{2}}\left(\mu_{2}, Y\right) S_{B, a_{1} a_{2}}(Y)
\end{aligned}
$$

\footnotetext{
${ }^{2}$ Our convention for renormalisation factors $Z$ of composite operators corresponds to the one in [65, 76]. Other authors, such as the ones of [77], use $Z^{-1}$ instead.
} 
with $Y=Y_{R}-Y_{L}$. Throughout this work we use the convention that a function with arguments $x_{i}\left(\mu_{i}\right)$ depends on both $x_{1}$ and $x_{2}\left(\mu_{1}\right.$ and $\left.\mu_{2}\right)$. In this and the next subsection, we consider a right-moving proton for definiteness, and we omit transverse position arguments for brevity.

We now derive the renormalisation properties of the matrix $s_{a_{1} a_{2}}(Y)$. Assuming that the $Y$ dependence of $S_{B}(Y)$ is given by (3.26) with a bare kernel $\widehat{K}_{B}$ and defining

$$
\Lambda_{a}(Y)=\frac{\partial}{\partial Y} \log Z_{S, a}(Y)
$$

we readily get

$$
\frac{\partial}{\partial Y} S_{a_{1} a_{2}}(Y)=\widehat{K}_{a_{1} a_{2}}(Y) S_{a_{1} a_{2}}(Y)
$$

with

$$
\widehat{K}_{a_{1} a_{2}}(Y)=\Lambda_{a_{1}}(Y) \mathbb{1}+\Lambda_{a_{2}}(Y) \mathbb{1}+\widehat{K}_{B, a_{1} a_{2}},
$$

where we have dropped the dependence on the renormalisation scales for brevity. The renormalised soft factor hence satisfies (3.26) if $\Lambda_{a}$ is independent of $Y$. This is easily shown for the MS scheme. Expanding $Z_{S}$ in the renormalised coupling, we have $Z_{S}=$ $1+\sum_{n=1}^{\infty} \alpha_{s}^{n} Z_{S}^{(n)}(\epsilon)$ with coefficients $Z_{S}^{(n)}$ being a finite sum of poles in $\epsilon$ for each $n$. The corresponding coefficients in $\Lambda=\sum_{n=1}^{\infty} \alpha_{s}^{n} \Lambda^{(n)}(\epsilon)$ are hence pure pole terms as well. Since $S$ is finite for $\epsilon=0$, the same holds for $\widehat{K}$ according to (3.37), so that the $\epsilon$ poles of $\Lambda$ must cancel those of $\widehat{K}_{B}$ in (3.38). Since the latter are $Y$ independent, the same holds for $\Lambda$. In the $\overline{\mathrm{MS}}$ scheme one can repeat the previous argument after rescaling $\alpha_{s}$ in the expansion of $Z_{S}$ by a factor $S_{\epsilon}$ (see section 7.3.3 for further explanation).

With $\Lambda_{a}$ being $Y$ independent, the solution of (3.36) reads $Z_{S, a}(Y)=Z_{S, a}(0) e^{Y \Lambda_{a}}$. Using (A.11) and (A.12), one finds that

$$
\begin{aligned}
K_{a_{1} a_{2}}\left(\mu_{i}\right) & =\Lambda_{a_{1}}\left(\mu_{1}\right) \mathbb{1}+\Lambda_{a_{2}}\left(\mu_{2}\right) \mathbb{1}+K_{B, a_{1} a_{2}}, \\
s_{a_{1} a_{2}}\left(\mu_{i}, Y\right) & =Z_{s, a_{1}}\left(\mu_{1}, Y\right) Z_{s, a_{2}}\left(\mu_{2}, Y\right) s_{B, a_{1} a_{2}}(Y)
\end{aligned}
$$

with $Z_{s, a}(Y)=\sqrt{Z_{S, a}(0)} e^{Y \Lambda_{a}}$, where we have restored the $\mu$ dependence of the factors. We thus find that the renormalisation of $s(Y)$ is multiplicative and independent of the colour channel, just as the one of $S(Y)$. With bare and renormalised DTMDs defined as in (3.29), we then have

$$
F_{a_{1} a_{2}}\left(x_{i} ; \mu_{i}, Y_{C}\right)=Z_{F, a_{1}}\left(\mu_{1}, Y_{C}, x_{1} p^{+}\right) Z_{F, a_{2}}\left(\mu_{2}, Y_{C}, x_{2} p^{+}\right) F_{B, a_{1} a_{2}}\left(x_{i} ; Y_{C}\right)
$$

with

$$
Z_{F, a}\left(\mu, Y_{C}, x p^{+}\right)=\lim _{Y_{L} \rightarrow-\infty} Z_{s, a}^{-1}\left(\mu, Y_{C}-Y_{L}\right) Z_{\mathrm{us}, a}\left(\mu, Y_{L}, x p^{+}\right)
$$

for a right-moving proton, and correspondingly for a left-moving one. Note that, as explained in chapter 10.11 of [65], one should first take the limit of infinite $Y_{L}$ in the product (3.41) and in $F_{B}\left(Y_{C}\right)$, and then let $\epsilon$ go to zero on the r.h.s. of (3.40). 
We can now derive the $\mu$ dependence of the DTMDs and of their Collins-Soper kernel. Defining

$$
\gamma_{F, a}\left(\mu, Y_{C}, x p^{+}\right)=\frac{\partial}{\partial \log \mu} \log Z_{F, a}\left(\mu, Y_{C}, x p^{+}\right), \quad \gamma_{K, a}(\mu)=-\frac{\partial}{\partial \log \mu} \Lambda_{a}(\mu),
$$

we obtain for a right-moving proton

$$
\begin{aligned}
\frac{\partial}{\partial \log \mu_{1}} F_{a_{1} a_{2}}\left(x_{i} ; \mu_{i}, Y_{C}\right) & =\gamma_{F, a_{1}}\left(\mu_{1}, Y_{C}, x_{1} p^{+}\right) F_{a_{1} a_{2}}\left(x_{i} ; \mu_{i}, Y_{C},\right), \\
\frac{\partial}{\partial \log \mu_{1}} K_{a_{1} a_{2}}\left(\mu_{i}\right) & =-\gamma_{K, a_{1}}\left(\mu_{1}\right) \mathbb{1},
\end{aligned}
$$

and analogous relations for the $\mu_{2}$ dependence, as well as the additional condition

$$
\frac{\partial}{\partial Y_{C}} \gamma_{F, a}\left(\mu, Y_{C}, x p^{+}\right)=\gamma_{K, a}(\mu)
$$

We note that $Z_{F, a}$ and hence $\gamma_{F, a}$ can depend on $Y_{C}$ and $x p^{+}$only via the boost invariant combination $x p^{+} e^{-Y_{C}}$. This will be used to introduce the rapidity parameter $\zeta$ later on (see $(3.50)$ and (5.8)).

It is easy to repeat the above arguments with four different renormalisation scales for the four parton legs of $F$. One then obtains analogues of (3.43) for each scale, with $\gamma_{K, a}$ replaced by $\gamma_{K, a} / 2$ and $\gamma_{F, a}$ by $\gamma_{E, a}$ or $\left(\gamma_{E, a}\right)^{*}$, where $\gamma_{E, a}+\left(\gamma_{E, a}\right)^{*}=\gamma_{F, a}$. The anomalous dimension $\gamma_{E, a}$ has an imaginary part and is for a parton momentum leaving $F$, whereas $\left(\gamma_{E, a}\right)^{*}$ is for a parton momentum entering $F$. This can be seen in the study of the quark form factor [68] (see section 10.12.2 of [65] for an explicit calculation at leading order).

\subsection{Definition of single parton TMDs}

It is instructive to revisit the definition of single parton TMDs in the framework we have just laid out. The colour structure is considerably simplified in this case. The operator $O_{a}(x, \mathbf{0}, \boldsymbol{z})$ in the matrix element for an unsubtracted TMD in (3.11) must carry colour singlet quantum numbers, which is achieved by contracting the operators in (3.9) with $\delta_{j j^{\prime}}$ or $\delta_{a a^{\prime}}$. Likewise, the soft factor for single hard scattering involves the colour singlet projection of the operator $O_{S, a}(\mathbf{0}, \boldsymbol{z})$ defined in (3.16). One can easily adapt the arguments in the previous subsections to this case: the matrices $S, \widehat{S}, s, K$ and $\widehat{K}$ then become single real valued functions. One finds $K=\widehat{K}$, whilst the definition (A.11) simplifies to

$$
s(Y)=e^{Y K} \widehat{S}^{1 / 2}(0),
$$

which is positive and satisfies (3.24). This gives

$$
S(Y)=\widehat{S}(Y)=e^{Y K} \widehat{S}(0)=s\left(Y-Y_{0}\right) s\left(Y_{0}\right)
$$

for $Y \gg 1$ and arbitrary $Y_{0}$. The ambiguity in the choice of $s$, due to unitary transformations in the matrix case, is no longer present. Combining the two previous equations, we deduce that

$$
s(Y)=\sqrt{S(2 Y)} .
$$


The square-root factor in the construction of single parton TMDs by Collins, given in equation (13.106) of [65], can be rewritten as

$$
\sqrt{\frac{S\left(Y_{R}-Y_{C}\right)}{S\left(Y_{R}-Y_{L}\right) S\left(Y_{C}-Y_{L}\right)}}=s^{-1}\left(Y_{C}-Y_{L}\right)
$$

using (3.46) and (3.47). The final TMD is then given by

$$
f_{a}\left(Y_{C}\right)=\lim _{Y_{L} \rightarrow-\infty} s_{a}^{-1}\left(Y_{C}-Y_{L}\right) f_{\mathrm{us}, a}\left(Y_{L}\right),
$$

where we have restored the parton label $a$ to denote quarks, antiquarks and gluons. This form closely resembles the definition in [60,78]; we will expand on this further in appendix B. A related discussion has been given in [79], but the separation into different factors made there differs from ours.

The renormalisation of single parton TMDs is done with the same factors $Z_{\mathrm{us}, a}, Z_{S, a}$ and $Z_{F, a}$ as in section 3.4, and hence involves the same anomalous dimensions. Coming back to our remark after (3.44) and defining

$$
\zeta=2\left(x p^{+}\right)^{2} e^{-2 Y_{C}}
$$

for a right-moving proton, we recover the familiar evolution equations given in (2.3) to (2.8).

\section{Colour}

An essential element in the description of DPS is the colour structure, which is much more involved than in single hard scattering. To deal efficiently with matrices in colour space, we introduce projection operators and make use of the fact that certain colour indices must couple to an overall colour singlet. We derive the combination (4.24) of colour projected quantities that enters the DPS cross section in section 4.2 and establish a number of symmetry relations in section 4.3 . In sections 4.4 and 4.5 we consider the case of collinear factorisation. Projector identities such as the one in figure 2 lead to dramatic simplifications in this case, with soft matrices that are diagonal and depend on only three independent functions as specified in (4.47) to (4.49). These simplifications also allow us to derive the structure (4.53) of the DPS cross section for produced particles carrying colour. A reader who is not interested in technical details may skip the following derivations and will find the principal results in the equations just mentioned.

\subsection{Projection operators}

For the fundamental representation of the group $\mathrm{SU}(N)$, we introduce the operators

$$
\begin{aligned}
& P_{1}^{i i^{\prime} j j^{\prime}}=\frac{1}{N} \delta_{i i^{\prime}} \delta_{j j^{\prime}}, \\
& P_{8}^{i i^{\prime} j j^{\prime}}=2 t_{i i^{\prime}}^{a} t_{j j^{\prime}}^{a},
\end{aligned}
$$


which project the index pairs $i i^{\prime}$ and $j j^{\prime}$ onto a colour singlet or a colour octet, respectively. ${ }^{3}$ In these and all following projection operators, the four indices are coupled to an overall colour singlet. We take $T_{F}=1 / 2$ throughout this paper and do not distinguish between upper and lower colour indices. We will make repeated use of the colour Fierz identity

$$
2 t_{i i^{\prime}}^{a} t_{j j^{\prime}}^{a}=\delta_{i j^{\prime}} \delta_{i^{\prime} j}-\frac{1}{N} \delta_{i i^{\prime}} \delta_{j j^{\prime}}
$$

For the adjoint representation we use [32, 80, 81]

$$
\begin{aligned}
P_{1}^{a a^{\prime} b b^{\prime}} & =\frac{1}{N^{2}-1} \delta^{a a^{\prime}} \delta^{b b^{\prime}}, \\
P_{A}^{a a^{\prime} b b^{\prime}} & =\frac{1}{N} f^{a a^{\prime} c} f^{b b^{\prime} c}, \\
P_{S}^{a a^{\prime} b b^{\prime}} & =\frac{N}{N^{2}-4} d^{a a^{\prime} c} d^{b b^{\prime} c}, \\
P_{D}^{a a^{\prime} b b^{\prime}} & =\frac{1}{2}\left(\delta^{a b} \delta^{a^{\prime} b^{\prime}}-\delta^{a b^{\prime}} \delta^{a^{\prime} b}\right)-P_{A}^{a a^{\prime} b b^{\prime}}, \\
P_{27}^{a a^{\prime} b b^{\prime}} & =\frac{1}{2}\left(\delta^{a b} \delta^{a^{\prime} b^{\prime}}+\delta^{a b^{\prime}} \delta^{a^{\prime} b}\right)-P_{S}^{a a^{\prime} b b^{\prime}}-P_{1}^{a a^{\prime} b b^{\prime}} .
\end{aligned}
$$

The subscript on $P$ always denotes the colour representation onto which index pairs $a a^{\prime}$ and $b b^{\prime}$ are projected, with $A$ denoting the antisymmetric and $S$ the symmetric octet. ${ }^{4}$ Useful relations for calculating with the $f$ and $d$ tensors are given in [83].

The operator $P_{D}=P_{10}+P_{\overline{10}}$ projects on the sum of the decuplet and antidecuplet representations. For any tensor $M^{a a^{\prime} b b^{\prime}}$ that transforms as an overall colour singlet, the projections on decuplet and antidecuplet are equal (and can hence be added without loss of information). Following appendix A of [34], one can show this by first decomposing $M^{a a^{\prime} b b^{\prime}}$ on $P_{R}^{a b a^{\prime} b^{\prime}}$ with $R=1, A, S, 10, \overline{10}, 27$ and then projecting on $P_{R}^{a a^{\prime}} b b^{\prime}$. This result can be traced back to the fact that $\left(P_{10}\right)^{a a^{\prime} b b^{\prime}}-(P \overline{10})^{a a^{\prime} b b^{\prime}}$ is odd but all $P_{R}^{a b a^{\prime} b^{\prime}}$ are even under the simultaneous exchange $\left(a \leftrightarrow b, a^{\prime} \leftrightarrow b^{\prime}\right)$.

We will further need projectors for mixed fundamental and adjoint indices:

$$
\begin{aligned}
& P_{1}^{i i^{\prime} a a^{\prime}}=P_{1}^{a a^{\prime} i i^{\prime}}=\frac{1}{\sqrt{N\left(N^{2}-1\right)}} \delta_{i i^{\prime}} \delta^{a a^{\prime}}, \\
& P_{A}^{i i^{\prime} a a^{\prime}}=P_{A}^{a a^{\prime} i i^{\prime}}=\sqrt{\frac{2}{N} t_{i i^{\prime}}^{c} f^{a a^{\prime} c},} \\
& P_{S}^{i i^{\prime} a a^{\prime}}=P_{S}^{a a^{\prime} i i^{\prime}}=\sqrt{\frac{2 N}{N^{2}-4}} t_{i i^{\prime}}^{c} d^{a a^{\prime} c} .
\end{aligned}
$$

The normalisation factors are chosen such as to yield the simple symmetry and projection properties to be discussed next.

\footnotetext{
${ }^{3}$ We use the term "octet" to denote the adjoint representation, which of course has dimension 8 only for $\mathrm{SU}(3)$.

${ }^{4}$ For $N>3$ a further representation appears when decomposing the product of two octets (see table 9.4 in [82]). This representation is sometimes labelled as $R=0$, since it has dimension 0 for $N=3$. The projector $P_{27}$ in (4.3) corresponds to $P_{27}+P_{0}$ in the case of general $N$ [81].
} 
To ease our notation, we introduce double indices $\underline{i}=\left(i i^{\prime}\right), \underline{a}=\left(a a^{\prime}\right)$. Here and in the following, $\underline{i}, \underline{j}, \underline{k}$ are in the fundamental representation, $\underline{a}, \underline{b}, \underline{c}$ are in the adjoint, whereas $\underline{r}, \underline{s}, \underline{t}, \underline{u}, \underline{v}, \underline{w}$ can belong to either representation. Repeated double indices are summed over, so that for adjoint indices we have

$$
M_{1}^{\underline{\underline{r}} \underline{a}} M_{2}^{\underline{a} \underline{s}}=M_{1}^{\underline{r} a a^{\prime}} M_{2}^{a a^{\prime}} \underline{\underline{s}} .
$$

Some care is required for indices of the fundamental representation. In fundamental Wilson lines $W$ and in projection operators $P_{R}$, the first index always transforms as a triplet and the second index as an antitriplet. For such cases, we define matrix multiplication with a transposition

$$
M_{1}^{\underline{r} \underline{i}} M_{2}^{\underline{i} \underline{s}}=M_{1}^{\underline{r} i i^{\prime}} M_{2}^{i i^{\prime} \underline{\underline{s}}} .
$$

In soft matrices $S$ and in the matrix elements giving DPDs, the order of triplet and antitriplet indices depends on the parton channel $(q$ or $\bar{q})$ and in $S$ also on the direction of the Wilson lines (along $v_{L}$ or $v_{R}$ ). Contraction of double indices should always be done such that a triplet index is contracted with an antitriplet one. This ensures proper behaviour of the result under gauge transformations.

With these definitions, the projection operators have the symmetry

$$
P_{R}^{\underline{r} \underline{s}}=P_{R}^{\underline{s}} \underline{\underline{r}}
$$

and the projection property

$$
P_{\bar{R}}^{\underline{r} \underline{s}} P_{R^{\prime}}^{\underline{s}}=\delta_{R R^{\prime}} P_{\bar{R}}^{\underline{\underline{r}} \underline{t}}
$$

for any pair of projectors $P_{R}, P_{R^{\prime}}$ with a common double index $\underline{s}$ in either the fundamental or the adjoint representation. In the octet sector, it is understood that $\delta_{8 A}=\delta_{A 8}=\delta_{8 S}=$ $\delta_{S 8}=1$ but $\delta_{A S}=\delta_{S A}=0$ in (4.8). The normalisation is given by

$$
P_{\bar{R}}^{\underline{r}} \underline{s} P_{R^{\prime}}^{\underline{r} \underline{s}}=\delta_{R R^{\prime}} m(R),
$$

where

$$
m(R)=P_{\bar{R}}^{\underline{r}} \underline{r}
$$

is the multiplicity of the representation, i.e. $m(1)=1, m(8)=m(A)=m(S)=8$, $m(D)=20$ and $m(27)=27$ for $\mathrm{SU}(3)$. Since the projectors above form a basis of the space of rank-four tensors $M$ that transform as an $\mathrm{SU}(3)$ singlet, any such tensor $M$ can be decomposed as

$$
M^{\underline{r} \underline{s}}=\sum_{R} \frac{1}{m(R)} P_{\underline{R}}^{\underline{r}} \underline{\underline{s}}\left(P_{\underline{\underline{t}} \underline{\underline{u}}}^{\underline{\underline{t}}} M^{\underline{\underline{u}}}\right),
$$

where the sum is over all representations $R$ given in (4.1), (4.3) and (4.4), as applicable. The contraction of two colour singlet tensors is then given by

$$
M_{1}^{\underline{r} \underline{s}} M_{2}^{\underline{r} \underline{s}}=\sum_{R} \frac{1}{m(R)}\left(P_{R}^{\underline{r}} \underline{s} M_{1}^{\underline{r}} \underline{\underline{s}}\right)\left(P_{\bar{R}}^{\underline{t} \underline{u}} M_{2}^{\underline{t} \underline{u}}\right) .
$$

For representation labels $R$, we will not use the summation convention, i.e. summation over $R$ will always be indicated explicitly. 


\subsection{Colour structure of the DPS cross section}

The projection operators just introduced allow us to rewrite the DPS cross section in a compact way. We start by defining DPDs that are projected on a definite colour representation $R$. Using the general decomposition in (4.11), we can write

$$
F_{a_{1}}^{\underline{r}_{1} \underline{r}_{2}}=\sum_{R} \frac{1}{\varepsilon_{a_{1}}(R) \varepsilon_{a_{2}}(R)} \frac{1}{\mathcal{N}_{a_{1}} \mathcal{N}_{a_{2}}} \frac{1}{\sqrt{m(R)}}{ }^{R} F_{a_{1} a_{2}} P_{R}^{\underline{\underline{r}}_{1} \underline{\underline{r}}_{2}}
$$

with

$$
{ }^{R} F_{a_{1} a_{2}}=\varepsilon_{a_{1}}(R) \varepsilon_{a_{2}}(R) \mathcal{N}_{a_{1}} \mathcal{N}_{a_{2}} \frac{1}{\sqrt{m(R)}} P_{R}^{\underline{s}_{1}} \underline{s}_{2} F_{a_{1} a_{2}}^{\underline{s}_{1}} \underline{s}_{2}
$$

Note that the lower indices $a_{1}, a_{2}$ denote the parton species and polarisation, not colour. The factors

$$
\varepsilon_{a}(R)= \begin{cases}i & \text { if } a \text { is a gluon and } R=A \\ 1 & \text { otherwise }\end{cases}
$$

ensure that the collinear distributions ${ }^{A} F_{q g}\left(x_{i}, \boldsymbol{y}\right)$ and ${ }^{A} F_{\bar{q} g}\left(x_{i}, \boldsymbol{y}\right)$ and their polarised counterparts are real valued (rather than imaginary), as are the collinear distributions in all other channels. This is shown in the next subsection. The prefactors $\mathcal{N}_{a}$ are given by

$$
\mathcal{N}_{q}=\mathcal{N}_{\bar{q}}=\sqrt{N}, \quad \mathcal{N}_{g}=\sqrt{N^{2}-1}
$$

and likewise for polarised partons. They ensure that colour singlet distributions ${ }^{1} F_{a_{1} a_{2}}$ involve a sum over the colours of the two partons. The definitions here coincide with the ones in $[27,34]$, with the notational change

$$
\left.{ }^{D} F_{g g}\right|_{\text {here }}=\left.{ }^{10+\overline{10}} F_{g g}\right|_{\text {ref. [34] }}=\left.\frac{1}{\sqrt{2}}\left({ }^{10} F_{g g}+{ }^{\overline{10}} F_{g g}\right)\right|_{\text {ref. [27] }}
$$

and its analogues for polarised gluons. For the discussion of renormalisation and the shortdistance expansion, it is useful to project the partonic operators (3.10) on definite colour representations as well. Introducing

$$
{ }^{R} O_{a}^{\underline{r}}=\varepsilon_{a}(R) \mathcal{N}_{a} P_{\bar{R}}^{\underline{r s}} O_{a}^{\underline{s}}
$$

and defining unsubtracted DPDs ${ }^{R} F_{\mathrm{us}, a_{1} a_{2}}$ in analogy to (4.14), we obtain

$$
2 \pi \delta\left(p^{+}-p^{+}\right) 2 p^{+R} F_{\mathrm{us}, a_{1} a_{2}}=\frac{1}{\sqrt{m(R)}}\left\langle\left. p^{\prime}\right|^{R} O_{a_{1}}^{R}{ }^{R}{ }_{\frac{r}{a_{2}}} \mid p\right\rangle
$$

from (3.11) and the projection property (4.8).

The soft factor for DPS producing colour singlet particles in the hard interactions carries two times four indices. Its projection on different representations is defined by

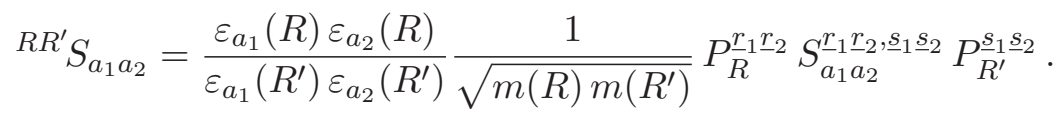

$R$ specifies the colour representation for the Wilson lines with positive rapidity, and $R^{\prime}$ the one for the Wilson lines with negative rapidity. Notice that the $\varepsilon$ factors cancel for 
$R=R^{\prime}$. In analogy to (4.20) we define projections $R R^{\prime} s$ and ${ }^{R} R^{\prime} K$ of the matrix $s$ and of the Collins-Soper kernel $K$, as well as projections $R R^{\prime}\left(S^{-1}\right)$ and $R R^{\prime}\left(s^{-1}\right)$ of the inverse matrices $S^{-1}$ and $s^{-1}$ in colour space. Using the same argument as in (4.12), one can show that

$$
\sum_{R^{\prime}} R R^{\prime}\left(S_{a_{1} a_{2}}^{-1}\right) R^{\prime} R^{\prime \prime} S_{a_{1} a_{2}}=\sum_{R^{\prime}} R R^{\prime}\left(s_{a_{1} a_{2}}^{-1}\right)^{R^{\prime} R^{\prime \prime}} s_{a_{1} a_{2}}=\delta_{R R^{\prime \prime}}
$$

We see that matrix multiplication in the space of four colour indices $\left(\underline{r}_{1} \underline{r}_{2}\right)=\left(r_{1} r_{1}^{\prime} r_{2} r_{2}^{\prime}\right)$ turns into matrix multiplication in the space of colour representations $R$. The matrix decomposition (3.25) can thus be rewritten as

$$
{ }^{R R^{\prime \prime}} S_{a_{1} a_{2}}(Y)=\sum_{R^{\prime}} R R^{\prime} s_{a_{1} a_{2}}\left(Y-Y_{0}\right)^{R^{\prime} R^{\prime \prime}}\left(s_{a_{1} a_{2}}^{\dagger}\left(Y_{0}\right)\right),
$$

and the definition (3.29) of DPDs in a right-moving proton as

$$
{ }^{R} F_{a_{1} a_{2}}\left(Y_{C}\right)=\lim _{Y_{L} \rightarrow-\infty} \sum_{R^{\prime}} R R^{\prime}\left(s_{a_{1} a_{2}}^{-1}\left(Y_{C}-Y_{L}\right)\right){ }^{R^{\prime}} F_{\mathrm{us}, a_{1} a_{2}}\left(Y_{L}\right) .
$$

According to (3.1) and (3.31), the cross section for the production of colour neutral particles involves the product

$$
X=H_{a_{1} b_{1}} H_{a_{2} b_{2}} F_{b_{1} b_{2}}^{\underline{r}_{1} \underline{r}_{2}} F_{a_{1} a_{2}}^{\underline{r}_{1} \underline{r}_{2}}=\frac{H_{a_{1} b_{1}}}{\mathcal{N}_{a_{1}} \mathcal{N}_{b_{1}}} \frac{H_{a_{2} b_{2}}}{\mathcal{N}_{a_{2}} \mathcal{N}_{b_{2}}} \sum_{R} \eta_{a_{1} a_{2}}(R)^{R} F_{b_{1} b_{2}}{ }^{R} F_{a_{1} a_{2}} .
$$

Here we have combined the factors $\varepsilon_{a_{1}}(R) \varepsilon_{b_{1}}(R) \varepsilon_{a_{2}}(R) \varepsilon_{b_{2}}(R)$ into

$$
\eta_{a_{1} a_{2}}(R)=\frac{1}{\varepsilon_{a_{1}}^{2}(R) \varepsilon_{a_{2}}^{2}(R)},
$$

using that the only channels that produce colourless particles are $q \bar{q}$ and $g g$ annihilation. Multiplied with a flux factor, the combinations $H_{a b} /\left(\mathcal{N}_{a} \mathcal{N}_{b}\right)$ turn into the hard-scattering cross sections in the final factorisation formula. The factor $\mathcal{N}_{a} \mathcal{N}_{b}$ is $N$ for $q \bar{q}$ annihilation and $N^{2}-1$ for $g g$ annihilation and ensures that one takes an average over the colour states of the two colliding partons, as is appropriate for a parton-level cross section.

\subsection{Symmetry properties}

As already remarked, multiplication of matrices and vectors, $S$ and $F$, in the space of colour indices $\left(\underline{r}_{1} \underline{r}_{2}\right)$ turns into multiplication of matrices and vectors in the space of representations $R$. For brevity, we refer to the former as "colour space" and to the latter as "representation space". Note that our representation space only corresponds to the part of colour space in which the indices $\left(\underline{r}_{1} \underline{r}_{2}\right)$ are coupled to an overall colour singlet. We now derive some important properties of $S$ and of the related matrices $s$ and $K$ in representation space.

In (3.20) we have seen that the matrix $S_{a_{1} a_{2}}$ is Hermitian in colour space,

$$
S_{a_{1} a_{2}}^{\underline{r}_{1}} \underline{\underline{r}}_{2} \underline{s}_{1} \underline{s}_{2}=\left(S_{a_{1} a_{2}}^{\underline{s}_{1}} \underline{\underline{s}}_{2} \underline{r}_{1} \underline{\underline{r}}_{2}\right)^{*} .
$$

Under Hermitian conjugation, an index pair $\underline{i}=\left(i, i^{\prime}\right)$ in $S$ changes its transformation properties: if $i$ transforms as an $\mathrm{SU}(3)$ triplet in $S$, it transforms as an antitriplet in $S^{\dagger}$ 
and vice versa. The colour projectors satisfy $P_{R}^{i i^{\prime} j j^{\prime}}=\left(P_{R}^{i \prime i} j^{\prime} j\right)^{*}, P_{R}^{i i^{\prime} a a^{\prime}}=\left(P_{R}^{i^{\prime} i a a^{\prime}}\right)^{*}$ and $P_{R}^{a a^{\prime} b b^{\prime}}=\left(P_{R}^{a a^{\prime} b b^{\prime}}\right)^{*}$, so that the rule for contraction with the colour projectors stated below (4.6) is satisfied if $S$ is contracted with $P_{R}$ and $S^{\dagger}$ with $P_{R}^{*}$. We thus find that

$$
R R^{\prime} S_{a_{1} a_{2}}=\left({ }^{\prime} R S_{a_{1} a_{2}}\right)^{*}
$$

i.e. $S$ is also Hermitian in representation space. In analogy, the fact that the Collins-Soper kernel $K_{a_{1} a_{2}}$ is Hermitian in colour space implies that it is Hermitian in representation space as well.

We now turn to the relations between soft factors for quarks and antiquarks. The relations (3.14) imply that

$$
R R^{\prime} S_{a_{1} a_{2}}=\frac{\eta_{a_{1} a_{2}}\left(R^{\prime}\right)}{\eta_{a_{1} a_{2}}(R)}\left(R R^{\prime} S_{\bar{a}_{1} \bar{a}_{2}}\right)^{*}=\frac{\eta_{a_{1} a_{2}}\left(R^{\prime}\right)}{\eta_{a_{1} a_{2}}(R)} R^{\prime} R S_{\bar{a}_{1} \bar{a}_{2}}
$$

where $\bar{a}$ denotes the antiparton of $a$ (with the convention $\bar{g}=g$ ). The complex conjugation of projection operators implicit in the first step corresponds to the fact that an index $i$ transforming as a triplet in $S_{a_{1} a_{2}}$ transforms as an antitriplet in $S_{\bar{a}_{1} \bar{a}_{2}}$ and vice versa. Complex conjugation of the $\varepsilon$ factors in the definition (4.20) gives the factors $\eta$ in (4.28). In the second step we have simply used the property (4.27). We now recall that the relations (3.14) for $S$ translate into analogous relations (3.30) for $s$. By taking the rapidity derivative, we find corresponding relations for $K$ as well. In analogy to (4.28) we can thus derive

$$
R R^{\prime} K_{a_{1} a_{2}}=\frac{\eta_{a_{1} a_{2}}\left(R^{\prime}\right)}{\eta_{a_{1} a_{2}}(R)} R^{\prime} R K_{\bar{a}_{1} \bar{a}_{2}}
$$

We will use this in section 5.1 when making rapidity evolution explicit in the DPS cross section. A different way to connect soft factors for quarks and antiquarks is to note that the operator $O_{S, q}$ in (3.16) is related with its analogue $O_{S, \bar{q}}$ for antiquarks by

$$
\left[O_{S, q}\left(\boldsymbol{y}, \boldsymbol{z} ; v_{L}, v_{R}\right)\right]^{i i^{\prime}, l l^{\prime}}=\left[O_{S, \bar{q}}\left(\boldsymbol{y},-\boldsymbol{z} ; v_{L}, v_{R}\right)\right]^{i^{\prime}, l^{\prime} l}
$$

Multiplying with colour projectors according to the rule stated below (4.6), one obtains

$$
\begin{aligned}
{ }^{R R^{\prime}} S_{q q}\left(\boldsymbol{z}_{1}, \boldsymbol{z}_{2}, \boldsymbol{y}\right) & ={ }^{R R^{\prime}} S_{q \bar{q}}\left(\boldsymbol{z}_{1},-\boldsymbol{z}_{2}, \boldsymbol{y}\right) \\
& ={ }^{R R^{\prime}} S_{\bar{q} q}\left(-\boldsymbol{z}_{1}, \boldsymbol{z}_{2}, \boldsymbol{y}\right)={ }^{R}{ }^{\prime} S_{\bar{q} \bar{q}}\left(-\boldsymbol{z}_{1},-\boldsymbol{z}_{2}, \boldsymbol{y}\right),
\end{aligned}
$$

where the arguments $v_{L}, v_{R}$ are the same in all four expressions. Relations analogous to (4.31) hold for ${ }^{R R^{\prime}} K_{a_{1} a_{2}}$, provided that ${ }^{R R^{\prime}} U_{a_{1} a_{2}}$ satisfies the requirements (A.15).

Let us finally investigate the consequences of charge conjugation invariance. Charge conjugation transforms $t^{a} A^{\mu a}(\xi) \rightarrow-\left(t^{a}\right)^{*} A^{\mu a}(\xi)$, which can be derived from the familiar transformation properties of quark fields and charge conjugation invariance of the interaction term $-g \bar{q} t^{a} A^{\mu a} \gamma_{\mu} q$ in the QCD Lagrangian. As a result, a fundamental Wilson line transforms as

$$
W_{i j} \rightarrow W_{i j}^{*}
$$


For soft factors ${ }^{R R^{\prime}} S$ involving gluons, we rewrite all adjoint Wilson lines as [84]

$$
W^{a b}=2 \operatorname{tr}\left(t^{a} W t^{b} W^{\dagger}\right),
$$

where the Wilson lines on the r.h.s. are in the fundamental representation, express $f^{a b c}$ and $d^{a b c}$ as traces of products of matrices $t^{a}, t^{b}, t^{c}$ and then eliminate all these matrices by repeated use of the colour Fierz identity (4.2). After these steps, we have for all parton combinations $a_{1}$ and $a_{2}$ a representation of ${ }^{R R^{\prime}} S_{a_{1} a_{2}}$ in terms of traces of fundamental Wilson lines $W$ and $W^{\dagger}$, where thanks to the $\epsilon$ factors in the definition (4.20) no explicit factors of $i$ appear. The transformation (4.32) then readily implies that ${ }^{R} R^{\prime} S_{a_{1} a_{2}}$ is real valued, and with (4.27) we furthermore obtain that it is symmetric in representation space, i.e. we have ${ }^{R} R^{\prime} S={ }^{\prime} R S$, where for brevity we omit the subscripts $a_{1} a_{2}$.

To show that $R R_{S}^{\prime}$ and ${ }^{R} R^{\prime} K$ are also real valued, we translate the derivation starting in (A.7) into representation space, projecting all relevant matrices as specified in (4.20). It is then easy to see that $R R^{\prime} \widehat{S}$ and $R R^{\prime} \widehat{K}$ are real valued. To establish that $R R^{\prime}\left(\widehat{S}^{1 / 2}\right)$ is real valued, we note that $R R^{\prime} \widehat{S}$ has positive eigenvalues, which is readily seen by projecting the eigenvalue equation $\widehat{S} v=\lambda v$ from colour space to representation space and recalling that in colour space $\widehat{S}$ is a positive matrix. According to the discussion below (A.10), the square root of a positive real matrix is real. Choosing the matrix $U$ in (A.11) and (A.12) such that ${ }^{R R^{\prime}} U$ is real, we find that both ${ }^{R R^{\prime}} s$ and ${ }^{R R^{\prime}} K$ are real. Since ${ }^{R R^{\prime}} K$ is Hermitian, it is also symmetric: ${ }^{R} R^{\prime} K={ }^{\prime} R K$.

Combining the symmetry relations (4.28) and (4.31) with the fact that ${ }^{R R^{\prime}} S$ is symmetric in $R$ and $R^{\prime}$, we obtain

$$
{ }^{R R^{\prime}} S_{q q}\left(\boldsymbol{z}_{1}, \boldsymbol{z}_{2}, \boldsymbol{y}\right)={ }^{R R^{\prime}} S_{q q}\left(-\boldsymbol{z}_{1},-\boldsymbol{z}_{2}, \boldsymbol{y}\right)
$$

An analogous relation holds for ${ }^{R R^{\prime}} K_{q q}$.

The quark and gluon operators in (3.3) and (3.6) satisfy $\left[\mathcal{O}_{a}^{r r^{\prime}}\right]^{\dagger}(y, z)=\mathcal{O}_{a}^{r^{\prime} r}(y,-z)$, where the colour index $r$ pertains to the fields at $y+\frac{1}{2} z$ and $r^{\prime}$ to the fields at $y-\frac{1}{2} z$. For colour projected unsubtracted DPDFs this yields the relation

$$
\left[{ }^{R} F_{\mathrm{us}, a_{1} a_{2}}\left(x_{i}, \boldsymbol{y}\right)\right]^{*}={ }^{R} F_{\mathrm{us}, a_{1} a_{2}}\left(x_{i}, \boldsymbol{y}\right) .
$$

Let us explicitly show this for ${ }^{A} F_{\mathrm{us}, q g}$. Using that $\varepsilon_{q}(A) \varepsilon_{g}(A)=i$ and $\left(P_{A}^{j j^{\prime} a a^{\prime}}\right)^{*}=-P_{A}^{j^{\prime} j a} a^{\prime a}$, we have

$$
\begin{aligned}
& {\left[{ }^{A} F_{\mathrm{us}, q g}\left(x_{i}, \boldsymbol{y}\right)\right]^{*}} \\
& \quad=c \int d z_{1}^{-} d z_{2}^{-} d y^{-}\left[i P_{A}^{j j^{\prime} a a^{\prime}} e^{i\left(x_{1} z_{1}^{-}+x_{2} z_{2}^{-}\right) p^{+}}\left\langle p\left|\mathcal{O}_{q}^{j j^{\prime}}\left(y, z_{1}\right) \mathcal{O}_{g}^{a a^{\prime}}\left(0, z_{2}\right)\right| p\right\rangle\right]^{*} \\
& \quad=c \int d z_{1}^{-} d z_{2}^{-} d y^{-} i P_{A}^{j^{\prime} j a^{\prime} a} e^{-i\left(x_{1} z_{1}^{-}+x_{2} z_{2}^{-}\right) p^{+}}\left\langle p\left|\mathcal{O}_{q}^{j^{\prime} j}\left(y,-z_{1}\right) \mathcal{O}_{g}^{a^{\prime} a}\left(0,-z_{2}\right)\right| p\right\rangle \\
& \quad={ }^{A} F_{\mathrm{us}, q g}\left(x_{i}, \boldsymbol{y}\right)
\end{aligned}
$$

where in the last step we used that $\boldsymbol{z}_{1}=\boldsymbol{z}_{2}=\mathbf{0}$. Here $c=c^{*}$ is a product of kinematic and numerical factors not essential for the argument. We see that the factor $\varepsilon_{g}(A)=i$ 


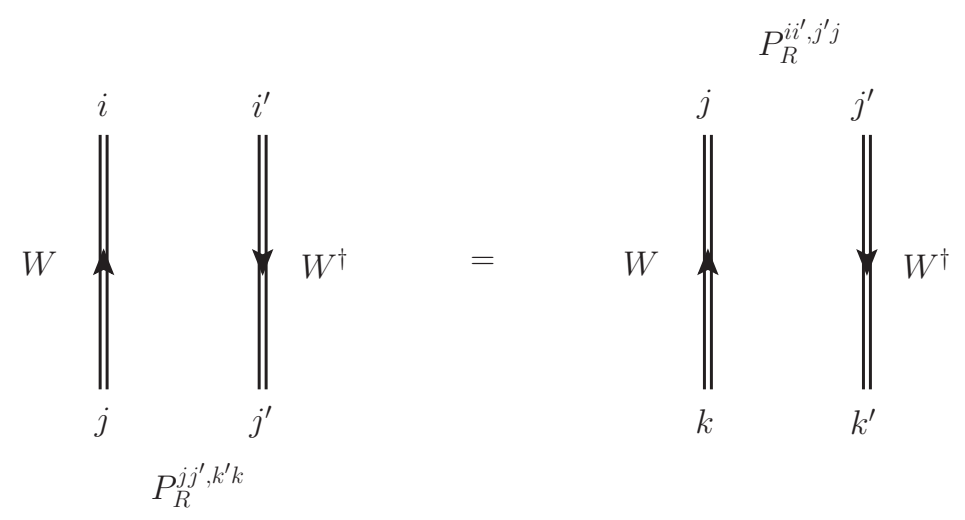

Figure 2. Graphical representation of the relation (4.38). The Wilson lines are understood to be interacting, i.e. any number of gluons may be attached to them in a Feynman graph.

compensates the behaviour of $P_{A}^{j j^{\prime} a a^{\prime}}$ under complex conjugation. One easily checks that $\left[\varepsilon_{a_{1}}(R) \varepsilon_{a_{2}}(R) P_{R}^{r r^{\prime} s s^{\prime}}\right]^{*}=\varepsilon_{a_{1}}(R) \varepsilon_{a_{2}}(R) P_{R}^{r^{\prime} r} s^{\prime} s$ for all projectors, which ensures the general validity of (4.35). Given that $R R_{S}^{\prime}$ is real valued, we finally find that

$$
\left[{ }^{R} F_{a_{1} a_{2}}\left(x_{i}, \boldsymbol{y}\right)\right]^{*}={ }^{R} F_{a_{1} a_{2}}\left(x_{i}, \boldsymbol{y}\right) .
$$

In all colour channels and for all parton combinations, DPDFs are thus real valued.

\subsection{Simplification of soft factors in collinear factorisation}

In the soft factors relevant for collinear factorisation, one should set $\boldsymbol{z}_{1}=\boldsymbol{z}_{2}=\mathbf{0}$ in the definitions (3.12) and (3.15). Corresponding Wilson lines in the scattering amplitude and its complex conjugate are then at the same transverse position. This simplifies the colour structure significantly, as we will now demonstrate. In the remainder of this subsection, it is understood that all soft factors are taken at $\boldsymbol{z}_{1}=\boldsymbol{z}_{2}=\mathbf{0}$.

The projection operators on Wilson lines obey the relation

$$
W_{i j} P_{R}^{j j^{\prime}, k^{\prime} k} W_{j^{\prime} i^{\prime}}^{\dagger}=W_{j k} P_{R}^{i i^{\prime}, j^{\prime} j} W_{k^{\prime} j^{\prime}}^{\dagger}
$$

for $R=1,8$, where it is understood that the Wilson lines $W$ and $W^{\dagger}$ are in the same direction and at the same position. For $R=1$ this is trivial, and for $R=8$ it readily follows from the colour Fierz identity (4.2). A graphical representation of (4.38) is shown in figure 2. Remarkably, an analogous relation holds also for adjoint Wilson lines

$$
W_{a b} P_{R}^{b b^{\prime}, c c^{\prime}} W_{b^{\prime} a^{\prime}}^{\dagger}=W_{b c} P_{R}^{a a^{\prime}, b b^{\prime}} W_{c^{\prime} b^{\prime}}^{\dagger}
$$

and for the mixed case

$$
W_{a b} P_{R}^{b b^{\prime}, k^{\prime} k} W_{b^{\prime} a^{\prime}}^{\dagger}=W_{j k} P_{R}^{a a^{\prime}, j^{\prime} j} W_{k^{\prime} j^{\prime}}^{\dagger}
$$

in all relevant representations $R$. This can be shown by following the steps in and after (4.33). For the extended soft factor, the relations (4.38), (4.39) and (4.40) imply

$$
P_{R}^{\underline{s} \underline{t}}\left(S_{a_{1} a_{2}}\right) \cdots \underline{\underline{r}} \cdots=P_{R}^{\underline{r}} \underline{\underline{s}}\left(S_{a_{1} a_{2}}\right) \cdots \underline{\underline{s}} \cdots,
$$


where we have displayed only one of the four double index pairs $\left(\underline{r}_{i}, \underline{s}_{i}\right)$ in $\left(S_{a_{1} a_{2}}\right) \underline{\underline{r}}_{1} \underline{\underline{r}}_{2}, \underline{\underline{s}}_{2}, \underline{\underline{s}}_{3} \underline{\underline{r}}_{4}$ for better readability, the other three pairs remaining untouched. Instead of making a colour projection for the fields at $\xi^{+}=\xi^{-}=0$, one can thus make the same projection for the fields at infinity. This yields an important relation between a specific projection of the extended soft factor in (3.12) and the basic one in (3.15). With (4.41) and the projection property (4.8) we have

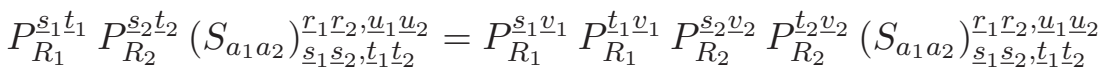

$$
\begin{aligned}
& =P_{R_{1}}^{\underline{r}_{1}} \underline{\underline{s}}_{1} P_{R_{1}}^{\underline{u}_{1}} \underline{\underline{t}}_{1} P_{R_{2}}^{\underline{r}_{2} \underline{s}_{2}} P_{R_{2}}^{\underline{u}_{2} \underline{t}_{2}}\left(S_{a_{1} a_{2}}\right) \underline{\underline{s}}_{1} \underline{\underline{s}}_{1} \underline{\underline{s}}_{2}, \underline{\underline{v}}_{1} \underline{\underline{t}}_{1} \underline{\underline{t}}_{2},
\end{aligned}
$$

where $\underline{r}, \underline{s}, \ldots, \underline{v}$ can each be in the fundamental or adjoint representation. Projecting this on the remaining open indices, we obtain

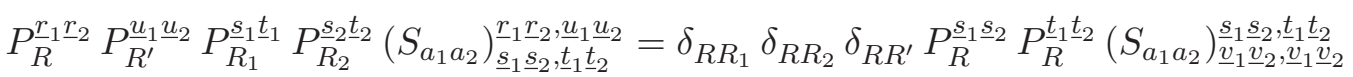

$$
\begin{aligned}
& =\delta_{R R_{1}} \delta_{R R_{2}} \delta_{R R^{\prime}} m(R)^{R R} S_{a_{1} a_{2}},
\end{aligned}
$$

where in the last step we have used the definition (4.20). We furthermore have

$$
\begin{aligned}
& R R^{\prime} S_{a_{1} a_{2}} \frac{\varepsilon_{a_{1}}\left(R^{\prime}\right) \varepsilon_{a_{2}}\left(R^{\prime}\right)}{\varepsilon_{a_{1}}(R) \varepsilon_{a_{2}}(R)} \sqrt{m(R) m\left(R^{\prime}\right)}=P_{R}^{\underline{r}_{1}} \underline{\underline{r}}_{2} P_{R^{\prime}}^{\underline{t}_{1}} \underline{\underline{t}}_{2}\left(S_{a_{1} a_{2}}\right) \underline{\underline{r}}_{\underline{s}_{1}} \underline{\underline{s}}_{2}, \underline{\underline{s}}_{2} \underline{\underline{s}}_{1} \underline{\underline{t}}_{2} \underline{\underline{s}}_{2} \\
& =P_{R}^{\underline{r}_{2} \underline{s}_{2}} P_{R^{\prime}}^{\underline{t}_{2}} \underline{\underline{s}}_{2}\left(S_{a_{1} a_{2}}\right)_{\underline{s}_{1}}^{\underline{r}_{1}} \underline{\underline{r}}_{2}, \underline{\underline{r}}_{1} \underline{\underline{s}}_{1} \underline{t}_{2}
\end{aligned}
$$

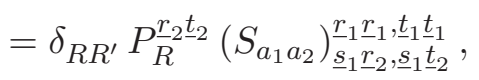

which shows that at $\boldsymbol{z}_{1}=\boldsymbol{z}_{2}=\mathbf{0}$ the soft matrix is diagonal in the colour representations $R$ and $R^{\prime}$. Note that this is not the case for nonzero $\boldsymbol{z}_{1}, \boldsymbol{z}_{2}$, as follows immediately from the explicit one-loop expressions in section 7.2.

The soft factors discussed so far correspond to the same parton species entering a hard scattering process in the amplitude and its conjugate. As already mentioned at the end of section 3.1, there is also interference between $q$ and $\bar{q}$, between $q$ and $g$, and between $\bar{q}$ and $g$. The corresponding Wilson line products in the soft factor are then $W_{i j} W_{i^{\prime} j^{\prime}}, W_{i j} W_{a^{\prime} b^{\prime}}$, etc. The relevant projection operators for coupling colour indices in these cases are given in equations (9) and (11) of [32]. Relations analogous to (4.38) can be easily derived for these cases, where again the colour projections for the gluon fields at $\xi^{+}=\xi^{-}=0$ in the Wilson lines can be traded for the colour projections of the fields at infinity. From this, relations analogous to (4.43) and (4.44) can be derived for the soft factors associated with interference between parton species in the hard scattering.

We now relate soft factors in the adjoint representation with those in the fundamental one. For the mixed quark-gluon soft factor we have

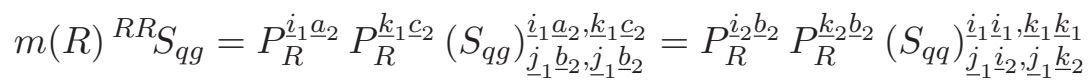

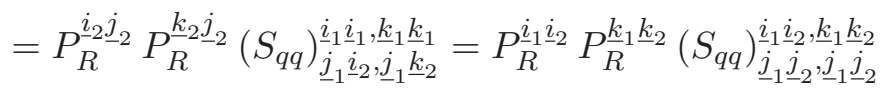

$$
\begin{aligned}
& =m(R){ }^{R R} S_{q q}
\end{aligned}
$$


if $R$ is in the singlet or octet channels, where in the second and the forth step we have used (4.41) for the indices associated with parton $a_{2}$ in $S_{a_{1} a_{2}}$. Writing

$$
\begin{aligned}
m(R)^{R R} S_{g g} & =P_{R}^{\underline{a}_{1} \underline{a}_{2}} P_{R}^{\underline{c}_{1} \underline{c}_{2}}\left(S_{g g}\right) \underline{\underline{a}}_{1} \underline{\underline{a}}_{1} \underline{\underline{a}}_{2}, \underline{\underline{c}}_{1} \underline{\underline{b}}_{2} \underline{\underline{b}}_{2} \\
& =P_{R}^{\underline{\underline{i}}_{1} \underline{a}_{1}} P_{R}^{\underline{\underline{i}}_{1} \underline{a}_{2}} P_{R}^{\underline{\underline{k}}_{1} \underline{c}_{1}} P_{R}^{\underline{k}_{1} \underline{c}_{2}}\left(S_{g g}\right) \underline{\underline{a}}_{1} \underline{\underline{a}}_{1}, \underline{\underline{b}}_{2}, \underline{\underline{b}}_{1} \underline{\underline{b}}_{1} \underline{\underline{c}}_{2}
\end{aligned}
$$

for $R=1, A, S$ and using (4.41) for all index pairs, one can reduce $S_{g g}$ to $S_{q q}$ in the colour singlet and octet channels as well. The only independent soft matrix elements for collinear DPS factorisation (in channels with the same parton species in the amplitude and its conjugate) are therefore

$$
{ }^{11} S={ }^{11} S_{q q}={ }^{11} S_{q g}={ }^{11} S_{g q}={ }^{11} S_{g g}=1
$$

and

$$
\begin{aligned}
{ }^{88} S & ={ }^{88} S_{q q}={ }^{A A} S_{q g}={ }^{S S} S_{q g}={ }^{A A} S_{g q}={ }^{S S} S_{g q}={ }^{A A} S_{g g}={ }^{S S} S_{g g}, \\
{ }^{D D} S & ={ }^{D D} S_{g g}, \\
{ }^{2727} S & ={ }^{2727} S_{g g},
\end{aligned}
$$

where one can also replace one or both labels $q$ with $\bar{q}$ in the soft factors involving quarks. We recall that each factor in (4.48) depends on $\boldsymbol{y}$, on a rapidity difference, and on two renormalisation scales $\mu_{1}$ and $\mu_{2}$. In the colour singlet channel the soft matrix elements are unity because all Wilson lines are contracted pairwise to $W W^{\dagger}=W^{\dagger} W=\mathbb{1}$.

As noted in [74], there is a remarkable identity

$$
{ }^{88} S(\boldsymbol{y})=S_{g}(\boldsymbol{y}),
$$

where $S_{g}$ is the soft factor for single TMD factorisation with a gluon initiated hard scattering such as $g g \rightarrow H$ [85]. Its definition reads

$$
S_{g}(\boldsymbol{z})=\left\langle 0\left|P_{1}^{\underline{a}} \underline{\underline{b}}\left[O_{S, g}(\mathbf{0}, \boldsymbol{z})\right]^{\underline{a}, \underline{b}}\right| 0\right\rangle,
$$

where $O_{S, g}$ is obtained from (3.16) by replacing the fundamental Wilson lines with adjoint ones. One can easily prove (4.49) by applying the colour Fierz identity (4.2) to ${ }^{88} S$ in the quark representation and to $S_{g}$ after converting adjoint Wilson lines into fundamental ones using (4.33). It is understood that the two renormalisation scales $\mu_{1}, \mu_{2}$ in ${ }^{88} S(\boldsymbol{y})$ must be taken equal in (4.49).

\subsection{Interlude: collinear factorisation for coloured particle production}

In collinear factorisation, we may consider the production of coloured particles, a prominent example for DPS being double dijet production. As pointed out in section 2.1 of [29], the steps for showing factorisation in this case lead to the extended soft factor (3.12). Instead of the product in (3.1), the cross section then involves products of the form

$$
\begin{aligned}
& X=H_{a_{1} b_{1}}^{\underline{s}_{1}} \underline{\underline{t}}_{1} H_{a_{2} b_{2}}^{\underline{s}_{2} \underline{t}_{2}}\left[F_{\mathrm{us}, b_{1} b_{2}}^{T}\left(Y_{R}\right) S^{-1}\left(Y_{R}-Y_{L}\right)\right]^{\underline{r}_{1} \underline{r}_{2}}
\end{aligned}
$$

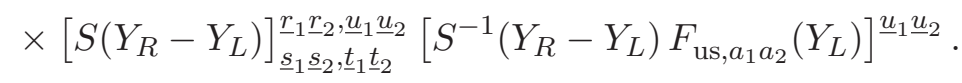




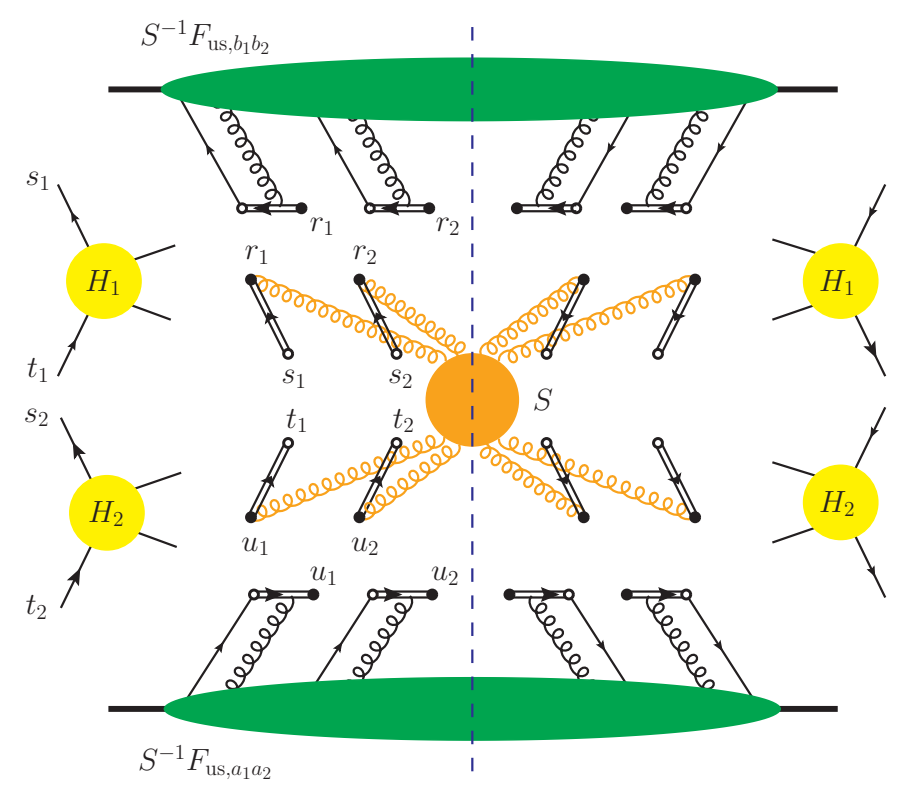

Figure 3. Graphical representation of colour index contractions in the factorised double dijet cross section. The indices in this figure correspond to the ones given in (4.51). The corresponding primed indices in the complex conjugate part of the graph are not shown.

A pictorial representation is given in figure 3. Colour indices $s, t$ in the hard scattering factor $H^{s s^{\prime} t t^{\prime}}$ are for the incoming partons in the amplitude, whilst $s^{\prime}, t^{\prime}$ are for the incoming partons in the conjugate amplitude. These indices are contracted with colour indices of the extended soft factor. The inverse soft factors $S^{-1}$ in (4.51) remove soft gluons from the unsubtracted collinear factors $F_{\text {us }}$ as usual; they are hence the soft factors with left and right moving Wilson lines colour contracted as in (3.15). We have omitted their parton labels, given that after colour projection they are independent of the parton species as shown in the previous subsection. In the extended soft factor, the representation of each Wilson line must match the parton entering in the corresponding hard-scattering subprocess. This includes combinations such as $W_{a b}\left(\boldsymbol{\xi}, v_{L}\right) W_{k l}^{\dagger}\left(\boldsymbol{\xi}, v_{R}\right)$, which corresponds to a right-moving quark scattering on a left-moving gluon. It is not difficult to generalise the relation (4.43) between the extended and usual soft factors to such cases, so that we can omit parton labels in the extended soft factor as well. Inserting projection operators as in (4.12), we can thus rewrite (4.51) as

$$
\begin{aligned}
& X=\sum_{R, R^{\prime}, R_{1}, R_{2}} \frac{P_{R_{1}}^{\underline{s}_{1} \underline{\underline{t}}_{1}} H_{a_{1} \underline{b}_{1}}^{\underline{s}_{1}} P_{R_{2}}^{\underline{s}_{2}} \underline{\underline{t}}_{2} H_{a_{2} \underline{b}_{2}}^{\underline{s}_{2} \underline{\underline{t}}_{2}}}{m(R) m\left(R^{\prime}\right) m\left(R_{1}\right) m\left(R_{2}\right)} P_{R}^{\underline{r}_{1} \underline{r}_{2}}\left[F_{\mathrm{us}, b_{1} b_{2}}^{T}\left(Y_{R}\right) S^{-1}\left(Y_{R}-Y_{L}\right)\right]^{\underline{r}_{1} \underline{r}_{2}} \\
& \times \delta_{R R_{1}} \delta_{R R_{2}} \delta_{R R^{\prime}} m(R)^{R R} S\left(Y_{R}-Y_{L}\right) P_{R^{\prime}}^{\underline{u}_{1}} \underline{u}_{2}\left[S^{-1}\left(Y_{R}-Y_{L}\right) F_{\mathrm{us}, a_{1} a_{2}}\left(Y_{L}\right)\right]^{\underline{u}_{1}} \underline{u}_{2} \\
& =\sum_{R} \frac{P_{R}^{\underline{s}_{1} \underline{t}_{1}} H_{a_{1} b_{1}}^{\underline{\underline{s}}_{1} \underline{\underline{t}}_{1}} P_{R}^{\underline{\underline{s}}_{2} \underline{\underline{t}}_{2}} H_{a_{2} \underline{b}_{2}}^{\underline{s}_{2} \underline{\underline{t}}_{2}}}{m^{3}(R)} P_{R}^{\underline{r}_{1} \underline{r}_{2}} F_{\mathrm{us}, b_{1} b_{2}}^{\underline{r}_{1} \underline{\underline{r}}_{2}}\left(Y_{R}\right)^{R R} S^{-1}\left(Y_{R}-Y_{L}\right) P_{R}^{\underline{u}_{1} \underline{u}_{2}} F_{\mathrm{us}, a_{1} a_{2}}^{\underline{u}_{1} \underline{u}_{2}}\left(Y_{L}\right) \text {, }
\end{aligned}
$$

where in the last step we used that $R^{\prime} S^{-1}$ is diagonal in $R$ and $R^{\prime}$. Splitting ${ }^{R R} S$ as in (4.22) and using that ${ }^{R R} s$ is real valued, we obtain colour projected DTMDs from (4.23) 
and the analogue of (4.14) for $F_{\text {us }}$. This yields the main result of this subsection:

$$
\begin{aligned}
& X=\sum_{R} \frac{1}{\varepsilon_{a_{1}}(R) \varepsilon_{b_{1}}(R) \varepsilon_{a_{2}}(R) \varepsilon_{b_{2}}(R)} \frac{1}{\mathcal{N}_{a_{1}} \mathcal{N}_{b_{1}} \mathcal{N}_{a_{2}} \mathcal{N}_{b_{2}}} \frac{P_{R}^{\underline{s}_{1}} \underline{\underline{t}}_{1} H_{a_{1} b_{1}}^{\underline{s}_{1}} P_{R}^{\underline{t}_{1}} \underline{\underline{\underline{s}}}_{2} \underline{\underline{t}}_{2} H_{a_{2} \underline{b}_{2}}^{\underline{s}_{2} \underline{t}_{2}}}{m^{2}(R)} \\
& \times{ }^{R} F_{\mathrm{us}, b_{1} b_{2}}\left(Y_{R}\right){ }^{R R} s^{-1}\left(Y_{R}-Y_{C}\right){ }^{R R} s^{-1}\left(Y_{C}-Y_{L}\right){ }^{R} F_{\mathrm{us}, a_{1} a_{2}}\left(Y_{L}\right) \\
& =\sum_{R}{ }^{R} H_{a_{1} b_{1}}{ }^{R} H_{a_{2} b_{2}}{ }^{R} F_{b_{1} b_{2}}{ }^{R} F_{a_{1} a_{2}} .
\end{aligned}
$$

The colour structure of the collinear cross section is thus surprisingly simple: we have the same DPDs in (4.53) as in the formula (4.24) for colour singlet production, and the only new ingredients are the colour projected hard-scattering factors ${ }^{5}$

$$
{ }^{R} H_{a b}=\frac{1}{\varepsilon_{a}(R) \varepsilon_{b}(R)} \frac{1}{\mathcal{N}_{a} \mathcal{N}_{b}} \frac{P_{R}^{\underline{s}} \underline{\underline{t}} H_{a b}^{\underline{s}} \underline{\underline{t}}}{m(R)} .
$$

For the production of a colour singlet system, the hard scattering subprocess has the structure

$$
H_{a}^{\underline{s}} \underline{\underline{t}}=\delta_{s t} \delta_{s^{\prime} t^{\prime}} H_{a b}
$$

Using (4.10) we thus find

$$
P_{R}^{\underline{s} \underline{t}} H_{a}^{\underline{s}} \underline{\underline{t}}=m(R) H_{a b},
$$

so that ${ }^{R} H_{a b}$ reproduces $H_{a b} /\left(\mathcal{N}_{a} \mathcal{N}_{b}\right)$ divided by $\varepsilon_{a}(R) \varepsilon_{b}(R)$, which is absorbed into the sign factor $\eta_{a_{1} a_{2}}(R)$ in (4.24).

\section{Factorisation at small transverse momenta}

We are now in a situation to give the TMD factorisation formula for DPS processes. Let us consider the production of two particles or groups of particles with transverse momenta $\boldsymbol{q}_{1}, \boldsymbol{q}_{2}$ and invariant masses $Q_{1}, Q_{2}$. Both $Q_{1}$ and $Q_{2}$ are required to be large, and we denote their generic size by $Q$ for the purpose of power counting. Both transverse momenta, whose generic size we denote by $q_{T}$, are required to be small compared with $Q$. Up to power corrections in $\Lambda / Q$ and $\left|\boldsymbol{q}_{i}\right| / Q$, the DPS cross section with measured transverse momenta reads

$$
\begin{gathered}
\frac{d \sigma_{\mathrm{DPS}}}{d x_{1} d x_{2} d \bar{x}_{1} d \bar{x}_{2} d^{2} \boldsymbol{q}_{1} d^{2} \boldsymbol{q}_{2}}=\frac{1}{C} \sum_{a_{1}, a_{2}, b_{1}, b_{2}} \hat{\sigma}_{a_{1} b_{1}}\left(Q_{1}, \mu_{1}\right) \hat{\sigma}_{a_{2} b_{2}}\left(Q_{2}, \mu_{2}\right) \\
\times \int \frac{d^{2} \boldsymbol{z}_{1}}{(2 \pi)^{2}} \frac{d^{2} \boldsymbol{z}_{2}}{(2 \pi)^{2}} d^{2} \boldsymbol{y} e^{-i \boldsymbol{q}_{1} \boldsymbol{z}_{1}-i \boldsymbol{q}_{2} \boldsymbol{z}_{2}} W_{a_{1} a_{2} b_{1} b_{2}}\left(\bar{x}_{i}, x_{i}, \boldsymbol{z}_{i}, \boldsymbol{y} ; \mu_{i}, \nu\right),
\end{gathered}
$$

with

$$
\begin{aligned}
& W_{a_{1} a_{2} b_{1} b_{2}}\left(\bar{x}_{i}, x_{i}, \boldsymbol{z}_{i}, \boldsymbol{y} ; \mu_{i}, \nu\right)=\Phi\left(\nu \boldsymbol{y}_{+}\right) \Phi\left(\nu \boldsymbol{y}_{-}\right) \\
& \quad \times \sum_{R} \eta_{a_{1} a_{2}}(R)^{R} F_{b_{1} b_{2}}\left(\bar{x}_{i}, \boldsymbol{z}_{i}, \boldsymbol{y} ; \mu_{i}, \bar{\zeta}\right){ }^{R} F_{a_{1} a_{2}}\left(x_{i}, \boldsymbol{z}_{i}, \boldsymbol{y} ; \mu_{i}, \zeta\right)
\end{aligned}
$$

${ }^{5}$ The factors ${ }^{R} H$ in (4.54) differ from the ones in equation (2.11) of [29]. Here we project index pairs in the $t$-channel $\left(s, s^{\prime}\right.$ etc.) onto a definite colour representation $R$, whereas in [29] this was done for index pairs in the $s$-channel $(s, t$ etc.). 
and

$$
\boldsymbol{y}_{ \pm}=\boldsymbol{y} \pm \frac{1}{2}\left(\boldsymbol{z}_{1}-\boldsymbol{z}_{2}\right)
$$

We recall that we write arguments $x_{i}$ if a function depends on both $x_{1}, x_{2}$, and likewise for all other variables. The combinatorial factor $C$ is equal to 2 if the systems produced by the two hard scatters are identical and equal to 1 otherwise. The factor $\eta_{a_{1} a_{2}}(R)$ was defined in (4.25); it is equal to -1 if $R=A$ and exactly one of $a_{1}$ and $a_{2}$ is a gluon and equal to 1 otherwise. The subprocess cross sections are given by

$$
\hat{\sigma}_{a_{1} b_{1}}\left(Q_{1}^{2}, \mu_{1}^{2}\right)=\frac{1}{2 Q_{1}^{2}} \frac{H_{a_{1} b_{1}}\left(Q_{1}^{2}, \mu_{1}^{2}\right)}{\mathcal{N}_{a_{1}} \mathcal{N}_{b_{1}}}
$$

and likewise for index 2 . Here, $1 /\left(2 Q_{1}^{2}\right)$ is the standard flux factor, and $1 /\left(\mathcal{N}_{a_{1}} \mathcal{N}_{b_{1}}\right)$ implements colour averaging over the initial state as noted after (4.24). The sum over $a_{1}, a_{2}, b_{1}, b_{2}$ in (5.1) runs over both parton species and polarisations, following the notation specified in section 3.1. Hard-scattering cross sections and DPDs carry transverse Lorentz indices for transverse quark and linear gluon polarisation, which must be contracted appropriately. For later discussion, it is useful to rewrite the integration measure in (5.1) as

$$
d^{2} \boldsymbol{y} d^{2} \boldsymbol{z}_{1} d^{2} \boldsymbol{z}_{2} e^{-i \boldsymbol{q}_{1} \boldsymbol{z}_{1}-i \boldsymbol{q}_{2} \boldsymbol{z}_{2}}=d^{2} \boldsymbol{Z} d^{2} \boldsymbol{y}_{+} d^{2} \boldsymbol{y}_{-} e^{-i\left(\boldsymbol{q}_{1}+\boldsymbol{q}_{2}\right) \boldsymbol{Z}-i\left(\boldsymbol{q}_{1}-\boldsymbol{q}_{2}\right)\left(\boldsymbol{y}_{+}-\boldsymbol{y}_{-}\right) / 2}
$$

with

$$
\boldsymbol{Z}=\frac{1}{2}\left(\boldsymbol{z}_{1}+\boldsymbol{z}_{2}\right)
$$

The function $\Phi\left(\nu \boldsymbol{y}_{ \pm}\right)$in (5.2) regulates the ultraviolet region. It is intimately related with the cross talk between double and single hard scattering and was introduced in the recent work [30]. This is discussed in section 5.3, where we show how to combine the contributions from DPS, SPS and their interference to obtain the physical cross section. Apart from this function, the factorisation formula (5.1) is identical to the form that emerged from the analysis of lowest-order graphs in section 2 of [27]. This is because our DPD definition in section 3.3 absorbs all relevant effects of soft gluon exchange in the cross section, leading to the crucial relations (3.31) and (3.32).

Of course, the scale and rapidity parameter dependence of the DTMDs in (5.1) does not arise from lowest-order graphs but from the full analysis in section 3.4. In the next section, we give the final forms (5.7) and (5.11) of the corresponding evolution equations, as well as their explicit solutions (5.17) and (5.19) at the level of DPDs and of the cross section, respectively. In section 5.2 we derive the evolution equations (5.22) to (5.25) of DPDFs and give the explicit form (5.27) of their rapidity dependence.

When combining SPS and DPS in the physical cross section, one must take care of interference effects and of double counting. A systematic framework for this has been proposed in [30], and in section 5.3 of the present paper we characterise the individual terms in the master formula (5.30) for the cross section with measured transverse momenta. 


\subsection{DTMD evolution: renormalisation scale and rapidity}

Let us rewrite the evolution equations for the DTMDs using the projection operators introduced in section 4 . The renormalisation group equation (3.43) then reads

$$
\frac{\partial}{\partial \log \mu_{1}}{ }^{R} F_{a_{1} a_{2}}\left(x_{i}, \boldsymbol{z}_{i}, \boldsymbol{y} ; \mu_{i}, \zeta\right)=\gamma_{F, a_{1}}\left(\mu_{1}, x_{1} \zeta / x_{2}\right)^{R} F_{a_{1} a_{2}}\left(x_{i}, \boldsymbol{z}_{i}, \boldsymbol{y} ; \mu_{i}, \zeta\right)
$$

for the scale $\mu_{1}$, and in analogy for $\mu_{2}$. Here we have introduced the rapidity parameter

$$
\zeta=2 x_{1} x_{2}\left(p^{+}\right)^{2} e^{-2 Y_{C}},
$$

where $p$ is the proton momentum. ${ }^{6}$ This extends the definition (3.50) for SPS to the case of DPS in a way that is symmetric in the two momentum fractions $x_{1}, x_{2}$. As discussed after (3.44), the correct rapidity argument of $\gamma_{F, a}$ in (5.7) is $x_{1} \zeta / x_{2}=2\left(x_{1} p^{+}\right)^{2} e^{-2 Y_{C}}$, since the corresponding UV divergent subgraphs depend on the momentum of parton 1 (whose plus-component is $x_{1} p^{+}$), but not on the momentum of parton 2. For the DPD of the left moving proton with momentum $\bar{p}$ we define accordingly

$$
\bar{\zeta}=2 \bar{x}_{1} \bar{x}_{2}\left(\bar{p}^{-}\right)^{2} e^{2 Y_{C}} .
$$

Note that

$$
\zeta \bar{\zeta}=x_{1} \bar{x}_{1} x_{2} \bar{x}_{2}\left(2 p^{+} \bar{p}^{-}\right)^{2}=Q_{1}^{2} Q_{2}^{2} .
$$

Rewriting the rapidity derivative as $\partial / \partial Y_{C}=-2 \partial / \partial \log \zeta=2 \partial / \partial \log \bar{\zeta}$, one obtains from (3.34) the Collins-Soper equation

$$
\frac{\partial}{\partial \log \zeta}{ }^{R} F_{a_{1} a_{2}}\left(x_{i}, \boldsymbol{z}_{i}, \boldsymbol{y} ; \mu_{i}, \zeta\right)=\frac{1}{2} \sum_{R^{\prime}}^{R R^{\prime}} K_{a_{1} a_{2}}\left(\boldsymbol{z}_{i}, \boldsymbol{y} ; \mu_{i}\right){ }^{R^{\prime}} F_{a_{1} a_{2}}\left(x_{i}, \boldsymbol{z}_{i}, \boldsymbol{y} ; \mu_{i}, \zeta\right)
$$

for colour projected DTMDs in a right moving proton. The Collins-Soper equation for a left moving proton has the same form, with $\zeta$ replaced by $\bar{\zeta}$. The scale dependence of the Collins-Soper kernel in (3.43) now reads

$$
\frac{\partial}{\partial \log \mu_{1}} R R^{\prime} K_{a_{1} a_{2}}\left(\boldsymbol{z}_{i}, \boldsymbol{y} ; \mu_{i}\right)=-\gamma_{K, a_{1}}\left(\mu_{1}\right) \delta_{R R^{\prime}}
$$

and correspondingly for $\mu_{2}$. It is thus only the diagonal elements of ${ }^{\prime} K$ that have UV divergences and depend on the renormalisation scales $\mu_{1}$ and $\mu_{2}$. Let us recall that the kernel $K_{a_{1} a_{2}}$ and the anomalous dimensions $\gamma_{F, a}$ and $\gamma_{K, a}$ depend on the colour representation of the parton (quarks or antiquarks vs. gluons) but not on their flavour or polarisation.

It is easy to solve the rapidity and renormalisation scale evolution with general starting scales $\mu_{01}, \mu_{02}$ for $\mu_{1}, \mu_{2}$ and $\zeta_{0}$ for $\zeta$. Renormalisation scale evolution gives

$$
\begin{aligned}
& { }^{R} F_{a_{1} a_{2}}\left(x_{i}, \boldsymbol{z}_{i}, \boldsymbol{y} ; \mu_{i}, \zeta\right)={ }^{R} F_{a_{1} a_{2}}\left(x_{i}, \boldsymbol{z}_{i}, \boldsymbol{y} ; \mu_{0 i}, \zeta\right) \\
& \times \exp \left[\int_{\mu_{01}}^{\mu_{1}} \frac{d \mu}{\mu} \gamma_{F, a_{1}}\left(\mu, x_{1} \zeta / x_{2}\right)+\int_{\mu_{02}}^{\mu_{2}} \frac{d \mu}{\mu} \gamma_{F, a_{2}}\left(\mu, x_{2} \zeta / x_{1}\right)\right] .
\end{aligned}
$$

\footnotetext{
${ }^{6} \mathrm{~A}$ different definition (going back to [86]) was used in [27], namely $\left.x_{1} x_{2} \zeta^{2}\right|_{\text {ref. }[27]}=\left.\zeta\right|_{\text {here }}$.
} 
Using the rapidity evolution in (5.11) we write

$$
{ }^{R} F_{a_{1} a_{2}}\left(x_{i}, \boldsymbol{z}_{i}, \boldsymbol{y} ; \mu_{i}, \zeta\right)=\sum_{R^{\prime}} R R^{\prime} \exp \left[K_{a_{1} a_{2}}\left(\boldsymbol{z}_{i}, \boldsymbol{y} ; \mu_{i}\right) \log \frac{\sqrt{\zeta}}{\sqrt{\zeta_{0}}}\right] R^{\prime} F_{a_{1} a_{2}}\left(x_{i}, \boldsymbol{z}_{i}, \boldsymbol{y} ; \mu_{i}, \zeta_{0}\right),
$$

where $R^{\prime}$ exp is to be understood as a matrix exponential, i.e.

$$
R R^{\prime} \exp (M)=\delta_{R R^{\prime}}+{ }^{R R^{\prime}} M+\sum_{n=2}^{\infty} \sum_{R_{2}, \ldots, R_{n}} \frac{R R_{2} M \ldots R_{n} R^{\prime} M}{n !} .
$$

We now split

$$
R R^{\prime} K_{a_{1} a_{2}}\left(\boldsymbol{z}_{i}, \boldsymbol{y} ; \mu_{i}\right)=\delta_{R R^{\prime}}\left[{ }^{1} K_{a_{1}}\left(\boldsymbol{z}_{1} ; \mu_{1}\right)+{ }^{1} K_{a_{2}}\left(\boldsymbol{z}_{2} ; \mu_{2}\right)\right]+{ }^{R R^{\prime}} M_{a_{1} a_{2}}\left(\boldsymbol{z}_{i}, \boldsymbol{y}\right),
$$

where ${ }^{1} K_{a}(\boldsymbol{z} ; \mu)$ is the kernel in the Collins-Soper equation (2.7) for a single parton TMD. The colour singlet label 1 on $K$ is given for consistency with our later notation in section 6.2.2. By virtue of (2.8) and (5.12), the matrix ${ }^{R R^{\prime}} M$ is independent of $\mu_{1}$ and $\mu_{2}$. Combining (5.13) and (5.14) with (5.16), we obtain the relation between the DTMD at initial and final scales in the form

$$
\begin{aligned}
& { }^{R} F_{a_{1} a_{2}}\left(x_{i}, \boldsymbol{z}_{i}, \boldsymbol{y} ; \mu_{i}, \zeta\right) \\
& =\exp \left\{\int_{\mu_{01}}^{\mu_{1}} \frac{d \mu}{\mu}\left[\gamma_{a_{1}}(\mu)-\gamma_{K, a_{1}}(\mu) \log \frac{\sqrt{x_{1} \zeta / x_{2}}}{\mu}\right]+{ }^{1} K_{a_{1}}\left(\boldsymbol{z}_{1} ; \mu_{01}\right) \log \frac{\sqrt{\zeta}}{\sqrt{\zeta_{0}}}\right. \\
& \left.\quad+\int_{\mu_{02}}^{\mu_{2}} \frac{d \mu}{\mu}\left[\gamma_{a_{2}}(\mu)-\gamma_{K, a_{2}}(\mu) \log \frac{\sqrt{x_{2} \zeta / x_{1}}}{\mu}\right]+{ }^{1} K_{a_{2}}\left(\boldsymbol{z}_{2} ; \mu_{02}\right) \log \frac{\sqrt{\zeta}}{\sqrt{\zeta_{0}}}\right\} \\
& \quad \times \sum_{R^{\prime}} R R^{\prime} \exp \left[M_{a_{1} a_{2}}\left(\boldsymbol{z}_{i}, \boldsymbol{y}\right) \log \frac{\sqrt{\zeta}}{\sqrt{\zeta_{0}}}\right]{ }^{\prime} F_{a_{1} a_{2}}\left(x_{i}, \boldsymbol{z}_{i}, \boldsymbol{y} ; \mu_{01}, \mu_{02}, \zeta_{0}\right),
\end{aligned}
$$

where we have used the explicit form (2.5) of $\gamma_{F, a}$. The exponential in the second and third lines is just the generalisation to two partons of the evolution factor for a single parton TMD, as is readily seen by solving the system of equations in (2.3) to (2.8). It resums both double and single logarithms. The last line in (5.17) describes the mixing between different colour representations $R$ under rapidity evolution and involves a single logarithm. The double logarithms in the evolution of ${ }^{R} F_{a_{1} a_{2}}\left(x_{i}, \boldsymbol{z}_{i}, \boldsymbol{y} ; \mu_{i}, \zeta\right)$ are thus the same as those for a product of two single TMDs with appropriate arguments.

The symmetry relation (4.29) for ${ }^{R R^{\prime}} K$ translates into an analogous relation for ${ }^{R} R^{\prime} M$. Using that $b_{i}$ is the antiparton of $a_{i}$ in the cross section formula (5.1) and that $M_{a_{1} a_{2}}$ does not depend on parton spins, we can thus rewrite

$$
\eta_{a_{1} a_{2}}(R) R^{R R^{\prime}} \exp \left[M_{b_{1} b_{2}} \log \frac{\sqrt{\bar{\zeta}}}{\sqrt{\zeta_{0}}}\right]{ }^{R^{\prime}} F_{b_{1} b_{2}}=\eta_{a_{1} a_{2}}\left(R^{\prime}\right){ }^{R^{\prime}} F_{b_{1} b_{2}} R^{\prime} R \exp \left[M_{a_{1} a_{2}} \log \frac{\sqrt{\bar{\zeta}}}{\sqrt{\zeta_{0}}}\right]
$$

when inserting the evolved form of ${ }^{R} F_{b_{1} b_{1}}$ into the expression (5.2) of $W$. This allows us to combine the matrix exponentials associated with the two DPDs. Further using that 
one has equal anomalous dimensions $\gamma_{a_{i}}=\gamma_{b_{i}}$ and $\gamma_{K, a_{i}}=\gamma_{K, b_{i}}$ and equal Collins-Soper kernels ${ }^{1} K_{a_{i}}={ }^{1} K_{b_{i}}$, we obtain

$$
\begin{aligned}
W_{a_{1} a_{2} b_{1} b_{2}}= & \exp \left\{\int_{\mu_{01}}^{\mu_{1}} \frac{d \mu}{\mu}\left[\gamma_{a_{1}}(\mu)-\gamma_{K, a_{1}}(\mu) \log \frac{Q_{1}^{2}}{\mu^{2}}\right]+{ }^{1} K_{a_{1}}\left(\boldsymbol{z}_{1} ; \mu_{01}\right) \log \frac{Q_{1} Q_{2}}{\zeta_{0}}\right. \\
& \left.+\int_{\mu_{02}}^{\mu_{2}} \frac{d \mu}{\mu}\left[\gamma_{a_{2}}(\mu)-\gamma_{K, a_{2}}(\mu) \log \frac{Q_{2}^{2}}{\mu^{2}}\right]+{ }^{1} K_{a_{2}}\left(\boldsymbol{z}_{2} ; \mu_{02}\right) \log \frac{Q_{1} Q_{2}}{\zeta_{0}}\right\} \\
\times & \Phi\left(\nu \boldsymbol{y}_{+}\right) \Phi\left(\nu \boldsymbol{y}_{-}\right) \sum_{R R^{\prime}} \eta_{a_{1} a_{2}}(R){ }^{R} F_{b_{1} b_{2}}\left(\bar{x}_{i}, \boldsymbol{z}_{i}, \boldsymbol{y} ; \mu_{01}, \mu_{02}, \zeta_{0}\right) \\
& \times{ }^{R R^{\prime}} \exp \left[M_{a_{1} a_{2}}\left(\boldsymbol{z}_{i}, \boldsymbol{y}\right) \log \frac{Q_{1} Q_{2}}{\zeta_{0}}\right] R^{\prime} F_{a_{1} a_{2}}\left(x_{i}, \boldsymbol{z}_{i}, \boldsymbol{y} ; \mu_{01}, \mu_{02}, \zeta_{0}\right) .
\end{aligned}
$$

We have used the relation (5.10) for combining the arguments of the logarithms and expressing them in terms of the physical scales $Q_{1}$ and $Q_{2}$. The variables $\zeta$ and $\bar{\zeta}$ have thus completely disappeared from the physical cross section. This is not the case for the factorisation scales $\mu_{i}$ in (5.19) and in the hard scattering cross sections $\hat{\sigma}$. As is well known, the dependence on these scales only cancels up to un-calculated higher orders in $\alpha_{s}$.

\subsection{DPDF evolution}

We now turn to DPDFs, which appear not only in the factorisation formula for DPS with integrated transverse momenta, but also in the short-distance expansion of DTMDs, as we will see in section 6.2. We have derived in section 4.4 that the soft factor ${ }^{R} R^{\prime} S_{a_{1} a_{2}}$ at $\boldsymbol{z}_{1}=\boldsymbol{z}_{2}=\mathbf{0}$ is diagonal in $R$ and $R^{\prime}$ and independent of the parton species. Following the construction in appendix A, one finds that the matrix $s$ in (3.25) is also diagonal in $R$ and $R^{\prime}$ and satisfies the relation

$$
R R_{s}(Y)=\sqrt{R R S(2 Y)}
$$

which is the analogue of (3.47) for SPS. The definition of DPDFs from unsubtracted collinear matrix elements in (3.29) thus turns into

$$
{ }^{R} F_{a_{1} a_{2}}\left(Y_{C}\right)=\lim _{Y_{L} \rightarrow-\infty} R R_{s}^{-1}\left(Y_{C}-Y_{L}\right)^{R} F_{\mathrm{us}, a_{1} a_{2}}\left(Y_{L}\right)
$$

for a right moving proton, and correspondingly for a left moving one. The kernel $K=\widehat{K}$ controlling the $Y$ dependence of $S(Y)$ and $s(Y)$ is of course also diagonal in $R$ and $R^{\prime}$. We denote it by ${ }^{R} J$ in order to avoid confusion with the evolution kernel for TMDs. In terms of the variable $\zeta$, the rapidity evolution of DPDFs is then given by

$$
\frac{\partial}{\partial \log \zeta}{ }^{R} F\left(x_{i}, \boldsymbol{y} ; \mu_{i}, \zeta\right)=\frac{1}{2}^{R} J\left(\boldsymbol{y} ; \mu_{i}\right)^{R} F\left(x_{i}, \boldsymbol{y} ; \mu_{i}, \zeta\right),
$$

where we have explicitly given all arguments of the functions. Since in the colour singlet channel ${ }^{11} S=1$, one has ${ }^{1} J=0$ for the corresponding evolution kernel, i.e. the collinear colour singlet DPDFs are independent of the rapidity parameter $\zeta$.

Let us now discuss renormalisation. Setting $\boldsymbol{z}_{1}=\boldsymbol{z}_{2}=\mathbf{0}$ in the soft factor $S$ and in the hadronic matrix elements $F_{\text {us }}$ induces additional UV divergences compared to the case 
where these distances are finite. The graphs requiring renormalisation are now not only vertex corrections and self energies, but also involve the exchange of hard gluons between partons or Wilson lines associated with the scattering amplitude and its complex conjugate. This is of course well known from the renormalisation of the twist-two operators that define ordinary PDFs, which is not purely multiplicative but involves a convolution in the momentum fraction $x$ and mixing between quark and gluon operators. In the renormalisation group equations for PDFs, splitting kernels $P_{a b}$ take the role of the anomalous dimensions $\gamma_{F, a}$ in the TMD case.

In the case of DPDFs we must pay particular attention to colour. Taking the twist-two operators (3.10) at $\boldsymbol{z}=\mathbf{0}$ and projecting their colour indices on a definite representation $R$ as in (4.18), we obtain the colour projected matrix element ${ }^{R} F_{\text {us }}$ according to (4.19). Due to gauge invariance, operators ${ }^{R} O_{a}$ belonging to different colour representations do not mix under renormalisation. To obtain the soft factor ${ }^{R R} S$, we evaluate the Wilson line products (3.16) at $\boldsymbol{z}=\mathbf{0}$ and project them on definite colour channels $R$ as $P_{\bar{R}}^{\underline{r} \underline{s}} O_{\bar{S}, a}^{\underline{r}, \underline{s}}$. Again, operators with different $R$ do not mix under renormalisation. The renormalisation of the soft factor remains multiplicative, because it is identical for quarks and gluons and because it does not depend on $x$.

With this in mind, one can repeat the arguments we developed in section 3.4 for the renormalisation of DTMDs and of their Collins-Soper kernels. One then obtains

$$
\frac{\partial}{\partial \log \mu_{1}}{ }^{R} J\left(\boldsymbol{y} ; \mu_{i}\right)=-{ }^{R} \gamma_{J}\left(\mu_{1}\right)
$$

for the Collins-Soper kernel and

$$
\frac{\partial}{\partial \log \mu_{1}}{ }^{R} F_{a_{1} a_{2}}\left(x_{i}, \boldsymbol{y} ; \mu_{i}, \zeta\right)=2 \sum_{b_{1}}{ }^{R} P_{a_{1} b_{1}}\left(x_{1}^{\prime} ; \mu_{1}, x_{1} \zeta / x_{2}\right) \underset{x_{1}}{\otimes}{ }^{R} F_{b_{1} a_{2}}\left(x_{1}^{\prime}, x_{2}, \boldsymbol{y} ; \mu_{i}, \zeta\right)
$$

for DPDFs, as well as analogous equations for the $\mu_{2}$ dependence. ${ }^{7}$ Let us emphasise that the evolution equation (5.24) for DPDFs depending on $\boldsymbol{y}$ is homogeneous. As discussed in $[27,30]$, an additional term for the splitting of one parton into two arises on the r.h.s. if one integrates the distributions over $\boldsymbol{y}$ or Fourier transforms them w.r.t. that variable. The resulting inhomogeneous equation has been extensively studied in the literature [87-91].

By construction, the kernels ${ }^{1} P_{a_{1} b_{1}}$ for the colour singlet sector are the ordinary DGLAP kernels for the evolution of PDFs. They are hence independent of $\zeta$, which is not the case in the other colour channels. Indeed, combining (5.22), (5.23), (5.24) and requiring equality of the derivatives $\partial /\left(\partial \log \mu_{i}\right) \partial /(\partial \log \zeta)^{R} F$ and $\partial /(\partial \log \zeta) \partial /\left(\partial \log \mu_{i}\right)^{R} F$, we find

and thus

$$
\frac{\partial}{\partial \log \zeta}{ }^{R} P_{a b}(x ; \mu, \zeta)=-\frac{1}{4} \delta_{a b} \delta(1-x)^{R} \gamma_{J}(\mu)
$$

$$
{ }^{R} P_{a b}(x ; \mu, \zeta)=-\frac{1}{2} \delta_{a b} \delta(1-x){ }^{R} \gamma_{J}(\mu) \log \frac{\sqrt{\zeta}}{\mu}+{ }^{R} P_{a b}\left(x ; \mu, \mu^{2}\right) .
$$

\footnotetext{
${ }^{7}$ Regarding factors of 2 , our convention for splitting kernels follows [65], so that $2 P$ takes the place of the anomalous dimension $\gamma_{F, a}$ in TMD evolution. The kernel ${ }^{R} P$ in (5.24) should not be confused with the colour projector $P_{R}$.
} 
These are the analogues of the relations (2.4) and (2.5) for the anomalous dimension $\gamma_{F, a}(\mu, \zeta)$ in TMD evolution.

We can make the $\zeta$ dependence of the DPDFs fully explicit in the form

$$
\begin{aligned}
{ }^{R} F_{a_{1} a_{2}}\left(x_{i}, \boldsymbol{y} ; \mu_{i}, \zeta\right)=\exp [ & -\int_{\mu_{0}}^{\mu_{1}} \frac{d \mu}{\mu}{ } \gamma_{J}(\mu) \log \frac{\sqrt{x_{1} \zeta / x_{2}}}{\mu}-\int_{\mu_{0}}^{\mu_{2}} \frac{d \mu}{\mu} \gamma_{J}(\mu) \log \frac{\sqrt{x_{2} \zeta / x_{1}}}{\mu} \\
& \left.+{ }^{R} J\left(\boldsymbol{y} ; \mu_{0}, \mu_{0}\right) \log \frac{\sqrt{\zeta}}{\sqrt{\zeta_{0}}}\right]{ }^{R} \widehat{F}_{a_{1} a_{2}, \mu_{0}, \zeta_{0}}\left(x_{i}, \boldsymbol{y} ; \mu_{i}\right),
\end{aligned}
$$

where $\widehat{F}$ is defined by the differential equation

$$
\begin{aligned}
\frac{\partial}{\partial \log \mu_{1}} & R \widehat{F}_{a_{1} a_{2}, \mu_{0}, \zeta_{0}}\left(x_{i}, \boldsymbol{y} ; \mu_{1}, \mu_{2}\right) \\
& =2 \sum_{b_{1}}{ }^{R} P_{a_{1} b_{1}}\left(x_{1}^{\prime} ; \mu_{1}, \mu_{1}^{2}\right) \underset{x_{1}}{\otimes} \widehat{F}_{b_{1} a_{2}, \mu_{0}, \zeta_{0}}\left(x_{1}^{\prime}, x_{2}, \boldsymbol{y} ; \mu_{1}, \mu_{2}\right)
\end{aligned}
$$

and its analogue for $\mu_{2}$, with the initial condition

$$
{ }^{R} \widehat{F}_{a_{1} a_{2}, \mu_{0}, \zeta_{0}}\left(x_{i}, \boldsymbol{y} ; \mu_{0}, \mu_{0}\right)={ }^{R} F_{a_{1} a_{2}}\left(x_{i}, \boldsymbol{y} ; \mu_{0}, \mu_{0}, \zeta_{0}\right) .
$$

Unlike $F$, the distribution $\widehat{F}$ evolves with $\zeta$ independent kernels. The correct scale dependence of (5.27) can be verified by inserting (5.25) into (5.24), and its $\zeta$ dependence can be verified by using (5.22) and (5.23).

The integrals over $\mu$ in (5.27) give rise to double logarithms, which have been exponentiated to a Sudakov factor in the solution of the evolution equation. Since the anomalous dimensions ${ }^{R} \gamma_{J}$ are positive for all $R \neq 1$ (see section 7.2.1), the contribution of all colour non-singlet DPDs to the cross section is Sudakov suppressed for sufficiently large hard scales. This was recognised long ago in [92] and confirmed in [28], where a simple numerical estimate was also given. In [28], scale and rapidity evolution was discussed for collinear DPS factorisation, keeping unsubtracted DPDs and an explicit soft factor in the cross section. The individual evolution equations hence differ between their approach and ours, but must of course give the same effects at the cross section level.

\subsection{Combining DPS with SPS}

The DPS cross section given in (5.1) is not a physical observable, because the same final state can also be produced by SPS. Dimensional analysis readily shows that DPS and SPS contribute to the TMD cross section at the same power in $1 / Q$. The distinction between the two mechanisms is in fact nontrivial, since there are Feynman graphs that contribute to both of them. These are graphs in which one parton splits into two, as shown in figure 4a. The kinematic region where the $q \bar{q}$ pairs are nearly collinear with their parent gluon is naturally described as DPS, with the splitting being included in the DPDs. By contrast, the region where the quarks and antiquarks have large transverse momenta (and hence large virtualities) is adequately described as a loop correction to SPS, with gluon TMDs describing the initial state of the hard scattering.

The interference between SPS and DPS has the same power behaviour in $1 / Q$ as SPS and DPS and hence should also be included in the cross section. Again, there is a double 


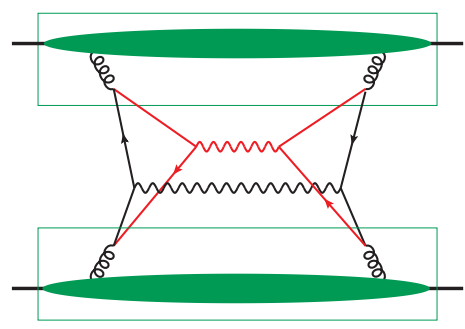

(a)

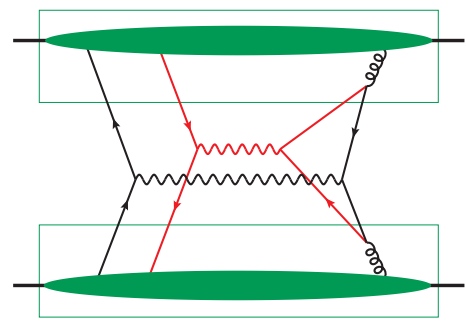

(b)

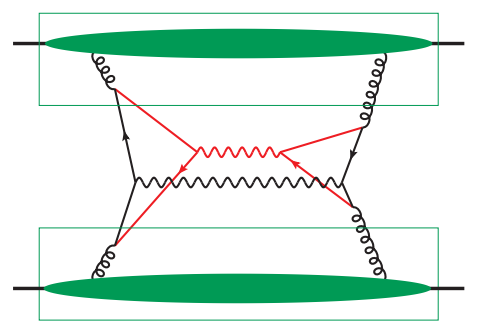

(c)

Figure 4. Graphs that contribute to several terms in the cross section (5.30). The rectangular boxes and oval blobs indicate the relevant hadronic matrix elements, depending on whether the $g \rightarrow q \bar{q}$ splittings are collinear or hard.

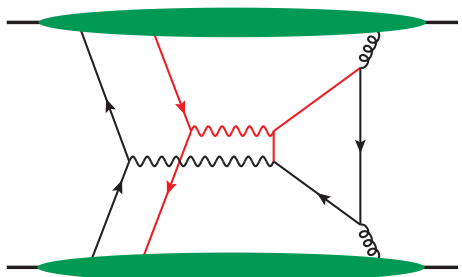

(a)

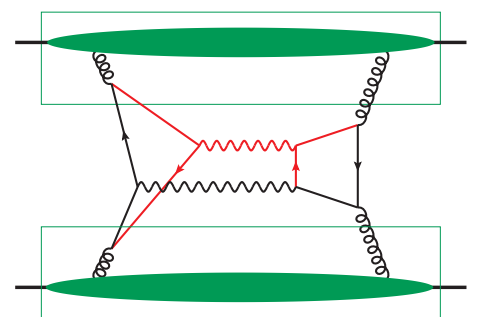

(b)

Figure 5. (a) A graph for the SPS/DPS interference that does not have overlap with DPS. (b) An SPS graph that has no overlap with DPS but with the SPS/DPS interference.

counting problem, since graphs like the one in figure $4 \mathrm{~b}$ contribute to either the interference term or to DPS, depending on whether the splitting takes place in the collinear or in the hard momentum region. Note that not all graphs are affected by double counting. The graphs in figure 5 for instance have no overlap with DPS, whereas there is overlap between SPS and the SPS/DPS interference in figure 5b.

A systematic formalism for separating the different contributions and for adding them in the cross section without double counting has been presented in [30]. In the following, we briefly recapitulate the essentials of this scheme for TMD factorisation, referring to that work for details. DTMDs and other hadronic matrix elements are used in the transverse position (rather than transverse momentum) representation. This is very convenient for treating Collins-Soper evolution, which is then multiplicative (rather than involving convolution integrals). The distinction between collinear and hard parton splitting then corresponds to the transverse distance between the partons being large or small, respectively.

As can be seen in figure $6 \mathrm{a}$, the relative distance between the two partons with momentum fractions $x_{1}$ and $x_{2}$ in a DTMD is $\boldsymbol{y}_{+}$for the partons in the amplitude and $\boldsymbol{y}_{-}$ for those in the conjugate amplitude, with the distances defined in (5.3). The contribution from small distances to the DPS cross section $\sigma_{\text {DPS }}$ is removed by the factors $\Phi\left(\nu \boldsymbol{y}_{+}\right)$and $\Phi\left(\nu \boldsymbol{y}_{-}\right)$in (5.2), which we discuss further at the end of this section. Corresponding factors 


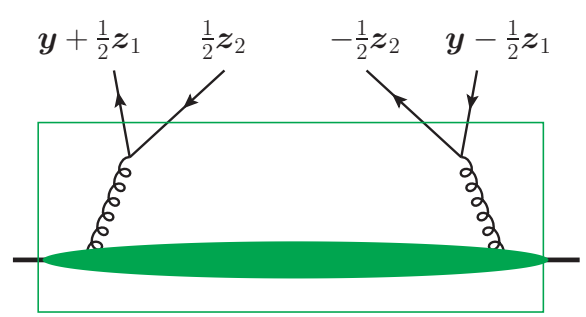

(a)

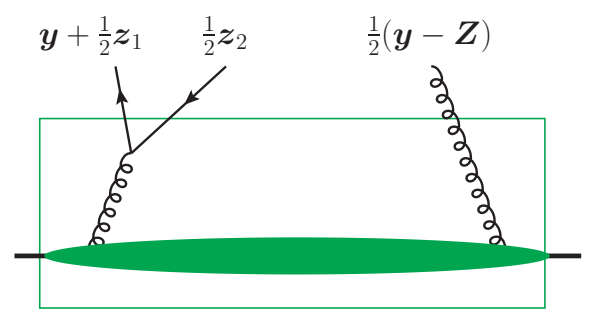

(b)

Figure 6. Transverse positions of the parton fields in parton splitting contributions to (a) the quark-antiquark DTMD and (b) the twist-three TMD $D_{q \bar{q} \mid g}$. Due to translation invariance, the latter depends only on $\boldsymbol{y}_{+}$and $\boldsymbol{Z}$. The positions in (b) correspond to equation (3.9) in [30].

are inserted in the interference $\sigma_{\mathrm{DPS} / \mathrm{SPS}}+\sigma_{\mathrm{SPS} / \mathrm{DPS}}$ between SPS and DPS. The SPS cross section $\sigma_{\text {SPS }}$ is not modified in this scheme and computed as usual from TMDs and hard scattering cross sections, as described for instance in [65]. The master formula for the overall cross section then reads

$$
\begin{aligned}
\sigma= & \sigma_{\mathrm{DPS}}+\left[\sigma_{\mathrm{DPS} / \mathrm{SPS}}-\sigma_{\mathrm{DPS}, y_{-} \rightarrow 0}+\sigma_{\mathrm{SPS} / \mathrm{DPS}}-\sigma_{\mathrm{DPS}, y_{+} \rightarrow 0}\right] \\
& +\left[\sigma_{\mathrm{SPS}}-\sigma_{\mathrm{DPS} / \mathrm{SPS}, y_{+} \rightarrow 0}-\sigma_{\mathrm{SPS} / \mathrm{DPS}, y_{-} \rightarrow 0}+\sigma_{\mathrm{DPS}, y_{ \pm} \rightarrow 0}\right],
\end{aligned}
$$

where all terms should be taken differential in $d x_{1} d x_{2} d \bar{x}_{1} d \bar{x}_{2} d^{2} \boldsymbol{q}_{1} d^{2} \boldsymbol{q}_{2}$. In this formula, the double counting problem between different contributions is solved by subtraction terms, which we will discuss shortly.

Let us start with the interference between DPS and SPS. As the colour and polarisation of the partons are not the main focus of the present section, we will not display these quantum numbers explicitly. The following equations hold for specific colour and helicity of each parton on the l.h.s. and with appropriate sums on the r.h.s. The indices $\alpha_{i}, \beta_{i}$ will just denote the parton species. Up to kinematic and numeric factors, the interference cross section is given by

$$
\begin{gathered}
\frac{d \sigma_{\mathrm{DPS} / \mathrm{SPS}}}{d x_{1} d x_{2} d \bar{x}_{1} d \bar{x}_{2} d^{2} \boldsymbol{q}_{1} d^{2} \boldsymbol{q}_{2}} \propto \sum_{\substack{\alpha_{1}, \alpha_{2}, \alpha_{0} \\
\beta_{1}, \beta_{2}, \beta_{0}}} H_{\alpha_{1} \beta_{1}} H_{\alpha_{2} \beta_{2}} H_{\alpha_{0} \beta_{0}}^{*} \int d^{2} \boldsymbol{Z} d^{2} \boldsymbol{y}_{+} e^{-i\left(\boldsymbol{q}_{1}+\boldsymbol{q}_{2}\right) \boldsymbol{Z}} \\
\times e^{-i\left(\boldsymbol{q}_{1}-\boldsymbol{q}_{2}\right) \boldsymbol{y}_{+} / 2} \Phi\left(\nu \boldsymbol{y}_{+}\right) D_{\beta_{1} \beta_{2} \mid \beta_{0}}\left(\bar{x}_{i}, \boldsymbol{y}_{+}, \boldsymbol{Z}\right) D_{\alpha_{1} \alpha_{2} \mid \alpha_{0}}\left(x_{i}, \boldsymbol{y}_{+}, \boldsymbol{Z}\right)
\end{gathered}
$$

for DPS in the amplitude, and by

$$
\begin{gathered}
\frac{d \sigma_{\mathrm{SPS} / \mathrm{DPS}}}{d x_{1} d x_{2} d \bar{x}_{1} d \bar{x}_{2} d^{2} \boldsymbol{q}_{1} d^{2} \boldsymbol{q}_{2}} \propto \sum_{\substack{\alpha_{0}, \alpha_{1}, \alpha_{2} \\
\beta_{0}, \beta_{1}, \beta_{2}}} H_{\alpha_{0} \beta_{0}} H_{\alpha_{1} \beta_{1}}^{*} H_{\alpha_{2} \beta_{2}}^{*} \int d^{2} \boldsymbol{Z} d^{2} \boldsymbol{y}_{-} e^{-i\left(\boldsymbol{q}_{1}+\boldsymbol{q}_{2}\right) \boldsymbol{Z}} \\
\times e^{i\left(\boldsymbol{q}_{1}-\boldsymbol{q}_{2}\right) \boldsymbol{y}_{-} / 2} \Phi\left(\nu \boldsymbol{y}_{-}\right) D_{\beta_{0} \mid \beta_{1} \beta_{2}}\left(\bar{x}_{i}, \boldsymbol{y}_{-}, \boldsymbol{Z}\right) D_{\alpha_{0} \mid \alpha_{1} \alpha_{2}}\left(x_{i}, \boldsymbol{y}_{-}, \boldsymbol{Z}\right)
\end{gathered}
$$

for DPS in the complex conjugate amplitude. Here $D_{\alpha_{1} \alpha_{2} \mid \alpha_{0}}$ is a twist-three TMD with two partons $\alpha_{1} \alpha_{2}$ in the amplitude and one parton $\alpha_{0}$ in the conjugate amplitude. Its definition is similar to the one of DTMDs, with three instead of four parton operators in 
the hadronic matrix element. The transverse positions of the partons are as indicated in figure $6 \mathrm{~b}$. In $D_{\alpha_{0} \mid \alpha_{1} \alpha_{2}}$, the roles of amplitude and conjugate amplitude are interchanged. $H_{\alpha_{1} \beta_{1}}$ and $H_{\alpha_{2} \beta_{2}}$ are the hard scattering amplitudes for the DPS processes, whilst $H_{\alpha_{0} \alpha_{0}}$ is the amplitude for the hard SPS interaction.

$\sigma_{\text {DPS/SPS }}$ receives a contribution from the graph in figure $4 \mathrm{~b}$ without the rectangular boxes. The quark loop in the conjugate amplitude includes an integration over the full phase space, including the region where the $g \rightarrow q \bar{q}$ splittings become collinear. By a Fourier transform, one can rewrite the transverse part of the loop integration as an integral over $\boldsymbol{y}_{-}$, after which the region of collinear splitting corresponds to large $\boldsymbol{y}_{-}$. This contribution is already included in $\sigma_{\text {DPS }}$, so that a subtraction term is necessary to prevent double counting. It reads

$$
\begin{aligned}
& \frac{d \sigma_{\mathrm{DPS}, y_{-} \rightarrow 0}}{d x_{1} d x_{2} d \bar{x}_{1} d \bar{x}_{2} d^{2} \boldsymbol{q}_{1} d^{2} \boldsymbol{q}_{2}} \propto \sum_{\substack{\alpha_{1}, \alpha_{2} \\
\beta_{1}, \beta_{2}}} H_{\alpha_{1} \beta_{1}} H_{\alpha_{2} \beta_{2}} H_{\alpha_{1} \beta_{1}}^{*} H_{\alpha_{2} \beta_{2}}^{*} \\
& \quad \times \int d^{2} \boldsymbol{Z} d^{2} \boldsymbol{y}_{+} d^{2} \boldsymbol{y}_{-} e^{-i\left(\boldsymbol{q}_{1}+\boldsymbol{q}_{2}\right) \boldsymbol{Z}-i\left(\boldsymbol{q}_{1}-\boldsymbol{q}_{2}\right)\left(\boldsymbol{y}_{+}-\boldsymbol{y}_{-}\right) / 2} \Phi\left(\nu \boldsymbol{y}_{+}\right) \Phi\left(\nu \boldsymbol{y}_{-}\right) \\
& \quad \times F_{\beta_{1} \beta_{2}, y_{-} \rightarrow 0}\left(\bar{x}_{i}, \boldsymbol{y}_{ \pm}, \boldsymbol{Z}\right) F_{\alpha_{1} \alpha_{2}, y_{-} \rightarrow 0}\left(x_{i}, \boldsymbol{y}_{ \pm}, \boldsymbol{Z}\right)
\end{aligned}
$$

Compared with the DPS cross section given by (5.1) and (5.2), we have traded the arguments $\boldsymbol{z}_{i}, \boldsymbol{y}$ of the DTMDs for $\boldsymbol{y}_{ \pm}, \boldsymbol{Z}$ and correspondingly changed the integration according to (5.5). For simplicity we write $y_{-} \rightarrow 0$ instead of $\boldsymbol{y}_{-} \rightarrow \mathbf{0}$ in subscripts. The DTMDs in (5.33) correspond to the rectangular boxes in figure $4 \mathrm{~b}$ and are given by

$$
F_{\alpha_{1} \alpha_{2}, y_{-} \rightarrow 0}\left(x_{i}, \boldsymbol{y}_{ \pm}, \boldsymbol{Z}\right)=\frac{\boldsymbol{y}_{-}^{l^{\prime}}}{\boldsymbol{y}_{-}^{2}}\left[U_{\alpha_{0} \rightarrow \alpha_{1} \alpha_{2}}^{l^{\prime}}\left(x_{i}\right)\right]^{*} D_{\alpha_{1} \alpha_{2} \mid \alpha_{0}}\left(x_{i}, \boldsymbol{y}_{+}, \boldsymbol{Z}\right)+\mathcal{O}\left(\alpha_{s}^{3 / 2}\right) .
$$

This notation reflects that in the limit $\boldsymbol{y}_{-} \rightarrow \mathbf{0}$ the DTMD is dominated by the contribution where the two partons $\alpha_{1}$ and $\alpha_{2}$ in the conjugate amplitude originate from the splitting of a single parton $\alpha_{0}$. The lowest order perturbative splitting kernel $U$ includes a factor of $\sqrt{\alpha_{s}}$. For given $\alpha_{1}$ and $\alpha_{2}$, the parton species $\alpha_{0}$ is fixed at this order, but its helicity must be summed over. As shown in [30], the combination $\sigma_{\mathrm{DPS}}+\sigma_{\mathrm{DPS} / \mathrm{SPS}}-\sigma_{\mathrm{DPS}, y_{-} \rightarrow 0}$ correctly treats the contribution of graph $4 \mathrm{~b}$ to the cross section, for small and for large $\boldsymbol{y}_{-}$, and without double counting. We will see in more detail how this happens in section 6.5. The other subtraction term in the first line of (5.30) is given by expressions analogous to (5.33) and (5.34), with $\boldsymbol{y}_{-}$replaced by $\boldsymbol{y}_{+}$and the roles of amplitude and conjugate amplitude being interchanged.

The subtraction terms $\sigma_{\mathrm{DPS} / \mathrm{SPS}, y_{+} \rightarrow 0}$ and $\sigma_{\mathrm{SPS} / \mathrm{DPS}, y_{-} \rightarrow 0}$ in the second line of (5.30) are obtained from (5.31) and (5.32) by replacing all distributions $D$ with their perturbative splitting approximation. For the case shown in figure $6 \mathrm{~b}$, this is given by

$$
D_{\alpha_{1} \alpha_{2} \mid \alpha_{0}, y_{+} \rightarrow 0}\left(x_{i}, \boldsymbol{y}_{+}, \boldsymbol{Z}\right)=\frac{\boldsymbol{y}_{+}^{l}}{\boldsymbol{y}_{+}^{2}} U_{\alpha_{0} \rightarrow \alpha_{1} \alpha_{2}}^{l}\left(x_{i}\right) f_{\alpha_{0}}\left(x_{1}+x_{2}, \boldsymbol{Z}\right)+\mathcal{O}\left(\alpha_{s}^{3 / 2}\right)
$$

where on the r.h.s. we have a single parton TMD and the same splitting kernel $U$ as in (5.34). An analogous expression holds for the limit $\boldsymbol{y}_{-} \rightarrow \mathbf{0}$ of $D_{\alpha_{0} \mid \alpha_{1} \alpha_{2}}$. The combination 
$\sigma_{\mathrm{DPS} / \mathrm{SPS}}+\sigma_{\mathrm{SPS}}-\sigma_{\mathrm{DPS} / \mathrm{SPS}, y_{+} \rightarrow 0}$ correctly represents graph $4 \mathrm{c}$ in the region where the quark loop in the conjugate amplitude is hard.

Finally, the term $\sigma_{\mathrm{DPS}, y_{ \pm} \rightarrow 0}$ in (5.30) ensures the correct treatment of the case when both quark loops in graph $4 \mathrm{a}$ are in the collinear region. This term is given by the DPS cross section with each DTMD approximated for the regime where both parton pairs are produced by perturbative splitting:

$$
F_{\alpha_{1} \alpha_{2}, y_{ \pm} \rightarrow 0}\left(x_{i}, \boldsymbol{y}_{ \pm}, \boldsymbol{Z}\right)=\frac{\boldsymbol{y}_{+}^{l}}{\boldsymbol{y}_{+}^{2}} \frac{\boldsymbol{y}_{-}^{l^{\prime}}}{\boldsymbol{y}_{-}^{2}} U_{\alpha_{0} \rightarrow \alpha_{1} \alpha_{2}}^{l}\left(x_{i}\right)\left[U_{\alpha_{0} \rightarrow \alpha_{1} \alpha_{2}}^{l^{\prime}}\left(x_{i}\right)\right]^{*} f_{\alpha_{0}}\left(x_{1}+x_{2}, \boldsymbol{Z}\right)+\mathcal{O}\left(\alpha_{s}^{2}\right) .
$$

This is illustrated in figure 6a and can be obtained by inserting the expression (5.35) into (5.34). Notice that $\sigma_{\mathrm{DPS}, y_{ \pm} \rightarrow 0}$ appears with a plus rather than a minus sign in (5.30). This is a consequence of the recursive nature of double counting subtractions, as explained in section 4.2 of [30].

To make contact with our notation in section 6.3.1, we rewrite (5.36) in terms of a single splitting kernel $\mathrm{as}^{8}$

$$
{ }^{R} F_{a_{1} a_{2}, y_{ \pm} \rightarrow 0}\left(x_{i}, \boldsymbol{z}_{i}, \boldsymbol{y}\right)=\frac{\boldsymbol{y}_{+}^{l} \boldsymbol{y}_{-}^{l^{\prime}}}{\boldsymbol{y}_{+}^{2} \boldsymbol{y}_{-}^{2}} \sum_{a_{0}} \frac{\alpha_{s}}{2 \pi^{2}}{ }^{R} T_{a_{0} \rightarrow a_{1} a_{2}}^{l l^{\prime}}\left(\frac{x_{1}}{x_{1}+x_{2}}\right) \frac{f_{a_{0}}\left(x_{1}+x_{2}, \boldsymbol{Z}\right)}{x_{1}+x_{2}}+\mathcal{O}\left(\alpha_{s}^{2}\right),
$$

where we have restored the dependence on the colour representation and the notation with labels $a_{i}$ that specify parton species and polarisation. For transverse quark or linear gluon polarisation, the parton distributions and the kernel $T_{a_{0} \rightarrow a_{1} a_{2}}$ carry additional transverse indices, which are not displayed here (see section 7.4). The sum over $a_{0}$ reflects the fact that there are TMDs for these polarisation states, even if the proton is unpolarised.

We can now discuss the role of the function $\Phi$ introduced earlier. The short-distance behaviour of the DTMDs and twist-three distributions, given in (5.34) and (5.35), results in an integral

$$
\int_{\left|\boldsymbol{y}_{+}\right| \ll 1 / \Lambda} \frac{d^{2} \boldsymbol{y}_{+}}{\boldsymbol{y}_{+}^{2}} e^{-i\left(\boldsymbol{q}_{1}-\boldsymbol{q}_{2}\right) \boldsymbol{y}_{+} / 2} \Phi\left(\nu \boldsymbol{y}_{+}\right)
$$

and its analogue for $\boldsymbol{y}_{-}$in the cross section formulae for DPS and for the SPS/DPS interference. The function $\Phi$ must satisfy $\Phi(\boldsymbol{u}) \rightarrow 0$ for $\boldsymbol{u} \rightarrow \mathbf{0}$ to ensure that these integrals converge at small distances (rather than having a logarithmic divergence). In order to keep the regions of large $\boldsymbol{y}_{+}$and $\boldsymbol{y}_{-}$unaffected, one should furthermore have $\Phi(\boldsymbol{u}) \rightarrow 1$ for $|\boldsymbol{u}| \gg 1$. A simple choice for $\Phi(\boldsymbol{u})$ is a step function in $|\boldsymbol{u}|$, which corresponds to a hard cutoff on the $\boldsymbol{y}_{ \pm}$integrals.

The integral (5.38) goes like $\log \left(\nu / q_{T}\right)$ and thus depends on the artificial parameter $\nu$ that controls which distances $\boldsymbol{y}_{ \pm}$are included in what we define to be DPS rather than SPS. The $\nu$ dependence of the different terms in the cross section (5.30) cancels to the perturbative order of the calculation, in close analogy with the familiar case of renormalisation and factorisation scale dependence. As discussed in [30], a suitable choice for $\nu$ is the lowest hard

\footnotetext{
${ }^{8}$ The convention for $T^{j j^{\prime}}$ here is the same as in [30] (arXiv version 2) and differs from the one in [27] as specified in section 7.4 .
} 
scale, $\min \left(Q_{1}, Q_{2}\right)$. With this choice, $\sigma_{\text {DPS }}$ contains a squared logarithm $\log ^{2}\left(Q / q_{T}\right)$, where we recall that $Q$ denotes the generic size of $Q_{1}$ and $Q_{2}$. After the subtractions in the first line of (5.30), the SPS/DPS interference contains only a single $\log \left(Q / q_{T}\right)$ and the subtracted SPS term in the second line has no such logarithm at the corresponding order in $\alpha_{s}$. This has an important consequence. If one is satisfied with leading logarithmic accuracy, then the terms in square brackets in (5.30) can be neglected at the order in $\alpha_{s}$ where they have overlap with DPS, because at that order it is $\sigma_{\text {DPS }}$ that has the highest power of $\log \left(Q / q_{T}\right)$.

We should note that the structure of the higher-order terms in (5.34), (5.35) and (5.36) is currently unknown. In particular, it cannot be excluded that singularities will appear at points other than $\boldsymbol{y}_{+}=\mathbf{0}$ and $\boldsymbol{y}_{-}=\mathbf{0}$. The treatment of ultraviolet divergences and of the associated double counting issues may thus be more involved beyond the leading order discussed here.

To conclude this section, we briefly discuss the scale evolution of the twist-three TMDs $D$. As already noted, they are defined in terms of hadronic matrix elements, with three parton fields and the corresponding Wilson lines instead of the four fields we have in the unsubtracted DTMDs in (3.2). The three parton fields are at different transverse positions (if all three positions are equal, one has collinear twist-three distributions). It is easy to see that the analysis of factorisation for the SPS/DPS interference will yield a soft factor, with three products of Wilson lines associated with the three parton fields in the hadronic matrix elements (rather than four products of Wilson lines as in figure 1). One can readily adapt the discussion in sections 3.2 to 3.4 to this case and absorb the soft factor into the twist-three distributions. The colour algebra is much simpler than in the DPS case, with a single colour representation in the $q \bar{q} g$ channel, and two for the channel with three gluons (one constructed with $f^{a b c}$ and the other with $d^{a b c}$ ). The rapidity dependence of $D_{\alpha_{1} \alpha_{2} \mid \alpha_{0}}$ is finally given by a Collins-Soper equation as in (5.11) with a kernel $K_{\alpha_{1} \alpha_{2} \mid \alpha_{0}}$. If one takes different renormalisation scales $\mu_{1}, \mu_{2}$ and $\mu_{0}$ for the three parton legs, then the dependence of $D$ and $K$ on these scales is given by the analogues of (5.7) and (5.12), with $\gamma_{K, a}$ replaced by $\gamma_{K, a} / 2$ and $\gamma_{F, a}$ by $\gamma_{E, a}$ or $\left(\gamma_{E, a}\right)^{*}$ as discussed at the end of section 3.4. The rapidity parameter $\zeta$ is defined by (5.8) and must be properly rescaled in the argument of $\gamma_{F, a}$, namely by $x_{1} \zeta / x_{2}, x_{2} \zeta / x_{1}$ and $\left(x_{1}+x_{2}\right)^{2} \zeta /\left(x_{1} x_{2}\right)$ for the partons with momentum fractions $x_{1}, x_{2}$ and $x_{1}+x_{2}$, respectively.

\section{Matching for small but perturbative transverse momenta}

In the multi-scale regime $\Lambda \ll q_{T} \ll Q$, where the transverse momenta $\left|\boldsymbol{q}_{i}\right|$ are small compared with $Q_{i}$ but large compared with the scale $\Lambda$ of nonperturbative physics, the DPS cross section, as well as its combination with SPS can be considerably simplified. The reason is that the additional scale $q_{T}$ allows for more perturbative calculations, which greatly enhances the predictive power of the theory.

We start our analysis by discussing the different transverse distance regions in the DPS factorisation formula and find that two distinct regions of $\boldsymbol{y}$ are relevant. The corresponding short-distance expansions of DTMDs are derived in sections 6.2 and 6.3, respectively. In section 6.4 we discuss the master formula (6.51) for combining the two types of expansion in 
the cross section, where care must be taken to avoid double counting. After this, we revisit the combination between DPS and SPS just discussed in section 5.3. In section 6.6 we go through the perturbative ingredients that are necessary for a given resummation order as specified in table 3; their availability determines the perturbative accuracy of cross section computations.

\subsection{Regions of transverse momenta and distances}

In the DPS cross section (5.1), contributions from distances $\left|\boldsymbol{z}_{i}\right| \gg 1 /\left|\boldsymbol{q}_{i}\right|$ are suppressed by oscillations of the Fourier exponent $e^{-i \boldsymbol{q}_{1} \boldsymbol{z}_{1}-i \boldsymbol{q}_{2} \boldsymbol{z}_{2}}$. If $\boldsymbol{q}_{1}$ and $\boldsymbol{q}_{2}$ are sufficiently large, this keeps $\boldsymbol{z}_{1}$ and $\boldsymbol{z}_{2}$ in the region where perturbation theory has predictive power for the dependence on these variables.

Care is required if $\boldsymbol{q}_{1}$ and $\boldsymbol{q}_{2}$ are perturbatively large but $\left|\boldsymbol{q}_{1}+\boldsymbol{q}_{2}\right|$ is of order $\Lambda$. According to (5.5) the sum $\left|\boldsymbol{z}_{1}+\boldsymbol{z}_{2}\right|$ can then reach large values of order $1 / \Lambda$, as long as $\left|\boldsymbol{z}_{1}-\boldsymbol{z}_{2}\right|$ remains small. The individual oscillations of $e^{-i \boldsymbol{q}_{1} \boldsymbol{z}_{1}}$ and $e^{-i \boldsymbol{q}_{2} \boldsymbol{z}_{2}}$ cancel in that case. Likewise, $\left|\boldsymbol{z}_{1}-\boldsymbol{z}_{2}\right|$ can become as large as $1 / \Lambda$ if $\boldsymbol{q}_{1}$ and $\boldsymbol{q}_{2}$ are perturbatively large but $\left|\boldsymbol{q}_{1}-\boldsymbol{q}_{2}\right|$ is of order $\Lambda$. The perturbative splitting form (5.37) of DTMDs has a factorised dependence on the variables $\boldsymbol{z}_{1}+\boldsymbol{z}_{2}$ and $\boldsymbol{z}_{1}-\boldsymbol{z}_{2}$, and the short-distance expansion (6.38) we will derive from it requires both distances to be small. We therefore do not consider the particular phase space regions just mentioned and require $\left|\boldsymbol{q}_{1}\right|$ and $\left|\boldsymbol{q}_{2}\right|$ as well as $\left|\boldsymbol{q}_{1}+\boldsymbol{q}_{2}\right|$ and $\left|\boldsymbol{q}_{1}-\boldsymbol{q}_{2}\right|$ to be much larger than $\Lambda$. The dominant contribution to the $\boldsymbol{z}_{1}$ and $\boldsymbol{z}_{2}$ integrals in the cross section formula (5.1) is then in the perturbative region. Even with this requirement, one could still consider a region where $\left|\boldsymbol{q}_{1}+\boldsymbol{q}_{2}\right|$ is much smaller than $\left|\boldsymbol{q}_{1}\right|$ and $\left|\boldsymbol{q}_{2}\right|$. This multi-scale regime has been discussed in section 5.2.1 of [27] and in [47, 48]. We shall not investigate it in the present work.

Other observables in which sensitivity to large $\boldsymbol{z}_{1}$ and $\boldsymbol{z}_{2}$ can be avoided are obtained by integrating the cross section over one or both of $\boldsymbol{q}_{1}$ and $\boldsymbol{q}_{2}$ up to $q_{\max , 1}$ and $q_{\max , 2}$ respectively. In

$$
\int d^{2} \boldsymbol{q} \theta\left(q_{\max }^{2}-\boldsymbol{q}^{2}\right) \int \frac{d^{2} \boldsymbol{z}}{(2 \pi)^{2}} e^{-i \boldsymbol{q} \boldsymbol{z}} W(\boldsymbol{z}, \ldots)=q_{\max } \int \frac{d^{2} \boldsymbol{z}}{2 \pi|\boldsymbol{z}|} J_{1}\left(q_{\max }|\boldsymbol{z}|\right) W(\boldsymbol{z}, \ldots)
$$

the region of $|\boldsymbol{z}| \gg 1 / q_{\max }$ is damped by oscillations, as it is by the exponential factors in the differential cross section. To avoid that the product of the two Bessel functions has a non-oscillating component for $\left|\boldsymbol{z}_{1}\right| \approx\left|\boldsymbol{z}_{2}\right|$, one must take $q_{\max , 1} \neq q_{\max , 2}$.

We henceforth assume that both $\left|\boldsymbol{z}_{1}\right|$ and $\left|\boldsymbol{z}_{2}\right|$ are small, namely of order $1 / q_{T}$. Since $\boldsymbol{y}$ is integrated over without any Fourier exponent in (5.1) we must still consider the two cases $|\boldsymbol{y}| \sim 1 / \Lambda$ and $|\boldsymbol{y}| \sim 1 / q_{T}$, which are referred to as large $\boldsymbol{y}$ and small $\boldsymbol{y}$ in the following. For even smaller $|\boldsymbol{y}| \ll 1 / q_{T}$, the approximations for DPS are not valid and the subtraction formalism sketched in section 5.3 comes into play. The short-distance expansion of DPDs in the two DPS regions just mentioned is quite different and will now be discussed in turn.

\subsection{The large- $y$ region}

For small $\boldsymbol{z}_{1}, \boldsymbol{z}_{2}$ and large $\boldsymbol{y}$, the DTMD $F\left(x_{i}, \boldsymbol{z}_{i}, \boldsymbol{y}\right)$ can be computed in terms of DPDFs $F\left(x_{i}, \boldsymbol{y}\right)$, in close analogy to the case of single parton distributions. This is commonly 
referred to as "matching". For DTMDs there are complications from colour, but our results from section 4.4 lead to considerable simplifications. Generalising the relations (2.9) and (2.11) between TMDs and PDFs, we can derive the general matching equation (6.11) and the explicit solution (6.22) of the evolution equations for DTMDs. This solution is promoted to the cross section level in (6.24). We emphasise that the simple structure of (6.22) and (6.24) is due to the additive form (6.16) of the Collins-Soper matrix kernel in the short-distance limit considered here.

\subsubsection{Short-distance expansion}

We now derive the approximation of DTMDs in the region $\left|\boldsymbol{z}_{1}\right|,\left|\boldsymbol{z}_{2}\right| \ll 1 / \Lambda$, with $\boldsymbol{y}$ kept fixed at a value such that $\left|\boldsymbol{z}_{1}\right|,\left|\boldsymbol{z}_{2}\right| \ll|\boldsymbol{y}|$. Corrections to this approximation are suppressed by powers of $\left|\boldsymbol{z}_{i}\right| \Lambda$ and of $\left|\boldsymbol{z}_{i}\right| /|\boldsymbol{y}|$. To make the analogy between DTMDs and single parton TMDs transparent, we formulate the matching in terms of operator product expansions around $\boldsymbol{z}_{i}=\mathbf{0}$.

Let us start with the expansion of the soft factor. We need the small- $\boldsymbol{z}$ limit of the operators $O_{S, a}(\boldsymbol{y}, \boldsymbol{z})$ defined in (3.16), which contain two Wilson line pairs separated by a transverse distance $\boldsymbol{z}$. The colour structure of the short-distance expansion can be deduced from the corresponding Feynman graphs. To minimise the number of eikonal propagators that carry large momentum, interactions at the hard scale $1 /|\boldsymbol{z}|$ must take place closest to the points where the left- and right-moving Wilson lines meet, as shown in figure 7 . In terms of the operators in (3.13) and (3.16), we thus find the structure

$$
\left[O_{S, a}(\boldsymbol{y}, \boldsymbol{z})\right]^{\underline{r}, \underline{u}}=C_{S, a}^{\underline{s} \underline{t}}(\boldsymbol{z})\left[O_{S, a}(\boldsymbol{y})\right]_{\underline{\underline{s}}, \underline{t}}^{\underline{r}, \underline{u}}
$$

with a short-distance coefficient $C_{S, a}$ that can be computed in perturbation theory. Here and in the following, the absence of the argument $\boldsymbol{z}$ in operators or functions means that one has set $\boldsymbol{z}=\mathbf{0}$. The analogue of this convention will be used for arguments $\boldsymbol{z}_{i}$. Introducing colour projected coefficients ${ }^{R} C_{S, a}=P_{R}^{\underline{s} \underline{t}} C_{S, a}^{\underline{s} \underline{t}} / m(R)$ and using (4.11), we obtain

$$
\begin{aligned}
{\left[O_{S, a}(\boldsymbol{y}, \boldsymbol{z})\right]^{\underline{r}, \underline{u}} } & =\sum_{R}{ }^{R} C_{S, a}(\boldsymbol{z}) P_{\bar{R}}^{\underline{s} \underline{t}}\left[O_{S, a}(\boldsymbol{y})\right]_{\underline{\underline{s}}, \underline{\underline{t}}}^{\underline{r}, \underline{u}} \\
& =\sum_{R}{ }^{R} C_{S, a}(\boldsymbol{z}) P_{\bar{R}}^{\underline{r} \underline{s}} P_{\bar{R}}^{\underline{u} \underline{t}}\left[O_{S, a}(\boldsymbol{y})\right]_{\underline{\underline{s}}, \underline{\underline{v}} \underline{\underline{v}}},
\end{aligned}
$$

where the second step is completely analogous to the derivation of the relation (4.42) between the extended soft factor and the usual one. Taking the vacuum expectation value of $O_{S, a_{1}}\left(\boldsymbol{y}, \boldsymbol{z}_{1}\right) O_{S, a_{2}}\left(\mathbf{0}, \boldsymbol{z}_{2}\right)$ with appropriate colour projections, we obtain

$$
{ }^{R R^{\prime}} S_{a_{1} a_{2}}\left(\boldsymbol{z}_{i}, \boldsymbol{y}\right)={ }^{R} C_{S, a_{1}}\left(\boldsymbol{z}_{1}\right)^{R} C_{S, a_{2}}\left(\boldsymbol{z}_{2}\right){ }^{R R} S(\boldsymbol{y}) \delta_{R R^{\prime}}
$$

For computing the short-distance coefficients, it will be convenient to contract (6.3) with $P_{R^{\prime}}^{\underline{r}}$, which gives

$$
P_{R}^{\underline{r} \underline{u}}\left[O_{S, a}(\boldsymbol{y}, \boldsymbol{z})\right]^{\underline{r}, \underline{u}}={ }^{R} C_{S, a}(\boldsymbol{z}) P_{R}^{\underline{r}} \underline{u}\left[O_{S, a}(\boldsymbol{y})\right]^{\underline{r}, \underline{u}} .
$$




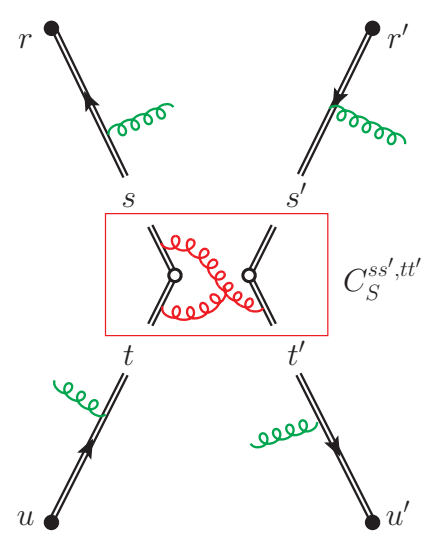

Figure 7. Interactions of Wilson lines in the operator $O_{S, a}(\boldsymbol{y}, \boldsymbol{z})$ in the limit of small $\boldsymbol{z}$. Shortdistance interactions are located in the box at the centre, whereas gluons outside the box indicate possible interactions with other Wilson lines at distances much larger than $\boldsymbol{z}$. In the soft factor $S_{a_{1} a_{2}}\left(\boldsymbol{z}_{i}, \boldsymbol{y}\right)$ these distances are of order $\boldsymbol{y}$ for small $\boldsymbol{z}_{1}$ and $\boldsymbol{z}_{2}$.

For approximating the unsubtracted collinear matrix element $F_{\text {us }}$, we need the small- $\boldsymbol{z}$ limit of the parton operators in (3.10). We use their projections (4.18) on definite colour representations, which cannot mix with each other due to gauge invariance. The shortdistance expansion involves a convolution integral over momentum fractions and reads ${ }^{9}$

$$
{ }^{R} O \frac{r}{a}(x, \boldsymbol{y}, \boldsymbol{z})=\sum_{b}{ }^{R} C_{\mathrm{us}, a b}\left(x^{\prime}, \boldsymbol{z}\right) \underset{x}{\otimes}{ }^{R} O_{b}^{\underline{r}}\left(x^{\prime}, \boldsymbol{y}\right) .
$$

If $a$ or $b$ refer to transverse quark or linear gluon polarisation, then the associated operators carry additional transverse indices, and the same holds of course for $C_{\mathrm{us}, a b}$. Taking matrix elements as in (4.19), we obtain an approximation

$$
{ }^{R} F_{\mathrm{us}, a_{1} a_{2}}\left(x_{i}, \boldsymbol{z}_{i}, \boldsymbol{y}\right)=\sum_{b_{1}, b_{2}}{ }^{R} C_{\mathrm{us}, a_{1} b_{1}}\left(x_{1}^{\prime}, \boldsymbol{z}_{1}\right) \underset{x_{1}}{\otimes} C_{\mathrm{us}, a_{2} b_{2}}\left(x_{2}^{\prime}, \boldsymbol{z}_{2}\right) \underset{x_{2}}{\otimes}{ }^{R} F_{\mathrm{us}, b_{1} b_{2}}\left(x_{i}^{\prime}, \boldsymbol{y}\right)
$$

of unsubtracted DTMDs in terms of unsubtracted DPDFs and perturbative coefficients $C_{\mathrm{us}, a b}(x, \boldsymbol{z})$.

We now combine the soft factor with the unsubtracted DTMD. Using the relation (5.20) between $s$ and $S$, we obtain

$$
{ }^{R R^{\prime}} S_{a_{1} a_{2}}\left(\boldsymbol{z}_{i}, \boldsymbol{y} ; \mu_{i}, Y\right)=\sqrt{{ }^{R} C_{S, a_{1}}\left(\boldsymbol{z}_{1} ; \mu_{1}, 2 Y\right)} \sqrt{{ }^{R} C_{S, a_{2}}\left(\boldsymbol{z}_{2} ; \mu_{2}, 2 Y\right)}{ }^{R} s\left(\boldsymbol{y} ; \mu_{i}, Y\right) \delta_{R R^{\prime}}
$$

from (6.4), where we have restored the dependence on renormalisation scales and rapidities. Combining this with (6.7) and using the definition (4.23) of DPDs, we get

$$
\begin{aligned}
{ }^{R} F_{a_{1} a_{2}}\left(x_{i}, \boldsymbol{z}_{i}, \boldsymbol{y} ; \mu_{i}, Y_{C}\right)= & \sum_{b_{1}, b_{2}}{ }^{R} C_{a_{1} b_{1}}\left(x_{1}^{\prime}, \boldsymbol{z}_{1} ; \mu_{1}, Y_{C}\right) \underset{x_{1}}{\otimes}{ }^{R} C_{a_{2} b_{2}}\left(x_{2}^{\prime}, \boldsymbol{z}_{2} ; \mu_{2}, Y_{C}\right) \\
& \underset{x_{2} Y_{L} \rightarrow-\infty}{\otimes} \lim ^{R} F_{\mathrm{us}, b_{1} b_{2}}\left(x_{i}^{\prime}, \boldsymbol{y} ; \mu_{i}, Y_{L}\right) /{ }^{R R} s\left(\boldsymbol{y} ; \mu_{i}, Y_{C}-Y_{L}\right)
\end{aligned}
$$

\footnotetext{
${ }^{9}$ The lower limit of the convolution in (6.6) is $x^{\prime}=0$. When matrix elements between hadrons with plus momentum $p^{+}$are taken, the lower limit becomes $x^{\prime}=x$, as in (2.10).
} 
for a DPD in a right moving proton, where the short-distance coefficients are defined as

$$
{ }^{R} C_{a b}\left(x, \boldsymbol{z} ; \mu, Y_{C}\right)=\lim _{Y_{L} \rightarrow-\infty} \frac{{ }^{R} C_{\mathrm{us}, a b}\left(x, \boldsymbol{z} ; \mu, Y_{L}\right)}{\sqrt{{ }^{R} C_{S, a}\left(\boldsymbol{z} ; \mu, 2 Y_{C}-2 Y_{L}\right)}} .
$$

Switching variables from $Y_{C}$ to $\zeta$ and again using the definition (4.23) of DPDs, we obtain the final form of the matching equation:

$$
\begin{aligned}
{ }^{R} F_{a_{1} a_{2}}\left(x_{i}, \boldsymbol{z}_{i}, \boldsymbol{y} ; \mu_{i}, \zeta\right)=\sum_{b_{1}, b_{2}}{ }^{R} C_{a_{1} b_{1}}\left(x_{1}^{\prime}, \boldsymbol{z}_{1} ; \mu_{1}, x_{1} \zeta / x_{2}\right) \\
\\
\qquad \stackrel{\otimes}{1}^{\otimes} C_{a_{2} b_{2}}\left(x_{2}^{\prime}, \boldsymbol{z}_{2} ; \mu_{2}, x_{2} \zeta / x_{1}\right) \underset{x_{2}}{\otimes}{ }^{R} F_{b_{1} b_{2}}\left(x_{i}^{\prime}, \boldsymbol{y} ; \mu_{i}, \zeta\right) .
\end{aligned}
$$

The rescaling factors $x_{1} / x_{2}$ or $x_{2} / x_{1}$ of $\zeta$ in the short-distance coefficients arise for the same reason as discussed after (5.8). Note that they involve the parton momentum fractions $x_{1}$ or $x_{2}$ and not the integration variables $x_{1}^{\prime}$ or $x_{2}^{\prime}$ of the convolution (2.10).

Let us emphasise that (6.11) involves mixing between quark and gluon distributions. Therefore, the combination of ${ }^{R R} s(\boldsymbol{y})$ and ${ }^{R} F_{\mathrm{us}, b_{1} b_{2}}\left(x_{i}, \boldsymbol{y}\right)$ into a DPDF works only because ${ }^{R R} S(\boldsymbol{y})$ is the same for quarks and gluons (for representations $R$ accessible to quarks).

Let us finally set $R=1$ and show that in the colour singlet channel, the coefficient ${ }^{1} C_{a b}(x, \boldsymbol{z})$ in $(6.11)$ is identical to the coefficient $C_{a b}(x, \boldsymbol{z})$ in the small- $\boldsymbol{z}$ expansion (2.9) of a single parton TMD. The corresponding identity for the coefficient ${ }^{1} C_{\mathrm{us}, a b}(x, \boldsymbol{z})$ readily follows from taking the matrix element of the operator relation (6.6) between two proton states, which is related to the unsubtracted TMD $f_{\mathrm{us}, a}$ via the first relation in (3.11). For ${ }^{1} C_{S, a}(\boldsymbol{z})$ the identity is obtained by taking the vacuum expectation value of (6.5), which gives the soft factor $S_{a}(\boldsymbol{z})$ relevant for constructing the TMD $f_{a}(x, \boldsymbol{z})$ via (3.47) and (3.49). The short-distance coefficient for $f_{a}(x, \boldsymbol{z})$ is then obtained as in (6.10).

Notice that the vacuum expectation value of the colour projected operator on the r.h.s. of (6.5) gives unity for $R=1$, because the Wilson lines along both $v_{L}$ and $v_{R}$ appear in the combination $W W^{\dagger}=\mathbb{1}$. This reflects the fact that no soft factor appears in the definition of ordinary PDFs. We thus find

$$
S_{a}(\boldsymbol{z} ; \mu, Y)={ }^{1} C_{S, a}(\boldsymbol{z} ; \mu, Y)
$$

for small $\boldsymbol{z}$, i.e. the soft factor for single TMD factorisation is purely perturbative at small distances.

\subsubsection{Evolution equations and their solution}

Let us now establish the consequences of the short-distance expansion (6.11) for scale and rapidity evolution. From (6.8) we deduce that

$$
\begin{aligned}
& \frac{\partial}{\partial Y} \log \sqrt{{ }^{R} C_{S, a_{1}}\left(\boldsymbol{z}_{1} ; \mu_{1}, 2 Y\right)}+\frac{\partial}{\partial Y} \log \sqrt{{ }^{R} C_{S, a_{2}}\left(\boldsymbol{z}_{2} ; \mu_{2}, 2 Y\right)} \\
& =\frac{\partial}{\partial Y} \log R R_{s_{a_{1} a_{2}}}\left(\boldsymbol{z}_{i}, \boldsymbol{y} ; \mu_{i}, Y\right)-\frac{\partial}{\partial Y} \log R R_{s}\left(\boldsymbol{y} ; \mu_{i}, Y\right) \\
& ={ }^{R R} K_{a_{1} a_{2}}\left(\boldsymbol{z}_{i}, \boldsymbol{y} ; \mu_{i}\right)-{ }^{R} J\left(\boldsymbol{y} ; \mu_{i}\right)
\end{aligned}
$$


with the kernels ${ }^{R R} K_{a_{1} a_{2}}$ and ${ }^{R} J$ from (3.24) and (5.22). The rapidity derivative of ${ }^{R} C_{S, a}(\boldsymbol{z} ; \mu, 2 Y)$ must thus be independent of $Y$. Defining

$$
{ }^{R} K_{a}(\boldsymbol{z} ; \mu)=\frac{\partial}{\partial Y} \log { }^{R} C_{S, a}(\boldsymbol{z} ; \mu, Y),
$$

we obtain

$$
\frac{\partial}{\partial \log \zeta}{ }^{R} C_{a b}(x, \boldsymbol{z} ; \mu, \zeta)=\frac{1}{2}{ }^{R} K_{a}(\boldsymbol{z} ; \mu){ }^{R} C_{a b}(x, \boldsymbol{z} ; \mu, \zeta)
$$

for the coefficients in (6.10). Inserting this into (6.11), we find

$$
{ }^{R R^{\prime}} K_{a_{1} a_{2}}\left(\boldsymbol{z}_{i}, \boldsymbol{y} ; \mu_{i}\right)=\delta_{R R^{\prime}}\left[{ }^{R} K_{a_{1}}\left(\boldsymbol{z}_{1} ; \mu_{1}\right)+{ }^{R} K_{a_{2}}\left(\boldsymbol{z}_{2} ; \mu_{2}\right)+{ }^{R} J\left(\boldsymbol{y} ; \mu_{i}\right)\right] .
$$

This represents a significant simplification of the Collins-Soper equation for DTMDs in the small $\boldsymbol{z}_{i}$ limit, both in the colour structure and in the separation of the three distance variables.

Taking the derivative $\partial / \partial \log \mu_{1}$ of (6.16) and using the renormalisation group equations (5.12) and (5.23) for ${ }^{R} R^{\prime} K_{a_{1} a_{2}}$ and ${ }^{R} J$, we deduce that

$$
\gamma_{K, a}(\mu)={ }^{R} \gamma_{K, a}(\mu)+{ }^{R} \gamma_{J}(\mu)
$$

for all $R$, where we have introduced the anomalous dimension of the kernel ${ }^{R} K_{a}$,

$$
\frac{\partial}{\partial \log \mu}{ }^{R} K_{a}(\boldsymbol{z} ; \mu)=-{ }^{R} \gamma_{K, a}(\mu) .
$$

In the colour singlet sector, we have ${ }^{1} J=0$ and hence ${ }^{1} \gamma_{J}=0$, which implies ${ }^{1} \gamma_{K, a}=\gamma_{K, a}$. Indeed, ${ }^{1} K_{a}(\boldsymbol{z} ; \mu)$ is identical to the ordinary Collins-Soper kernel $K_{a}(\boldsymbol{z} ; \mu)$ in $(2.7)$, which is readily shown by taking the rapidity derivative of (6.12).

Taking the $\mu_{1}$ derivative of the short-distance expansion (6.11) and using the evolution equations (5.7) and (5.24) for DTMDs and DPDFs, we obtain

$$
\begin{aligned}
& \frac{\partial}{\partial \log \mu}{ }^{R} C_{a c}(x, \boldsymbol{z} ; \mu, \zeta) \\
& \quad=\sum_{b}{ }^{R} C_{a b}\left(x^{\prime}, \boldsymbol{z} ; \mu, \zeta\right) \underset{x}{\otimes}\left[\delta_{b c} \delta\left(1-x^{\prime}\right) \gamma_{F, c}(\mu, \zeta)-2{ }^{R} P_{b c}\left(x^{\prime} ; \mu, \zeta\right)\right] .
\end{aligned}
$$

Having computed the short-distance kernel ${ }^{R} C_{a b}$ at a certain order in $\alpha_{s}$, we can use this relation to reconstruct the evolution kernel ${ }^{R} P_{a b}$ at the same order. We will do this in section 7.3.5.

With the short-distance limit (6.16) of the Collins-Soper kernel, the general solution (5.17) of the evolution equations for DTMDs takes the form

$$
\begin{aligned}
& { }^{R} F_{a_{1} a_{2}}\left(x_{i}, \boldsymbol{z}_{i}, \boldsymbol{y} ; \mu_{i}, \zeta\right) \\
& =\exp \left\{\int_{\mu_{01}}^{\mu_{1}} \frac{d \mu}{\mu}\left[\gamma_{a_{1}}(\mu)-\gamma_{K, a_{1}}(\mu) \log \frac{\sqrt{x_{1} \zeta / x_{2}}}{\mu}\right]\right. \\
& \quad+\int_{\mu_{02}}^{\mu_{2}} \frac{d \mu}{\mu}\left[\gamma_{a_{2}}(\mu)-\gamma_{K, a_{2}}(\mu) \log \frac{\sqrt{x_{2} \zeta / x_{1}}}{\mu}\right] \\
& \left.\quad+\left[{ }^{R} K_{a_{1}}\left(\boldsymbol{z}_{1} ; \mu_{01}\right)+{ }^{R} K_{a_{2}}\left(\boldsymbol{z}_{2} ; \mu_{02}\right)+{ }^{R} J\left(\boldsymbol{y} ; \mu_{0 i}\right)\right] \log \frac{\sqrt{\zeta}}{\sqrt{\zeta_{0}}}\right\}{ }^{R} F_{a_{1} a_{2}}\left(x_{i}, \boldsymbol{z}_{i}, \boldsymbol{y} ; \mu_{0 i}, \zeta_{0}\right),
\end{aligned}
$$


where different colour channels no longer mix with each other. Using (6.15), we can rewrite the rapidity dependence of the short-distance kernels as

$$
{ }^{R} C_{a_{1} b_{1}}\left(x_{1}^{\prime}, \boldsymbol{z}_{1} ; \mu_{01}, x_{1} \zeta_{0} / x_{2}\right)=\exp \left[{ }^{R} K_{a_{1}}\left(\boldsymbol{z}_{1} ; \mu_{01}\right) \log \frac{\sqrt{x_{1} \zeta_{0} / x_{2}}}{\mu_{01}}\right]{ }^{R} C_{a_{1} b_{1}}\left(x_{1}^{\prime}, \boldsymbol{z}_{1} ; \mu_{01}, \mu_{01}^{2}\right)
$$

and likewise for the index 2, which reduces the number of independent scales in $C_{a b}$. Using the short-distance matching (6.11) for ${ }^{R} F_{a_{1} a_{2}}\left(x_{i}, \boldsymbol{z}_{i}, \boldsymbol{y} ; \mu_{0 i}, \zeta_{0}\right)$ in (6.20), we obtain our master formula for DTMDs at small $\boldsymbol{z}_{i}$ and large $\boldsymbol{y}$ :

$$
\begin{aligned}
& { }^{R} F_{a_{1} a_{2}}\left(x_{i}, \boldsymbol{z}_{i}, \boldsymbol{y} ; \mu_{i}, \zeta\right) \\
& =\exp \left\{\int_{\mu_{01}}^{\mu_{1}} \frac{d \mu}{\mu}\left[\gamma_{a_{1}}(\mu)-\gamma_{K, a_{1}}(\mu) \log \frac{\sqrt{x_{1} \zeta / x_{2}}}{\mu}\right]+{ }^{R} K_{a_{1}}\left(\boldsymbol{z}_{1} ; \mu_{01}\right) \log \frac{\sqrt{x_{1} \zeta / x_{2}}}{\mu_{01}}\right. \\
& \quad+\int_{\mu_{02}}^{\mu_{2}} \frac{d \mu}{\mu}\left[\gamma_{a_{2}}(\mu)-\gamma_{K, a_{2}}(\mu) \log \frac{\sqrt{x_{2} \zeta / x_{1}}}{\mu}\right]+{ }^{R} K_{a_{2}}\left(\boldsymbol{z}_{2} ; \mu_{02}\right) \log \frac{\sqrt{x_{2} \zeta / x_{1}}}{\mu_{02}} \\
& \left.\quad+{ }^{R} J\left(\boldsymbol{y} ; \mu_{0 i}\right) \log \frac{\sqrt{\zeta}}{\sqrt{\zeta_{0}}}\right\} \\
& \quad \times \sum_{b_{1}, b_{2}}{ }^{R} C_{a_{1} b_{1}}\left(x_{1}^{\prime}, \boldsymbol{z}_{1} ; \mu_{01}, \mu_{01}^{2}\right) \underset{x_{1}}{\otimes} C_{a_{2} b_{2}}\left(x_{2}^{\prime}, \boldsymbol{z}_{2} ; \mu_{02}, \mu_{02}^{2}\right) \underset{x_{2}}{\otimes}{ }^{R} F_{b_{1} b_{2}}\left(x_{i}^{\prime}, \boldsymbol{y} ; \mu_{0 i}, \zeta_{0}\right) .
\end{aligned}
$$

In the colour singlet channel, the result (6.22) is a simple copy of its analogue (2.11) for a single-parton TMD, with separate short-distance coefficients $C_{a b}$ and Sudakov exponentials for each parton. For colour non-singlet channels, the Collins-Soper kernels ${ }^{R} K_{a}(\boldsymbol{z})$ acquire a colour dependence, and there is an additional term ${ }^{R} J(\boldsymbol{y})$ in the exponent. The latter is also present in collinear DPS factorisation: in fact one simply has

$$
\exp \left[{ }^{R} J\left(\boldsymbol{y} ; \mu_{0 i}\right) \log \frac{\sqrt{\zeta}}{\sqrt{\zeta_{0}}}\right]{ }^{R} F_{b_{1} b_{2}}\left(x_{i}^{\prime}, \boldsymbol{y} ; \mu_{0 i}, \zeta_{0}\right)={ }^{R} F_{b_{1} b_{2}}\left(x_{i}^{\prime}, \boldsymbol{y} ; \mu_{0 i}, \zeta\right)
$$

according to (5.22). In this sense, the value of $\zeta_{0}$ in (6.22) is irrelevant (contrary to the choice of $\mu_{0 i}$, which appears in quantities that are computed using fixed-order perturbation theory). The choice of $\zeta_{0}$ does matter when this is the scale at which one formulates an ansatz or model for the DPDFs (which is of course inevitable for concrete calculations).

After inserting (6.22) and its counterpart for the left moving proton into the definition (5.2) of $W$, we can combine the logarithms of $\zeta$ and $\bar{\zeta}$ in the exponentials, as we did 
in the generic expression (5.19). We thus obtain

$$
\begin{aligned}
& W_{\text {large } y}=\sum_{R} \eta_{a_{1} a_{2}}(R) \\
& \times \exp \left\{\int_{\mu_{01}}^{\mu_{1}} \frac{d \mu}{\mu}\left[\gamma_{a_{1}}(\mu)-\gamma_{K, a_{1}}(\mu) \log \frac{Q_{1}^{2}}{\mu^{2}}\right]+{ }^{R} K_{a_{1}}\left(\boldsymbol{z}_{1} ; \mu_{01}\right) \log \frac{Q_{1}^{2}}{\mu_{01}^{2}}\right. \\
& \left.\quad+\int_{\mu_{02}}^{\mu_{2}} \frac{d \mu}{\mu}\left[\gamma_{a_{2}}(\mu)-\gamma_{K, a_{2}}(\mu) \log \frac{Q_{2}^{2}}{\mu^{2}}\right]+{ }^{R} K_{a_{2}}\left(\boldsymbol{z}_{2} ; \mu_{02}\right) \log \frac{Q_{2}^{2}}{\mu_{02}^{2}}\right\} \\
& \times \sum_{c_{1}, c_{2}, d_{1}, d_{2}}{ }^{R} C_{b_{1} d_{1}}\left(\bar{x}_{1}^{\prime}, \boldsymbol{z}_{1} ; \mu_{01}, \mu_{01}^{2}\right) \underset{\bar{x}_{1}}{\otimes}{ }^{R} C_{b_{2} d_{2}}\left(\bar{x}_{2}^{\prime}, \boldsymbol{z}_{2} ; \mu_{02}, \mu_{02}^{2}\right) \\
& \quad \stackrel{\otimes}{{ }^{R}} C_{a_{1} c_{1}}\left(x_{1}^{\prime}, \boldsymbol{z}_{1} ; \mu_{01}, \mu_{01}^{2}\right) \underset{x_{1}}{\otimes}{ }^{R} C_{a_{2} c_{2}}\left(x_{2}^{\prime}, \boldsymbol{z}_{2} ; \mu_{02}, \mu_{02}^{2}\right) \\
& \underset{x_{2}}{\otimes}[\Phi(\nu \boldsymbol{y})]^{2} \exp \left[{ }^{R} J\left(\boldsymbol{y} ; \mu_{0 i}\right) \log \frac{Q_{1} Q_{2}}{\zeta_{0}}\right]{ }^{R} F_{d_{1} d_{2}}\left(\bar{x}_{i}^{\prime}, \boldsymbol{y} ; \mu_{0 i}, \zeta_{0}\right){ }^{R} F_{c_{1} c_{2}}\left(x_{i}^{\prime}, \boldsymbol{y} ; \mu_{0 i}, \zeta_{0}\right),
\end{aligned}
$$

which is the main result of this section. The product of regulator functions $\Phi\left(\nu \boldsymbol{y}_{+}\right) \Phi\left(\nu \boldsymbol{y}_{-}\right)$ has been approximated as appropriate for $\left|\boldsymbol{z}_{1}\right|,\left|\boldsymbol{z}_{2}\right| \ll|\boldsymbol{y}|$. Note that the dependence on $\boldsymbol{y}$, $\boldsymbol{z}_{1}$ and $\boldsymbol{z}_{2}$ is completely factorised in (6.24). The $\boldsymbol{y}$ integral can hence be performed separately. For unpolarised or longitudinally polarised partons, the short-distance coefficients $C$ are independent of the direction of $\boldsymbol{z}_{1}, \boldsymbol{z}_{2}$ by rotation invariance. The angular part of the $\boldsymbol{z}_{i}$ integrations can then readily be performed and turns the Fourier exponentials $e^{-i \boldsymbol{q}_{i} \boldsymbol{z}_{i}}$ into Bessel functions. The situation for transverse quark or linear gluon polarisation, where $C$ (as well as $F$ and $\hat{\sigma}$ ) carries transverse indices, can be discussed along similar lines.

Each of the two partonic cross sections $\hat{\sigma}_{i}$ in (5.1) and the four short-distance kernels ${ }^{R} C$ in (6.24) has an $\alpha_{s}$ expansion starting at order $\alpha_{s}^{0}$. It is natural (although not mandatory) to truncate their product to the highest order in $\alpha_{s}$ at which the individual factors are computed.

To evaluate the kernels ${ }^{R} K$ and ${ }^{R} C$ in (6.24) in fixed-order perturbation theory, one should choose the scales $\mu_{0 i}$ such that no large logarithms appear at higher orders. A standard choice for single hard scattering is to take $\mu_{0 i}^{2}=b_{0}^{2} / z_{i}^{2}$, which makes the one-loop expression of ${ }^{R} K\left(\boldsymbol{z}_{i} ; \mu_{0 i}\right)$ vanish. Here

$$
b_{0}=2 e^{-\gamma_{E}}
$$

with $\gamma_{E}$ being the Euler constant. While the cross section (5.1) is dominated by $\left|\boldsymbol{z}_{i}\right| \sim 1 /\left|\boldsymbol{q}_{i}\right|$ due to the Fourier exponentials $e^{-i \boldsymbol{q}_{i} \boldsymbol{z}_{i}}$, one still must integrate over the full range of these distances. To avoid evaluating the DPDs at unreasonably small factorisation scales $\mu_{0 i}$, one may modify the above scale choice and take instead $\mu_{0 i}=\mu_{z_{i}}$, where for any transverse distance vector $\boldsymbol{b}$ we define

$$
\mu_{b}^{2}=\frac{b_{0}^{2}}{\boldsymbol{b}^{* 2}}, \quad \text { with } \quad \boldsymbol{b}^{*}(\boldsymbol{b}) \underset{\boldsymbol{b} \rightarrow \mathbf{0}}{\longrightarrow} \boldsymbol{b} \text { and } \quad\left|\boldsymbol{b}^{*}(\boldsymbol{b})\right| \underset{|\boldsymbol{b}| \rightarrow \infty}{\longrightarrow} b_{\max }
$$


where $b_{\max }$ is chosen such that $\boldsymbol{b}^{*}$ remains in the region where one trusts perturbative theory even when $\boldsymbol{b}$ becomes large. A possible choice for $\boldsymbol{b}^{*}$ is

$$
\boldsymbol{b}^{*}=\frac{\boldsymbol{b}}{\sqrt{1+\boldsymbol{b}^{2} / b_{\max }^{2}}},
$$

which was proposed long ago $[57,72]$ and is extensively used in TMD phenomenology, but other functional forms have also been explored [93]. We note that it is not mandatory to take factorisation scales proportional to inverse distances, referring to [61, 94] and [85, 95] for examples of scale setting in momentum space and to section 7.1.2 of [96] for a brief synopsis. Most results in our paper do not depend on choosing scales as in (6.26). An exception is the discussion of scale setting in section 6.4 and appendix $\mathrm{C}$, which needs to be adapted if a different choice is made.

In the colour nonsinglet sector, the DPDs ${ }^{R} F$ in (6.24) still involve the different scales $\mu_{0 i}$ and $\zeta_{0}$. To disentangle this dependence further, one may use (5.27) and (6.23) to rewrite ${ }^{R} F$ in terms of the distribution ${ }^{R} \widehat{F}$, whose DGLAP evolution does not involve a separate rapidity scale, and which is therefore better suited to make the separation of scales explicit. At the cross section level, one can then replace the last line of (6.24) by

$$
\begin{gathered}
{[\Phi(\nu \boldsymbol{y})]^{2} \exp \left[-\int_{\mu_{0}}^{\mu_{01}} \frac{d \mu}{\mu} R_{\gamma_{J}}(\mu) \log \frac{Q_{1}^{2}}{\mu^{2}}-\int_{\mu_{0}}^{\mu_{02}} \frac{d \mu}{\mu} R_{\gamma_{J}}(\mu) \log \frac{Q_{2}^{2}}{\mu^{2}}\right.} \\
\left.+{ }^{R} J\left(\boldsymbol{y} ; \mu_{0}, \mu_{0}\right) \log \frac{Q_{1} Q_{2}}{\zeta_{0}}\right] R \widehat{F}_{d_{1} d_{2}, \mu_{0}, \zeta_{0}}\left(\bar{x}_{i}^{\prime}, \boldsymbol{y} ; \mu_{0 i}\right){ }^{R} \widehat{F}_{c_{1} c_{2}, \mu_{0}, \zeta_{0}}\left(x_{i}^{\prime}, \boldsymbol{y} ; \mu_{0 i}\right),
\end{gathered}
$$

where we have explicitly indicated that the two renormalisation scales in ${ }^{R} J$ are taken equal. It is natural to take the starting scale of evolution as $\mu_{0}=\sqrt{\zeta_{0}}$. A particular choice is $\mu_{0}=\mu_{y}$, which for large $\boldsymbol{y}$ saturates at a hadronic scale $\mu_{0}=b_{0} / b_{\max }$. This choice ensures that the initial conditions for DPD evolution do not involve widely different scales as $\boldsymbol{y}$ becomes small.

The factor ${ }^{R} J\left(\boldsymbol{y} ; \mu_{0 i}\right)$ in (6.24) is a nonperturbative function in the large- $\boldsymbol{y}$ region, but we can ensure that it has the correct perturbative small-y behaviour by copying part of the so-called $b^{*}$ trick formulated for ordinary TMDs in $[57,72]$. We thus write

$$
{ }^{R} J\left(\boldsymbol{y} ; \mu_{01}, \mu_{02}\right)=-{ }^{R} g_{J}(\boldsymbol{y})+{ }^{R} J\left(\boldsymbol{y}^{*} ; \mu_{y}, \mu_{y}\right)-\int_{\mu_{y}}^{\mu_{01}} \frac{d \mu}{\mu} R_{\gamma_{J}}(\mu)-\int_{\mu_{y}}^{\mu_{02}} \frac{d \mu}{\mu} R_{\gamma_{J}}(\mu),
$$

where the nonperturbative information is contained in

$$
{ }^{R} g_{J}(\boldsymbol{y})={ }^{R} J\left(\boldsymbol{y}^{*} ; \mu_{i}\right)-{ }^{R} J\left(\boldsymbol{y} ; \mu_{i}\right) .
$$

Because of (5.23), the dependence on the renormalisation scales drops out in ${ }^{R} g_{J}$, which satisfies ${ }^{R} g_{J}(\mathbf{0})=0$ by construction. The term ${ }^{R} J\left(\boldsymbol{y}^{*} ; \mu_{y}, \mu_{y}\right)$ in (6.28) can be evaluated in fixed-order perturbation theory; according to the results in section 7.2 it is zero up to terms of $\mathcal{O}\left(\alpha_{s}^{2}\right)$. Of course, the form (6.28) with $\mu_{0 i}$ replaced by $\mu_{0}$ can be used in (6.27).

In the colour octet sector, the relation (4.49) implies that

$$
{ }^{8} J(\boldsymbol{y} ; \mu, \mu)=K_{g}(\boldsymbol{y} ; \mu),
$$


where $K_{g}$ is the Collins Soper kernel for single gluon TMDs. While little is known about this kernel for nonperturbative distances $\boldsymbol{y}$, one may construct a model by connecting it with its counterpart $K_{q}$ in the quark-antiquark channel, for which there is a considerable body of phenomenology from single Drell-Yan production and from semi-inclusive DIS, see e.g. $[97,98]$ and references therein. A simple possibility would be to assume Casimir scaling, $K_{g} / C_{A}=K_{q} / C_{F}$. This scaling holds perturbatively up to $\mathcal{O}\left(\alpha_{s}^{3}\right)$, as can for instance be seen in appendix D of [99]. ${ }^{10}$

\subsubsection{Extrapolation to large $z_{1}$ and $z_{2}$}

If $\boldsymbol{q}_{1}$ and $\boldsymbol{q}_{2}$ are not sufficiently large to ensure dominance of small $\boldsymbol{z}_{i}$ in the TMD cross section, then a more realistic description of the integrand for large $\boldsymbol{z}_{i}$ is necessary. Of course, a corresponding statement holds already for single hard scattering. A widely used procedure in that case is the $b^{*}$ trick of $[57,72]$. Let us briefly show how it can be adapted to DPS. We recall the solution (5.14) of the rapidity evolution equation,

$$
{ }^{R} F_{a_{1} a_{2}}\left(x_{i}, \boldsymbol{z}_{i}, \boldsymbol{y} ; \mu_{i}, \zeta\right)=\sum_{R^{\prime}} R R^{\prime} \exp \left[K_{a_{1} a_{2}}\left(\boldsymbol{z}_{i}, \boldsymbol{y} ; \mu_{i}\right) \log \frac{\sqrt{\zeta}}{\sqrt{\zeta_{0}}}\right] R^{\prime} F_{a_{1} a_{2}}\left(x_{i}, \boldsymbol{z}_{i}, \boldsymbol{y} ; \mu_{i}, \zeta_{0}\right)
$$

and introduce

$$
\begin{aligned}
R_{g_{F, a_{1} a_{2}}}\left(x_{i}, \boldsymbol{z}_{i}, \boldsymbol{y} ; \zeta\right) & =\log \frac{{ }^{R} F_{a_{1} a_{2}}\left(x_{i}, \boldsymbol{z}_{i}^{*}, \boldsymbol{y} ; \mu_{i}, \zeta\right)}{R F_{a_{1} a_{2}}\left(x_{i}, \boldsymbol{z}_{i}, \boldsymbol{y} ; \mu_{i}, \zeta\right)} \\
R R^{\prime} g_{K, a_{1} a_{2}}\left(\boldsymbol{z}_{i}, \boldsymbol{y}\right) & ={ }^{R} R^{\prime} K_{a_{1} a_{2}}\left(\boldsymbol{z}_{i}^{*}, \boldsymbol{y} ; \mu_{i}\right)-{ }^{R R^{\prime}} K_{a_{1} a_{2}}\left(\boldsymbol{z}_{i}, \boldsymbol{y} ; \mu_{i}\right)
\end{aligned}
$$

with $\boldsymbol{z}^{*}$ defined by (6.26). Both $g_{F}$ and $g_{K}$ are independent of $\mu_{i}$ according to (5.7) and (5.12), and both functions depend on the parameter $b_{\max }$ via $\boldsymbol{z}_{i}^{*}$. By construction, both functions vanish at the point $\boldsymbol{z}_{1}=\boldsymbol{z}_{2}=\mathbf{0}$. We can then write

$$
\begin{aligned}
{ }^{R} F_{a_{1} a_{2}}\left(x_{i}, \boldsymbol{z}_{i}, \boldsymbol{y} ; \mu_{i}, \zeta\right)= & \sum_{R^{\prime}} R R^{\prime} \exp \left[-g_{K, a_{1} a_{2}}\left(\boldsymbol{z}_{i}, \boldsymbol{y}\right) \log \frac{\sqrt{\zeta}}{\sqrt{\zeta_{0}}}+K_{a_{1} a_{2}}\left(\boldsymbol{z}_{i}^{*}, \boldsymbol{y} ; \mu_{i}\right) \log \frac{\sqrt{\zeta}}{\sqrt{\zeta_{0}}}\right] \\
& \times \exp \left[-{ }^{R^{\prime}} g_{F, a_{1} a_{2}}\left(x_{i}, \boldsymbol{z}_{i}, \boldsymbol{y} ; \zeta_{0}\right)\right]{ }^{R^{\prime}} F_{a_{1} a_{2}}\left(x_{i}, \boldsymbol{z}_{i}^{*}, \boldsymbol{y} ; \mu_{i}, \zeta_{0}\right) .
\end{aligned}
$$

In this expression, ${ }^{R R^{\prime}} K_{a_{1} a_{2}}$ and ${ }^{R^{\prime}} F_{a_{1} a_{2}}$ can be evaluated using the short-distance expansion discussed in the previous subsection, provided that $\left|z_{1}^{*}\right|,\left|z_{2}^{*}\right| \ll|\boldsymbol{y}|$. As a consequence, $R R^{\prime} K_{a_{1} a_{2}}$ is diagonal in $R$ and $R^{\prime}$. By contrast, the functions $R R^{\prime} g_{K, a_{1} a_{2}}$ and ${ }^{R} g_{F, a_{1} a_{2}}$ are entirely nonperturbative and need to be modelled. This is a daunting task, because they depend on several transverse variables, there are many functions in the different colour channels and there is less guidance from data. Using the $b^{*}$ trick for computing DPS processes with $\boldsymbol{q}_{1}$ and $\boldsymbol{q}_{2}$ in the nonperturbative region would therefore require additional theory input, or strong simplifying assumptions.

The $b^{*}$ trick is not the only way to handle the region of large transverse distances, and for TMD factorisation in SPS a variety of other methods have been employed [61, 85,

\footnotetext{
${ }^{10}$ Just recently it has been found [100] that Casimir scaling for the cusp anomalous dimension, $\gamma_{K, g} / C_{A}=$ $\gamma_{K, q} / C_{F}$, is broken at $\mathcal{O}\left(\alpha_{s}^{4}\right)$. Equation (2.8) implies the breaking of Casimir scaling for $K_{a}$ at the same order. For a related calculation see [101].
} 
94, 95, 102-107]. It would be interesting to study if and how they could be adapted to DPS. This is however beyond the scope of the present work, where we focus on transverse momenta in the perturbative region.

\subsection{The small- $y$ region}

We now consider the region where $|\boldsymbol{y}|$ is of the same order as $\left|\boldsymbol{z}_{1}\right|$ and $\left|\boldsymbol{z}_{2}\right|$, with all distances being small compared with $1 / \Lambda$. Again, we will derive double parton analogues of the relations (2.9) and (2.11) between TMDs and PDFs. They are given in (6.38), (6.39) and (6.44) and involve collinear PDFs and twist-four distributions as nonperturbative input, which also appear in the expansion (6.42) of DPDFs at small $\boldsymbol{y}$. At the cross section level, we then obtain the expression (6.47) in which all large logarithms are explicitly resummed. An important finding is that the different combinations of collinear PDFs and twist-four distributions have different power behaviour in the cross section, specified in (6.40) and compared with the contribution from large $\boldsymbol{y}$ in (6.41).

\subsubsection{Short-distance expansion}

Let us first establish that the soft factor, and hence the Collins-Soper kernel ${ }^{R} R^{\prime} K_{a_{1} a_{2}}$, can be fully computed in perturbation theory when all transverse distances become small. Repeating the arguments that lead to (6.2), we obtain

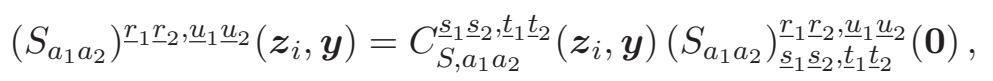

which involves the extended soft factor (3.12) with all distance arguments set to zero and a short-distance coefficient $C_{S, a_{1} a_{2}}$ describing interactions between all Wilson lines. Inserting colour projectors as in (4.12), we get

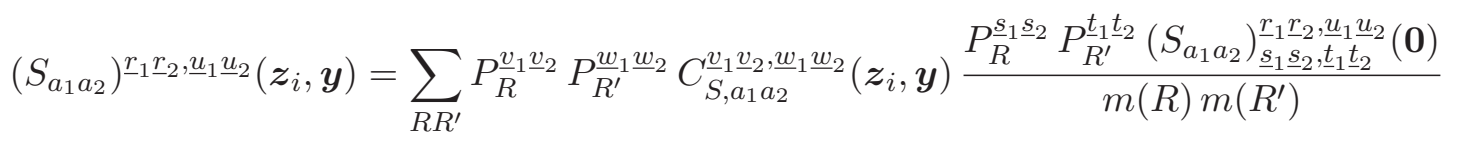

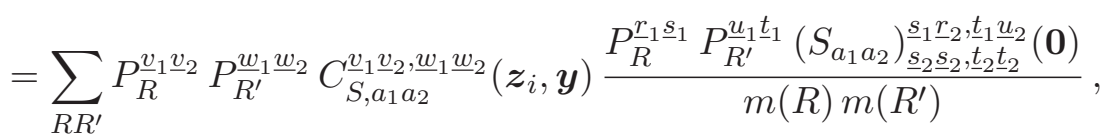

where in the second step the identity (4.41) has been used for the indices associated with parton $a_{1}$ in $S_{a_{1} a_{2}}$. The sum over $\underline{s}_{2}$ on the r.h.s. now ties together pairs of conjugate Wilson lines along the same direction and at the same transverse position, which results in unit matrices according to $W(\boldsymbol{\xi}, v) W^{\dagger}(\boldsymbol{\xi}, v)=\mathbb{1}$. For $S_{q q}$ this is represented pictorially in figure 8 . The same holds for the sum over $\underline{t}_{2}$. The extended soft factor thus collapses to a product of unit matrices, and we have

$$
\left(S_{a_{1} a_{2}}\right)^{\underline{r}_{1} \underline{r}_{2}, \underline{u}_{1} \underline{u}_{2}}\left(\boldsymbol{z}_{i}, \boldsymbol{y}\right)=\sum_{R R^{\prime}} P_{R}^{\underline{v}_{1} \underline{v}_{2}} P_{R^{\prime}}^{\underline{w}_{1}} \underline{w}_{2} C_{S, a_{1} a_{2}}^{\underline{v}_{1} \underline{v}_{2}, \underline{w}_{1} \underline{w}_{2}}\left(\boldsymbol{z}_{i}, \boldsymbol{y}\right) \frac{P_{R}^{\underline{r}_{1}} \underline{\underline{r}}_{2} P_{R^{\prime}}^{\underline{u}_{1}} \underline{u}_{2}}{m(R) m\left(R^{\prime}\right)} .
$$

Projecting this on definite representations for $\left(\underline{r}_{1} \underline{r}_{2}\right)$ and for $\left(\underline{u}_{1} \underline{u}_{2}\right)$, we simply obtain

$$
{ }^{R R^{\prime}} S_{a_{1} a_{2}}\left(\boldsymbol{z}_{i}, \boldsymbol{y} ; \mu_{i}, Y\right)={ }^{R R^{\prime}} C_{S, a_{1} a_{2}}\left(\boldsymbol{z}_{i}, \boldsymbol{y} ; \mu_{i}, Y\right),
$$




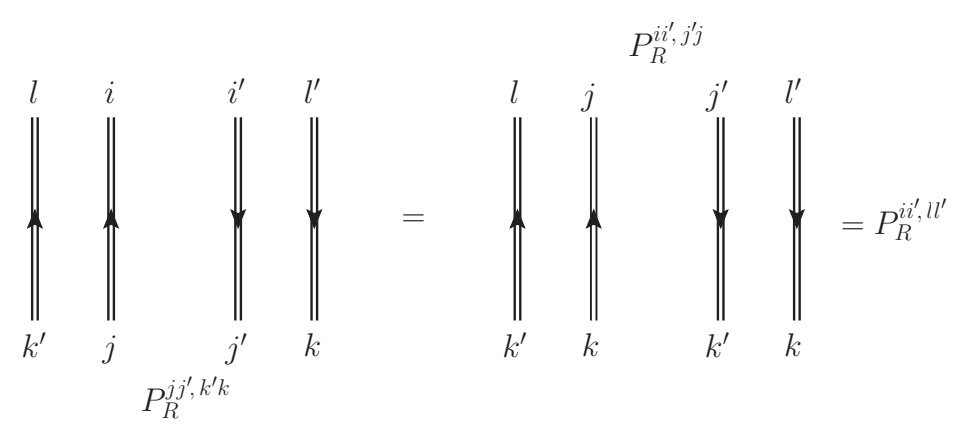

Figure 8. Simplification in the extended soft factor $S_{q q}$ with all Wilson lines at the same transverse position, using the identity in figure 2 and the unitarity of Wilson lines. Analogous relations hold for indices in the adjoint colour representation.

where the projection ${ }^{R} R^{\prime} C$ is defined in analogy to (4.20) and where we have restored all arguments. In analogy to the case (6.12) for SPS, we thus find that when all transverse distances become small, the soft factor for DPS is equal to its matching coefficient and thus can be entirely computed in perturbation theory. The same then holds for the CollinsSoper kernel ${ }^{R R^{\prime}} K$ and for the matrix $R R^{\prime}$, which can be constructed from ${ }^{R R^{\prime}} S$ as shown in appendix A. An explicit check of this result is the fact that the one-loop expression for $R R^{\prime} K$ in section 7.2 is free of infrared divergences, as is the two-loop soft factor for DPS computed in [74].

We now turn to the short-distance limit of the collinear matrix elements, which can be discussed in close analogy to the small-y limit of collinear DPDs in section 3.3 of [30]. In the limit of small $\boldsymbol{z}_{i}$ and $\boldsymbol{y}$, the unsubtracted distributions ${ }^{R^{\prime}} F_{\text {us }}$ are given by short-distance coefficients times proton matrix elements of operators with all fields at transverse position $\mathbf{0}$. The latter are given by collinear parton distributions of different twist. Multiplying with the perturbative expression of $R^{\prime} s$, one obtains the expansion of ${ }^{R} F$. Adapting the notation in [30], we write ${ }^{11}$

$$
{ }^{R} F={ }^{R} F_{\mathrm{spl}}+{ }^{R} F_{\mathrm{tw} 3}+{ }^{R} F_{\mathrm{int}},
$$

where the short-distance expansion of the terms on the r.h.s. involves proton matrix elements of operators with twist two, twist three and twist four, respectively. Following the discussion in section 2.1 of [30], we neglect the contribution with collinear twist-three distributions, which for unpolarised protons are chiral odd. They have no dynamic cross talk with gluon distributions and are therefore expected to be small compared with twist-two and twist-four distributions at small $x$.

The splitting contribution $F_{\mathrm{spl}}$ describes the case where a single parton splits into partons $a_{1}$ and $a_{2}$, as shown in figure $6 \mathrm{a}$ at lowest order in $\alpha_{s}$. It can be obtained from (5.37) by taking the limit of small $\boldsymbol{Z}$. For the $\mathcal{O}\left(\alpha_{s}\right)$ term, this simply corresponds to replacing the TMD $f_{a_{0}}\left(x_{1}+x_{2}, \boldsymbol{Z}\right)$ by the PDF $f_{a_{0}}\left(x_{1}+x_{2}\right)$. Since the proton is unpolarised, the

\footnotetext{
${ }^{11}$ The distributions on the r.h.s. of (6.37) are respectively denoted by $F_{\mathrm{spl}, \mathrm{pt}}, F_{\mathrm{int}, \mathrm{pt}}$ and $F_{\mathrm{tw} 3, \mathrm{pt}}$ in [30]. For brevity we omit the specification "pt" here.
} 
parton $a_{0}$ in the PDF is unpolarised as well. We thus obtain

$$
{ }^{R} F_{a_{1} a_{2}, \mathrm{spl}}\left(x_{i}, \boldsymbol{z}_{i}, \boldsymbol{y} ; \mu, \mu, \zeta\right)=\frac{\boldsymbol{y}_{+}^{l} \boldsymbol{y}_{-}^{l^{\prime}}}{\boldsymbol{y}_{+}^{2} \boldsymbol{y}_{-}^{2}} \frac{\alpha_{s}(\mu)}{2 \pi^{2}}{ }^{R} T_{a_{0} \rightarrow a_{1} a_{2}}^{l l^{\prime}}\left(\frac{x_{1}}{x_{1}+x_{2}}\right) \frac{f_{a_{0}}\left(x_{1}+x_{2} ; \mu\right)}{x_{1}+x_{2}}+\mathcal{O}\left(\alpha_{s}^{2}\right),
$$

where we have restored the dependence on the UV renormalisation scale, taken equal for the partons with momentum fraction $x_{1}$ and $x_{2}$. A $\zeta$ dependence appears only at order $\alpha_{s}^{2}$. To avoid large logarithms of the higher-order terms in the region of small $\left|\boldsymbol{z}_{1}\right| \sim\left|\boldsymbol{z}_{2}\right| \sim|\boldsymbol{y}|$, any choice where $1 / \mu$ and $1 / \sqrt{\zeta}$ are of the order of these distances will do. A particular choice is given in $(6.45)$ below.

The term $F_{\text {int }}$ in (6.37) is referred to as the "intrinsic" contribution to the DPD and may be thought of as describing parton pairs $a_{1}, a_{2}$ in the "intrinsic" proton wave function. It is the only contribution starting at order $\alpha_{s}^{0}$ and reads

$$
{ }^{R} F_{a_{1} a_{2}, \text { int }}\left(x_{i}, \boldsymbol{z}_{i}, \boldsymbol{y} ; \mu, \mu, \zeta\right)={ }^{R} G_{a_{1} a_{2}}\left(x_{1}, x_{2}, x_{2}, x_{1} ; \mu\right)+\mathcal{O}\left(\alpha_{s}\right),
$$

where ${ }^{R} G$ denotes a collinear twist-four distribution. The lowest-order term is simply obtained by setting $\boldsymbol{z}_{i}=\boldsymbol{y}=\mathbf{0}$ in the matrix element (3.2) (this must be done before renormalisation and hence in $D=4-2 \epsilon$ dimensions). The $\mathcal{O}\left(\alpha_{s}\right)$ term involves shortdistance interactions and is briefly discussed in section 3.3 of [30].

Inserting (6.37) with (6.38) and (6.39) into the cross section formula (5.1), we can derive the power behaviour of the contribution from the region $\left|\boldsymbol{z}_{1}\right| \sim\left|\boldsymbol{z}_{2}\right| \sim|\boldsymbol{y}| \sim 1 / q_{T}$, using that the twist-four distribution scales like $G \sim \Lambda^{2}$. For the scaled DPS cross section we obtain

$$
\left.Q^{4} \frac{d \sigma_{\text {DPS }}}{d^{2} \boldsymbol{q}_{1} d^{2} \boldsymbol{q}_{2}}\right|_{\text {small } y} \sim\left\{\begin{array}{lll}
\alpha_{s}^{2} / q_{T}^{2} & \text { from } F_{\mathrm{spl}} \times F_{\mathrm{spl}} & (1 \mathrm{v} 1) \\
\alpha_{s} \Lambda^{2} / q_{T}^{4} & \text { from } F_{\mathrm{spl}} \times F_{\mathrm{int}} & (2 \mathrm{v} 1) \\
\Lambda^{4} / q_{T}^{6} & \text { from } F_{\mathrm{int}} \times F_{\mathrm{int}} & (2 \mathrm{v} 2)
\end{array}\right.
$$

where we have also indicated the lowest order in $\alpha_{s}$ for each contribution. For the contribution from large $|\boldsymbol{y}| \sim 1 / \Lambda$ discussed in section 6.2 , we have

$$
\left.Q^{4} \frac{d \sigma_{\mathrm{DPS}}}{d^{2} \boldsymbol{q}_{1} d^{2} \boldsymbol{q}_{2}}\right|_{\text {large } y} \sim \Lambda^{2} / q_{T}^{4}
$$

as follows from the small-distance expansion (6.11) and the power behaviour $F \sim \Lambda^{2}$ of a DPDF at large $\boldsymbol{y}$. We note that (6.40) and (6.41) do not hold in the kinematic region $\left|\boldsymbol{q}_{1}+\boldsymbol{q}_{2}\right| \sim \Lambda$, which we have excluded from our considerations, and which has a different power counting as seen from table 1 in [27]. Notice also that some parton combinations, such as $\left(a_{1} a_{2}\right)=(d \bar{u})$, cannot be obtained by parton splitting at $\mathcal{O}\left(\alpha_{s}\right)$. In this case, $F_{\text {spl }}$ starts at $\mathcal{O}\left(\alpha_{s}^{2}\right)$ and the powers of $\alpha_{s}$ in (6.40) must be adjusted accordingly.

The three contributions in (6.40) are referred to as $1 \mathrm{v} 1,2 \mathrm{v} 1$ and $2 \mathrm{v} 2$ terms as indicated. The 1v1 (read "one versus one") term is associated with graphs as in figure 4a, where one parton in each proton initiates a sequence of short-distance interactions (a splitting at scale $q_{T}$ followed by hard scattering at scale $Q$ ). The $2 \mathrm{v} 2$ term corresponds to graphs where two "intrinsic" partons in each proton directly enter the hard subprocesses, as shown in 


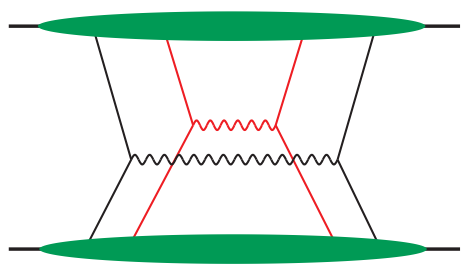

(a)

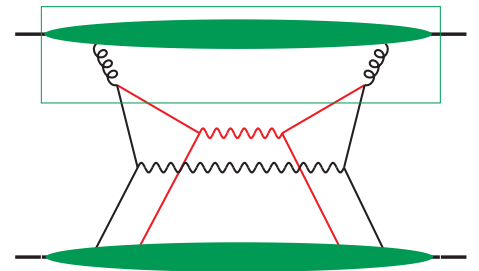

(b)

Figure 9. Counterparts to the $1 \mathrm{v} 1$ graph in figure $4 \mathrm{a}$. In the $2 \mathrm{v} 2$ graph (a) there is no perturbative splitting, and in the $2 \mathrm{v} 1$ graph (b) one has a perturbative splitting in one of the two protons. The box encloses the DTMD of the upper proton and indicates that the splittings take place at transverse distances of order $1 / q_{T}$.

figure 9a. In the $2 \mathrm{v} 1$ term one starts with two "intrinsic" partons in one proton, whereas in the other proton one starts with one parton that splits into two at scale $q_{T}$. This is shown in figure $9 \mathrm{~b}$. It is natural to associate the term in (6.41) with $2 \mathrm{v} 2$ as well, since at large $\boldsymbol{y}$ one cannot identify any perturbative splitting contribution.

Among the terms in (6.40) and (6.41), only the term with $F_{\mathrm{spl}} \times F_{\mathrm{spl}}$ is leading as far as powers of $\Lambda^{2} / q_{T}^{2}$ are concerned. However, the other terms involve fewer powers in $\alpha_{s}$, and they rise more strongly as the momentum fractions $x$ become small, because one roughly expects the intrinsic part of the DPDF to grow like the square of a single PDF in that limit. From this perspective, one may still discard the term $F_{\text {int }} \times F_{\text {int }}$, which is power suppressed compared with the large- $\boldsymbol{y}$ contribution but has the same power in $\alpha_{s}$ and a similar small $x$ behaviour. However, the inclusion of this term in the cross section will in general not require much additional effort, and it renders the combination of the contributions from small and large $\boldsymbol{y}$ more straightforward (see section 6.4).

The expansion of the DPDFs $F\left(x_{i}, \boldsymbol{y}\right)$ for small $\boldsymbol{y}$ proceeds in exactly the same manner. It can be obtained from (6.38) and (6.39) by setting $\boldsymbol{z}_{1}=\boldsymbol{z}_{2}=\mathbf{0}$ before performing UV renormalisation and setting $D=4$. This gives

$$
\begin{aligned}
& { }^{R} F_{a_{1} a_{2}, \text { spl }}\left(x_{i}, \boldsymbol{y} ; \mu, \mu, \zeta\right)=\frac{\boldsymbol{y}^{l} \boldsymbol{y}^{l^{\prime}}}{\boldsymbol{y}^{4}} \frac{\alpha_{s}(\mu)}{2 \pi^{2}}{ }^{R} T_{a_{0} \rightarrow a_{1} a_{2}}^{l l^{\prime}}\left(\frac{x_{1}}{x_{1}+x_{2}}\right) \frac{f_{a_{0}}\left(x_{1}+x_{2} ; \mu\right)}{x_{1}+x_{2}}+\mathcal{O}\left(\alpha_{s}^{2}\right), \\
& { }^{R} F_{a_{1} a_{2}, \text { int }}\left(x_{i}, \boldsymbol{y} ; \mu, \mu, \zeta\right)={ }^{R} G_{a_{1} a_{2}}\left(x_{1}, x_{2}, x_{2}, x_{1} ; \mu\right)+\mathcal{O}\left(\alpha_{s}\right),
\end{aligned}
$$

where for $F_{\text {int }}$ the difference between (6.39) and (6.42) only appears at $\mathcal{O}\left(\alpha_{s}\right)$. We note that only the symmetric part of $T_{a_{0} \rightarrow a_{1} a_{2}}$ survives the contraction with $\boldsymbol{y}^{l} \boldsymbol{y}^{l^{\prime}}$. As a consequence, combinations of $a_{1}, a_{2}$ with exactly one longitudinal polarisation give zero, since one then has $T^{l l^{\prime}} \propto \epsilon^{l l^{\prime}}$ (see section 7.4).

Little is known in phenomenology about twist-four distributions. The computation using models of light-cone wave functions in [108] can give guidance at large but not at small $x$. If one has a model for $F_{\text {int }}\left(x_{i}, \boldsymbol{y}\right)$ and is content with leading-order accuracy in $\alpha_{s}$, one may combine (6.39) and (6.42) to approximate

$$
{ }^{R} F_{\text {int }, a_{1} a_{2}}\left(x_{i}, \boldsymbol{z}_{i}, \boldsymbol{y} ; \mu, \mu, \zeta\right)={ }^{R} F_{\text {int }, a_{1} a_{2}}\left(x_{i}, \boldsymbol{y} ; \mu, \mu, \zeta\right)+\mathcal{O}\left(\alpha_{s}\right)
$$


and use the intrinsic part of the DPDF on the r.h.s. in the small-y contribution to the cross section, without further approximation. The scales $\mu$ and $\zeta$ should of course be chosen appropriately.

\subsubsection{Evolved DPDs and cross section}

Inserting the decomposition (6.37) of the DTMDs into (5.17) and neglecting twist-three distributions, we obtain

$$
\begin{aligned}
& { }^{R} F_{a_{1} a_{2}}\left(x_{i}, \boldsymbol{z}_{i}, \boldsymbol{y} ; \mu_{i}, \zeta\right) \\
& =\exp \left\{\int_{\mu_{0}}^{\mu_{1}} \frac{d \mu}{\mu}\left[\gamma_{a_{1}}(\mu)-\gamma_{K, a_{1}}(\mu) \log \frac{\sqrt{x_{1} \zeta / x_{2}}}{\mu}\right]+{ }^{1} K_{a_{1}}\left(\boldsymbol{z}_{1} ; \mu_{0}\right) \log \frac{\sqrt{\zeta}}{\sqrt{\zeta_{0}}}\right. \\
& \left.\quad+\int_{\mu_{0}}^{\mu_{2}} \frac{d \mu}{\mu}\left[\gamma_{a_{2}}(\mu)-\gamma_{K, a_{2}}(\mu) \log \frac{\sqrt{x_{2} \zeta / x_{1}}}{\mu}\right]+{ }^{1} K_{a_{2}}\left(\boldsymbol{z}_{2} ; \mu_{0}\right) \log \frac{\sqrt{\zeta}}{\sqrt{\zeta_{0}}}\right\} \\
& \quad \times \sum_{R^{\prime}} R R^{\prime} \exp \left[M_{a_{1} a_{2}}\left(\boldsymbol{z}_{i}, \boldsymbol{y}\right) \log \frac{\sqrt{\zeta}}{\sqrt{\zeta_{0}}}\right] \\
& \quad \times\left[{ }^{R^{\prime}} F_{a_{1} a_{2}, \operatorname{spl}}\left(x_{i}, \boldsymbol{z}_{i}, \boldsymbol{y} ; \mu_{0}, \mu_{0}, \zeta_{0}\right)+{ }^{R^{\prime}} F_{a_{1} a_{2}, \text { int }}\left(x_{i}, \boldsymbol{z}_{i}, \boldsymbol{y} ; \mu_{0}, \mu_{0}, \zeta_{0}\right)\right]
\end{aligned}
$$

as our master formula for DTMDs at small $\boldsymbol{z}_{i}$ and small $\boldsymbol{y}$. For the scales in the DTMDs we take

$$
\mu_{0}=\mu_{Z}, \quad \zeta_{0}=\mu_{Z}^{2}
$$

with $\mu_{Z}$ defined as in (6.26). We can then use the short-distance approximations (6.38) and (6.39) for $F_{\text {spl }}$ and $F_{\text {int }}$. The choice (6.45) will turn out to be well suited for combining the different contributions to the overall cross section. In contrast to (6.22), we have taken the two renormalisation scales $\mu_{01}$ and $\mu_{02}$ equal in (6.44), given that we do not have an optimised short-distance expansion of $F$ for different scales. One can however optimise the resummation of logarithms in the exponent by writing

$$
{ }^{1} K_{a_{1}}\left(\boldsymbol{z}_{1} ; \mu_{0}\right)={ }^{1} K_{a_{1}}\left(\boldsymbol{z}_{1} ; \mu_{01}\right)+\int_{\mu_{0}}^{\mu_{01}} \frac{d \mu}{\mu} \gamma_{K, a_{1}}(\mu)
$$

and likewise for ${ }^{1} K_{a_{2}}$. A natural choice for the additional scales is $\mu_{0 i}=\mu_{z_{i}}$, as already suggested for (6.22). Inserting the evolved DTMDs into (5.19), we have

$$
\begin{aligned}
W_{\text {small } y}= & \exp \left\{\int_{\mu_{0}}^{\mu_{1}} \frac{d \mu}{\mu}\left[\gamma_{a_{1}}(\mu)-\gamma_{K, a_{1}}(\mu) \log \frac{Q_{1}^{2}}{\mu^{2}}\right]+{ }^{1} K_{a_{1}}\left(\boldsymbol{z}_{1}, \mu_{0}\right) \log \frac{Q_{1} Q_{2}}{\zeta_{0}}\right. \\
& \left.+\int_{\mu_{0}}^{\mu_{2}} \frac{d \mu}{\mu}\left[\gamma_{a_{2}}(\mu)-\gamma_{K, a_{2}}(\mu) \log \frac{Q_{2}^{2}}{\mu^{2}}\right]+{ }^{1} K_{a_{2}}\left(\boldsymbol{z}_{2}, \mu_{0}\right) \log \frac{Q_{1} Q_{2}}{\zeta_{0}}\right\} \\
\times & \sum_{R R^{\prime}}\left[{ }^{R} F_{b_{1} b_{2}, \operatorname{spl}}\left(\bar{x}_{i}, \boldsymbol{z}_{i}, \boldsymbol{y} ; \mu_{0}, \mu_{0}, \zeta_{0}\right)+{ }^{R} F_{b_{1} b_{2}, \text { int }}\left(\bar{x}_{i}, \boldsymbol{z}_{i}, \boldsymbol{y} ; \mu_{0}, \mu_{0}, \zeta_{0}\right)\right] \\
& \times \Phi\left(\nu \boldsymbol{y}_{+}\right) \Phi\left(\nu \boldsymbol{y}_{-}\right) \eta_{a_{1} a_{2}}(R) R R^{\prime} \exp \left[M_{a_{1} a_{2}}\left(\boldsymbol{z}_{i}, \boldsymbol{y}\right) \log \frac{Q_{1} Q_{2}}{\zeta_{0}}\right] \\
& \times\left[{ }^{R^{\prime}} F_{a_{1} a_{2}, \operatorname{spl}}\left(x_{i}, \boldsymbol{z}_{i}, \boldsymbol{y} ; \mu_{0}, \mu_{0}, \zeta_{0}\right)+{ }^{R} F_{a_{1} a_{2}, \operatorname{int}}\left(x_{i}, \boldsymbol{z}_{i}, \boldsymbol{y} ; \mu_{0}, \mu_{0}, \zeta_{0}\right)\right]
\end{aligned}
$$

at the cross section level. 
At present, one can only use the leading order (LO) expressions (6.38) and (6.39) of the DTMD expansions, because the next-to-leading order (NLO) terms have not been computed for either case. The leading $\mathcal{O}\left(\alpha_{s}\right)$ term of $F_{\text {spl }}$ depends on $\boldsymbol{y}_{ \pm}$but not on $\boldsymbol{Z}$, and the leading $\mathcal{O}\left(\alpha_{s}^{0}\right)$ expression for $F_{\text {int }}$ is independent of any transverse distance. A $\boldsymbol{Z}$ dependence via $\mu_{0}$ and $\zeta_{0}$ cancels in $W_{\text {small } y}$, up to orders in $\alpha_{s}$ beyond the accuracy of the calculation. However, $W_{\text {small } y}$ acquires a dependence on $\boldsymbol{Z}$ through the $\boldsymbol{z}_{1}$ and $\boldsymbol{z}_{2}$ dependence of the Collins-Soper kernels ${ }^{1} K_{a_{1}}\left(\boldsymbol{z}_{1} ; \mu_{0}\right),{ }^{1} K_{a_{2}}\left(\boldsymbol{z}_{2} ; \mu_{0}\right)$ and $M_{a_{1} a_{2}}\left(\boldsymbol{z}_{i}, \boldsymbol{y}\right)$. Since these kernels start at $\mathcal{O}\left(\alpha_{s}\right)$, the corresponding $\boldsymbol{Z}$ dependence comes with a large logarithm $\log \left(Q_{1} Q_{2} / \zeta_{0}\right)$ for each power of $\alpha_{s}$ and is thus enhanced compared to the $\alpha_{s}$ corrections in $F_{\text {spl }}$ and $F_{\text {int }}$, so that it is consistent to keep the former while neglecting the latter. In this way, rapidity evolution provides a nontrivial $\boldsymbol{Z}$ dependence and thus a nontrivial dependence on the conjugate momentum $\boldsymbol{q}_{1}+\boldsymbol{q}_{2}$ in the cross section, even if one uses the short-distance expansions (6.38) and (6.39) at leading order. In physical terms, this is because the exponential in (6.47) resums graphs for the soft factor that have a real gluon emission enhanced by large rapidity logarithms, even though the graphs giving the leading-order expansions of $F_{\text {spl }}$ and $F_{\text {int }}$ have no real gluon emission into the final state.

\subsection{Combining large and small $y$}

As we have seen in the previous subsections, the different regions of $\boldsymbol{z}_{1}, \boldsymbol{z}_{2}$ and $\boldsymbol{y}$ contributing to the cross section involve different approximations, whose results are given in (6.24) and (6.47). An important point is that the approximated expressions give leading-power contributions not only in the regions for which they are designed, but also outside these regions. With the short-distance behaviour $F\left(x_{i}, \boldsymbol{y}\right) \sim 1 / \boldsymbol{y}^{2}$ following from (6.42) we see for instance that the integral of $W_{\text {large } y}$ over $\boldsymbol{y}$ extends down to the smallest values allowed by the cutoff function $\Phi(\nu \boldsymbol{y})$, where the approximations used to obtain the expression are clearly invalid.

To deal with this problem we adapt the subtraction formalism discussed in chapter 10 of [65], which we briefly sketch now. The formalism is formulated for a given Feynman graph $\Gamma$ in momentum space. It starts with the smallest regions of loop momenta and works its way towards increasingly larger regions. In this context, a region $r^{\prime}$ is called smaller than $r$ (i.e. $r^{\prime}<r$ ) if hard momenta in $r$ are collinear or soft in $r^{\prime}$, or if collinear momenta in $r^{\prime}$ are soft in $r$. A more general definition of the relation $r^{\prime}<r$ is discussed in chapters 5.4.1 and 10.1.3 of [65]. For each region $r$ that gives a leading contribution to the cross section, a set of approximations $T_{r}$ is applied to the graph. In each approximated term $T_{r} \Gamma$ one still integrates over all loop momenta, thus avoiding momentum cutoffs. This leads to double counting of contributions from the overlap between different regions, which are recursively removed by subtraction terms. The full graph is then approximated by

$$
\Gamma \approx \sum_{r} C_{r} \Gamma \quad \text { with } \quad C_{r} \Gamma=T_{r} \Gamma-\sum_{r^{\prime}<r} T_{r} C_{r^{\prime}} \Gamma .
$$

$C_{r} \Gamma$ provides a valid approximation of $\Gamma$ in the region $r$ and in all smaller regions $r^{\prime}<r$, thanks to the subtraction terms $T_{r} C_{r^{\prime}} \Gamma$, which are obtained by applying the approximations for both $r$ and the smaller regions. In the case where one has only two regions $r^{\prime}<r$, 


\begin{tabular}{|ccc|}
\hline region & power counting & approximations \\
\hline DPS, large $\boldsymbol{y}$ & $|\boldsymbol{y}|,\left|\boldsymbol{y}_{+}\right|,\left|\boldsymbol{y}_{-}\right| \sim 1 / \Lambda$ & $\left|\boldsymbol{z}_{i}\right| \ll|\boldsymbol{y}|, 1 / \Lambda$ \\
DPS, small $\boldsymbol{y}$ & $|\boldsymbol{y}|,\left|\boldsymbol{y}_{+}\right|,\left|\boldsymbol{y}_{-}\right| \sim 1 / q_{T}$ & $\left|\boldsymbol{z}_{i}\right|,|\boldsymbol{y}| \ll 1 / \Lambda$ \\
SPS & $\left|\boldsymbol{y}_{+}\right|,\left|\boldsymbol{y}_{-}\right| \sim 1 / Q$ & $\left|\boldsymbol{y}_{+}\right|,\left|\boldsymbol{y}_{-}\right| \ll\left|\boldsymbol{z}_{i}\right| \ll 1 / \Lambda$ \\
\hline
\end{tabular}

Table 1. Regions of $\boldsymbol{y}$ discussed in the text. For power counting, $\left|\boldsymbol{z}_{i}\right| \sim 1 / q_{T}$ is always assumed.

the subtraction term is simply $T_{r} T_{r^{\prime}} \Gamma$. In [65] it was shown that if the approximation $T_{r}$ is good up to power corrections in $\Lambda / Q$ in its design region $r$, then $\sum_{r} C_{r} \Gamma$ is good with the same accuracy for the full graph.

In [30], this formalism was applied to the double counting problem between single and double parton scattering, leading to the formulation we already presented in section 5.3. At variance to the original formulation in [65], momentum regions in (6.48) were replaced by regions of the transverse distances $\boldsymbol{y}_{+}$and $\boldsymbol{y}_{-}$, starting from the region of large distances and going towards small ones in the recursive construction of subtraction terms.

We now use the same procedure to remove the double counting between the regions of "large" and "small" $\boldsymbol{y}$ as characterised in table 1. The treatment of the regions where $\left|\boldsymbol{y}_{+}\right|$or $\left|\boldsymbol{y}_{-}\right|$are of order $1 / Q$ is postponed to the next subsection. It is always understood that $\left|z_{1}\right|$ and $\left|z_{2}\right|$ are in the perturbative region, of order $1 / q_{T}$, as discussed in section 6.1.

According to the general construction, the subtraction term for large $\boldsymbol{y}$ in the small- $\boldsymbol{y}$ term is obtained by applying the approximations in the first and second row of table 1 , i.e. by using both $\left|\boldsymbol{z}_{i}\right| \ll|\boldsymbol{y}|$ and $|\boldsymbol{y}| \ll 1 / \Lambda$. For a given Feynman graph (and thus for any finite sum over graphs) the order in which these approximations are made does not matter. This suggests that we can start from either $W_{\text {large } y}$ or $W_{\text {small } y}$ and then apply the approximation for the other region. At this point, we must however note that the expressions of $W$ are not for fixed-order graphs but contain logarithms resummed to all orders in the strong coupling. This is reflected in the scale dependence of $\alpha_{s}$ and of the nonperturbative functions. We must therefore make sure that the choice of scales in the different terms is compatible with the way in which the subtraction formalism works. In $W_{\text {small } y}$, this concerns the scales $\mu_{0}$ and $\zeta_{0}$ at which one evaluates the short-distance expansions (6.38) and (6.39) of DTMDs at fixed order in $\alpha_{s}$.

We now define the double counting subtraction term as

$$
W_{\text {sub }}=\left.W_{\text {small } y}\right|_{\text {approx. for }\left|\boldsymbol{z}_{i}\right| \ll|\boldsymbol{y}|}
$$

with the small-y expression for $W$ given in (6.47). We should take the limit $\left|\boldsymbol{z}_{1}\right|,\left|\boldsymbol{z}_{2}\right| \ll|\boldsymbol{y}|$ in all parts of that expression. In particular, this means replacing $\boldsymbol{y}_{ \pm}$with $\boldsymbol{y}$ in $\Phi$ and in the leading-order term of the short-distance expansion (6.38) of $F_{\text {spl }}$, and replacing ${ }^{R R^{\prime}} M_{a_{1} a_{2}}$ with $\delta_{R R^{\prime}}\left[{ }^{R} K_{a_{1}}+{ }^{R} K_{a_{2}}-{ }^{1} K_{a_{1}}-{ }^{1} K_{a_{2}}+{ }^{R} J\right]$ according to (5.16) and (6.16).

We note at this point that the integral of (6.47) over $\boldsymbol{y}$ is actually divergent at large $\boldsymbol{y}$. Up to logarithmic corrections, the $2 \mathrm{v} 2$ term $F_{\text {int }} \times F_{\text {int }}$ is constant and the $2 \mathrm{v} 1$ term $F_{\text {spl }} \times F_{\text {int }}$ goes like $1 / \boldsymbol{y}^{2}$ in that limit, and for $R=R^{\prime}=1$ there is no large- $\boldsymbol{y}$ suppression from the exponential since ${ }^{1} J=0$. The construction (6.49) ensures that the region $|\boldsymbol{y}| \gg 1 / q_{T}$ 
cancels in $W_{\text {small } \boldsymbol{y}}-W_{\text {sub }}$. When evaluating the cross section numerically, one may want to use an upper cutoff of order $1 / \Lambda$ on the integral over $|\boldsymbol{y}|$, thus avoiding to evaluate terms in a region where they cancel anyway.

For the subtraction formalism to work, we need that

$$
W_{\text {sub }} \approx W_{\text {large } \boldsymbol{y}} \text { for }|\boldsymbol{y}| \sim\left|\boldsymbol{z}_{1}\right|,\left|\boldsymbol{z}_{2}\right|
$$

up to terms in $\alpha_{s}$ that are beyond the accuracy of the calculation. In the specified region of $\boldsymbol{y}$, we can use the short-distance limit of $J(\boldsymbol{y})$ and of the DPDFs in (6.24), the latter being obtained by adding $F_{\mathrm{spl}}$ and $F_{\text {int }}$ from (6.42). If one retains only the leading-order terms for $F_{\mathrm{spl}}+F_{\text {int }}$ on both sides of (6.50), one finds manifest equality of the two expressions up to differences due to scale dependence, which we will discuss shortly.

The full DPS cross section is then obtained from

$$
W_{\text {DPS }}(\nu)=W_{\text {large } y}\left(\nu^{\prime}\right)-W_{\text {sub }}\left(\nu^{\prime}\right)+W_{\text {small } y}(\nu),
$$

where we make explicit the choice of cutoff parameters for the $\boldsymbol{y}$ integration, taking $\nu^{\prime} \sim q_{T}$ and $\nu \sim Q$. In the region $|\boldsymbol{y}| \gg\left|\boldsymbol{z}_{1}\right|,\left|\boldsymbol{z}_{2}\right|$ the last two terms cancel by virtue of (6.49), and one is left with the first term, which is designed to give a correct approximation of the cross section there. For $|\boldsymbol{y}| \sim\left|\boldsymbol{z}_{1}\right|,\left|\boldsymbol{z}_{2}\right|$, the first and second terms cancel according to (6.50), and the third term gives a correct approximation of the cross section. In this way, $W_{\text {DPS }}$ leads to a correct approximation of the cross section for $|\boldsymbol{y}|$ of order $1 / q_{T}$ and larger.

We can take a more restrictive cutoff parameter $\nu^{\prime} \sim q_{T}$ in the first two terms, because according to (6.50) the sum of these terms is already suppressed for $|\boldsymbol{y}| \sim 1 / q_{T}$, and the dependence on the exact value of $\nu^{\prime}$ is thus beyond the accuracy of the calculation. Taking $\nu^{\prime}$ lower than $Q$ is also more economical for numerical calculations since it excludes a $\boldsymbol{y}$ region that is not needed. By contrast, $\nu$ in the last term should be of order $Q$ (see the next subsection).

Returning to the issue of scale dependence, we should first specify what is to be used for the nonperturbative quantities in the expression (6.24) of $W_{\text {large } y}$. For the Collins-Soper kernel we take the form in $(6.28)$ with $J\left(\boldsymbol{y}^{*} ; \mu_{y}, \mu_{y}\right)$ and $\gamma_{J}$ evaluated at fixed perturbative order; this ensures the correct small- $\boldsymbol{y}$ limit. Using the DGLAP equations, the DPDFs are to be evolved from $\mu_{0 i}$ to $\mu_{0}=\sqrt{\zeta_{0}}=\mu_{y}$, and it is at that scale that one makes an ansatz which at small $\boldsymbol{y}$ tends to the fixed-order form specified by (6.42). ${ }^{12}$ As usual, this scale choice ensures that higher-order corrections are not accompanied by large logarithms.

We now recall that in $W_{\text {small } y}$, and hence by construction also in $W_{\text {sub }}$, we take $\mu_{0}=$ $\sqrt{\zeta_{0}}=\mu_{Z}$ for the scales at which we perform the short-distance expansion of the DTMDs. The difference between the two sides in (6.50) is thus due to different scales of the shortdistance matching and to the different scales at which the DPDs and the Collins-Soper kernels are evaluated in (6.24) and (6.47). However, all these scales, $\mu_{y}, \mu_{z_{i}}$ and $\mu_{Z}$ are of the same order in the region $|\boldsymbol{y}| \sim\left|\boldsymbol{z}_{i}\right|$ where we need (6.50). Using the same methods as in section 6.2 of [30], one can show that the approximation (6.50) holds up to higherorder terms in $\alpha_{s}$. These terms are not accompanied by large logarithms and beyond the accuracy of the calculation.

\footnotetext{
${ }^{12}$ An explicit example for such an ansatz in the colour singlet sector can be found in section 9.2 of [30].
} 


\begin{tabular}{|cc|}
\hline partial cross section & approximations \\
\hline$\sigma_{\text {DPS } / \text { SPS }}$ & $\left|\boldsymbol{y}_{+}\right| \ll 1 / \Lambda$ \\
$\sigma_{\text {DPS } / \text { SPS }, y_{+} \rightarrow 0}$ & $\left|\boldsymbol{y}_{+}\right| \ll 1 / q_{T}$ \\
$\sigma_{\text {DPS }, y_{-} \rightarrow 0}$ & $\left|\boldsymbol{y}_{-}\right| \ll 1 / q_{T},\left|\boldsymbol{y}_{+}\right| \ll 1 / \Lambda$ \\
$\sigma_{\text {DPS }, y_{ \pm} \rightarrow 0}$ & $\left|\boldsymbol{y}_{-}\right|,\left|\boldsymbol{y}_{+}\right| \ll 1 / q_{T}$ \\
\hline
\end{tabular}

Table 2. Conditions on $\boldsymbol{y}_{ \pm}$for different terms in the overall cross section (5.30) if $q_{T} \gg \Lambda$. Analogous relations hold for $\sigma_{\mathrm{SPS} / \mathrm{DPS}}$ and the associated subtraction terms. In all cases one has $|\boldsymbol{Z}| \ll 1 / \Lambda$.

\subsection{Combining DPS with SPS at short distances}

In section 5.3 we recalled how to combine DPS, SPS and their interference in TMD factorisation at generic values of $q_{T}$. We now derive the form of the double counting subtraction terms for the case where $q_{T} \gg \Lambda$. We restrict ourselves to the lowest order of $\alpha_{s}$ in the perturbative short-distance coefficients. An extension to higher orders is beyond the scope of the present work.

Let us start by discussing the relevant transverse distance scales and the approximations they imply. We recall that the DPS cross section for $q_{T} \gg \Lambda$ is dominated by distances $\left|\boldsymbol{z}_{1}\right|,\left|\boldsymbol{z}_{2}\right| \ll 1 / \Lambda$ thanks to the Fourier exponentials in (5.1). In full analogy, the Fourier exponentials in (5.31) and (5.32) ensure that $\sigma_{\text {DPS/SPS }}$ is dominated by $|\boldsymbol{Z}|,\left|\boldsymbol{y}_{+}\right| \ll 1 / \Lambda$ and $\sigma_{\text {SPS/DPS }}$ by $|\boldsymbol{Z}|,\left|\boldsymbol{y}_{-}\right| \ll 1 / \Lambda$. Likewise, $\sigma_{\text {SPS }}$ is dominated by $|\boldsymbol{Z}| \ll 1 / \Lambda$, where in the following we use the variable $\boldsymbol{Z}$ instead of $\boldsymbol{z}$ in the SPS formula (2.1). According to the formalism recalled in the previous subsection, the subtraction terms for the overlap between double and single parton scattering are obtained by applying the approximations appropriate for the SPS region to the DPS term in the cross section. A corresponding prescription holds for the SPS/DPS interference. The limits $\boldsymbol{y}_{+} \rightarrow \mathbf{0}, \boldsymbol{y}_{-} \rightarrow \mathbf{0}$ or $\boldsymbol{y}_{ \pm} \rightarrow \mathbf{0}$ in the subtraction terms hence imply that these distances are taken to be much smaller than $1 / q_{T}$, because $q_{T}$ is set to zero when one computes the hard scattering process for SPS. We thus obtain the approximations given in table 2 . The constraint on $\boldsymbol{y}_{+}$in the third row results from $\boldsymbol{y}_{+}=\boldsymbol{y}_{-}+$ $\left(\boldsymbol{z}_{1}-\boldsymbol{z}_{2}\right)$ and the constraints on $\boldsymbol{y}_{-}, \boldsymbol{z}_{1}$ and $\boldsymbol{z}_{2}$. We thus find that all terms in the overall cross section (5.30) are dominated by distances in the perturbative region, except for $\sigma_{\mathrm{DPS}}$.

In all terms that are dominated by short distances, we can use the perturbative splitting expressions (5.35) and (5.36) of the twist-three TMDs and the DTMDs. Notice that the LO terms in these expressions have a factorised dependence on $\boldsymbol{y}_{+}, \boldsymbol{y}_{-}$and $\boldsymbol{Z}$, so that they do not depend on the relative size of these distances (this will no longer hold at NLO). Moreover, we can take the short-distance limit of the TMDs $f_{a}(x, Z ; \mu, \zeta)$. At LO accuracy we can simply replace them with collinear PDFs $f_{a}(x ; \mu)$, provided that $\mu^{2} \sim \zeta \sim 1 / Z^{2}$. Collinear PDFs are then the only nonperturbative input necessary to compute the cross section, apart of course from the DPDs and Collins-Soper kernels $J$ in $\sigma_{\text {DPS }}$. In a complete treatment, one would also have collinear twist-three distributions, but we neglect these as explained in section 5.3. 
From the dependence on $\boldsymbol{y}_{ \pm}$of the splitting expressions (5.35) and (5.36) and from the cross section formulae (2.1), (5.31) and (5.32) we deduce that the SPS cross section, the SPS/DPS interference and all associated subtraction terms have the same power behaviour as the $1 \mathrm{v} 1$ term of the DPS cross section in (6.40), namely $Q^{4} d \sigma /\left(d^{2} \boldsymbol{q}_{1} d^{2} \boldsymbol{q}_{2}\right) \sim 1 / q_{T}^{2}$.

We must now take a closer look at the scale dependence of the distributions. Let us denote by $\mu_{h}$ the hard scale chosen in the computation of the SPS amplitude, and by $\mu_{1}$ and $\mu_{2}$ the scales for the two DPS amplitudes. To simplify the presentation, we first assume that all hard scales are equal, $\mu=\mu_{h}=\mu_{1}=\mu_{2}$. The adaptation to the case of different hard scales is discussed in appendix C. As we have explicitly shown for single and double parton TMDs, the evolution in $\mu$ and $\zeta$ of these distributions is multiplicative:

$$
\begin{aligned}
f_{a}(x, \boldsymbol{z} ; \mu, \zeta) & =E_{2 ; a}\left(\boldsymbol{z} ; \mu, \zeta ; \mu_{0}, \zeta_{0}\right) f_{a}\left(x, \boldsymbol{z} ; \mu_{0}, \zeta_{0}\right), \\
F_{a_{1} a_{2}}\left(x_{i}, \boldsymbol{z}_{i}, \boldsymbol{y} ; \mu, \zeta\right) & =E_{4 ; a_{1} a_{2}}\left(x_{i}, \boldsymbol{z}_{i}, \boldsymbol{y} ; \mu, \zeta ; \mu_{0}, \zeta_{0}\right) F_{a_{1} a_{2}}\left(x_{i}, \boldsymbol{z}_{i}, \boldsymbol{y} ; \mu_{0}, \zeta_{0}\right),
\end{aligned}
$$

where it is understood that $F$ is a vector and the evolution factor $E_{4}$ a matrix in the space of colour representations $R$. From the discussion at the end of section 5.3 if follows that for the twist-three TMDs, we have

$$
D_{\alpha_{1} \alpha_{2} \mid \alpha_{0}}\left(x_{i}, \boldsymbol{y}_{+}, \boldsymbol{Z} ; \mu, \zeta\right)=E_{3 ; \alpha_{1} \alpha_{2} \mid \alpha_{0}}\left(x_{i}, \boldsymbol{y}_{+}, \boldsymbol{Z} ; \mu, \zeta ; \mu_{0}, \zeta_{0}\right) D_{\alpha_{1} \alpha_{2} \mid \alpha_{0}}\left(x_{i}, \boldsymbol{y}_{+}, \boldsymbol{Z} ; \mu_{0}, \zeta_{0}\right)
$$

and an analogous equation for $D_{\alpha_{0} \mid \alpha_{1} \alpha_{2}}$.

Let us now specify the evolution and scale dependence of the distributions appearing in $\sigma_{\mathrm{SPS}}$, the interference terms and $\sigma_{\mathrm{DPS}}$ in the region where all transverse distances are small compared with $1 / \Lambda$. In a schematic notation, we have

$$
\begin{aligned}
& \sigma_{\mathrm{SPS}}: \quad f\left(x_{1}+x_{2}, \boldsymbol{Z} ; \mu\right) \sim E_{2}\left(\mu ; \mu_{Z}\right) f\left(\mu_{Z}\right), \\
& \sigma_{\mathrm{DPS} / \mathrm{SPS}}: \quad D\left(x_{i}, \boldsymbol{y}_{+}, \boldsymbol{Z} ; \mu\right) \sim E_{3}\left(\mu ; \mu_{Z}\right) U\left(\mu_{Z}\right) f\left(\mu_{Z}\right), \\
& \sigma_{\mathrm{SPS} / \mathrm{DPS}}: \quad D\left(x_{i}, \boldsymbol{y}_{-}, \boldsymbol{Z} ; \mu\right) \sim E_{3}\left(\mu ; \mu_{Z}\right) U^{*}\left(\mu_{Z}\right) f\left(\mu_{Z}\right) \text {, } \\
& \sigma_{\mathrm{DPS}}: \quad F\left(x_{i}, \boldsymbol{z}_{i}, \boldsymbol{y} ; \mu\right) \sim E_{4}\left(\mu ; \mu_{Z}\right) U\left(\mu_{Z}\right) U^{*}\left(\mu_{Z}\right) f\left(\mu_{Z}\right)+F_{\text {int }}(\mu),
\end{aligned}
$$

where $f\left(\mu_{Z}\right)$ on the r.h.s. is a collinear PDF. We have specified only the splitting part $F_{\mathrm{spl}}$ of $F$, because the detailed form of $F_{\text {int }}$ will not be needed. For brevity we have omitted parton labels, as well as momentum fraction and transverse position arguments on the r.h.s. The factors $\boldsymbol{y}_{+}^{l} / \boldsymbol{y}_{+}^{2}$ and $\boldsymbol{y}_{-}^{l^{\prime}} / \boldsymbol{y}_{-}^{2}$ which accompany the splitting kernels $U\left(\mu_{Z}\right)$ and $U^{*}\left(\mu_{Z}\right)$ have been omitted as well. For brevity, we display neither the rapidity parameter $\zeta$ in the evolved distributions nor the starting value $\zeta_{0}$. For the latter, we follow (6.45) and take $\zeta_{0}=\mu_{Z}^{2}$. Since $\zeta$ is defined by (5.8), it must be rescaled by $\left(x_{1}+x_{2}\right)^{2} \zeta /\left(x_{1} x_{2}\right)$ when used in $f\left(x_{1}+x_{2}, \boldsymbol{Z}\right)$ and in $E_{2}$. The same forms as in (6.54) are to be taken for the distributions in the left-moving proton, with $x_{i}$ replaced by $\bar{x}_{i}$ and $\zeta$ by $\bar{\zeta}$. 
The subtraction terms should be such that, up to higher orders in $\alpha_{s}$ and up to power corrections, the overall cross section (5.30) is given by

$$
\begin{array}{r}
d \sigma_{\mathrm{DPS}} \text { for }\left|\boldsymbol{y}_{+}\right| \sim\left|\boldsymbol{y}_{-}\right| \gg 1 / \nu, \\
d \sigma_{\mathrm{SPS}} \text { for }\left|\boldsymbol{y}_{+}\right| \sim\left|\boldsymbol{y}_{-}\right| \sim 1 / \nu, \\
d \sigma_{\mathrm{DPS} / \mathrm{SPS}} \text { for }\left|\boldsymbol{y}_{+}\right| \gg\left|\boldsymbol{y}_{-}\right| \sim 1 / \nu, \\
d \sigma_{\mathrm{SPS} / \mathrm{DPS}} \text { for }\left|\boldsymbol{y}_{-}\right| \gg\left|\boldsymbol{y}_{+}\right| \sim 1 / \nu,
\end{array}
$$

where it is understood that $\nu \sim Q$. In the subtraction terms, renormalisation and rapidity scales should be chosen such that the cancellations required to avoid double counting are not spoiled by large logarithms at higher orders.

To achieve this, we take so-called profile scales, which vary smoothly between $\mu_{Z}$ and $\mu$ as a function of the appropriate transverse distance. We therefore introduce a profile function $p$ that satisfies

$$
\begin{aligned}
& p\left(u ; \mu_{a}, \mu_{b}\right) \approx \mu_{a} \quad \text { for } u \sim 1, \\
& p\left(u ; \mu_{a}, \mu_{b}\right) \approx \mu_{b} \quad \text { for } u \gg 1 \text {; }
\end{aligned}
$$

a concrete example is given below. For the subtraction terms between DPS and the SPS/DPS interference, we now take

$$
\begin{aligned}
& \sigma_{\mathrm{DPS} / \mathrm{SPS}, y_{+} \rightarrow 0}: D \sim E_{3}(\mu ; \hat{\mu}) U(\hat{\mu}) E_{2}\left(\hat{\mu} ; \mu_{Z}\right) f\left(\mu_{Z}\right) \quad \text { with } \hat{\mu}=p\left(\nu\left|\boldsymbol{y}_{+}\right| ; \mu_{Z}, \mu\right), \\
& \sigma_{\mathrm{SPS} / \mathrm{DPS}, y_{-} \rightarrow 0}: D \sim E_{3}(\mu ; \hat{\mu}) U^{*}(\hat{\mu}) E_{2}\left(\hat{\mu} ; \mu_{Z}\right) f\left(\mu_{Z}\right) \quad \text { with } \hat{\mu}=p\left(\nu\left|\boldsymbol{y}_{-}\right| ; \mu_{Z}, \mu\right) .
\end{aligned}
$$

This corresponds to a two-step matching: at the scale $\mu_{Z}$ a twist-two TMD is matched onto a PDF. The twist-two TMD is then evolved up to the scale $\hat{\mu}$, where a twist-three TMD is matched onto the twist-two TMD using the lowest order term in (5.35). The twist-three TMD is then evolved up to the final scale $\mu$. It is understood that at the intermediate scale $\hat{\mu}$ the rapidity parameter is taken equal to

$$
\widehat{\zeta}=p\left(u ; \mu_{Z}^{2}, \zeta\right)
$$

where the first argument $u$ is the same as the first argument in $\hat{\mu}$. We thus find that

$$
\sigma_{\mathrm{DPS} / \mathrm{SPS}, y_{+} \rightarrow 0} \approx \begin{cases}\sigma_{\mathrm{DPS} / \mathrm{SPS}} & \text { for }\left|\boldsymbol{y}_{+}\right| \sim 1 / \nu \\ \sigma_{\mathrm{SPS}} & \text { for }\left|\boldsymbol{y}_{+}\right| \gg 1 / \nu\end{cases}
$$

where we have used that for distances $\left|\boldsymbol{y}_{+}\right| \gg 1 / Q$ the hard scattering amplitude for SPS can be written as

$$
\left.H_{\alpha_{0} \beta_{0}}\right|_{y_{+} \gg 1 / Q}=\frac{\boldsymbol{y}_{+}^{k}}{\boldsymbol{y}_{+}^{2}} \frac{\boldsymbol{y}_{+}^{l}}{\boldsymbol{y}_{+}^{2}} U_{\alpha_{0} \rightarrow \alpha_{1} \alpha_{2}}^{k} U_{\beta_{0} \rightarrow \beta_{1} \beta_{2}}^{l} H_{\alpha_{1} \beta_{1}} H_{\alpha_{2} \beta_{2}}
$$

at leading order in $\alpha_{s}$. To make this relation explicit, one needs to Fourier transform the appropriate transverse momentum in the SPS graph to position space. For diagrams that have kinematic overlap between SPS and the SPS/DPS interference, but not with DPS, 
such as the one in figure $5 \mathrm{~b}$, the behaviour in (6.59) and in its analogue for $\sigma_{\mathrm{SPS} / \mathrm{DPS}, y_{-} \rightarrow 0}$ solve the double counting problem completely. If there is also overlap with the DPS region, as in the three graphs of figure 4, then we need the DPS subtraction terms, where we choose scales as follows:

$$
\begin{aligned}
& \sigma_{\mathrm{DPS}, y_{-} \rightarrow 0}: F \sim E_{4}(\mu ; \hat{\mu}) U^{*}(\hat{\mu}) E_{3}\left(\hat{\mu} ; \mu_{Z}\right) U\left(\mu_{Z}\right) f\left(\mu_{Z}\right), \\
& \sigma_{\mathrm{DPS}, y_{+} \rightarrow 0}: F \sim E_{4}(\mu ; \hat{\mu}) U(\hat{\mu}) E_{3}\left(\hat{\mu} ; \mu_{Z}\right) U^{*}\left(\mu_{Z}\right) f\left(\mu_{Z}\right), \\
& \sigma_{\mathrm{DPS}, y_{ \pm} \rightarrow 0}: F \sim E_{4}(\mu ; \hat{\mu}) U(\hat{\mu}) U^{*}(\hat{\mu}) E_{2}\left(\hat{\mu} ; \mu_{Z}\right) f\left(\mu_{Z}\right)
\end{aligned}
$$

with

$$
\hat{\mu}=p\left(\nu \min \left\{\left|\boldsymbol{y}_{+}\right|,\left|\boldsymbol{y}_{-}\right|\right\} ; \mu_{Z}, \mu\right) .
$$

In all three terms we have a two-step matching, with a twist-three TMD at the intermediate stage in the first two cases and with a twist-two TMD in the third case. If both $\left|\boldsymbol{y}_{+}\right|$and $\left|\boldsymbol{y}_{-}\right|$are large compared with $1 / \nu$, we thus have

$$
\sigma_{\mathrm{DPS}, y_{-} \rightarrow 0} \approx \sigma_{\mathrm{DPS} / \mathrm{SPS}}, \quad \sigma_{\mathrm{DPS}, y_{+} \rightarrow 0} \approx \sigma_{\mathrm{SPS} / \mathrm{DPS}}, \quad \sigma_{\mathrm{DPS}, y_{ \pm} \rightarrow 0} \approx \sigma_{\mathrm{SPS}}
$$

so that the sum in (5.30) leaves only $\sigma_{\mathrm{DPS}}$, as it should. It remains to discuss the regions where one or both of $\left|\boldsymbol{y}_{+}\right|$and $\left|\boldsymbol{y}_{-}\right|$is of order $1 / \nu$. In each of these regions, $\sigma_{\text {DPS }}$ can be obtained from $W_{\text {small } y}$, because $|\boldsymbol{y}| \ll 1 / \Lambda$. Furthermore, the integral over $W_{\text {small } y}$ in these regions is dominated by the $1 \mathrm{v} 1$ term, with the $2 \mathrm{v} 1$ and $2 \mathrm{v} 2$ terms being power suppressed by at least a relative factor $\Lambda^{2} /\left(\nu q_{T}\right)$. This follows from the dependence of $F_{\mathrm{spl}}$ and $F_{\text {int }}$ on $\boldsymbol{y}_{+}$and $\boldsymbol{y}_{-}$. Finally, one finds that all three terms in (6.61) are approximately equal to the $1 \mathrm{v} 1$ term in $\sigma_{\mathrm{DPS}}$, which is thus cancelled in the combination (5.30). This leaves us with $\sigma_{\mathrm{SPS}}, \sigma_{\mathrm{DPS} / \mathrm{SPS}}$ or $\sigma_{\mathrm{SPS} / \mathrm{DPS}}$ as appropriate. Overall we thus have achieved a correct description of the cross section in all relevant regions of $\boldsymbol{y}_{+}$and $\boldsymbol{y}_{-}$.

Profile scales have been used in other contexts, see e.g. [109, 110]. For our purpose here, a suitable profile $p$ for $\hat{\mu}$ is given in equation (6.33) of [30]. An alternative choice is

$$
p\left(u ; \mu_{a}, \mu_{b}\right)= \begin{cases}\mu_{a} & \text { for } u \leq u_{a} \\ {\left[1-\frac{1}{2}\left(1-\cos u^{\prime} \pi\right)\right] \mu_{a}+\frac{1}{2}\left(1-\cos u^{\prime} \pi\right) \mu_{b}} & \text { for } u_{a}<u<u_{b} \\ \mu_{b} & \text { for } u \geq u_{b}\end{cases}
$$

with

$$
u^{\prime}=\frac{u-u_{a}}{u_{b}-u_{a}} .
$$

The transition points for the $u$ dependence should be such that $u_{a} \sim 1$ and $u_{b} \gg 1$. A natural choice is $u_{a}=\nu / \mu$ and $u_{b}=\nu / \mu_{Z}$.

\subsection{Perturbative accuracy}

Equations (2.11), (6.22) and (6.44) express TMDs in terms of collinear distributions and perturbative quantities, and they permit the resummation of large logarithms in the cross 
section. In the following, we give a brief overview of the order in $\alpha_{s}$ at which the different quantities are available. We also take a closer look at the type of logarithms that are resummed at all orders. To this end, we count all large scales $\mu_{i}, \zeta, \nu$ as order $Q$. Logarithms of $\left|\boldsymbol{z}_{i}\right|$ turn into logarithms of $\left|\boldsymbol{q}_{i}\right|$ after Fourier transformation, and we count $\mu_{i 0} \sim 1 /\left|\boldsymbol{z}_{i}\right| \sim q_{T}$.

An important point in this context is that a variety of schemes for TMD factorisation and for the associated resummation are used in the literature. Some care is needed when converting perturbative expressions from one scheme to another. A systematic discussion of this issue is given in [68], along with a comprehensive list of higher-order results for the quark channel in the scheme we use here (referred to as CSS2 in that paper).

SPS. The SPS cross section can be calculated within the single TMD formalism outlined in section 2. To compute the spectrum in $\boldsymbol{q}_{1}$ and $\boldsymbol{q}_{2}$, one needs the relevant parton-level cross sections, e.g. for gauge boson pair production, as a function of the relative transverse momentum $\boldsymbol{q}_{1}-\boldsymbol{q}_{2}$. Logarithms of the net transverse momentum $\boldsymbol{q}_{1}+\boldsymbol{q}_{2}$ are resummed by the exponential in (2.11).

Perturbative ingredients in the cross section are the anomalous dimensions $\gamma_{K}, \gamma_{a}$, the Collins-Soper kernel ${ }^{1} K$, the short-distance (or matching) coefficients ${ }^{1} C$, and finally the coefficient $C_{H}^{2}$ of the hard-scattering cross section, defined as $\hat{\sigma}$ divided by its treelevel value. ${ }^{13}$ In table 3 we specify different levels of accuracy, following the scheme of $[110,111]$. After expanding $\alpha_{s}\left(\mu^{\prime}\right)$ in $\gamma_{a}\left(\mu^{\prime}\right)$ and $\gamma_{K}\left(\mu^{\prime}\right)$ around $\alpha_{s}(\mu)$, the integral over $\mu^{\prime}$ in (2.11) gives powers of $\log \left(\mu / \mu_{0}\right)$. We thus find that $\gamma_{K}$ goes with double $\log$ arithms $\alpha_{s}(Q) \log ^{2}\left(Q / q_{T}\right)$, whilst $\gamma_{a}$ and ${ }^{1} K$ go with single logarithms $\alpha_{s}(Q) \log \left(Q / q_{T}\right)$. One therefore requires one order higher for $\gamma_{K}$ than for $\gamma_{a}$ and ${ }^{1} K$ (see table 3). Likewise, one finds that one needs the same perturbative order for the $\beta$ function as for $\gamma_{K}$. At accuracy $\mathrm{N}^{k} \mathrm{LO}$ one then has control over all terms from $\alpha_{s}^{n} \log ^{n+1}$ to $\alpha_{s}^{n+k} \log ^{n+1}$ in the exponent. $\gamma_{K}, \gamma_{a}$ and ${ }^{1} K$ all start at $\mathcal{O}\left(\alpha_{s}\right)$ and are known up to $\mathcal{O}\left(\alpha_{s}^{3}\right)$, both in the quark and in the gluon channel. A compilation of these results can for instance be found in appendix D of [99]. The notation in that work is related to the one we are using by

$$
\gamma_{K, a}=2 \Gamma_{\text {cusp }}^{a}, \quad \gamma_{a}=-\gamma_{V}^{a}, \quad \gamma_{F, a}(\mu, \zeta)=\gamma^{a}(\mu, \zeta)
$$

and

$$
{ }^{1} K_{a}(\boldsymbol{z}, \mu)=-2 \mathcal{D}^{a}\left(\mu, \boldsymbol{b}_{T}\right) \quad \text { with } \boldsymbol{z}=\boldsymbol{b}_{T}
$$

where $a=q, g$ and it is understood that the strong coupling is taken at scale $\mu$. Results for the quark channel are also given in [68], where the same notation as here is used (except that the Collins-Soper kernel is denoted by $\tilde{K}$ instead of $K)$.

At lowest order one has ${ }^{1} C_{a b}=\delta_{a b} \delta(1-x)$ and $C_{H}^{2}=1$. These coefficients are not associated with the resummation of large logarithms, and conventionally one requires them at one order in $\alpha_{s}$ lower than $\gamma_{a}$. This corresponds to counting $\log \left(Q / q_{T}\right)$ as order $1 / \alpha_{s}$.

\footnotetext{
${ }^{13}$ For definiteness we include the colour-singlet labels on $K$ and $C$ here, although this is the only colour channel that appears in SPS.
} 


\begin{tabular}{|ccccccc|}
\hline & $\gamma_{K}$ & $\gamma_{a}$ & ${ }^{R} K,{ }^{R R^{\prime}} M$ & ${ }^{R} C,{ }^{R R^{\prime}} C_{\text {int }}$ & $C_{H}^{2}$ & ${ }^{R} P$ \\
\hline $\mathrm{LL}$ & $\alpha_{s}$ & - & - & $\alpha_{s}^{0}$ & $\alpha_{s}^{0}$ & $\alpha_{s}$ \\
$\mathrm{NLL}$ & $\alpha_{s}^{2}$ & $\alpha_{s}$ & $\alpha_{s}$ & $\alpha_{s}^{0}$ & $\alpha_{s}^{0}$ & $\alpha_{s}$ \\
$\mathrm{NLL}^{\prime}$ & $\alpha_{s}^{2}$ & $\alpha_{s}$ & $\alpha_{s}$ & $\alpha_{s}$ & $\alpha_{s}$ & $\alpha_{s}^{2}$ \\
$\mathrm{NNLL}^{2}$ & $\alpha_{s}^{3}$ & $\alpha_{s}^{2}$ & $\alpha_{s}^{2}$ & $\alpha_{s}$ & $\alpha_{s}$ & $\alpha_{s}^{2}$ \\
$\mathrm{NNLL}^{\prime}$ & $\alpha_{s}^{3}$ & $\alpha_{s}^{2}$ & $\alpha_{s}^{2}$ & $\alpha_{s}^{2}$ & $\alpha_{s}^{2}$ & $\alpha_{s}^{3}$ \\
\hline
\end{tabular}

Table 3. Different levels of accuracy and the associated orders of $\alpha_{s}$ for the perturbative ingredients in the SPS cross section. The order required for the $\beta$ function is the same as for $\gamma_{K}$.

Taking them at the same order as $\gamma_{a}$ is indicated by a prime in the table. For unpolarised quarks or gluons, ${ }^{1} C$ has been computed up to $\mathcal{O}\left(\alpha_{s}^{2}\right)$ in [99] and [112]. With unpolarised protons this is sufficient for $q \bar{q}$ annihilation processes, whereas for $g g$ initiated processes one also requires the coefficient ${ }^{1} C_{\delta g g}$ for linear gluon polarisation in the TMDs. This is well known from the process $g g \rightarrow H$ (see e.g. [85] and references therein).

To the best of our knowledge, the hard-scattering coefficient $C_{H}^{2}$ has not been given in the literature for final states relevant in our context, such as $W^{+} W^{-}, Z H, H H$, etc. It should however be possible to extract it from the virtual corrections to the relevant amplitudes, which are known up to $\mathcal{O}\left(\alpha_{s}^{2}\right)$ in some channels (for gauge boson pair production see e.g. $[113,114]$ and references therein). Without this additional effort, the SPS contribution to the cross section can currently be evaluated at NLL accuracy.

There is one more type of large logarithms hidden in (2.11), namely the logarithms of $q_{T} / \Lambda$ that are resummed by DGLAP evolution of the PDFs on the r.h.s., from a typical starting scale of order $\Lambda$ up to $\mu_{0} \sim 1 /|\boldsymbol{z}|$. A customary choice is to take the DGLAP kernels ${ }^{1} P$ at one power in $\alpha_{s}$ higher than the matching coefficients ${ }^{1} C$, as shown in the table. This amounts to using LO evolution together with LO matching coefficients, etc. The perturbative accuracy of ${ }^{1} C$ is then retained even if $\log \left(q_{T} / \Lambda\right)$ is as large as $1 / \alpha_{s}$. For unpolarised PDFs, the DGLAP kernels are known up to $\mathcal{O}\left(\alpha_{s}^{3}\right)[115,116]$.

Interference between SPS and DPS. The perturbative ingredients required for computing the SPS/DPS interference term, as laid out in section 6.5, are the Collins-Soper kernels $K_{\alpha_{1} \alpha_{2} \mid \alpha_{0}}$ for twist-three TMDs, the splitting kernels $U$ appearing in their shortdistance approximation, and the interference between SPS and DPS hard-scattering amplitudes for the process under investigation. None of these have been calculated so far. It is easy to compute the $\mathcal{O}\left(\alpha_{s}\right)$ expressions of $K_{\alpha_{1} \alpha_{2} \mid \alpha_{0}}$ and $U$ by adapting the corresponding calculations for DPDs in [27]. Combining the known hard-scattering amplitudes for the relevant SPS and DPS processes is a straightforward exercise (but can be tedious, especially for heavy gauge bosons due to their three helicities).

DPS: small-y contribution. Let us now discuss the perturbative ingredients in the small-y expression (6.47) for DPS. $\gamma_{K}, \gamma_{a}$ and ${ }^{1} K$ are the same as in the SPS formula, and in addition one needs the matrix $R R^{\prime} M$, which starts at $\mathcal{O}\left(\alpha_{s}\right)$ and goes with single logarithms $\log \left(Q / q_{T}\right)$. The expression for the matching ${ }^{R} F_{\text {spl }}$ on PDFs involves a coefficient ${ }^{R} C_{\text {spl }}$, and 
the matching of ${ }^{R} F_{\text {int }}$ on twist-four distributions involves a coefficient ${ }^{R R^{\prime}} C_{\text {int }}$. The lowest order of $C_{\mathrm{spl}}$ and $C_{\mathrm{int}}$ is $\mathcal{O}\left(\alpha_{s}\right)$ and $\mathcal{O}\left(\alpha_{s}^{0}\right)$, respectively - their explicit definitions are not needed here. The hard-scattering subprocesses for DPS are simpler than for SPS with the same final state. For Drell-Yan and for Higgs boson production the hard-scattering coefficients $C_{H}^{2}$ are known up to $\mathcal{O}\left(\alpha_{s}^{3}\right)$. They can for instance be found in [68] for Drell-Yan production and in [117] for both channels.

The evolution kernel ${ }^{R R^{\prime}} M$ is known at $\mathcal{O}\left(\alpha_{s}^{2}\right)$ in the two-quark channel [74]; its $\mathcal{O}\left(\alpha_{s}\right)$ expressions for all channels are given in section 7.2. The matching coefficients ${ }^{R} C_{\mathrm{spl}}$ have been calculated at $\mathcal{O}\left(\alpha_{s}\right)$ in [27], and the related kernels ${ }^{R} T$ are compiled in section 7.4 here. For ${ }^{R} R^{\prime} C_{\text {int }}$, only the trivial order $\alpha_{s}^{0}$ is currently known, which corresponds to matching $F_{\text {int }}=G$. The kernels for the DGLAP evolution of the distributions $G$ are known at $\mathcal{O}\left(\alpha_{s}\right)$ and given in [118]; for a more general discussion of twist-four evolution we refer to [119]. At a given level of accuracy, their order should be the same as the one of ${ }^{R} P$ in table 3 .

When denoting the level of perturbative accuracy, one might think of combining equal orders in $\alpha_{s}$ for $C_{\mathrm{spl}}$ and $C_{\mathrm{int}}$. On the other hand, $C_{\mathrm{spl}}$ starts one order higher in $\alpha_{s}$ than $C_{\text {int }}$, and moreover the different combinations of $F_{\mathrm{spl}}$ and $F_{\text {int }}$ in the cross section have different power behaviour in $\Lambda^{2} / q_{T}^{2}$ according to (6.40). We thus find it more natural to take one power in $\alpha_{s}$ more for $C_{\mathrm{spl}}$ than for $C_{\mathrm{int}}$. With this naming convention, the small- $y$ expression of DPS can currently be evaluated to NLL accuracy. Missing ingredients for achieving NNLL in pure quark channels or NLL' in channels with gluons are the matching coefficients $C_{\text {spl }}$ and $C_{\text {int }}$ for DTMDs, as well as the two-loop DGLAP kernels for twist-four evolution.

DPS: large- $\boldsymbol{y}$ contribution. We now turn our attention to the large- $y$ expression (6.24) of DPS. The quantities $\gamma_{K}, \gamma_{a}$ and $C_{H}^{2}$ are the same as in the small- $y$ expressions of DPS. The resummation of single $\operatorname{logarithms} \log \left(Q / q_{T}\right)$ requires the Collins-Soper kernels ${ }^{R} K$ in the different colour channels, which can be obtained from the perturbative expression of $R R^{\prime} M\left(\boldsymbol{z}_{i}, \boldsymbol{y}\right)$ by taking the limit $\left|\boldsymbol{z}_{i}\right| \ll|\boldsymbol{y}|$ and using that in this limit one has the structure (6.16). In the two-quark channel one can thus obtain the $\mathcal{O}\left(\alpha_{s}^{2}\right)$ expression for ${ }^{R} K$ from the results of [74]; in all other channels one has the one-loop expressions, which we list in section 7.2. For the matching coefficients ${ }^{R} C$ we will derive $\mathcal{O}\left(\alpha_{s}\right)$ results in all colour and polarisation channels in the next section; most of them have been given in the literature before. One can thus evaluate gauge boson pair production with NNLL accuracy, whereas for DPS processes with one or two Higgs bosons in the final state, NLL' accuracy is currently achievable.

Let us finally take a closer look at the evolution of the DPDFs, which generates logarithms involving a hadronic starting scale $\Lambda$. The situation is now more involved than the one discussed for SPS above. In analogy to usual DGLAP evolution, there are single $\operatorname{logarithms} \log \left(q_{T} / \Lambda\right)$, whose resummation requires the $\zeta$ independent part of the evolution kernels ${ }^{R} P$. We will give $\mathcal{O}\left(\alpha_{s}\right)$ expressions for these kernels in all polarisation channels in section 7.3.5. The $\zeta$ dependence of the DPDFs, which has no counterpart for ordinary parton distributions, is made explicit in (6.27). Expanding $\alpha_{s}(\mu)$ in the anomalous dimension ${ }^{R} \gamma_{J}(\mu)$ around a fixed scale, we can perform the integral and obtain double logarithms $\log ^{2}(Q / \Lambda)-\log ^{2}\left(Q / q_{T}\right)=\log \left(\Lambda q_{T} / Q^{2}\right) \log \left(\Lambda / q_{T}\right)$ in the exponential. If both $\log \left(Q / q_{T}\right)$ 
and $\log \left(q_{T} / \Lambda\right)$ are counted as order $1 / \alpha_{s}$, one therefore needs ${ }^{R} \gamma_{J}$ at the same order as the cusp anomalous dimension $\gamma_{K}$. For smaller values of $\log \left(q_{T} / \Lambda\right)$ one may instead be content with ${ }^{R} \gamma_{J}$ at the same order as $\gamma_{a}$ and ${ }^{R} K$. From the two-loop results in [74] one can extract the $\mathcal{O}\left(\alpha_{s}^{2}\right)$ term of ${ }^{8} \gamma_{J}$, whereas for higher colour representations we currently only know the $\mathcal{O}\left(\alpha_{s}\right)$ results given in section 7.2. We note that in (6.27) there are also single logarithms $\log \left(Q / q_{T}\right)$ multiplied with ${ }^{R} J(\boldsymbol{y})$, which at large $\boldsymbol{y}$ is beyond the control of perturbation theory.

\section{One-loop results}

In this section, we present the perturbative ingredients necessary for evaluating DPS at NLL accuracy (see table 3). Some of the expressions are quoted from the literature to make the presentation self-contained, whereas for evolution kernels and matching coefficients we verify previous and derive new results.

In section 7.1 we discuss the hard-scattering cross sections for Drell-Yan and for Higgs production via gluon fusion, and in section 7.2 we give the one-loop expressions of the Collins-Soper kernels ${ }^{R R^{\prime}} K_{a_{1} a_{2}}$ for all parton and colour channels. Section 7.3 is devoted to the $\mathcal{O}\left(\alpha_{s}\right)$ matching coefficients ${ }^{R} C_{a b}$ of DTMDs in the large- $\boldsymbol{y}$ region. After explaining a number of computational details, we present results for all polarisation and colour combinations in sections 7.3.4 and 7.3.5. As a corollary we obtain the one-loop DGLAP kernels (7.91) for colour non-singlet DPDFs. In section 7.4 we list the $\mathcal{O}\left(\alpha_{s}\right)$ splitting kernels ${ }^{R} T_{a_{0} \rightarrow a_{1} a_{2}}$ of DTMDs in the small- $\boldsymbol{y}$ region, adapting the results of [27] to the notation used here.

\subsection{TMD hard-scattering cross sections}

We define the coefficients $C_{H}^{2}$ of the hard-scattering cross section as

$$
\hat{\sigma}\left(Q^{2}, \mu^{2}\right)=\hat{\sigma}_{0}\left(Q^{2}\right) C_{H}^{2}\left(Q^{2}, \mu^{2}\right)
$$

for a given partonic channel, where $\hat{\sigma}_{0}$ is the cross section at lowest order in $\alpha_{s}$. The expressions for $\hat{\sigma}_{0}$ can be found in many places, for instance in [44] for the production of an electroweak gauge boson $V$ and its subsequent decay into a lepton pair, and in [85] for Higgs production via $g g$ fusion in the limit of large top mass. The hard-scattering coefficients read

$$
\begin{aligned}
& C_{H, q \bar{q} \rightarrow V}^{2}\left(Q^{2}, \mu^{2}\right)=1+\frac{\alpha_{s}(\mu)}{2 \pi} C_{F}\left[-\ln ^{2} \frac{Q^{2}}{\mu^{2}}+3 \ln \frac{Q^{2}}{\mu^{2}}-8+\frac{7 \pi^{2}}{6}\right], \\
& C_{H, g g \rightarrow H}^{2}\left(Q^{2}, \mu^{2}\right)=\left(1+\frac{\alpha_{s}(\mu)}{2 \pi} C_{A}\left[-\ln ^{2} \frac{Q^{2}}{\mu^{2}}+\frac{7 \pi^{2}}{6}\right]\right)\left(1+\frac{\alpha_{s}(\mu)}{2 \pi}\left[5 C_{A}-3 C_{F}\right]\right)
\end{aligned}
$$

and are known up to $\mathcal{O}\left(\alpha_{s}^{3}\right)$, see for instance appendix A of [117]. The colour factors are $C_{F}=\left(N^{2}-1\right) /(2 N)$ and $C_{A}=N$ as usual. The first factor in $C_{H, g g \rightarrow H}^{2}$ is for two-gluon fusion via the local current $F^{\mu \nu a} F_{\mu \nu}^{a}$ and the second factor for the coupling of this current to the Higgs boson via a top quark loop. Throughout this section we drop the explicit 
indication of higher-order corrections in equations, omitting a term $+\mathcal{O}\left(\alpha_{s}^{2}\right)$ in the first line of (7.2) and so forth.

An important property of the coefficients $C_{H}^{2}$ for Drell-Yan and Higgs production is their independence of the incoming parton polarisations. This holds at all orders in $\alpha_{s}$. For $g g \rightarrow H$ it follows from the fact that angular momentum conservation only allows for two non-vanishing helicity amplitudes, which are related by parity invariance. Therefore, all $\alpha_{s}$ corrections can be expressed by a single hard-scattering coefficient. The argument holds for the production of any scalar or pseudoscalar boson via $g g$ fusion.

For $q \bar{q}$ annihilation into a boson via the vector current, one additionally needs the conservation of quark chirality, which together with angular momentum and parity conservation leaves a single independent helicity amplitude. To appeal to quark chirality conservation in a calculation using dimensional regularisation, one can adapt the argument given in [120]. As long as one works in $D=4-2 \epsilon$ dimensions, one can avoid specifying the polarisation of the incoming partons and instead compute the hard scattering as a matrix in Dirac space. Chirality conservation then technically means that the hard-scattering amplitude contains an odd number of $\gamma_{\mu}$ matrices at any perturbative order. The same holds for the terms that must be subtracted to remove ultraviolet and infrared divergences. After these subtractions have been done, one can take $D=4$ and use the relation between quark helicity and chirality.

The extension of this argument to $q \bar{q}$ annihilation via the axial vector current is simple in $D=4$ dimensions: anticommuting the $\gamma_{5}$ matrix from the current, one finds $A_{\mu}=V_{\mu} \gamma_{5}$ for the Dirac matrix of the hard-scattering amplitudes of the two currents (with their divergences subtracted). Their independent helicity amplitudes are hence the same (or opposite in sign). To complete the argument, one needs to discuss the implementation of the electroweak axial current in dimensional regularisation, which we shall not do here.

\subsection{Collins-Soper kernels and anomalous dimensions}

In the limit of small $\boldsymbol{z}_{1}, \boldsymbol{z}_{1}$ and $\boldsymbol{y}$, the soft matrix for DPS can be directly computed in perturbation theory, as we derived in (6.36). Setting $U=\mathbb{1}$ in (A.12), we simply have $K=\widehat{K}=d S / d Y$ at $\mathcal{O}\left(\alpha_{s}\right)$. The one-loop calculation of $S$ in the $q q$ channel is presented in detail in section 3.3.2 of [27]. We can rewrite the results there in such a way that we have a separate dependence on the two scales $\mu_{1}$ and $\mu_{2}$. Splitting off a diagonal part according to $(5.16)$, we get

$$
K_{q q}=C_{F}\left[K\left(\boldsymbol{z}_{1} ; \mu_{1}\right)+K\left(\boldsymbol{z}_{2} ; \mu_{2}\right)\right]\left(\begin{array}{cc}
1 & 0 \\
0 & 1
\end{array}\right)+M_{q q}
$$

with

$$
M_{q q}=\left(\begin{array}{cc}
0 & \frac{\sqrt{N^{2}-1}}{2 N} K_{d} \\
\frac{\sqrt{N^{2}-1}}{2 N} K_{d} & -\frac{N}{2} K_{y}-\frac{1}{N} K_{d}
\end{array}\right)
$$


where the matrices are in colour representation space, with $R=(1,8)$. We recall that $M_{q q}$ is renormalisation scale independent. The scale independent combinations of kernels read

$$
\begin{aligned}
K_{y}\left(\boldsymbol{z}_{i}, \boldsymbol{y}\right)= & K\left(\boldsymbol{z}_{1} ; \mu\right)+K\left(\boldsymbol{z}_{2} ; \mu\right)-K\left(\boldsymbol{y}+\frac{1}{2}\left(\boldsymbol{z}_{1}+\boldsymbol{z}_{2}\right) ; \mu\right)-K\left(\boldsymbol{y}-\frac{1}{2}\left(\boldsymbol{z}_{1}+\boldsymbol{z}_{2}\right) ; \mu\right), \\
K_{d}\left(\boldsymbol{z}_{i}, \boldsymbol{y}\right)= & K\left(\boldsymbol{y}+\frac{1}{2}\left(\boldsymbol{z}_{1}+\boldsymbol{z}_{2}\right) ; \mu\right)+K\left(\boldsymbol{y}-\frac{1}{2}\left(\boldsymbol{z}_{1}+\boldsymbol{z}_{2}\right) ; \mu\right) \\
& -K\left(\boldsymbol{y}+\frac{1}{2}\left(\boldsymbol{z}_{1}-\boldsymbol{z}_{2}\right) ; \mu\right)-K\left(\boldsymbol{y}-\frac{1}{2}\left(\boldsymbol{z}_{1}-\boldsymbol{z}_{2}\right) ; \mu\right)
\end{aligned}
$$

where

$$
K(\boldsymbol{z} ; \mu)=-\frac{\alpha_{s}(\mu)}{\pi} \log \frac{\boldsymbol{z}^{2} \mu^{2}}{b_{0}^{2}}
$$

is the one-loop Collins-Soper kernel for single parton TMDs, with the colour factor removed. We recall that $b_{0}=2 e^{-\gamma_{E}}$. We note that the two-loop result in section 4 of [74] has the form given by (7.3), (7.4) and (7.5) if one replaces $C_{F} K(\boldsymbol{z} ; \mu)$ with the two-loop Collins-Soper kernel for a single quark TMD.

In the solution (5.17) of the evolution equations, one needs the matrix exponential of $M_{q q}$ times $L=\log \sqrt{\zeta / \zeta_{0}}$, which reads (see section 3.4.2 of [27])

$$
\exp \left[L M_{q q}\right]=\frac{1}{d_{+}-d_{-}}\left(\begin{array}{cc}
d_{+} e^{L d_{-}}-d_{-} e^{L d_{+}} & \frac{\sqrt{N^{2}-1}}{2 N} K_{d}\left(e^{L d_{+}}-e^{L d_{-}}\right) \\
\frac{\sqrt{N^{2}-1}}{2 N} K_{d}\left(e^{L d_{+}}-e^{L d_{-}}\right) & d_{+} e^{L d_{+}}-d_{-} e^{L d_{-}}
\end{array}\right)
$$

with

$$
d_{ \pm}=\frac{1}{2 N}\left[-\frac{N^{2}}{2} K_{y}-K_{d} \pm \sqrt{\left(\frac{N^{2}}{2} K_{y}+K_{d}\right)^{2}+\left(N^{2}-1\right) K_{d}^{2}}\right] .
$$

An interesting situation arises if $\left|K_{d}\right| \ll \sqrt{N^{2}-1}\left|K_{y}\right|$, which holds for instance in the large- $N$ limit. As shown in section 3.4.2 of [27], one then finds that ${ }^{8} F(\zeta)$ is suppressed compared with ${ }^{1} F(\zeta)$ for $\zeta \gg \zeta_{0}$. Note that the above condition always holds in the limit $\boldsymbol{z}_{1}=\boldsymbol{z}_{2}=\mathbf{0}$, where $K_{d}$ tends to zero.

It is easy to extend the above results to other parton channels, because the one-loop graphs remain the same up to colour factors. For the quark-gluon channels, we obtain

$$
K_{q g}\left(\boldsymbol{z}_{i}, \boldsymbol{y} ; \mu_{i}\right)=\left[C_{F} K\left(\boldsymbol{z}_{1} ; \mu_{1}\right)+C_{A} K\left(\boldsymbol{z}_{2} ; \mu_{2}\right)\right] \mathbb{1}+M_{q g}
$$

with

$$
M_{q g}=\left(\begin{array}{ccc}
0 & \frac{1}{\sqrt{2}} K_{d} & 0 \\
\frac{1}{\sqrt{2}} K_{d} & -\frac{N}{2}\left(K_{y}+\frac{1}{2} K_{d}\right) & \frac{\sqrt{N^{2}-4}}{4} K_{d} \\
0 & \frac{\sqrt{N^{2}-4}}{4} K_{d} & -\frac{N}{2}\left(K_{y}+\frac{1}{2} K_{d}\right)
\end{array}\right) .
$$

Here the space of colour representations is $R=(1, A, S)$, corresponding to the projectors in (4.4), and $\mathbb{1}$ is the unit matrix in that space. For the two-gluon channel we get the kernel

$$
K_{g g}\left(\boldsymbol{z}_{i}, \boldsymbol{y} ; \mu_{i}\right)=C_{A}\left[K\left(\boldsymbol{z}_{1} ; \mu_{1}\right)+K\left(\boldsymbol{z}_{2} ; \mu_{2}\right)\right] \mathbb{1}+M_{g g}
$$


with

$$
M_{g g}=\left(\begin{array}{ccccc}
0 & \frac{N}{\sqrt{N^{2}-1}} K_{d} & 0 & 0 & 0 \\
\frac{N}{\sqrt{N^{2}-1}} K_{d} & -\frac{N}{2}\left(K_{y}+\frac{1}{2} K_{d}\right) & \frac{N}{4} K_{d} & 0 & \sqrt{\frac{3}{8}} K_{d} \\
0 & \frac{N}{4} K_{d} & -\frac{N}{2}\left(K_{y}+\frac{1}{2} K_{d}\right) & -\frac{3}{\sqrt{10}} K_{d} & 0 \\
0 & 0 & -\frac{3}{\sqrt{10}} K_{d} & -3\left(K_{y}+\frac{1}{2} K_{d}\right) & -\sqrt{\frac{3}{5}} K_{d} \\
0 & \sqrt{\frac{3}{8}} K_{d} & 0 & -\sqrt{\frac{3}{5}} K_{d} & -4\left(K_{y}+\frac{1}{2} K_{d}\right)
\end{array}\right)
$$

in the space of colour representations $R=(1, A, S, D, 27)$, corresponding to the projectors in (4.3). In the rows and columns for $D$ and 27, we have given the numerical coefficients for $N=3$. The Collins-Soper kernels in channels with antiquarks can be obtained from the above expressions by using the relation (4.29) and the analogue of (4.31) for $K_{a_{1} a_{2}}$.

The matrices $M_{q g}$ and $M_{g g}$ are non-singular for generic values of the functions $K_{y}$ and $K_{d}$. The eigenvalues of $M_{q g}$ can be calculated analytically, but the expressions are rather lengthy and we refrain from giving them here. For $N=3$, the eigenvalues of $M_{g g}$ read

$$
-3\left(K_{y}+\frac{1}{2} K_{d}\right), \quad-\frac{3}{2} K_{y}, \quad-\frac{3}{2}\left(K_{y}+K_{d}\right), \quad-\left(2 K_{y}+K_{d}\right) \pm \sqrt{\left(2 K_{y}+K_{d}\right)^{2}+3 K_{d}^{2}} .
$$

\subsubsection{Limit of small $z_{1}$ and $z_{2}$}

We now consider the limit where $\left|\boldsymbol{z}_{1}\right|,\left|\boldsymbol{z}_{2}\right| \ll|\boldsymbol{y}|$. Expanding (7.3), (7.9) and (7.11) in powers of $\left|\boldsymbol{z}_{1}\right| /|\boldsymbol{y}|$ and $\left|\boldsymbol{z}_{2}\right| /|\boldsymbol{y}|$ and comparing the leading terms with the structure in (6.16), where the full kernel ${ }^{R R^{\prime}} K_{a_{1} a_{2}}\left(\boldsymbol{z}_{i}, \boldsymbol{y} ; \mu_{i}\right)$ is split into three separate contributions, we can deduce the LO expressions of the kernels ${ }^{R} K_{a}$ and ${ }^{R} J$. We obtain

$$
\begin{aligned}
{ }^{1} K_{q}(\boldsymbol{z} ; \mu) & =C_{F} K(\boldsymbol{z} ; \mu), & { }^{8} K_{q}(\boldsymbol{z} ; \mu) & =-\frac{1}{2 N} K(\boldsymbol{z} ; \mu), \\
{ }^{1} K_{g}(\boldsymbol{z} ; \mu) & =C_{A} K(\boldsymbol{z} ; \mu), & { }^{A} K_{g}(\boldsymbol{z} ; \mu) & ={ }^{S} K_{g}(\boldsymbol{z} ; \mu)=\frac{N}{2} K(\boldsymbol{z} ; \mu)
\end{aligned}
$$

and

$$
{ }^{1} J=0, \quad{ }^{8} J\left(\boldsymbol{y} ; \mu_{i}\right)=\frac{N}{2}\left[K\left(\boldsymbol{y} ; \mu_{1}\right)+K\left(\boldsymbol{y} ; \mu_{2}\right)\right]
$$

with $K(\boldsymbol{z} ; \mu)$ defined in (7.6). For the higher gluon representations we find

$$
{ }^{D} K_{g}(\boldsymbol{z} ; \mu)=0, \quad{ }^{27} K_{g}(\boldsymbol{z} ; \mu)=-K(\boldsymbol{z} ; \mu)
$$

and

$$
{ }^{D} J\left(\boldsymbol{z} ; \mu_{i}\right)=3\left[K\left(\boldsymbol{y} ; \mu_{1}\right)+K\left(\boldsymbol{y} ; \mu_{2}\right)\right], \quad{ }^{27} J\left(\boldsymbol{z} ; \mu_{i}\right)=4\left[K\left(\boldsymbol{y} ; \mu_{1}\right)+K\left(\boldsymbol{y} ; \mu_{2}\right)\right],
$$


where again we have set $N=3$. The expressions for $J$ are of course only valid at perturbatively small $\boldsymbol{y}$.

Using the explicit form of $K(\boldsymbol{z} ; \mu)$, we obtain anomalous dimensions

$$
\begin{aligned}
{ }^{1} \gamma_{K, q} & =2 C_{F} \frac{\alpha_{s}}{\pi}, & { }^{8} \gamma_{K, q} & =-\frac{1}{N} \frac{\alpha_{s}}{\pi}, \\
{ }^{1} \gamma_{J} & =0, & { }^{8} \gamma_{J} & =N \frac{\alpha_{s}}{\pi} .
\end{aligned}
$$

The expressions for other colour representations are readily obtained from (7.14) and (7.15). We note that ${ }^{8} \gamma_{K, q}$ and ${ }^{27} \gamma_{K, g}$ are negative. Furthermore, we find that ${ }^{R} \gamma_{J}>0$ for all $R \neq 1$.

For completeness we also give the anomalous dimensions $\gamma_{a}$, which can be taken from the literature for single TMDs, e.g. from [99] (see our equation (6.66) for notation). The one-loop expressions are

$$
\gamma_{q}=\frac{3}{2} C_{F} \frac{\alpha_{s}}{\pi}, \quad \gamma_{g}=\frac{\beta_{0}}{2} \frac{\alpha_{s}}{\pi}
$$

with $\beta_{0}=\frac{11}{3} C_{A}-\frac{2}{3} n_{F}$. Finally, one has

$$
\begin{aligned}
& \gamma_{K, q}=2 C_{F} \frac{\alpha_{s}}{\pi}+\frac{C_{F}}{2}\left(\frac{67-3 \pi^{2}}{9} C_{A}-\frac{10}{9} n_{F}\right)\left(\frac{\alpha_{s}}{\pi}\right)^{2}, \\
& \gamma_{K, g}=\frac{C_{A}}{C_{F}} \gamma_{K, q}
\end{aligned}
$$

for the cusp anomalous dimension at two-loop accuracy. Using the all-order relation

$$
{ }^{8} \gamma_{J}=\frac{1}{2} \gamma_{K, g},
$$

which follows from (6.30), we readily get the two-loop expression of ${ }^{8} \gamma_{J}$ as well.

\subsection{Matching coefficients for DTMDs}

We now turn to the short-distance coefficients for matching DTMDs on DPDFs in the large- $\boldsymbol{y}$ regime. In the colour singlet channel, they are equal to the coefficients for matching TMDs on PDFs, which for most polarisation combinations have been computed by several groups $[65,66,85,121,122]$. In addition to extending these results to colour non-singlet channels, we have computed the colour singlet coefficients for all polarisation channels. In the following two subsections, we describe in detail our method, which allows us to compute the one-loop matching coefficients from real emission graphs alone and to handle gluon polarisation without using the Levi-Civita tensor in $D=4-2 \epsilon$ dimensions. Subtleties of renormalisation in both the quark and gluon sector are discussed in section 7.3.3. We give our final results for the colour singlet coefficients in section 7.3.4 and explain their extension to other colour channels in section 7.3.5. 


\subsubsection{General procedure}

To compute the matching coefficients, we take matrix elements of the operator relations in (6.5) and (6.6), using either the vacuum or a single parton as external states. We define

$$
\begin{aligned}
2 \pi \delta\left(p^{+}-p^{\prime}\right) 2 p^{+R} \mathcal{M}_{a b}(x, \boldsymbol{z}) & =\frac{1}{\mathcal{N}_{b}} \frac{1}{m(R)}\left\langle b, p^{\prime},\left.r^{\prime}\right|^{R} O_{a}^{\underline{r}}(x, \boldsymbol{y}, \boldsymbol{z}) \mid b, p, r\right\rangle \\
& =\varepsilon_{a}(R) \frac{\mathcal{N}_{a}}{\mathcal{N}_{b}} \frac{P_{\underline{R}}^{\underline{r}}}{m(R)}\left\langle b, p^{\prime}, r^{\prime}\left|O \frac{s}{a}(x, \boldsymbol{y}, \boldsymbol{z})\right| b, p, r\right\rangle, \\
{ }^{R} \mathcal{M}_{S, a}(\boldsymbol{z}) & =\frac{P_{\bar{r}}^{\underline{s}}}{m(R)}\left\langle 0\left|O O \frac{\underline{r}}{S}, \frac{s}{a}(\boldsymbol{y}, \boldsymbol{z})\right| 0\right\rangle,
\end{aligned}
$$

where $|b, p, r\rangle$ is a parton state $b$ with momentum $p$ and colour index $r$, and the colour projected operator ${ }^{R} O \underline{a}$ was introduced in (4.18). We recall that $\underline{r}=\left(r, r^{\prime}\right)$ and that repeated colour indices are summed over. We gloss over the issue of parton polarisation for the time being. In the colour singlet sector, one has $\varepsilon_{a}(1) / m(1)=1$ and the contraction with $P_{1}^{\underline{r}} \underline{\underline{s}} \mathcal{N}_{a} / \mathcal{N}_{b}$ in ${ }^{1} \mathcal{M}_{a b}$ implies a sum over the colour indices of the operator and an average over the colour of the parton state. Thus, ${ }^{1} \mathcal{M}_{a b}(x, z)$ is the unsubtracted TMD for parton $a$ in target $b$ according to (3.11). Likewise, ${ }^{1} \mathcal{M}_{S, a}(\boldsymbol{z})$ is the soft factor for single TMDs. In analogy to (7.22) we define matrix elements ${ }^{R} \mathcal{M}_{a b}(x)$ and ${ }^{R} \mathcal{M}_{S, a}$ without argument $\boldsymbol{z}$ from the operators with $\boldsymbol{z}=\mathbf{0}$; thus ${ }^{1} \mathcal{M}_{a b}(x)$ is the PDF for parton $a$ in target $b$. It is understood that all operators are renormalised, and we recall that the operators at $\boldsymbol{z}=\mathbf{0}$ require additional renormalisation compared with those at finite $\boldsymbol{z}$.

According to the operator matching equations (6.6) and (6.5), we thus have

$$
\begin{aligned}
{ }^{R} \mathcal{M}_{a c}(x, \boldsymbol{z}) & =\sum_{b}{ }^{R} C_{\mathrm{us}, a b}\left(x^{\prime}, \boldsymbol{z}\right) \underset{x}{\otimes}{ }^{R} \mathcal{M}_{b c}\left(x^{\prime}\right), \\
{ }^{R} \mathcal{M}_{S, a}(\boldsymbol{z}) & ={ }^{R} C_{S, a}(\boldsymbol{z})^{R} \mathcal{M}_{S, a} .
\end{aligned}
$$

We now expand the quantities in these relations up to $\mathcal{O}\left(\alpha_{s}\right)$, denoting the zeroth and first orders with superscripts (0) and (1), respectively. At tree level, the matrix elements $\left\langle b, p^{\prime}, r^{\prime}\left|O \frac{s}{a}\right| b, p, r\right\rangle$ and $\left\langle 0\left|O \frac{r}{S}, a,\right| 0\right\rangle$ have the colour structure $\delta_{r s} \delta_{r^{\prime} s^{\prime}}$, which gives

$$
{ }^{R} \mathcal{M}_{a b}^{(0)}(x, \boldsymbol{z})={ }^{R} \mathcal{M}_{a b}^{(0)}(x)=\delta_{a b} \delta(1-x), \quad{ }^{R} \mathcal{M}_{S, a}^{(0)}(\boldsymbol{z})={ }^{R} \mathcal{M}_{S, a}^{(0)}=1
$$

for the matrix elements on both sides of (7.23), which results in zeroth-order matching coefficients

$$
{ }^{R} C_{\mathrm{us}, a b}^{(0)}(x, \boldsymbol{z})=\delta_{a b} \delta(1-x), \quad{ }^{R} C_{S, a}^{(0)}(\boldsymbol{z})=1 .
$$

Inserting this into the $\mathcal{O}\left(\alpha_{s}\right)$ terms of (7.23), we obtain

$$
\begin{aligned}
{ }^{R} C_{\mathrm{us}, a b}^{(1)}(x, \boldsymbol{z}) & ={ }^{R} \mathcal{M}_{a b}^{(1)}(x, \boldsymbol{z})-{ }^{R} \mathcal{M}_{a b}^{(1)}(x), \\
{ }^{R} C_{S, a}^{(1)}(\boldsymbol{z}) & ={ }^{R} \mathcal{M}_{S, a}^{(1)}(\boldsymbol{z})-{ }^{R} \mathcal{M}_{S, a}^{(1)} .
\end{aligned}
$$

The one-loop matching coefficients are thus given as the difference of $\mathcal{O}\left(\alpha_{s}\right)$ matrix elements at finite and at zero $\boldsymbol{z}$. Virtual graphs cancel in this difference, because they do not depend on $\boldsymbol{z}$, so we can limit our calculation to real graphs. 
To obtain the matching coefficient ${ }^{R} C_{a b}$ of the TMD, we need the square root of ${ }^{R} C_{S, a}$ according to (6.10). At $\mathcal{O}\left(\alpha_{s}\right)$, we have

$$
\sqrt{{ }^{R} C_{S, a}^{(1)}(\boldsymbol{z} ; 2 Y)}=1+\frac{1}{2}{ }^{R} C_{S, a}^{(1)}(\boldsymbol{z} ; 2 Y)=1+{ }^{R} C_{S, a}^{(1)}(\boldsymbol{z} ; Y) .
$$

In the second step, we have used that ${ }^{R} C_{S, a}^{(1)}(\boldsymbol{z} ; Y)$ is linear in $Y$, which readily follows from its evolution equation (6.14) and the fact that ${ }^{R} K_{a}=\mathcal{O}\left(\alpha_{s}\right)$. Inserting this into (6.10), we thus obtain at $\mathcal{O}\left(\alpha_{s}\right)$

$$
\begin{aligned}
{ }^{R} C_{a b}(x, \boldsymbol{z} ; \zeta)= & \delta_{a b} \delta(1-x) \\
& +\lim _{Y_{L} \rightarrow-\infty}\left[{ }^{R} C_{\mathrm{us}, a b}^{(1)}\left(x, \boldsymbol{z} ; Y_{L}\right)-\delta_{a b} \delta(1-x){ }^{R} C_{S, a}^{(1)}\left(\boldsymbol{z} ; Y_{C}-Y_{L}\right)\right],
\end{aligned}
$$

where in the present section we use the definition (3.50) of $\zeta$, which refers to a single parton with plus-momentum $x p^{+}$. We have omitted the argument $\mu$ in all functions for brevity.

\subsubsection{Calculation of gluon-gluon matching coefficients}

We now describe in detail our procedure to compute the one-loop matching coefficients in the pure gluon channel. Throughout this subsection, we consider the colour singlet sector, $R=1$, and drop the corresponding index. In turn, we now make gluon polarisation explicit. We take gluon operators $O_{g}^{j j^{\prime}}(x, \boldsymbol{y}, \boldsymbol{z})$ with open polarisation indices, obtained from (3.10) and (3.6) by dropping the projection operator $\Pi_{a}^{j j^{\prime}}$ in the latter equation. We also take open polarisation indices $i, i^{\prime}$ for the gluons in the corresponding matrix element (7.22), which thus carries four Lorentz indices, $\mathcal{M}_{g g}^{j j^{\prime}, i i^{\prime}}$. All of them are restricted to be in the $D-2$ transverse dimensions. The subscripts $g$ refer of course only to the parton type here, and not to its polarisation. For the gluon matrix elements, we thus have

$$
\mathcal{M}_{g g}^{j j^{\prime}, l l^{\prime}}(x, \boldsymbol{z})=C_{\mathrm{us}, g g}^{j j^{\prime}, i i^{\prime}}\left(x^{\prime}, \boldsymbol{z}\right) \underset{x}{\otimes} \mathcal{M}_{g g}^{i i^{\prime}, l l^{\prime}}\left(x^{\prime}\right)+\{\text { quark-gluon mixing terms }\}
$$

and

$$
\mathcal{M}_{g g}^{(0) j j^{\prime}, i i}(x, \boldsymbol{z})=\mathcal{M}_{g g}^{(0) j j^{\prime}, i i^{\prime}}(x)=C_{g g}^{(0)} j j^{\prime}, i i(x, \boldsymbol{z})=\delta^{i j} \delta^{i^{\prime} j^{\prime}} \delta(1-x),
$$

which is combined with the matching of the soft factor into

$$
C_{g g}^{(1) j j^{\prime}, i i}(x, \boldsymbol{z} ; \zeta)=\lim _{Y_{L} \rightarrow-\infty}\left[C_{\mathrm{us}, g g}^{(1) j j^{\prime}, i i}\left(x, \boldsymbol{z} ; Y_{L}\right)-\delta^{i j} \delta^{i^{\prime} j^{\prime}} \delta(1-x) C_{S, a}^{(1)}\left(\boldsymbol{z} ; Y_{C}-Y_{L}\right)\right] .
$$

The virtual graphs contributing to the one-loop matrix elements are shown in figure 10. They are independent of $\boldsymbol{z}$ (the Fourier conjugated distance to the transverse momentum carried by partons or eikonal lines) and hence cancel in the matching coefficient. Therefore we only compute the real graphs in figure 11 . We omit so-called Wilson line self interactions, where a gluon is exchanged between eikonal lines along the same direction $v$, referring to chapter 13.7 of [65] for further discussion.

We work in Feynman gauge and compute cut graphs (where the emitted gluon is explicitly put on shell), using the Feynman rules given in appendix D. For graph 11b, we 


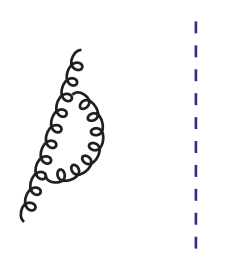

(a)

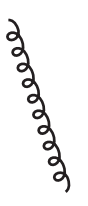

\$

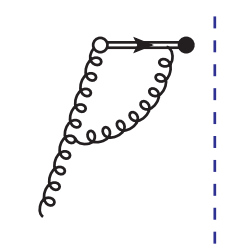

(b)

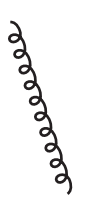

\$

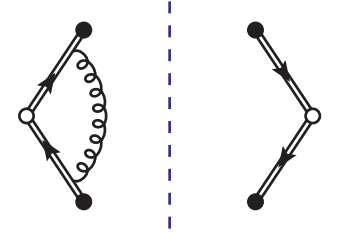

(c)

Figure 10. Virtual graphs contributing to the matrix elements $\mathcal{M}_{g g}^{(1) j j^{\prime}, i i}$ (a and b) and $\mathcal{M}_{S, g}^{(1)}$ (c). Not shown are complex conjugate graphs and the analogue of (a) with a quark instead of a gluon loop. All eikonal lines are in the adjoint representation.

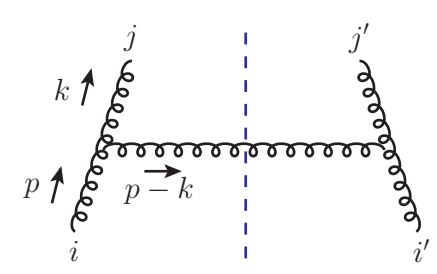

(a)

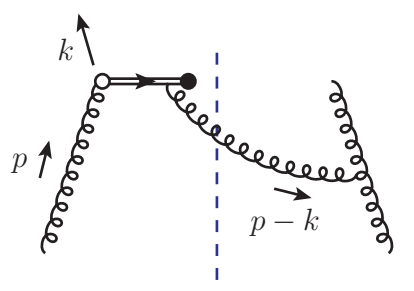

(b)

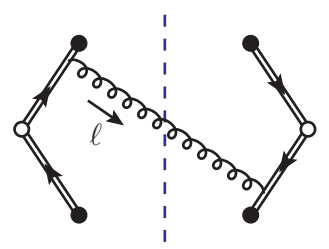

(c)

Figure 11. Real graphs contributing to the matrix elements $\mathcal{M}_{g g}^{(1) j j^{\prime}, i i}$ (a and b) and $\mathcal{M}_{S, g}^{(1)}$ (c). Not shown are the complex conjugates of graphs (b) and (c).

then obtain

$$
\begin{aligned}
& \left.C_{\mathrm{us}, g g}^{(1) j j^{\prime}, i i^{\prime}}\left(x, \boldsymbol{z} ; Y_{L} \rightarrow-\infty\right)\right|_{\text {figure } 11 \mathrm{~b}}=\frac{1}{x p^{+}} \int \frac{d^{4-2 \epsilon} k}{(2 \pi)^{4-2 \epsilon}}\left(e^{i \boldsymbol{k} \boldsymbol{z}}-1\right) 2 \pi \delta\left((p-k)^{2}\right) \delta\left(k^{+}-x p^{+}\right) \\
& \quad \times \frac{\delta_{a a^{\prime}} \delta_{d e^{\prime}}}{N^{2}-1}\left(g \mu^{\epsilon} n^{\nu} f^{d b c}\right)\left(-g_{\nu \nu^{\prime}} \delta_{b b^{\prime}}\right) \frac{i \delta_{c^{\prime} d^{\prime}} g_{\rho^{\prime} \tau^{\prime}}}{k^{2}-i 0} \frac{i \delta_{c e}}{(p-k) n-i 0} \\
& \quad \times\left(g \mu^{\epsilon} f^{a^{\prime} b^{\prime} c^{\prime}}\left[(p-2 k)^{i^{\prime}} g^{\nu^{\prime} \rho^{\prime}}+(k+p)^{\nu^{\prime}} g^{i^{\prime} \rho^{\prime}}+(k-2 p)^{\rho^{\prime}} g^{i^{\prime} \nu^{\prime}}\right]\right) \\
& \quad \times\left(-i\left[k n g^{i j}-p^{j} n^{i}\right] \delta_{a e}\right)\left(i\left[k n g^{\tau^{\prime} j^{\prime}}-k^{j^{\prime}} n^{\tau^{\prime}}\right] \delta_{d^{\prime} e^{\prime}}\right)+\left.C_{\mathrm{us}, \epsilon}\right|_{\text {figure } 11 \mathrm{~b}},
\end{aligned}
$$

where $n$ is the four-vector with $n^{-}=1, n^{+}=0$ and $\boldsymbol{n}=\mathbf{0}$. The assignment of polarisation indices is shown in figure 11a. All primed indices refer to the right of the final-state cut, and $a, b, \ldots, e$ are colour octet indices. The factor $e^{i \boldsymbol{k} z}-1$ represents the difference between the matrix element for nonzero and zero $z$. In accordance with our choice of light-cone coordinates, the components $p^{-}$and $\boldsymbol{p}$ of the target momentum are zero. The factor $\left(x p^{+}\right)^{-1}$ comes from the definition (3.10) of the operator $O_{g}$. The ultraviolet counterterm $C_{\mathrm{us}, \epsilon}$ will be discussed below.

In (7.32), we have taken the limit $Y_{L} \rightarrow-\infty$ by setting $v_{L}=n$ in the eikonal propagator. (Only then can we use the Feynman rule given in figure 14). This gives a factor $p^{+}-k^{+}=p^{+}(1-x)$ in the denominator. The resulting rapidity divergences in the convolution of $C_{\mathrm{us}, g g}$ with a PDF or a DPDF are cancelled by corresponding divergences in the 
soft factor, as we shall see shortly. In this sense, one should understand the limit $Y_{L} \rightarrow-\infty$ in $C_{\mathrm{us}, g g}$ as being taken in the combination (7.31).

Adding the complex conjugate of (7.32) and the expression for graph 11a, we get

$$
\begin{aligned}
& C_{\mathrm{us}, g g}^{(1) j j^{\prime}, i i^{\prime}}\left(x, \boldsymbol{z} ; Y_{L} \rightarrow-\infty\right)=\frac{\alpha_{s} C_{A}}{\pi^{2}}(2 \pi \mu)^{2 \epsilon} \int \frac{d^{2-2 \epsilon} \boldsymbol{k}}{\boldsymbol{k}^{2}}\left(e^{i \boldsymbol{k} \boldsymbol{z}}-1\right) \\
& \times\left\{\left[\frac{x}{1-x}+\frac{(1-x)\left(1+x^{2}\right)}{x}\right] \frac{1}{2(1-\epsilon)} \delta^{i i^{\prime}} \delta^{j j^{\prime}}\right. \\
&+\left[\frac{x}{1-x}+\frac{2(1-x)}{1-\epsilon}\right] \frac{1}{2}\left(\delta^{i j} \delta^{i^{\prime} j^{\prime}}-\delta^{i j^{\prime}} \delta^{i^{\prime} j}\right) \\
&+\frac{x}{1-x} \frac{1}{2}\left(\delta^{i j} \delta^{i^{\prime} j^{\prime}}+\delta^{i j^{\prime}} \delta^{i^{\prime} j}-\frac{1}{1-\epsilon} \delta^{i i^{\prime}} \delta^{j j^{\prime}}\right) \\
&+x(1-x) \frac{k^{i i^{\prime}}}{\boldsymbol{k}^{2}} \delta^{j j^{\prime}}+\frac{1-x}{x} \delta^{i i^{\prime}} \frac{k^{j j^{\prime}}}{\boldsymbol{k}^{2}} \\
&\left.+(1-x)\left(\delta^{i j} \frac{k^{i^{\prime} j^{\prime}}}{\boldsymbol{k}^{2}}+\delta^{i^{\prime} j^{\prime}} \frac{k^{i j}}{\boldsymbol{k}^{2}}-\delta^{i j^{\prime}} \frac{k^{i^{\prime} j}}{\boldsymbol{k}^{2}}-\delta^{i^{\prime} j} \frac{k^{i j^{\prime}}}{\boldsymbol{k}^{2}}\right)\right\}+C_{\mathrm{us}, \epsilon},
\end{aligned}
$$

where we define the symmetric and traceless tensor

$$
v^{i j}=\boldsymbol{v}^{i} \boldsymbol{v}^{j}-\frac{\boldsymbol{v}^{2}}{2(1-\epsilon)} \delta^{i j}
$$

for a given vector $\boldsymbol{v}$ in $2(1-\epsilon)$ transverse dimensions. Using the integral relations in appendix E, we perform the transverse integration and obtain

$$
\begin{aligned}
C_{\mathrm{us}, g g}^{(1) j j^{\prime}, i i^{\prime}}(x, z ;-\infty)=-\frac{\alpha_{s} C_{A}}{\pi}(2 \pi \mu)^{2 \epsilon} \Gamma(1-\epsilon)\left(\frac{\boldsymbol{z}^{2}}{4 \pi}\right)^{\epsilon} \\
\times\left\{\frac{1}{\epsilon}\left[\frac{x}{1-x}+\frac{(1-x)\left(1+x^{2}\right)}{x}\right] \frac{1}{2(1-\epsilon)} \delta^{i i^{\prime}} \delta^{j j^{\prime}}\right. \\
\quad+\frac{1}{\epsilon}\left[\frac{x}{1-x}+\frac{2(1-x)}{1-\epsilon}\right] \frac{1}{2}\left(\delta^{i j} \delta^{i^{\prime} j^{\prime}}-\delta^{i j^{\prime}} \delta^{i^{\prime} j}\right) \\
\quad+\frac{1}{\epsilon} \frac{x}{1-x} \frac{1}{2}\left(\delta^{i j} \delta^{i^{\prime} j^{\prime}}+\delta^{i j^{\prime}} \delta^{i^{\prime} j}-\frac{1}{1-\epsilon} \delta^{i i^{\prime}} \delta^{j j^{\prime}}\right) \\
+x(1-x) \frac{z^{i i^{\prime}}}{\boldsymbol{z}^{2}} \delta^{j j^{\prime}}+\frac{1-x}{x} \delta^{i i^{\prime}} \frac{z^{j j^{\prime}}}{\boldsymbol{z}^{2}} \\
\left.\quad+(1-x)\left(\delta^{i j} \frac{z^{i^{\prime} j^{\prime}}}{\boldsymbol{z}^{2}}+\delta^{i^{\prime} j^{\prime}} \frac{z^{i j}}{\boldsymbol{z}^{2}}-\delta^{i j^{\prime}} \frac{z^{i^{\prime} j}}{\boldsymbol{z}^{2}}-\delta^{i^{\prime} j} \frac{z^{i j^{\prime}}}{\boldsymbol{z}^{2}}\right)\right\}+C_{\mathrm{us}, \epsilon .}
\end{aligned}
$$

In (7.33) and (7.35) we have organised the tensor structure into terms that are diagonal or antisymmetric or symmetric and traceless in the index pair $j j^{\prime}$, for reasons that will become clear in section 7.3.3. We note that the tensor in parentheses in the last line of (7.35) is nonzero in a generic number $2-2 \epsilon$ of transverse dimensions but vanishes for $\epsilon=0$. This is easily seen by contracting this tensor with itself, which gives an expression proportional to $\epsilon$.

We see that (7.35) has poles in $1 / \epsilon$. They correspond to ultraviolet divergences of the matrix element at $\boldsymbol{z}=\mathbf{0}$ and are removed by the counterterm $C_{\mathrm{us}, \epsilon}$. If one splits the $\boldsymbol{k}$ 
integration in (7.33) into the parts going with $e^{i \boldsymbol{k} z}$ and -1 , then the $1 / \epsilon$ poles arise as infrared divergences of the term with $e^{i \boldsymbol{k} z}$, which is ultraviolet finite. The term with -1 involves scaleless integrals, in which the ultraviolet and infrared divergences add up to zero in dimensional regularisation. In the sum of terms, the infrared divergences cancel and the ultraviolet ones remain. The $\overline{\mathrm{MS}}$ counterterm reads

$$
\begin{aligned}
C_{\mathrm{us}, \epsilon}=\frac{\alpha_{s} C_{A}}{\pi} \frac{S_{\epsilon}}{\epsilon} & \left\{\left[\frac{x}{1-x}+\frac{(1-x)\left(1+x^{2}\right)}{x}\right] \frac{1}{2(1-\epsilon)} \delta^{i i^{\prime}} \delta^{j j^{\prime}}\right. \\
& +\left[\frac{x}{1-x}+2(1-x)\right] \frac{1}{2}\left(\delta^{i j} \delta^{i^{\prime} j^{\prime}}-\delta^{i j^{\prime}} \delta^{i^{\prime} j}\right) \\
& \left.+\frac{x}{1-x} \frac{1}{2}\left(\delta^{i j} \delta^{i^{\prime} j^{\prime}}+\delta^{i j^{\prime}} \delta^{i^{\prime} j}-\frac{1}{1-\epsilon} \delta^{i i^{\prime}} \delta^{j j^{\prime}}\right)\right\},
\end{aligned}
$$

where

$$
S_{\epsilon}=\left(4 \pi e^{-\gamma_{E}}\right)^{\epsilon} .
$$

The origin of the factor $1 /(1-\epsilon)$ multiplying $\delta^{i i^{\prime}} \delta^{j j^{\prime}}$ is explained in section 7.3.3; note that the naive implementation of $\overline{\mathrm{MS}}$ counterterms as "the residue of $1 / \epsilon$ times $S_{\epsilon}$ " would lead to an incorrect result.

We now turn to the matching coefficient of the soft factor, which comes from the graph in figure 11c and its complex conjugate. Taking again the limit $Y_{L} \rightarrow-\infty$, we obtain

$$
\begin{aligned}
& C_{S, g}^{(1)}\left(\boldsymbol{z} ; Y_{C}+\infty\right)=\int \frac{d^{4-2 \epsilon} \ell}{(2 \pi)^{4-2 \epsilon}}\left(e^{-i \ell z}-1\right) 2 \pi \delta\left(\ell^{2}\right) \theta\left(\ell^{+}\right) \frac{\delta_{a a^{\prime}} \delta_{c c^{\prime}}}{N^{2}-1} \\
& \times \frac{i}{\ell v_{C}+i 0}\left(-g \mu^{\epsilon} v_{C}^{\nu^{\prime}} f^{a^{\prime} b^{\prime} c^{\prime}}\right)\left(-g_{\nu \nu^{\prime}} \delta_{b b^{\prime}}\right)\left(g \mu^{\epsilon} n^{\nu} f^{c b a}\right) \frac{i}{\ell n-i 0}+\text { c.c. }+C_{S, \epsilon} \\
& =\frac{\alpha_{s} C_{A}}{4 \pi^{2}}(2 \pi \mu)^{2 \epsilon} \int d^{2-2 \epsilon} \ell\left(e^{-i \ell z}-1\right)\left[\int_{0}^{\infty} \frac{d \ell^{+}}{\ell^{+}} \frac{2 v_{C}^{+}}{2\left(\ell^{+}\right)^{2} v_{C}^{-}+\ell^{2} v_{C}^{+}-i 0}\right. \\
& \left.-\frac{2}{\ell^{2}} \int_{0}^{x p^{+}} \frac{d \ell^{+}}{\ell^{+}}+\frac{2}{\ell^{2}} \int_{0}^{x p^{+}} \frac{d \ell^{+}}{\ell^{+}}\right]+ \text {c.c. }+C_{S, \epsilon} \\
& =-\frac{\alpha_{s} C_{A}}{2 \pi^{2}}(2 \pi \mu)^{2 \epsilon} \int \frac{d^{2-2 \epsilon} \boldsymbol{\ell}}{\ell^{2}}\left(e^{-i \ell \boldsymbol{z}}-1\right)\left[\log \frac{2\left(x p^{+}\right)^{2} e^{-2 Y_{C}}}{\ell^{2}}-2 \int_{0}^{x p^{+}} \frac{d \ell^{+}}{\ell^{+}}\right]+C_{S, \epsilon},
\end{aligned}
$$

where $C_{S, \epsilon}$ is the ultraviolet counterterm and "c.c." denotes the complex conjugate term (which results in an extra factor of 2 in the last line). The eikonal propagator depending on $n$ gives rise to the divergent integral over $1 / \ell^{+}$in the third line. To combine this efficiently with the divergence from $C_{\mathrm{us}, g g}$, we have subtracted and added a divergent integral in the fourth line. The first two terms in square brackets then combine to a finite integral, which gives the logarithm in the last line. Having rewritten $v_{C}^{-} / v_{C}^{+}=-e^{-2 Y_{C}}$, we recognise the variable $\zeta$ from (3.50) appearing in this logarithm.

In the remaining divergent integral, we now change variables as $\ell^{+}=p^{+}\left(1-x^{\prime}\right)$, motivated by the corresponding form of the gluon plus-momentum $p^{+}-k^{+}=p^{+}(1-x)$ 
crossing the cut in figure 11b. Performing the Fourier transform using (E.1) and (E.2), we then obtain

$$
\begin{aligned}
C_{S, g}^{(1)}\left(\boldsymbol{z} ; Y_{C}+\infty\right)= & -\frac{\alpha_{s} C_{A}}{2 \pi}(4 \pi)^{\epsilon}\left(\frac{\mu^{2} \boldsymbol{z}^{2}}{4}\right)^{\epsilon} \frac{\Gamma(1-\epsilon)}{\epsilon} \\
& \times\left[\log \frac{4}{\zeta \boldsymbol{z}^{2}}-\gamma_{E}+\psi(-\epsilon)+2 \int_{1-x}^{1} \frac{d x^{\prime}}{1-x^{\prime}}\right]+C_{S, \epsilon}
\end{aligned}
$$

where $\psi(z)=\frac{d}{d z} \log \Gamma(z)$ is the digamma function. Its expansion around $\epsilon=0$ gives $\psi(-\epsilon)=1 / \epsilon+\ldots$, so that overall we obtain a double pole in $\epsilon$. We recall that for a one-loop quantity that has the form

$$
\frac{R_{-2}}{\epsilon^{2}}+\frac{R_{-1}}{\epsilon}+\mathcal{O}\left(\epsilon^{0}\right)
$$

the $\overline{\mathrm{MS}}$-counterterm is given by

$$
-S_{\epsilon}\left(\frac{R_{-2}}{\epsilon^{2}}+\frac{R_{-1}-S_{1} R_{-2}}{\epsilon}\right)
$$

times the tree-level term $C_{S, g}^{(0)}=1$, where the coefficient $S_{1}$ comes from the Taylor expansion $S_{\epsilon}=\left(4 \pi \exp \left(-\gamma_{E}\right)\right)^{\epsilon}=1+S_{1} \epsilon+\mathcal{O}\left(\epsilon^{2}\right)$. The additional contribution $S_{\epsilon} S_{1} R_{-2} / \epsilon$ compensates the $1 / \epsilon$ term that arises from Taylor expanding $S_{\epsilon} R_{-2} / \epsilon^{2}$. Implementing this prescription, we obtain the final form for the soft matching coefficient:

$$
C_{S, g}^{(1)}\left(\boldsymbol{z} ; Y_{C}+\infty\right)=-\frac{\alpha_{s} C_{A}}{2 \pi}\left[-\frac{1}{2} L^{2}+L \log \frac{\mu^{2}}{\zeta}-\frac{\pi^{2}}{12}+2 L \int_{1-x}^{1} \frac{d x^{\prime}}{1-x^{\prime}}\right]
$$

with

$$
L=\log \frac{\mu^{2} \boldsymbol{z}^{2}}{b_{0}^{2}},
$$

where $b_{0}$ is given in (6.25). We note in passing that $C_{S, q}$ is given by the same expression, with the colour factor $C_{A}$ replaced by $C_{F}$. Defining

$$
S_{L}=-\frac{1}{2} L^{2}+L \log \frac{\mu^{2}}{\zeta}-\frac{\pi^{2}}{12}
$$

and combining $C_{\mathrm{us}, g g}$ with $C_{S, g}$ according to (7.31), we obtain the full matching coefficient in tensor form:

$$
\begin{aligned}
& C_{g g}^{(1) j j^{\prime}, i i^{\prime}}(x, \boldsymbol{z} ; \mu, \zeta) \\
& =\frac{\alpha_{s} C_{A}}{2 \pi}\left\{-2 L\left[\frac{x}{(1-x)_{+}}+\frac{(1-x)\left(1+x^{2}\right)}{x}\right]+S_{L} \delta(1-x)\right\} \frac{1}{2} \delta^{i i^{\prime}} \delta^{j j^{\prime}} \\
& +\frac{\alpha_{s} C_{A}}{2 \pi}\left\{-2 L\left[\frac{x}{(1-x)_{+}}+2(1-x)\right]-4(1-x)+S_{L} \delta(1-x)\right\} \frac{1}{2}\left(\delta^{i j} \delta^{i^{\prime} j^{\prime}}-\delta^{i j^{\prime}} \delta^{i^{\prime} j}\right) \\
& +\frac{\alpha_{s} C_{A}}{2 \pi}\left\{-2 L \frac{x}{(1-x)_{+}}+S_{L} \delta(1-x)\right\} \frac{1}{2}\left(\delta^{i j} \delta^{i^{\prime} j^{\prime}}+\delta^{i j^{\prime}} \delta^{i^{\prime} j}-\delta^{i i^{\prime}} \delta^{j j^{\prime}}\right) \\
& -\frac{\alpha_{s} C_{A}}{2 \pi} 2 x(1-x) \frac{z^{i i^{\prime}}}{\boldsymbol{z}^{2}} \delta^{j j^{\prime}}-\frac{\alpha_{s} C_{A}}{2 \pi} \frac{2(1-x)}{x} \delta^{i i^{\prime}} \frac{z^{j j^{\prime}}}{\boldsymbol{z}^{2}} .
\end{aligned}
$$


The rapidity divergences in the $C_{\mathrm{us}, g g}$ and $C_{S, g}$ have been combined into the plusdistribution

$$
\frac{1}{(1-x)_{+}}=\frac{1}{1-x}-\delta(1-x) \int_{0}^{1} \frac{d x^{\prime}}{1-x^{\prime}},
$$

which gives a finite result in convolution integrals. The lower integration limit of the $x^{\prime}$ integral has changed from $1-x$ in (7.42) to 0 in (7.46) because $C_{S, g}$ is multiplied with $\delta(1-x)$ in the combination formula (7.31).

Note that the method we have used avoids introducing the antisymmetric tensor $\epsilon_{\lambda \mu \nu \rho}$ in $D=4-2 \epsilon$ dimensions. With the final result (7.45) in the $D=4$ physical dimensions, it is straightforward to perform a projection onto unpolarised or longitudinally polarised gluons. On the operator side, one uses the spin projectors $\delta^{j j^{\prime}}$ or $i \epsilon^{j j^{\prime}}$ from (3.7), and for the gluon states in the matrix element, one contracts with $\delta^{i i^{\prime}} / 2$ or $-i \epsilon^{i i^{\prime}} / 2$, which gives the average or half the difference of the two helicity states, respectively. ${ }^{14}$ For linear gluon polarisation, we see that $\tau^{j j^{\prime}, k k^{\prime}}$ in (3.7) projects on the symmetric and traceless part in the indices $j j^{\prime}$, with the result depending on two transverse indices $k k^{\prime}$. Matching coefficients involving linear gluon polarisation thus carry indices, as already remarked earlier.

We will give the results of projecting (7.45) onto definite gluon polarisations after discussing in more detail the renormalisation counterterms we have used in our calculations.

\subsubsection{Subtleties of renormalisation}

As explained earlier, the renormalisation of TMDs involves only virtual graphs, which drop out in the calculation of the one-loop matching coefficients. It thus remains to discuss the renormalisation of the matrix elements $\mathcal{M}_{a b}^{j j^{\prime}, l l^{\prime}}(x)$ and $\mathcal{M}_{S, a}$, which are respectively related with PDFs and with the soft factor at $\boldsymbol{z}=\mathbf{0}$.

Gluon polarisation. We begin with renormalisation of gluon PDFs, which gives the counterterm (7.36) for the real graphs in figure 11. We recall that the renormalisation of a scalar PDF operator (e.g. the one for unpolarised gluons) reads

$$
O(x)=Z\left(x^{\prime}\right) \underset{x}{\otimes} O_{B}\left(x^{\prime}\right)
$$

and gives

$$
\mathcal{M}^{(1)}(x)=Z^{(0)}\left(x^{\prime}\right) \underset{x}{\otimes} \mathcal{M}_{B}^{(1)}\left(x^{\prime}\right)+Z^{(1)}\left(x^{\prime}\right) \underset{x}{\otimes} \mathcal{M}_{B}^{(0)}\left(x^{\prime}\right),
$$

where $Z^{(0)}(x)=\delta(1-x)$ and the first-order term $Z^{(1)}(x)$ is given by the $\overline{\mathrm{MS}}$ prescription. Bare quantities are denoted with a subscript $B$, and we ignore mixing with quark operators for the time being. For the gluon operators $O^{j j^{\prime}}(x)$ with open indices, which we are now interested in, the renormalisation factor $Z$ in (7.47) turns into a tensor with four indices. As explained in section 6.5 of [76], renormalisation is significantly simplified by first splitting the tensor operator into its antisymmetric, traceless symmetric diagonal terms, which in $2-2 \epsilon$ transverse dimensions reads

$$
O^{j j^{\prime}}=\frac{1}{2}\left(O^{j j^{\prime}}-O^{j^{\prime} j}\right)+\frac{1}{2}\left(O^{j j^{\prime}}+O^{j^{\prime} j}+\frac{1}{1-\epsilon} \delta^{j j^{\prime}} O^{k k}\right)-\frac{1}{2(1-\epsilon)} \delta^{j j^{\prime}} O^{k k}
$$

\footnotetext{
${ }^{14}$ The sign difference for longitudinal polarisation reflects that one has polarisation vectors $\varepsilon$ for incoming gluons and $\varepsilon^{*}$ for outgoing ones.
} 
The three terms of this decomposition correspond to different irreducible representations of the rotation group. Therefore, each of them is renormalised by a scalar renormalisation factor, and there is no mixing between them. To implement (7.47) in the tensor case, we project all tensors in $j j^{\prime}$ in the same way, which in particular gives

$$
\delta^{i j} \delta^{i^{\prime} j^{\prime}}=\frac{1}{2}\left(\delta^{i j} \delta^{i^{\prime} j^{\prime}}-\delta^{i j^{\prime}} \delta^{i^{\prime} j}\right)+\frac{1}{2}\left(\delta^{i j} \delta^{i^{\prime} j^{\prime}}+\delta^{i j^{\prime}} \delta^{i^{\prime} j}-\frac{1}{1-\epsilon} \delta^{i i^{\prime}} \delta^{j j^{\prime}}\right)+\frac{1}{2(1-\epsilon)} \delta^{i i^{\prime}} \delta^{j j^{\prime}}
$$

for the tree-level tensors in (7.30). This explains the appearance of the factors $1 /(1-\epsilon)$ in the counterterm (7.36). We note that the coefficients of the diagonal, antisymmetric and traceless symmetric tensors in that term are proportional to the well-known DGLAP kernels for the associated gluon polarisation (apart from the $\delta(1-x)$ terms from virtual graphs), which confirms the consistency of the procedure. Contracting the last term in (7.50) with the projector $\delta^{j j^{\prime}}$ for unpolarised gluons and taking the average over incoming gluon polarisations by contracting with $\delta^{i i^{\prime}} /(2(1-\epsilon))$, one obtains unity, which corresponds to the correct normalisation of the tree-level matrix element.

Notice also that the three terms in (7.50) have the same symmetry properties in the index pairs $j j^{\prime}$ and $i i^{\prime}$, which reflects that there are no transitions between states corresponding to different representations of the rotation group.

Quark polarisation. We now turn our attention to quark distributions and their renormalisation. The graphs to be computed for the matching coefficients are as in figure 11, with appropriate replacements of gluons by quarks and with eikonal lines in the fundamental representation. The spinor indices at the top of the graphs are to be contracted with the Dirac matrix of the relevant operator $O_{q}, O_{\Delta q}$ or $O_{\delta q}$, and corresponding contractions are done for the Dirac indices of the quark states in the matrix element.

As is well known, the treatment of quark polarisation in this context requires a consistent definition of $\gamma_{5}$ and of the $\epsilon$ tensor in dimensional regularisation. We use the scheme of 't Hooft, Veltman and Breitenlohner, Maison (HVBM) [123, 124], where $\gamma_{5}=\frac{i}{4 !} \epsilon_{\lambda \mu \nu \rho} \gamma^{\lambda} \gamma^{\mu} \gamma^{\nu} \gamma^{\rho}$. Here $\epsilon_{\lambda \mu \nu \rho}$ is the usual antisymmetric tensor if all indices are in the four physical dimensions (with $\epsilon_{0123}=+1$ ) and zero otherwise. We also introduce its counterpart $\epsilon^{i j}=\epsilon^{+-i j}$, where $i, j$ take values in the $D-2$ transverse dimensions, as well as the tensor $\bar{\delta}^{i j}$, which equals $\delta^{i j}$ if $i, j=1,2$ and is zero for one or both of $i, j$ in the unphysical dimensions.

For transverse quark polarisation, we avoid the use of $\gamma_{5}$ by taking the Dirac matrix $\Gamma_{\delta q}^{j}$ in the operator $\mathcal{O}_{\delta}$ as

$$
\Gamma_{\delta q}^{j}=\frac{1}{2} \epsilon^{j j^{\prime}} \sigma^{j^{\prime}+}=\frac{i}{2} \epsilon^{j j^{\prime}} \gamma^{j^{\prime}} \gamma^{+}
$$

which is equal to the form $\frac{i}{2} \sigma^{j+} \gamma_{5}$ in (3.4) in the physical dimensions, $j=1,2$, whereas it differs for values of $j$ in the unphysical sector. The fermion trace in the matching coefficient $C_{\delta q \delta q}$ then contains two matrices $\sigma^{j+}$ and $\sigma^{i-}$. This gives exactly the same result as one would obtain for the trace with $\sigma^{j+} \gamma_{5}$ and $\sigma^{i-} \gamma_{5}$ using the "naive dimensional regularisation" prescription, where one assumes that $\gamma_{5}$ has square 1 and anticommutes with all 
$\gamma^{\mu}$. That prescription is often used in the context of transverse quark polarisation, but we prefer the formulation with (7.51). Note that with "naive dimensional regularisation" one cannot treat all polarised matching coefficients on an equal footing, since it can only be used to compute traces with an even number of $\gamma_{5}$ matrices. The matrix $\sigma^{i+}$ has also been used to define quark transversity in the recent work [122], where the matching coefficients for polarised TMDs were revisited in a systematic way.

For longitudinal quark polarisation, the matrix $\gamma_{5}$ is unavoidable. In the HVBM scheme, one can rewrite the Dirac matrix in the operator $\Gamma_{\Delta q}$ as

$$
\Gamma_{\Delta q}=\frac{1}{2} \gamma^{+} \gamma_{5}=-\frac{i}{4} \epsilon^{j j^{\prime}} \gamma^{\left[j^{\prime}\right.} \gamma^{j]} \gamma^{+}
$$

where we define $\gamma^{[i} \gamma^{j]}=\frac{1}{2}\left(\gamma^{i} \gamma^{j}-\gamma^{j} \gamma^{i}\right)$. A corresponding replacement can be made for the matrix $\gamma^{-} \gamma_{5}$ that appears in the fermion trace for matrix elements with incoming longitudinally polarised quarks. Postponing the contraction with the $\epsilon$ tensor to the end of the calculation, one can hence represent longitudinally polarised quarks by an operator $\mathcal{O}_{\Delta q}^{j j^{\prime}}=-\frac{1}{4} \bar{q} \gamma^{\left[j^{\prime}\right.} \gamma^{j]} \gamma^{+} q$ with two transverse indices. This makes it easy to see that one has mixing under renormalisation with longitudinally polarised gluons, represented by the operator $\mathcal{O}_{\Delta g}^{j j^{\prime}}=G^{+\left[j^{\prime}\right.} G^{+j]}$ that has the same transformation behaviour under rotations. For all matching coefficients with longitudinal polarisation, $\Delta q \Delta q, \Delta g \Delta q, \Delta q \Delta g$ and $\Delta g \Delta g$, one then has a tensor matching coefficient $C_{a b}^{j j^{\prime}, i i^{\prime}}$ that is antisymmetric in $j j^{\prime}$. As the only terms with ultraviolet divergences in a matching coefficient arise from the matrix elements at $\boldsymbol{z}=\mathbf{0}$, they must be independent of $\boldsymbol{z}$. This leaves $\delta^{i j} \delta^{i^{\prime} j^{\prime}}-\delta^{i j^{\prime}} \delta^{i^{\prime} j}$ as the only possible tensor structure of the UV divergent part of the matching coefficient. This is also the tensor structure of the tree-level matrix element, which multiplies the one-loop counterterm according to (7.48). For the renormalised matching coefficient one thus has

$$
\begin{aligned}
C^{(1) j j^{\prime}, i i^{\prime}}= & \frac{1}{2}\left(\delta^{i j} \delta^{i^{j^{\prime} j^{\prime}}}-\delta^{i j^{\prime}} \delta^{i^{\prime} j}\right) B^{(1)}(x, \epsilon)-\frac{1}{2}\left(\delta^{i j} \delta^{i^{\prime} j^{\prime}}-\delta^{i j^{\prime}} \delta^{i^{\prime} j}\right) Z^{(1)}(x, \epsilon) \\
& +\{\text { terms finite for } \epsilon \rightarrow 0\}
\end{aligned}
$$

where $B^{(1)}$ contains all ultraviolet divergent parts of the bare matrix element and $Z^{(1)}$ is the relevant one-loop renormalisation factor. The $\overline{\mathrm{MS}}$ prescription fixes the latter to be $S_{\epsilon}$ times the pole part of $B^{(1)}$. The outcome of this discussion is that one obtains the same counterterm (and hence the same renormalised result) when using the tensor form (7.53) or when contracting it with

$$
\frac{1}{2} \epsilon^{j j^{\prime}} \epsilon^{i i^{\prime}}=\frac{1}{2}\left(\bar{\delta}^{i j} \bar{\delta}^{i^{\prime} j^{\prime}}-\bar{\delta}^{i j^{\prime}} \bar{\delta}^{i^{\prime} j}\right)
$$

and working with scalar matching coefficients, which corresponds to using the conventional operators $\frac{1}{2} \bar{q} \gamma^{+} \gamma_{5} q$ and $i \epsilon^{j j^{\prime}} G^{+j^{\prime}} G^{+j}$ in the HVBM scheme. Working with (7.53) has the advantage that one does not need to distinguish between physical and unphysical dimensions during the computation.

In the computations of [85] and [122], the tensor $\bar{\delta}$ on the r.h.s. of (7.54) was replaced by the full transverse metric $\delta$ in $D-2$ dimensions, as a modification of the proposal 
by Larin [125], where $\epsilon^{\lambda \mu \nu \rho} \epsilon^{\lambda^{\prime} \mu^{\prime} \nu^{\prime} \rho^{\prime}}$ was replaced by products of $D$ dimensional instead of 4 dimensional metric tensors (cf. [126] for a recent discussion). We see that one obtains the same result when one contracts (7.53) with $\frac{1}{2}\left(\delta^{i j} \delta^{i^{\prime} j^{\prime}}-\delta^{i j^{\prime}} \delta^{i^{\prime} j}\right)$ instead of (7.54) and imposes $\overline{\mathrm{MS}}$ subtraction. The essential point for this is that $B^{(1)}$ and $Z^{(1)}$ have the same $\epsilon$ dependent prefactor in this case. Since poles in $1 / \epsilon$ cancel in $B^{(1)}-Z^{(1)}$ by construction, only the $\epsilon \rightarrow 0$ limit of their prefactor enters in the renormalised matching coefficient. The preceding argument readily generalises to order $\alpha_{s}^{n}$, where one should replace $B^{(1)}-Z^{(1)}$ with $\sum_{m} B^{(n-m)} \otimes Z^{(m)}$, where $B^{(0)}=Z^{(0)}=\delta(1-x)$. If, however, the divergent part of a quantity involves different tensor structures, then the procedure just described is no longer guaranteed to work correctly.

We note that (7.52) is a special case of the identity

$$
\frac{1}{2}\left(\gamma_{\lambda} \gamma_{5}-\gamma_{5} \gamma_{\lambda}\right)=\frac{i}{3 !} \epsilon_{\lambda \mu \nu \rho} \gamma^{[\mu} \gamma^{\nu} \gamma^{\rho]}
$$

in $D$ dimensions, which has been used for a long time when discussing the axial current in the HVBM scheme [76, 125]. The explicit antisymmetrisation on the l.h.s. of (7.55), necessary to make the current Hermitian, can be omitted in (7.52) because $\left\{\gamma^{+}, \gamma_{5}\right\}=0$. As shown in $[76,125]$, the divergence of the flavour nonsinglet axial current is nonzero if one uses $\overline{\mathrm{MS}}$ renormalisation and the HVBM scheme, but a zero divergence can be achieved by an additional finite renormalisation. The resulting nonsinglet current has zero anomalous dimension. At order $\alpha_{s}$, the same finite renormalisation achieves that the divergence of the flavour singlet axial current is given by the Adler-Bell-Jackiw anomaly, as shown in [125] (at higher orders, the required renormalisation factors differ for the flavour singlet and nonsinglet currents). In [127, 128] this finite renormalisation was extended to the nonlocal axial current $\mathcal{O}_{\Delta q}$ that defines longitudinally polarised quark distributions, see also $[129,130]$. The finite renormalisation can be written as

$$
O_{\Delta q}^{\mathrm{NS}}=Z_{5}^{\mathrm{NS}} \otimes O_{\Delta q, \overline{\mathrm{MS}}}^{\mathrm{NS}}, \quad O_{\Delta q}^{\mathrm{S}}=Z_{5}^{\mathrm{S}} \otimes O_{\Delta q, \overline{\mathrm{MS}}}^{\mathrm{S}}, \quad O_{\Delta g}=O_{\Delta g, \overline{\mathrm{MS}}}
$$

for the flavour singlet and nonsinglet operators in $x$ space, where the currents on the r.h.s. are renormalised by the standard $\overline{\mathrm{MS}}$ prescription. At order $\alpha_{s}$ one has

$$
Z_{5}^{\mathrm{NS}}(x)=Z_{5}^{\mathrm{S}}(x)=1-\frac{\alpha_{s}}{2 \pi} 4 C_{F}(1-x)
$$

higher orders can be found in [131]. ${ }^{15}$ This finite renormalisation achieves in particular that the DGLAP kernels $P_{q q}$ and $P_{\Delta q \Delta q}$ agree up to order $\alpha_{s}^{2}[127,128]$. It also achieves that the hard-scattering coefficients of the Drell-Yan process in collinear factorisation satisfy $W_{\Delta q \Delta \bar{q}}=-W_{q \bar{q}}$ at order $\alpha_{s}[129,132]$, where $W_{\Delta q \Delta \bar{q}}$ is for the incoming quark and antiquark both being longitudinally polarised and $W_{q \bar{q}}$ for both being unpolarised. In the results for the matching kernels given in the next subsection, we have included the finite renormalisation (7.56). At the order we are working at, this only affects the coefficient $C_{\Delta q \Delta q}$.

\footnotetext{
${ }^{15}$ Note that $Z_{5}$, called $Z$ in [131], is different from $Z^{5}$ given in [128]. In that work, the renormalisation of the bare current is written in terms of the product $Z^{-1} Z^{5}$ of two renormalisation factors (or matrices in the singlet sector), where $Z^{-1}$ does not correspond to the $\overline{\mathrm{MS}}$ prescription.
} 


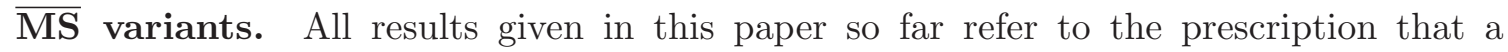
renormalisation factor $Z$ has the form

$$
Z=\sum_{n=0}^{\infty}\left(S_{\epsilon} \alpha_{s}\right)^{n} Z^{(n)},
$$

where $\alpha_{s}$ is the renormalised coupling and $Z^{(n)}$ for $n \geq 1$ is a finite sum of poles in $\epsilon$, fixed uniquely by the requirement that the renormalised quantity be finite at $\epsilon=0$. Equivalently, one may express all quantities in terms of the rescaled coupling $\bar{\alpha}_{s}=S_{\epsilon} \alpha_{s}$ and use minimal subtraction, where all counterterms are sums of poles. The factor $S_{\epsilon}$ is given in (7.37) and was introduced in [133] to simplify the finite terms left after renormalisation.

A variant of the scheme has been proposed in chapter 3.2.6 of [65], where instead of $S_{\epsilon}$ in $(7.37)$ one uses

$$
S_{\epsilon}^{\mathrm{JCC}}=\frac{(4 \pi)^{\epsilon}}{\Gamma(1-\epsilon)}
$$

The difference between the two versions is

$$
S_{\epsilon}-S_{\epsilon}^{\mathrm{JCC}}=\frac{\pi^{2}}{12} \epsilon^{2}+\mathcal{O}\left(\epsilon^{3}\right)
$$

For one-loop quantities with only a single pole in $\epsilon$, this is of no consequence, but it does matter for quantities with a double pole. From (7.41) we see that the counterterm is shifted by $\pi^{2} / 12$ times the coefficient of the double pole if one uses $S_{\epsilon}^{\mathrm{JCC}}$ instead of $S_{\epsilon}$. For the one-loop matching coefficients, this scheme change affects the soft factor and simply removes the term $-\pi^{2} / 12$ in the quantity $S_{L}$ defined in (7.44).

Like any choice of renormalisation prescription, the choice of $S_{\epsilon}$ must drop out in physical quantities such as the overall cross section. At $\mathcal{O}\left(\alpha_{s}\right)$ this choice affects the matching coefficients ${ }^{R} C_{a b}$, the hard-scattering coefficients $C_{H}^{2}$ (with squared logarithms in (7.2) indicating the presence of double poles $1 / \epsilon^{2}$ before renormalisation), as well as DPDFs in colour non-singlet channels and DTMDs in any colour channel (via one-loop renormalisation of the soft factors in their definitions). Collins-Soper and DGLAP kernels, as well as anomalous dimensions, are not affected by the choice of $S_{\epsilon}$ at $\mathcal{O}\left(\alpha_{s}\right)$.

\subsubsection{Matching coefficients for DTMDs on DPDFs}

We now give our final results for the matching coefficients for DTMDs at large $\boldsymbol{y}$ in the colour singlet sector, which are equal to the matching coefficients for single parton TMDs. We have computed them not only with the rapidity regulator used in this paper, but also with the so-called $\delta$ regulator specified in [60], and we find complete agreement between the two versions. 
In the gluon sector, we start from the tensor form (7.45) and project onto unpolarised or longitudinally polarised gluons, which gives

$$
\begin{aligned}
C_{g g}(x, \boldsymbol{z})= & \delta(1-x)-L\left[P_{g g}(x)-\frac{1}{2} \delta(1-x) \gamma_{g}\right]+\frac{\alpha_{s}}{2 \pi} C_{A} S_{L} \delta(1-x), \\
C_{\Delta g \Delta g}(x, \boldsymbol{z})= & \delta(1-x)-L\left[P_{\Delta g \Delta g}(x)-\frac{1}{2} \delta(1-x) \gamma_{g}\right]+\frac{\alpha_{s}}{2 \pi} C_{A} S_{L} \delta(1-x) \\
& -\frac{\alpha_{s}}{2 \pi} 4 C_{A}(1-x),
\end{aligned}
$$

where we recall that

$$
L=\log \frac{\mu^{2} \boldsymbol{z}^{2}}{b_{0}^{2}}, \quad S_{L}=-\frac{1}{2} L^{2}+L \log \frac{\mu^{2}}{\zeta}-\sigma \frac{\pi^{2}}{12}
$$

with $\sigma=1$ if one takes $S_{\epsilon}$ from (7.37) for the $\overline{\mathrm{MS}}$ counterterms, whereas $\sigma=0$ if one takes $S_{\epsilon}^{\mathrm{JCC}}$ from (7.59). The DGLAP splitting kernels $P_{g g}(x)$ and $P_{\Delta g \Delta g}(x)$ include a term proportional to the anomalous dimension $\gamma_{g}=\beta_{0} \alpha_{s} /(2 \pi)$, which comes from virtual graphs (see figure 10). This term is subtracted again in the above matching coefficients, which only receive contributions from real graphs as explained earlier. We list all kernels $P_{a b}$ at the end of this subsection.

Matching coefficients involving linear gluon polarisation carry tensor indices, and we extract from (7.45) the forms

$$
\begin{aligned}
C_{\delta g \delta g}^{j j^{\prime}, i i^{\prime}} & =\tau^{j j^{\prime}, i i^{\prime}} C_{\delta g \delta g}, \\
C_{\delta g g}^{j j^{\prime}} & =\left(\frac{\boldsymbol{z}^{j} \boldsymbol{z}^{j^{\prime}}}{\boldsymbol{z}^{2}}-\frac{1}{2} \delta^{j j^{\prime}}\right) C_{\delta g g}, \\
C_{g \delta g}^{i i^{\prime}} & =\left(\frac{2 \boldsymbol{z}^{i} \boldsymbol{z}^{i^{\prime}}}{\boldsymbol{z}^{2}}-\delta^{i i^{\prime}}\right) C_{g \delta g},
\end{aligned}
$$

where $\tau^{j j^{\prime}, i i^{\prime}}$ is defined in (3.8) and the scalar coefficient functions read

$$
\begin{aligned}
C_{\delta g \delta g}(x, \boldsymbol{z}) & =\delta(1-x)-L\left[P_{\delta g \delta g}(x)-\frac{1}{2} \delta(1-x) \gamma_{g}\right]+\frac{\alpha_{s}}{2 \pi} C_{A} S_{L} \delta(1-x), \\
C_{\delta g g}(x, \boldsymbol{z}) & =-\frac{\alpha_{s}}{2 \pi} 2 C_{A} \frac{1-x}{x} \\
C_{g \delta g}(x, \boldsymbol{z}) & =-\frac{\alpha_{s}}{2 \pi} 2 C_{A} x(1-x) .
\end{aligned}
$$

The prefactor in (7.64) has been chosen such that $C_{\delta g g}$ coincides with the corresponding matching coefficient defined for single gluon TMDs in [85, 122].

In the quark sector, we obtain

$$
\begin{aligned}
C_{q q}(x, \boldsymbol{z})= & \delta(1-x)-L\left[P_{q q}(x)-\frac{1}{2} \delta(1-x) \gamma_{q}\right]+\frac{\alpha_{s}}{2 \pi} C_{F} S_{L} \delta(1-x) \\
& +\frac{\alpha_{s}}{2 \pi} C_{F}(1-x), \\
C_{\Delta q \Delta q}(x, \boldsymbol{z})= & C_{q q}(x, \boldsymbol{z}),
\end{aligned}
$$


with $\gamma_{q}=3 C_{F} \alpha_{s} /(2 \pi)$. For transverse quark polarisation, one has again a tensor valued coefficient

$$
C_{\delta q \delta q}^{j, i}=\delta^{j i} C_{\delta q \delta q}
$$

with

$$
C_{\delta q \delta q}(x, \boldsymbol{z})=\delta(1-x)-L\left[P_{\delta q \delta q}(x)-\frac{1}{2} \delta(1-x) \gamma_{q}\right]+\frac{\alpha_{s}}{2 \pi} C_{F} S_{L} \delta(1-x) .
$$

We note that the tensor structure $\boldsymbol{z}^{j} \boldsymbol{z}^{i}$ would be allowed in (7.71) but does not appear at $\mathcal{O}\left(\alpha_{s}\right)$. For transitions between quarks and gluons, we obtain

$$
\begin{aligned}
C_{q g}(x, \boldsymbol{z}) & =-L P_{q g}(x)+\frac{\alpha_{s}}{2 \pi} 2 T_{F} x(1-x), \\
C_{\Delta q \Delta g}(x, \boldsymbol{z}) & =-L P_{\Delta q \Delta g}(x)+\frac{\alpha_{s}}{2 \pi} 2 T_{F}(1-x)
\end{aligned}
$$

and

$$
\begin{aligned}
C_{g q}(x, \boldsymbol{z}) & =-L P_{g q}(x)+\frac{\alpha_{s}}{2 \pi} C_{F} x, \\
C_{\Delta g \Delta q}(x, \boldsymbol{z}) & =-L P_{\Delta g \Delta q}(x)-\frac{\alpha_{s}}{2 \pi} 2 C_{F}(1-x),
\end{aligned}
$$

where we have re-introduced the normalisation factor $T_{F}=1 / 2$. The analogues of (7.67) and (7.68) for quark-gluon transitions are

$$
\begin{aligned}
& C_{\delta g q}(x, \boldsymbol{z})=-\frac{\alpha_{s}}{2 \pi} 2 C_{F} \frac{1-x}{x}, \\
& C_{q \delta g}(x, \boldsymbol{z})=\frac{\alpha_{s}}{2 \pi} 2 T_{F} x(1-x),
\end{aligned}
$$

where the scalar coefficients are defined as in (7.64) and (7.65) with the subscript $g$ replaced by $q$ for the unpolarised parton.

DPDs associated with linear gluon or transverse quark polarisation are tensor valued and can be decomposed into basis tensors that multiply scalar (or pseudoscalar) distributions. We give these decompositions in appendix $\mathrm{F}$ and list the resulting matching equations between DTMDs and DPDFs in appendix G.

Matching coefficients $C_{a b}$ with exactly one longitudinal polarisation $(\Delta g$ or $\Delta q)$ are zero due to parity invariance. Coefficients with exactly one transverse quark polarisation $\delta q$ vanish because the relevant graphs involve the trace of an odd number of $\gamma$ matrices. Due to charge conjugation invariance, the coefficients $C_{a b}$ remain the same if one replaces all quark indices by antiquark ones. These symmetry relations hold at all orders in $\alpha_{s}$. At one-loop level, there are no transitions between quarks and antiquarks or between quarks of different flavours. These appear starting at $\mathcal{O}\left(\alpha_{s}^{2}\right)$.

Most of the above coefficients have been calculated in the literature before. The coefficients in (7.61), (7.62), (7.67) and (7.75) to (7.77) were calculated in [85], using SCET and the $\delta$ regulator in the version of [60]. We find agreement between our results and those in the arXiv version 5 of [85]. Our results in (7.69) to (7.74) agree with [66] (which contains unpolarised results only) and with [121]. The results in (7.61), (7.64), (7.75) and (7.77) 
agree with [59] and [61]. Apart from $C_{\delta g \delta g}, C_{g \delta g}$ and $C_{q \delta g}$, all matching coefficients were recently calculated in [122], using the $\delta$ regulator briefly described in our appendix B. We agree with the results in the arXiv version 2 of [122].

To the best of our knowledge, $C_{\delta g \delta g}, C_{g \delta g}$ and $C_{q \delta g}$ in (7.66), (7.68) and (7.78) have not been given in the literature before. They are not relevant for single parton TMDs in a nucleon, since the PDF for a linearly polarised gluon vanishes in that case. This is because linear gluon polarisation corresponds to the interference between gluons with helicity +1 and -1 in the amplitude and its conjugate.

Let us finally list the leading-order DGLAP splitting functions, which were first derived in $[134,135]$. They are given by

$$
\begin{aligned}
P_{q q}(x) & =\frac{\alpha_{s}}{2 \pi} C_{F} \frac{1+x^{2}}{(1-x)_{+}}+\frac{1}{2} \delta(1-x) \gamma_{q}, \\
P_{\Delta q \Delta q}(x) & =P_{q q}(x), \\
P_{\delta q \delta q}(x) & =\frac{\alpha_{s}}{2 \pi} C_{F} \frac{2 x}{(1-x)_{+}}+\frac{1}{2} \delta(1-x) \gamma_{q}
\end{aligned}
$$

for quark-quark transitions and by

$$
\begin{aligned}
P_{g g}(x) & =\frac{\alpha_{s}}{2 \pi} 2 C_{A}\left[\frac{x}{(1-x)_{+}}+\frac{(1-x)\left(1+x^{2}\right)}{x}\right]+\frac{1}{2} \delta(1-x) \gamma_{g}, \\
P_{\Delta g \Delta g}(x) & =\frac{\alpha_{s}}{2 \pi} 2 C_{A}\left[\frac{x}{(1-x)_{+}}+2(1-x)\right]+\frac{1}{2} \delta(1-x) \gamma_{g}, \\
P_{\delta g \delta g}(x) & =\frac{\alpha_{s}}{2 \pi} 2 C_{A} \frac{x}{(1-x)_{+}}+\frac{1}{2} \delta(1-x) \gamma_{g}
\end{aligned}
$$

for gluons. The splitting functions mixing quarks and gluons are

$$
\begin{aligned}
P_{q g}(x) & =\frac{\alpha_{s}}{2 \pi} T_{F}\left[x^{2}+(1-x)^{2}\right], & P_{g q}(x) & =\frac{\alpha_{s}}{2 \pi} C_{F} \frac{1+(1-x)^{2}}{x}, \\
P_{\Delta q \Delta g}(x) & =\frac{\alpha_{s}}{2 \pi} T_{F}\left[x^{2}-(1-x)^{2}\right], & P_{\Delta g \Delta q}(x) & =\frac{\alpha_{s}}{2 \pi} C_{F} \frac{1-(1-x)^{2}}{x} .
\end{aligned}
$$

The symmetry properties of the matching coefficients $C_{a b}$ detailed below (7.78) also hold for the splitting functions $P_{a b}$. In addition, $P_{g \delta g}=P_{\delta g g}=0$ because the collinear operators $O_{g}(x)$ and $O_{\delta g}^{j j^{\prime}}(x)$ transform differently under rotations and hence cannot mix under renormalisation.

\subsubsection{Colour non-singlet channels}

It is easy to derive the matching coefficients in colour non-singlet channels from the results we have just presented. All real graphs in figure 11 have the same colour factor, so that the colour projections in (7.22) affect all graphs in the same way. The same holds for the graphs for quark-quark transitions and for transitions between quarks and gluons (in the latter case, the soft factor does not contribute to the one-loop matching coefficients and one only needs the analogues of graphs $11 \mathrm{a}$ and $\mathrm{b}$ ). We can therefore write

$$
{ }^{R} C_{a b}^{(1)}(x, \boldsymbol{z} ; \mu, \zeta)=c_{a b}(R){ }^{1} C_{a b}^{(1)}(x, \boldsymbol{z} ; \mu, \zeta) .
$$


The ratios $c_{a b}(R)$ of colour factors between the representation $R$ and the singlet channel can be determined from the basic ladder graphs, which have the topology of figure 11a. They are readily obtained from the evolution kernels given in section 5.1.3 in [27] and read

$$
\begin{aligned}
c_{q q}(8) & =-\frac{1}{N^{2}-1}, & c_{g q}(A) & =c_{q g}(A)=\sqrt{\frac{N^{2}}{2\left(N^{2}-1\right)}}, \\
c_{g g}(A) & =c_{g g}(S)=\frac{1}{2}, & c_{g q}(S) & =c_{q g}(S)=\sqrt{\frac{N^{2}-4}{2\left(N^{2}-1\right)}}
\end{aligned}
$$

and for $N=3$

$$
c_{g g}(D)=0, \quad c_{g g}(27)=-1 / 3 .
$$

For transitions involving antiquarks one has $c_{\bar{q} \bar{q}}(8)=c_{q q}(8), c_{g \bar{q}}(S)=c_{\bar{q} g}(S)=c_{q g}(S)$ and $c_{g \bar{q}}(A)=c_{\bar{q} g}(A)=-c_{q g}(A)$. The minus sign in the last case reflects the fact that two gluons coupled to an antisymmetric octet have negative charge parity.

We can now derive the explicit form of the LO DGLAP kernels in a general colour representation $R$. For this, we use the evolution equation (6.19) for the matching coefficients ${ }^{R} C_{a b}$. At order $\alpha_{s}$, we can replace ${ }^{R} C_{a b}$ on the r.h.s. by its lowest-order value $\delta_{a b} \delta\left(1-x^{\prime}\right)$ and thus obtain

$$
{ }^{R} P_{a c}(x ; \mu, \zeta)=\frac{1}{2} \delta_{a c} \delta(1-x) \gamma_{F, c}(\mu, \zeta)-\frac{1}{2} \frac{\partial}{\partial \log \mu}{ }^{R} C_{a c}(x, \boldsymbol{z} ; \mu, \zeta) .
$$

Combining this with (7.87), we readily find that the leading-order DGLAP evolution kernels of collinear DPDs in colour non-singlet channels are given by

$$
\begin{aligned}
{ }^{R} P_{a b}(x ; \mu, \zeta)= & \frac{1}{2} \delta_{a b} \delta(1-x) \gamma_{F, a}(\mu, \zeta)-\frac{c_{a b}(R)}{2} \frac{\partial}{\partial \log \mu}{ }^{1} C_{a b}(x, \boldsymbol{z} ; \mu, \zeta) \\
= & \frac{1-c_{a a}(R)}{2} \delta_{a b} \delta(1-x) \gamma_{F, a}(\mu, \zeta)+c_{a b}(R){ }^{1} P_{a b}(x ; \mu) \\
= & -\frac{1}{2} \delta_{a b} \delta(1-x){ }^{R} \gamma_{J}(\mu) \log \frac{\sqrt{\zeta}}{\mu} \\
& +\frac{1-c_{a a}(R)}{2} \delta_{a b} \delta(1-x) \gamma_{a}(\mu)+c_{a b}(R){ }^{1} P_{a b}(x ; \mu) .
\end{aligned}
$$

They are hence easily recovered from their counterparts in the colour singlet channel. In the last step we have brought the kernel into the form (5.26), using the explicit $\zeta$ dependence (2.5) of $\gamma_{F, a}$, the general relation (6.17) between anomalous dimensions, and the one-loop relation ${ }^{R} \gamma_{K, a}=c_{a a}(R)^{1} \gamma_{K, a}$, which follows from (7.87). We recall that the colour singlet kernels ${ }^{1} P_{a a}(x ; \mu)$ contain a contribution $\delta(1-x) \gamma_{a} / 2$ from virtual graphs, see (7.79) to (7.84). The last line of (7.91) simply reflects that these graphs have the same colour factor for all channels $R$.

Using (7.91), one can rewrite the $\zeta$ independent distributions $\widehat{F}$ introduced in (5.27) as

$$
\begin{aligned}
& { }^{R} \widehat{F}_{a_{1} a_{2}, \mu_{0}, \zeta_{0}}\left(x_{i}, \boldsymbol{y} ; \mu_{i}\right)=\exp \left[\left(1-c_{a_{1} a_{1}}(R)\right) \int_{\mu_{0}}^{\mu_{1}} \frac{d \mu}{\mu} \gamma_{a_{1}}(\mu)\right. \\
& \left.+\left(1-c_{a_{2} a_{2}}(R)\right) \int_{\mu_{0}}^{\mu_{2}} \frac{d \mu}{\mu} \gamma_{a_{2}}(\mu)\right]{ }^{R} \widetilde{F}_{a_{1} a_{2}, \mu_{0}, \zeta_{0}}\left(x_{i}, \boldsymbol{y} ; \mu_{i}\right),
\end{aligned}
$$


where $\widetilde{F}$ satisfies the evolution equation (5.28) with kernels $c_{a_{1} b_{1}}(R){ }^{1} P_{a_{1} b_{1}}\left(x_{1}^{\prime} ; \mu_{1}, \mu_{1}^{2}\right)$ instead of ${ }^{R} P_{a_{1} b_{1}}\left(x_{1}^{\prime} ; \mu_{1}, \mu_{1}^{2}\right)$. One can thus use numerical code for the one-loop evolution of colour singlet DPDs by rescaling the evolution kernels.

\subsection{Splitting kernels for DPDs}

Both DTMDs and DPDFs can be matched on single-parton distributions at small $\boldsymbol{y}$ as specified in (5.37), (6.38) and (6.42). At $\mathcal{O}\left(\alpha_{s}\right)$, all three matching equations involve the same kernels ${ }^{R} T_{a_{0} \rightarrow a_{1} a_{2}}^{l l^{\prime}}$, which were computed in section 5.2.2 of [27]. We list them here in the notation of [30] (arXiv version 2), where compared to [27] the kernels $T$ have the opposite order of indices and include a colour factor. Thus one has for instance

$$
\left.{ }^{1} T_{g \rightarrow q \bar{q}}^{l l^{\prime}}\right|_{\text {here }}=\left.T_{F} T_{g \rightarrow q \bar{q}}^{l^{\prime} l}\right|_{\text {ref. [27] }},\left.\quad{ }^{1} T_{q \rightarrow g \Delta q}^{l l^{\prime}}\right|_{\text {here }}=\left.C_{F} T_{q \rightarrow g \Delta q}^{l^{\prime} l}\right|_{\text {ref. [27] }} .
$$

The new assignment of indices is such that $l$ refers to the amplitude and $l^{\prime}$ to its complex conjugate. We must note a mistake in equation (5.62) of [27], where the correct order of indices in the kernels $T$ and $U$ is $l^{\prime} l$ and not $l l^{\prime}$.

We only give the kernels for the splitting of an unpolarised parton $a_{0}$ here. In the matching equation (5.37) for DTMDs on TMDs, one can also have transverse quark or linear gluon polarisation for $a_{0}$, but this possibility is absent if one matches on collinear PDFs in an unpolarised proton.

We start with the kernels for DPDs in the colour singlet channel. For a gluon splitting into a quark and an antiquark, one has

$$
\begin{aligned}
{ }^{1} T_{g \rightarrow q \bar{q}}^{l l^{\prime}}(u)= & -{ }^{1} T_{g \rightarrow \Delta q \Delta \bar{q}}^{l l^{\prime}}(u)=T_{F}\left(u^{2}+\bar{u}^{2}\right) \delta^{l l^{\prime}}, \\
{ }^{1} T_{g \rightarrow \Delta q \bar{q}}^{l l^{\prime}}(u)= & -{ }^{1} T_{g \rightarrow q \Delta \bar{q}}^{l l^{\prime}}(u)=-i T_{F}(u-\bar{u}) \epsilon^{l l^{\prime}}, \\
& {\left[{ }^{1} T_{g \rightarrow \delta q \delta \bar{q}}^{l l^{\prime}}(u)\right]^{j j^{\prime}}=-2 T_{F} u \bar{u} \delta^{l l^{\prime}} \delta^{j j^{\prime}}, }
\end{aligned}
$$

where $\bar{u}=1-u$. For a quark splitting into a gluon and a quark, the kernels are given by

$$
\begin{aligned}
{ }^{1} T_{q \rightarrow g q}^{l l^{\prime}}(u) & =C_{F} \frac{1+\bar{u}^{2}}{u} \delta^{l l^{\prime}}, & & { }^{1} T_{q \rightarrow \Delta g q}^{l l^{\prime}}(u)=i C_{F} \frac{1+\bar{u}^{2}}{u} \epsilon^{l l^{\prime}}, \\
{ }^{1} T_{q \rightarrow \Delta g \Delta q}^{l l^{\prime}}(u) & =C_{F}(1+\bar{u}) \delta^{l l^{\prime}}, & & { }^{1} T_{q \rightarrow g \Delta q}^{l l^{\prime}}(u)=i C_{F}(1+\bar{u}) \epsilon^{l l^{\prime}}, \\
{\left[{ }^{1} T_{q \rightarrow \delta g q}^{l l^{\prime}}(u)\right]^{j j^{\prime}} } & =2 C_{F} \frac{\bar{u}}{u} \tau^{l l^{\prime}, j j^{\prime}} & &
\end{aligned}
$$

with $\tau^{l l^{\prime}, j j^{\prime}}$ defined in (3.8). The kernels for an antiquark splitting into a gluon and antiquark have the same form due to charge conjugation invariance. Finally, for a gluon splitting into two gluons one has

$$
\begin{aligned}
{ }^{1} T_{g \rightarrow g g}^{l l^{\prime}}(u) & =2 C_{A}\left(\frac{u}{\bar{u}}+\frac{\bar{u}}{u}+u \bar{u}\right) \delta^{l l^{\prime}}, & & \\
{ }^{1} T_{g \rightarrow \Delta g \Delta g}^{l l^{\prime}}(u) & =2 C_{A}(2-u \bar{u}) \delta^{l l^{\prime}}, & { }^{1} T_{g \rightarrow g \Delta g}^{l l^{\prime}}(u) & =2 i C_{A}\left(2 \bar{u}+\frac{u}{\bar{u}}\right) \epsilon^{l l^{\prime}}, \\
{\left[{ }^{1} T_{g \rightarrow \delta g \delta g}^{l l^{\prime}}(u)\right]^{j j^{\prime}, k k^{\prime}} } & =C_{A} u \bar{u} \delta^{l l^{\prime}} \tau^{j j^{\prime}, k k^{\prime}}, & & {\left[{ }^{1} T_{g \rightarrow g \delta g}^{l l^{\prime}}(u)\right]^{k k^{\prime}}=2 C_{A} \frac{u}{\bar{u}} \tau^{l l^{\prime}, k k^{\prime}} . }
\end{aligned}
$$


All other kernels that cannot be obtained from those above by interchanging $a_{1}$ and $a_{2}$ or by changing quarks into antiquarks in (7.95) are zero. In appendix $\mathrm{H}$ we give the matching equations that follow from these results for the scalar and pseudoscalar DTMDs or DPDFs defined in appendix F.

Each of the above kernels corresponds to exactly one Feynman graph, so that the results for other colour channels can be obtained by changing the overall factor as

$$
{ }^{R} T_{a_{0} \rightarrow a_{1} a_{2}}=c_{a_{0} \rightarrow a_{1} a_{2}}(R){ }^{1} T_{a_{0} \rightarrow a_{1} a_{2}}
$$

with

$$
\begin{aligned}
& c_{q \rightarrow g q}(A)=-\frac{N}{\sqrt{2}}, \\
& c_{q \rightarrow g q}(S)=\sqrt{\frac{N^{2}-4}{2}} \\
& c_{g \rightarrow g g}(A)=-\frac{\sqrt{N^{2}-1}}{2}, \quad c_{g \rightarrow g g}(S)=-c_{g \rightarrow g g}(A), \\
& c_{g \rightarrow g g}(D)=0, \quad c_{g \rightarrow g g}(27)=-\sqrt{3} . \\
& c_{g \rightarrow q \bar{q}}(8)=-\frac{1}{\sqrt{N^{2}-1}}
\end{aligned}
$$

For the colour representations $D$ and 27, we have given the results for $N=3$. Notice that in all cases except $c_{g \rightarrow q \bar{q}}(8)$ and $c_{g \rightarrow g g}(D)$, the colour non-singlet channel is enhanced over the singlet one for $\mathrm{SU}(3)$.

\section{Summary}

We have performed a systematic analysis of double parton scattering for measured transverse momenta, extending the framework for factorisation and resummation formulated in [65] for single parton scattering with colourless particles in the final state. A major challenge is the description of soft gluon exchange and the associated rapidity evolution, which has a much richer colour structure in DPS than in SPS. We handle this structure by projecting suitable pairs of partons onto definite colour representations.

Based on perturbative results up to two loops [74], we have proposed equation (3.26) for the rapidity dependence of the soft factor in DPS, which is a Collins-Soper equation for matrices in colour space. Assuming the validity of that equation, we have given a concise definition of DPDs in (3.29) and derived their general properties. Applied to SPS, our construction provides an alternative form for the square root factor in the definition of single-parton TMDs by Collins [65]. Transverse momentum dependent DPDs (DTMDs) follow the evolution equations (5.7) and (5.11), which can be solved in closed analytic form as given in (5.17). The rapidity evolution of DTMDs mixes different colour channels, and its solution involves a matrix exponential in the space of colour representations. One-loop expressions of the evolution kernels and anomalous dimensions are given in section 7.2 for all parton combinations and colour channels.

In collinear factorisation, i.e. when the transverse momenta of the final-state particles are integrated over, the soft factor for DPS simplifies considerably: it becomes diagonal in colour representation space and independent of the parton type. This can be shown 
using only colour algebra and the fact that pairs of Wilson lines associated with initial and final state partons cancel as $W W^{\dagger}=\mathbb{1}$. Important consequences of this result are the simple structure (4.53) of the cross section for DPS processes producing coloured particles, as well as the fact that evolution in rapidity does not mix different colour channels for collinear DPDs (called DPDFs here). The corresponding evolution equations can be found in (5.23) and (5.24), and an analytic expression that makes the rapidity dependence explicit is provided in (5.27). At one-loop accuracy, the evolution kernels in colour non-singlet channels are related in a simple way to the usual DGLAP kernels, as specified in (7.91).

If the size $q_{T}$ of the measured transverse momenta in a DPS process is large compared with nonperturbative scales $\Lambda$ (but still small compared with the scale $Q$ of the hardscattering processes), one can use TMD factorisation with DTMDs expressed in terms of perturbative kernels and simpler hadronic matrix elements. There are two regimes for this short-distance matching, depending on the relative size of the transverse distance $\boldsymbol{y}$ between the two partons and the distances $\boldsymbol{z}_{1}$ and $\boldsymbol{z}_{2}$ that are conjugate to the measured transverse momenta in the cross section formula.

In the first regime, when $\boldsymbol{y}$ is much larger than $\boldsymbol{z}_{1}$ and $\boldsymbol{z}_{2}$, the matching is very similar to the familiar matching of single parton TMDs onto single parton PDFs. The resulting expressions are given in (6.22) and (6.24). Apart from a suppression factor for colour non-singlet DPDFs, which is controlled by their rapidity evolution kernel ${ }^{R} J(\boldsymbol{y})$, we obtain a product of Sudakov exponentials and of matching kernels (one for each parton) with DPDFs. At one-loop order, the matching kernels ${ }^{R} C(x, \boldsymbol{z})$ are due to ladder-type graphs and coincide with the ones for single-parton TMDs in the colour singlet channel $R=1$. Most of these kernels have been given in the literature before, but we have recomputed them in section 7.3 and extended the calculation to provide results for all combinations of parton type, polarisation and colour needed for DPS. We use the HVBM scheme for defining $\gamma_{5}$ and the $\epsilon$ tensor in dimensional regularisation, but point out a way to avoid these quantities before taking the number of dimensions to $D=4$.

In the second regime, when $\boldsymbol{y}$ is of the same size as $\boldsymbol{z}_{1}$ and $\boldsymbol{z}_{2}$, one can match the DTMDs onto collinear distributions of twist two (i.e. usual PDFs), twist three or twist four. We neglect collinear twist-three distributions since in an unpolarised proton they are chiral-odd and hence expected to play a minor role in the regime of small parton momentum fractions typical of DPS processes. The matching onto twist-four distributions is presently only known at lowest order, where it is trivial and given in (6.39). The contribution that matches onto ordinary PDFs corresponds to the splitting of one parton into two. At leading order it has the form (6.38) with splitting kernels given in section 7.4. An important point is that in the DPS cross section, terms matching onto collinear distributions of different twist come with different powers of $\Lambda / q_{T}$ and of $\alpha_{s}$ as specified in (6.40).

To combine the expressions for matching at large or small $\boldsymbol{y}$, we adapt the subtraction formalism of [65]. In this formalism, double counting is avoided by a subtraction term in the cross section. This term can be easily obtained from the small-y or the large- $\boldsymbol{y}$ expression without further computation, but it requires some care when choosing scales as discussed in section 6.4. The same subtraction formalism is used to combine the cross sections for DPS, SPS and their interference as specified in sections 5.3 and 6.5. In the regime where 
$\Lambda \ll q_{T} \ll Q$ one needs a limited set of nonperturbative quantities for computing the cross section: the DPDFs ${ }^{R} F\left(x_{i}, \boldsymbol{y}\right)$ and their rapidity evolution kernel ${ }^{R} J(\boldsymbol{y})$, collinear twist-four distributions (which arise from DPDs in the short-distance limit) and ordinary single-parton PDFs. The colour octet kernel ${ }^{8} J(\boldsymbol{y})$ turns out to be equal to the CollinsSoper kernel for single-gluon TMDs, providing a surprising connection between two rather different areas of factorisation.

In the multi-scale regime $\Lambda \ll q_{T} \ll Q$ we thus have a significantly increased predictive power of the theory. Rather than a huge set of transverse-momentum dependent distributions, the TMD cross section involves the same nonperturbative functions as the cross section integrated over $q_{T}$. All other ingredients in the factorisation formula are of perturbative nature. In most (although not all) cases they are known at least to the first nontrivial order in $\alpha_{s}$. The results presented in this work provide a starting point for phenomenological investigations, for instance of electroweak diboson production with measured transverse boson momenta. An important task will be to assess which types of correlations and which regions of $\boldsymbol{y}$ are important in a given process and kinematical setting.

Before concluding, we wish to discuss the question of scheme dependence in the treatment of large gluon rapidities. Matrix elements of Wilson lines along lightlike directions typically have rapidity divergences, which originate from regions of gluon momenta with infinite rapidity. In the present paper, we follow the approach of Collins [65] and use Wilson line directions away from the light cone to regulate these divergences. The regulated Wilson lines then depend on rapidity variables. In the DPD construction laid out in section 3.3, the parameter $\zeta$ is defined in terms of a central rapidity $Y_{C}$ that specifies the range of gluon rapidities effectively included in the DPD. Several other schemes for regulating rapidity divergences have been proposed and used in the literature, see [60, 78, 99, 136-138] and [139]. Quantities that depend on a regulator variable will in general differ between schemes. By contrast, quantities that enter a cross section formula in the same way in two schemes must be equal. This holds in particular for TMDs, and hence also for the associated Collins-Soper kernels and matching coefficients onto PDFs. Discussions of equivalence between specific schemes can be found in [68, 79, 140].

It follows that our results in sections 4 to 7 are independent of the rapidity regulator scheme. ${ }^{16}$ Of course, this only holds if one defines the variables $\zeta$ and $\bar{\zeta}$ in a consistent way across different schemes, see for instance appendix B here and section 6.1 in [75]. We have checked by explicit calculation that the one-loop matching coefficients presented in section 7.3 remain the same when one uses the $\delta$ regulator specified in [60]. By contrast, our arguments in section 3 are specifically formulated for Wilson lines with finite rapidities and would need to be adapted to other schemes, using an appropriate translation between the regulator variables as given in appendix B for a particular case.

As already noted, we absorb the soft factor into the DPDs, corresponding to what is done for SPS in several TMD factorisation approaches [60,65]. A different route was taken in [28], where the soft factor explicitly appears in the final DPS cross section formula. Such

\footnotetext{
${ }^{16} \mathrm{~A}$ few equations in these sections are obviously specific to the regulator we use, such as the definition (5.8) of $\zeta$ in terms of $Y_{C}$.
} 
an approach is often taken in the SCET literature in situations when the soft factor can be computed in perturbation theory (e.g. for single Drell-Yan production at large $q_{T}$ ). In DPS, however, one needs the soft factor at large distances $\boldsymbol{y}$. Including it into the DPDs therefore limits the number of nonperturbative functions required to compute the cross section.

Given the results of the present paper and the arguments regarding Glauber gluon exchange in [29], we consider that TMD factorisation for DPS is now established at the same level of rigour as for single hard scattering, apart from the following issues. (i): as already mentioned, there is no complete all-order proof of the properties we assumed for the soft factor in section 3.2, but significant process in this direction has been made in [75]. (ii): there is no all-order proof of the nonabelian Ward identities required for factorising soft gluon exchange between left and right moving partons into the soft matrix. (iii): as noted below (7.31), we have omitted Wilson line self interactions in our calculation, following the logic discussed for SPS in chapter 13.7 of [65]. A more thorough understanding of this issue, in particular for DPS, has not yet been achieved. Progress on any of these three points will constitute a further step towards a rigorous treatment of double parton scattering in QCD.

\section{Acknowledgments}

We gratefully acknowledge discussions with Alessandro Bacchetta, John Collins, Tom van Daal, Miguel G. Echevarría, Jonathan Gaunt, Zhongbo Kang, Piet Mulders, Riccardo Nagar, Alexei Prokudin, Ted Rogers, Maximilian Stahlhofen, Iain Stewart, Frank Tackmann, Alexey Vladimirov, Werner Vogelsang and Wouter Waalewijn. TK is supported by the European Community under the "Ideas" program QWORK (contract 320389). He acknowledges the hospitality of the Munich Institute for Astro- and Particle Physics (MIAPP) of the DFG cluster of excellence "Origin and Structure of the Universe".

All figures were made using JaxoDraw [141, 142]. For calculations we have used the FeynCalc package [143, 144], the ColorMath package [145] and FORM [146, 147].

\section{A Matrix manipulations for the soft factor}

Referring to section 3.2, we now show that the set of properties $1 \mathrm{a}-1 \mathrm{c}$ for the soft factor is equivalent with properties $2 \mathrm{a}-2 \mathrm{~b}$. As a corollary, we find that the soft matrix $S(Y)$ is positive definite for arbitrary large $Y$ if these properties hold.

Properties $\mathbf{2 a}-\mathbf{2} \mathbf{b}$ follow from properties $\mathbf{1 a}-\mathbf{1 c}$. The matrix $s(0)$ is nonsingular by assumption (property 1 b), so that one can define a matrix $\widehat{K}$ by

$$
\widehat{K} s(0)=s(0) K .
$$

The differential equation (3.24) (property 1a) is thus solved by

$$
s(Y)=s(0) e^{Y K}=e^{Y \widehat{K}} s(0)
$$

for all $Y$. We now define

$$
\widehat{S}(Y)=s(Y / 2) s^{\dagger}(Y / 2) .
$$

Using (3.25) (property 1c) for $Y_{0}=Y / 2$, we see that $\widehat{S}(Y)$ approximates $S(Y)$ for $Y \gg 1$. 
For any complex vector $a$ one has

$$
a^{\dagger} \widehat{S}(Y) a=b^{\dagger} b
$$

with $b=s^{\dagger}(Y / 2) a$. The expression in (A.4) is obviously positive or zero, and since $s^{\dagger}(Y / 2)$ is nonsingular by assumption (property 1b), it is zero only for $a=0$. Therefore $\widehat{S}(Y)$ is positive definite for all $Y$. This implies property $2 \mathrm{~b}$, and it also implies that $S(Y)$ is positive for all $Y$ where it can be approximated by $\widehat{S}(Y)$.

Multiplying (A.1) with $s^{\dagger}(0)$ on the right, and using that $K$ is Hermitian, we obtain

$$
\widehat{K} \widehat{S}(0)=\widehat{S}(0) \widehat{K}^{\dagger} .
$$

We thus finally have

$$
\widehat{S}(Y)=e^{Y \widehat{K} / 2} \widehat{S}(0) e^{Y \widehat{K}^{\dagger} / 2}=e^{Y \widehat{K}} \widehat{S}(0),
$$

where we used (A.2) and (A.3) in the first step and (A.5) in the second one. This implies property 2 a for $Y \gg 1$, where $\widehat{S}(Y)$ approximates $S(Y)$.

Properties $\mathbf{1 a}-\mathbf{1 c}$ follow from properties $\mathbf{2 a}-\mathbf{2} \mathbf{b}$. We start by defining the matrix $\widehat{S}(Y)$ for all $Y$ by

$$
\widehat{S}(Y)=\lim _{Y_{0} \rightarrow \infty} e^{\left(Y-Y_{0}\right) \widehat{K}} S\left(Y_{0}\right)
$$

This obviously satisfies

$$
\frac{\partial}{\partial Y} \widehat{S}(Y)=\widehat{K} \widehat{S}(Y)
$$

exactly. For $Y, Y_{0} \gg 1$ one has $S(Y)=e^{\left(Y-Y_{0}\right) \widehat{K}} S\left(Y_{0}\right)$ by virtue of (3.26) (property 2a), which implies $\widehat{S}(Y)=S(Y)$ for $Y \gg 1$.

Because $S(Y)$ is Hermitian according to (3.20), it follows from (3.26) that $\widehat{K} S(Y)=$ $S(Y) \widehat{K}^{\dagger}$ for $Y \gg 1$. Using this, one deduces from (A.7) that $\widehat{S}(Y)$ is Hermitian as well. With (A.8) we then have

$$
\widehat{K} \widehat{S}(Y)=\widehat{S}(Y) \widehat{K}^{\dagger}
$$

for all $Y$. The solution of (A.8) can thus be written as

$$
\widehat{S}(Y)=e^{\left(Y-Y_{1}\right) \widehat{K}} \widehat{S}\left(Y_{1}\right)=e^{\left(Y-Y_{1}-Y_{0}\right) \widehat{K}} \widehat{S}\left(Y_{1}\right) e^{Y_{0} \widehat{K}^{\dagger}}
$$

for arbitrary $Y_{0}$ and $Y_{1}$.

We now recall the definition of the square root $M^{1 / 2}$ of a positive definite matrix $M$. One can write $M=V^{\dagger} D V$, where $V$ is unitary and $D$ is diagonal with positive entries $d_{i i}$. One defines $D^{1 / 2}$ as the diagonal matrix with entries $\sqrt{d_{i i}}$, and furthermore $\sqrt{M}=V^{\dagger} D^{1 / 2} V$. If $M$ is real then $V$ can be taken as an orthogonal matrix, so that $M^{1 / 2}$ is real as well. Since $\widehat{S}\left(Y_{1}\right)$ is positive definite for some $Y_{1}$ (property $2 \mathrm{~b}$ ), one can use (A.10) with $Y_{0}=\left(Y-Y_{1}\right) / 2$ and write $\widehat{S}(Y)=t(Y) t^{\dagger}(Y)$, where $t(Y)=e^{\left(Y-Y_{1}\right) \widehat{K} / 2}\left[\widehat{S}\left(Y_{1}\right)\right]^{1 / 2}$ is a nonsingular matrix. With the same argument as given below (A.3), it then follows that $\widehat{S}(Y)$ is positive for all $Y$.

We can now define

$$
s(Y)=e^{Y \widehat{K}}[\widehat{S}(0)]^{1 / 2} U,
$$


where $U$ is a unitary matrix. $s(Y)$ is nonsingular (property $1 \mathrm{~b}$ ) and satisfies the decomposition (3.25) (property 1c) by virtue of (A.10) with $Y_{1}=0$. Defining

$$
K=U^{\dagger}[\widehat{S}(0)]^{-1 / 2} \widehat{K}[\widehat{S}(0)]^{1 / 2} U,
$$

one readily finds that $K^{\dagger}=K$ and

$$
s(Y)=[\widehat{S}(0)]^{1 / 2} U e^{Y K},
$$

which implies (3.24) (property 1a). The definitions (A.11) and (A.12) are consistent with (A.1) above. We note that a more general definition of $s(Y)$ and $K$ is obtained by replacing $\widehat{S}(0) \rightarrow \widehat{S}\left(Y_{1}\right)$ in (A.11) to (A.13), as well as $Y \rightarrow\left(Y-Y_{1} / 2\right)$ in the exponentials there.

Restrictions on $\boldsymbol{U}$. In principle, the matrix $U$ can be chosen freely as a smooth function of $\boldsymbol{z}_{1}, \boldsymbol{z}_{2}$ and $\boldsymbol{y}$, but independent of $Y$ and of the renormalisation scale $\mu$. We restrict ourselves to choices such that $U=1$ for $\boldsymbol{y}=\mathbf{0}$. Since $S(Y)$ is diagonal in that case (see section 4.4), the same then holds for $\widehat{S}(Y), s(Y)$ and for $K=\widehat{K}$.

We furthermore impose a number of restrictions on $U$ in different partonic channels. We thus demand that

$$
U_{q q}=U_{\bar{q} \bar{q}}^{*}, \quad U_{q \bar{q}}=U_{\bar{q} q}^{*}, \quad U_{q g}=U_{\bar{q} g}^{*}, \quad U_{g g}=U_{g g}^{*},
$$

which is necessary to obtain the corresponding relations (3.30) for $s_{a_{1} a_{2}}$ from those for $S_{a_{1} a_{2}}$. As discussed in section 4.3, we also require that $R R^{\prime} U$, defined in analogy to (4.20), is a real valued matrix in representation space and satisfies

$$
\begin{aligned}
{ }^{R R^{\prime}} U_{q q}\left(\boldsymbol{z}_{1}, \boldsymbol{z}_{2}, \boldsymbol{y}\right) & ={ }^{R R^{\prime}} U_{q \bar{q}}\left(\boldsymbol{z}_{1},-\boldsymbol{z}_{2}, \boldsymbol{y}\right) \\
& ={ }^{R R^{\prime}} U_{\bar{q} q}\left(-\boldsymbol{z}_{1}, \boldsymbol{z}_{2}, \boldsymbol{y}\right)={ }^{R R^{\prime}} U_{\bar{q} \bar{q}}\left(-\boldsymbol{z}_{1},-\boldsymbol{z}_{2}, \boldsymbol{y}\right) .
\end{aligned}
$$

We note that the trivial choice $U_{a_{1} a_{2}}=\mathbb{1}$ satisfies all requirements just listed and gives $R R^{\prime} U_{a_{1} a_{2}}=\delta_{R R^{\prime}}$.

\section{B Comparison with the $\delta$ regulator scheme}

As emphasised in section 8, our treatment of soft and collinear factors uses Wilson line directions away from the light cone to regulate rapidity divergences, as was done by Collins in [65]. The regulated Wilson lines then depend on large but finite rapidities.

The two-loop calculation of the DPS soft factor in [74] was performed using the $\delta$ regulator scheme specified in [78, 99]. Given the particular relevance of this calculation for our assumptions on the soft factor in section 3.2, we now compare the variables in that scheme with the ones in the scheme of Collins. For simplicity we perform the comparison for the SPS soft factor. It is easy to extend the following arguments to DPS, including the appropriate rescaling of $\zeta$, see (3.50) and (5.8). We note that there are two earlier variants of the $\delta$ regulator, described in [60] and [140], and compared with the Collins scheme in [79] and [140], respectively. 
In the $\delta$ regulator scheme of $[78,99]$, Wilson lines are taken along lightlike paths but modified by an exponential damping at large distances $z^{-}\left(z^{+}\right)$. This damping is controlled by a parameter $\delta^{+}\left(\delta^{-}\right)$, which transforms like the plus (minus) component of a vector under longitudinal boosts. One has a correspondence of variables

$$
Y_{R} \leftrightarrow \log \frac{\mu}{\delta^{-}}, \quad Y_{L} \leftrightarrow \log \frac{\delta^{+}}{\mu} .
$$

Due to boost invariance, the soft factor $S$ only depends on $Y_{R}-Y_{L} \leftrightarrow-\log \left(\delta^{+} \delta^{-} / \mu^{2}\right)$. The correspondence indicated by $\leftrightarrow$ is valid when taking derivatives, so that $\mu$ in (B.1) could be replaced by another quantity that has dimension of mass and is boost invariant. In our formalism, the soft factor can be split into

$$
S\left(Y_{R}-Y_{L}\right)=\sqrt{S\left(2 Y_{R}-2 Y_{C}\right)} \sqrt{S\left(2 Y_{C}-2 Y_{L}\right)},
$$

and the same splitting is performed with the $\delta$ regulator, with the following correspondence of variables:

$$
2\left(Y_{R}-Y_{C}\right) \leftrightarrow \log \frac{\mu^{2}}{\left(\delta^{-}\right)^{2}} \frac{2\left(\bar{k}^{-}\right)^{2}}{\bar{\zeta}}, \quad 2\left(Y_{C}-Y_{L}\right) \leftrightarrow \log \frac{\mu^{2}}{\left(\delta^{+}\right)^{2}} \frac{2\left(k^{+}\right)^{2}}{\zeta}
$$

Here the momentum components $k^{+}$and $\bar{k}^{-}$correspond to the two partons initiating a hard scattering, and we have normalised the rapidity parameters as $\zeta \bar{\zeta}=\left(2 k^{+} \bar{k}^{-}\right)^{2}$, in accordance to the convention (3.50) used in the present paper. ${ }^{17}$ On both sides of (B.3), the two expressions add up to twice the argument of the original soft factor in the relevant formalism. After the square roots of the soft factor are combined with unsubtracted collinear matrix elements (see (3.49)), one removes the rapidity regulator in either scheme, taking $\delta^{-} \rightarrow 0$ and $\delta^{+} \rightarrow 0$ or $Y_{R} \rightarrow \infty$ and $Y_{L} \rightarrow-\infty$. The resulting distributions depend on the rapidity variable $\zeta$ or $\bar{\zeta}$.

\section{Combining SPS and DPS for different hard scales}

In this appendix, we discuss the generalisation of the scale setting discussed in section 6.5 to the case where the scales of the two DPS processes $\left(\mu_{1}\right.$ and $\left.\mu_{2}\right)$ and of the SPS process $\left(\mu_{h}\right)$ are different. In the four contributions (6.54) to the cross section, we now take

$$
\begin{aligned}
\sigma_{\mathrm{SPS}}: & f\left(x_{1}+x_{2}, \boldsymbol{Z}\right) & \sim E_{2}\left(\mu_{h} ; \mu_{Z}\right) f\left(\mu_{Z}\right), \\
\sigma_{\mathrm{DPS} / \mathrm{SPS}}: & D\left(x_{i}, \boldsymbol{y}_{+}, \boldsymbol{Z}\right) & \sim E_{3}\left(\mu_{1}, \mu_{2} \mid \mu_{h} ; \mu_{Z}\right) U\left(\mu_{Z}\right) f\left(\mu_{Z}\right), \\
\sigma_{\mathrm{SPS} / \mathrm{DPS}}: & D\left(x_{i}, \boldsymbol{y}_{-}, \boldsymbol{Z}\right) & \sim E_{3}\left(\mu_{h} \mid \mu_{1}, \mu_{2} ; \mu_{Z}\right) U^{*}\left(\mu_{Z}\right) f\left(\mu_{Z}\right), \\
\sigma_{\mathrm{DPS}}: & F\left(x_{i}, \boldsymbol{z}_{i}, \boldsymbol{y}\right) & \sim E_{4}\left(\mu_{1}, \mu_{2} \mid \mu_{1}, \mu_{2} ; \mu_{Z}\right) U\left(\mu_{Z}\right) U^{*}\left(\mu_{Z}\right) f\left(\mu_{Z}\right)+F_{\mathrm{int}}\left(\mu_{1}, \mu_{2}\right) .
\end{aligned}
$$

In $E_{3}$ and $E_{4}$, we now allow different scales for all three or four parton legs (see the remarks at the ends of sections 3.4 and 5.3). Scale arguments in the amplitude and in its

\footnotetext{
${ }^{17}$ The normalisation of $\zeta$ and $\bar{\zeta}$ is not relevant for the Collins Soper equation, but it does matter for the argument of the anomalous dimensions $\gamma_{F, a}$.
} 
complex conjugate are separated by a vertical bar "|". If only a single scale is given, such as $\mu_{Z}$ in (C.1), then all scales are taken equal. In the subtraction terms for the SPS/DPS interference, we now take

$$
\begin{array}{r}
\sigma_{\mathrm{DPS} / \mathrm{SPS}, y_{+} \rightarrow 0}: D \sim E_{3}\left(\bar{\mu}_{1}, \bar{\mu}_{2} \mid \mu_{h} ; \hat{\mu}_{h}\right) U\left(\hat{\mu}_{h}\right) E_{2}\left(\hat{\mu}_{h} ; \mu_{Z}\right) f\left(\mu_{Z}\right) \\
\quad \text { with } \hat{\mu}_{h}=p\left(\nu\left|\boldsymbol{y}_{+}\right| ; \mu_{Z}, \mu_{h}\right) \text { and } \bar{\mu}_{i}=p\left(\nu\left|\boldsymbol{y}_{+}\right| ; \mu_{i}, \mu_{h}\right), \\
\sigma_{\mathrm{SPS} / \mathrm{DPS}, y_{-} \rightarrow 0}: D \sim E_{3}\left(\mu_{h} \mid \bar{\mu}_{1}, \bar{\mu}_{2} ; \hat{\mu}_{h}\right) U^{*}\left(\hat{\mu}_{h}\right) E_{2}\left(\hat{\mu}_{h} ; \mu_{Z}\right) f\left(\mu_{Z}\right) \\
\quad \text { with } \hat{\mu}_{h}=p\left(\nu\left|\boldsymbol{y}_{-}\right| ; \mu_{Z}, \mu_{h}\right) \text { and } \bar{\mu}_{i}=p\left(\nu\left|\boldsymbol{y}_{-}\right| ; \mu_{i}, \mu_{h}\right)
\end{array}
$$

instead of (6.57). The profile scales $\hat{\mu}$ are the same as in the single-scale case of section 6.5, whilst $\bar{\mu}$ interpolates between the different hard scales. The relation (6.60) between the hard-scattering amplitudes for SPS and DPS holds for equal scales of $\alpha_{s}$ in all its terms. In all subtraction terms, the scale of $\alpha_{s}$ in the DPS amplitudes $H_{\alpha_{1} \beta_{1}}$ and $H_{\alpha_{2} \beta_{2}}$ should be taken as $\bar{\mu}_{i}$. This is for instance relevant for $g g \rightarrow H$, which involves the strong coupling already at leading order. With the choices just specified, one finds that the limiting relation (6.59) for the subtraction term still holds.

Generalising (6.61), the subtraction terms for DPS can be taken as follows:

$$
\begin{array}{ll}
\sigma_{\mathrm{DPS}, y_{-} \rightarrow 0}: & F \sim E_{4}\left(\bar{\mu}_{1}, \bar{\mu}_{2}\left|\mu_{1}, \mu_{2} ; \hat{\mu}_{1}, \hat{\mu}_{2}\right| \hat{\mu}_{h}, \hat{\mu}_{h}\right) U^{*}\left(\hat{\mu}_{h}\right) E_{3}\left(\hat{\mu}_{1}, \hat{\mu}_{2} \mid \hat{\mu}_{h} ; \mu_{Z}\right) U\left(\mu_{Z}\right) f\left(\mu_{Z}\right), \\
\sigma_{\mathrm{DPS}, y_{+} \rightarrow 0}: & F \sim E_{4}\left(\mu_{1}, \mu_{2}\left|\bar{\mu}_{1}, \bar{\mu}_{2} ; \hat{\mu}_{h}, \hat{\mu}_{h}\right| \hat{\mu}_{1}, \hat{\mu}_{2}\right) U\left(\hat{\mu}_{h}\right) E_{3}\left(\hat{\mu}_{h} \mid \hat{\mu}_{1}, \hat{\mu}_{2} ; \mu_{Z}\right) U^{*}\left(\mu_{Z}\right) f\left(\mu_{Z}\right), \\
\sigma_{\mathrm{DPS}, y_{ \pm} \rightarrow 0}: & F \sim E_{4}\left(\bar{\mu}_{1}, \bar{\mu}_{2} \mid \bar{\mu}_{1}, \bar{\mu}_{2} ; \hat{\mu}_{h}\right) U\left(\hat{\mu}_{h}\right) U^{*}\left(\hat{\mu}_{h}\right) E_{2}\left(\hat{\mu}_{h} ; \mu_{Z}\right) f\left(\mu_{Z}\right)
\end{array}
$$

with

$$
\hat{\mu}=p\left(\nu \min \left\{\left|\boldsymbol{y}_{+}\right|,\left|\boldsymbol{y}_{-}\right|\right\} ; \mu_{Z}, \mu_{h}\right) \quad \text { and } \quad \bar{\mu}_{i}=p\left(\nu \min \left\{\left|\boldsymbol{y}_{+}\right|,\left|\boldsymbol{y}_{-}\right|\right\} ; \mu_{i}, \mu_{h}\right) .
$$

For $\left|\boldsymbol{y}_{+}\right| \gg 1 / \nu$ and $\left|\boldsymbol{y}_{-}\right| \gg 1 / \nu$ one finds the limiting behaviour in (6.63), whereas all three terms in (C.3) tend to the $1 \mathrm{v} 1$ contribution to DPS if one or both of the transverse distances is of order $1 / \nu$. Overall, we thus find that the subtraction formalism can be adapted to work in the multi-scale case.

\section{Feynman rules}

In this appendix we give the Feynman rules used in our calculation of the matching coefficients in section 7.3. We compute cut graphs and thus need in particular the rules for propagators and vertices to the right of the cut (corresponding to the complex conjugate amplitude). This requires special care for the three-gluon vertex. A derivation of these rules can for instance be found in [148] for the QCD Lagrangian (but not for eikonal lines). The rules given here correspond to the conventions in the chapters 3 and 7 of [65]. In particular, the coupling constant is defined such that the covariant derivative reads $D_{\mu}=\partial_{\mu}+i g A_{\mu}^{a} t^{a}$.

The Feynman rules for the propagators and vertices arising from the QCD Lagrangian in Feynman gauge can be found in figure 12, where we use the notation

$$
V^{\mu \nu \rho}(p, q, r)=(p-q)^{\rho} g^{\mu \nu}+(q-r)^{\mu} g^{\nu \rho}+(r-p)^{\nu} g^{\rho \mu} .
$$

The four-gluon vertex is not given here since it does not appear in our calculations. 


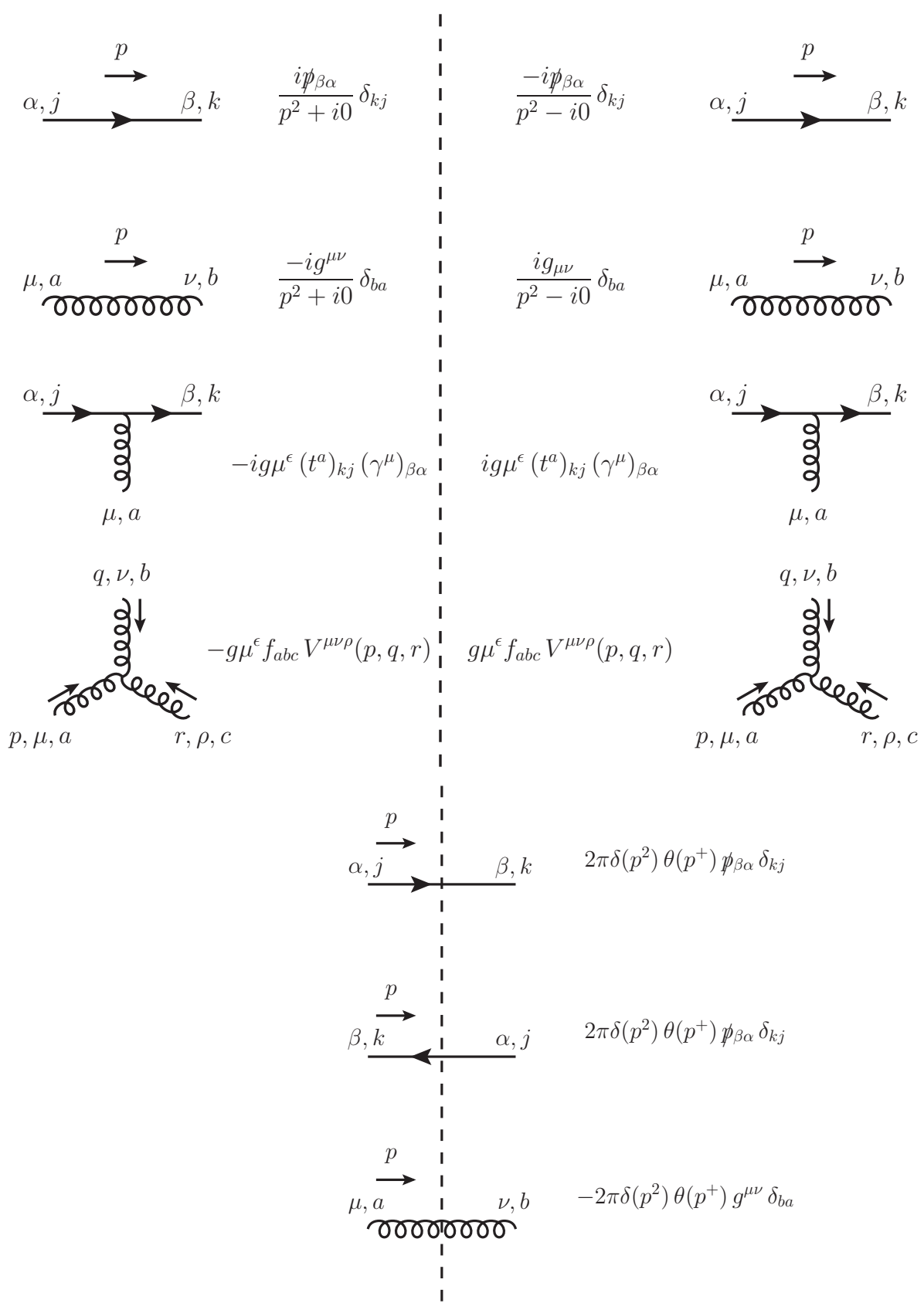

Figure 12. Feynman rules for propagators and vertices in cut graphs. We use Feynman gauge and set the quark mass to zero. $V^{\mu \nu \rho}(p, q, r)$ is defined in (D.1). With these rules for cut quark and antiquark lines, there is no minus sign for a closed fermion loop going across the cut. 


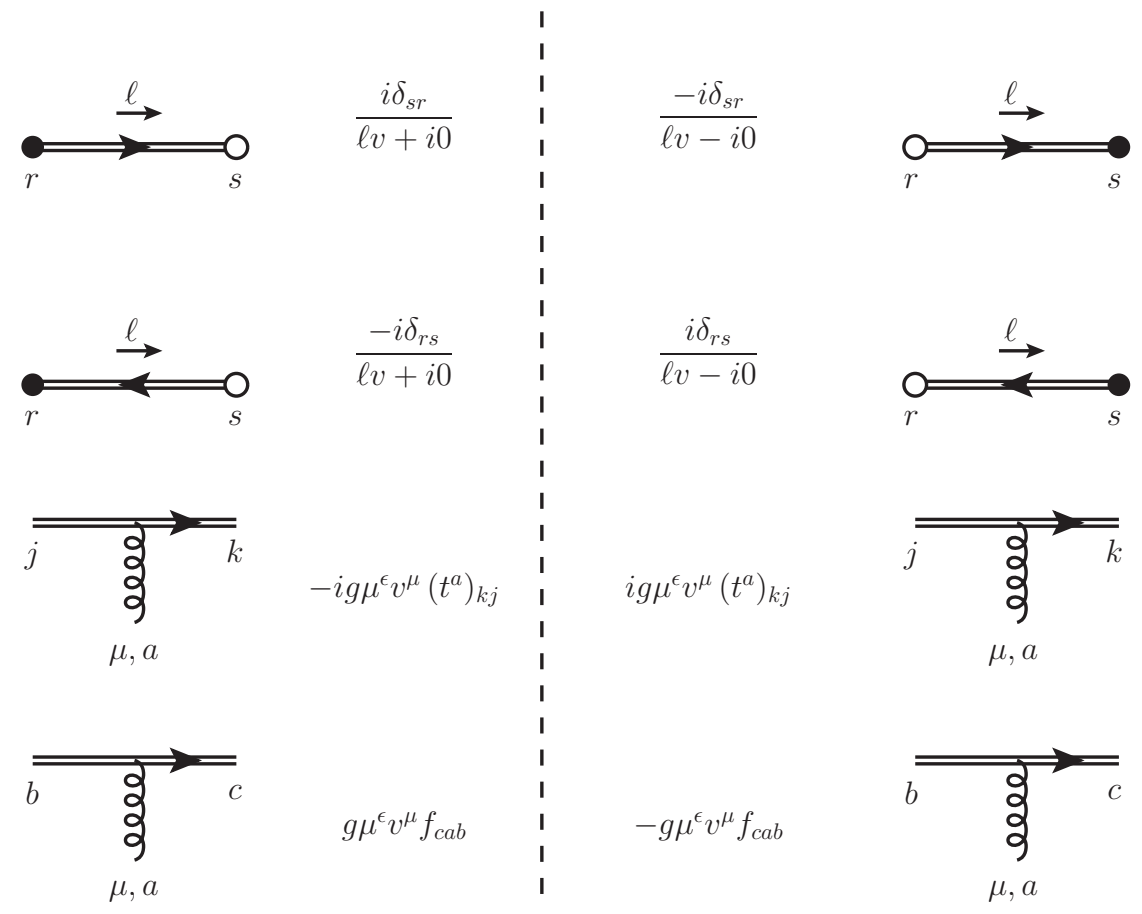

Figure 13. Feynman rules involving eikonal lines along the direction $v$. The colour indices $r$ and $s$ refer to either the fundamental or the adjoint representation, whereas $j$ and $k$ are colour triplet and $a, b$ and $c$ are colour octet indices.

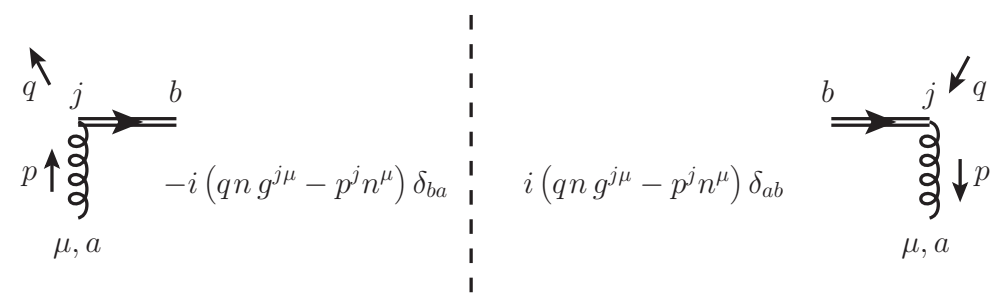

Figure 14. Feynman rule corresponding to the operator in a gluon distribution when an eikonal line along $n$ is attached to the gluon. The Lorentz index $j$ is transverse w.r.t. the lightlike direction $n$. The rule for the graph without the eikonal line is obtained by setting $p=q$.

The Feynman rules for the propagators and couplings involving eikonal lines are given in figure 13. They arise from the expansion of Wilson line operators, given by (3.5) and its analogue for the adjoint representation. We use there the notation of [27], where open and closed circles at the ends of eikonal lines (in addition to arrows on and above them) were introduced as a way to make the correspondence between graphs and mathematical expressions unique. Let us briefly explain this.

First of all, the full circle indicates the (relative) past and the open circle the (relative) future time direction when considering the path of the Wilson line in space-time. This determines the sign of $i 0$ in the eikonal propagators. Secondly, the arrow above the eikonal line propagator corresponds to the momentum flow, just as for quark and gluon propagators, and is necessary because the eikonal propagator is linear in the momentum carried 
by the line. Finally, the arrow on the eikonal line fixes the overall sign of the propagator and determines the order of contraction of colour indices, with matrix multiplication going against the direction of the arrow. This is the same convention as for Dirac indices on fermion lines. For eikonal lines in the fundamental colour representation, this arrow indicates the canonical order of multiplication, with expressions involving Gell-Mann matrices $t_{a}$ rather than their transpose $\left(t_{a}\right)^{T}$. For adjoint eikonal lines, the order in which the indices are arranged can be chosen freely (writing e.g. $f_{a b c}$ or $-f_{a c b}$ ).

The vertices for a gluon coupling to a fundamental or an adjoint eikonal line are given in the third and fourth line of figure 13, respectively. In the last row, the relative sign between the vertices to the left and the right of the cut can be understood from the relation $f_{c a b}=-i\left(T^{a}\right)_{c b}$, where $T^{a}$ acts as a generator of the colour group. The arrow on the line is required to distinguish the role of the indices $c$ and $b$, given that $f_{c a b}$ is antisymmetric in its indices. ${ }^{18}$

In figure 14 we give the Feynman rule for the operator in a gluon distribution when an eikonal line is attached to the gluon. This rule was derived in section 7.6 of [65], see also $[149,150]$. It can be obtained by expanding the operator $n_{\mu} F^{\mu j}(z) W(z, n)$ in $g$. The Lorentz index $j$ in the gluon field strength is restricted to be transverse to the lightlike vector $n$. Note that this rule corresponds to the gluon operator (3.6) only if the Wilson line is along $v_{L}=n$.

\section{E Useful integrals}

In this appendix, we give a number of Fourier integrals that are useful for the calculations in section 7.3. We start with

$$
\begin{aligned}
\int \frac{d^{2-2 \epsilon} \boldsymbol{k}}{\left(\boldsymbol{k}^{2}\right)^{\alpha}} e^{i \boldsymbol{k} \boldsymbol{z}}= & \left(\frac{\boldsymbol{z}^{2}}{4 \pi}\right)^{\epsilon+\alpha-1} \frac{\pi^{\alpha} \Gamma(1-\epsilon-\alpha)}{\Gamma(\alpha)}, \\
\int \frac{d^{2-2 \epsilon} \boldsymbol{k}}{\left(\boldsymbol{k}^{2}\right)^{\alpha}} e^{i \boldsymbol{k} \boldsymbol{z}} \log \left(\boldsymbol{k}^{2}\right)= & \left(\frac{\boldsymbol{z}^{2}}{4 \pi}\right)^{\epsilon+\alpha-1} \frac{\pi^{\alpha} \Gamma(1-\epsilon-\alpha)}{\Gamma(\alpha)} \\
& \times\left[\log \frac{4}{\boldsymbol{z}^{2}}+\psi(\alpha)+\psi(1-\alpha-\epsilon)\right],
\end{aligned}
$$

where $\psi(x)=\frac{d}{d z} \log \Gamma(z)$ is the digamma function. One can obtain (E.2) by differentiation of (E.1) with respect to $\alpha$. A useful relation for integrals involving tensors is given by

$$
\int \frac{d^{2-2 \epsilon} \boldsymbol{k}}{\left(\boldsymbol{k}^{2}\right)^{\alpha}} e^{i \boldsymbol{k} \boldsymbol{z}} \boldsymbol{k}^{j} \boldsymbol{k}^{j^{\prime}}=-\frac{\pi^{\alpha-1} \Gamma(3-\alpha-\epsilon)}{\Gamma(\alpha)}\left(\frac{\boldsymbol{z}^{2}}{4 \pi}\right)^{\epsilon+\alpha-2}\left[\frac{\boldsymbol{z}^{j} \boldsymbol{z}^{j^{\prime}}}{\boldsymbol{z}^{2}}+\frac{\delta^{j j^{\prime}}}{2(\epsilon+\alpha-2)}\right] .
$$

We obtain (E.3) by rewriting $\boldsymbol{k}^{j} \boldsymbol{k}^{j^{\prime}}$ as $-\partial^{2} /\left(\partial \boldsymbol{z}^{j} \partial \boldsymbol{z}^{j^{\prime}}\right)$ on the l.h.s. of (E.3) and then performing these derivatives on the r.h.s. of (E.1). From the above equations, it follows that

$$
\int \frac{d^{2-2 \epsilon} \boldsymbol{k}}{\boldsymbol{k}^{2}} e^{i \boldsymbol{k} \boldsymbol{z}}\left[\frac{k^{j j^{\prime}}}{\boldsymbol{k}^{2}}+f(\epsilon) \delta^{j j^{\prime}}\right]=-\pi \Gamma(1-\epsilon)\left(\frac{\boldsymbol{z}^{2}}{4 \pi}\right)^{\epsilon}\left[\frac{z^{j j^{\prime}}}{\boldsymbol{z}^{2}}+\frac{f(\epsilon)}{\epsilon} \delta^{j j^{\prime}}\right],
$$

\footnotetext{
${ }^{18}$ We note that in figure 7.10 of [65] a minus sign is missing in the coupling of a gluon to an adjoint eikonal line on the right of the cut. Otherwise, we agree with the Feynman rules given there.
} 
where the traceless symmetric tensors $k^{j j^{\prime}}$ and $z^{j j^{\prime}}$ are defined by (7.34) and $f(\epsilon)$ is an arbitrary function. The integral over $k^{j j^{\prime}} / \boldsymbol{k}^{4}$ is thus finite, whereas the one over $f(\epsilon) / \boldsymbol{k}^{2}$ has an ultraviolet pole if $f(\epsilon)$ is finite at $\epsilon=0$.

\section{F Tensor decomposition of DPDs}

In this appendix, we give the tensor decompositions of DTMDs and DPDFs in terms of scalar and pseudoscalar functions. We emphasise that these decompositions are only meant to be complete in the 2 physical transverse dimensions. In calculations that have to be done in $2-2 \epsilon$ transverse dimensions, one must either avoid the decompositions below (as we do) or extend them appropriately.

DTMDs depend on the three transverse vectors $\boldsymbol{y}, \boldsymbol{z}_{1}$ and $\boldsymbol{z}_{2}$, but one can construct the tensors necessary for their decomposition from $\boldsymbol{y}$ and

$$
\tilde{\boldsymbol{y}}^{j}=\epsilon^{j j^{\prime}} \boldsymbol{y}^{j^{\prime}}
$$

together with the invariants $\delta^{j j^{\prime}}$ and $\epsilon^{j j^{\prime}}$. This is because in 2 dimensions there are only two linearly independent vectors. The choice of the above two vectors results in the most straightforward correspondence between the DTMDs and the DPDFs, where only the vectors $\boldsymbol{y}$ and $\tilde{\boldsymbol{y}}$ can be used. The decomposition of the double quark distributions [27] reads

$$
\begin{aligned}
& F_{q q}\left(x_{i}, \boldsymbol{z}_{i}, \boldsymbol{y}\right)=f_{q q}\left(x_{i}, \boldsymbol{z}_{i}, \boldsymbol{y}\right), \\
& F_{\Delta q \Delta q}=f_{\Delta q \Delta q}, \\
& F_{\Delta q q}=g_{\Delta q q}, \\
& F_{q \Delta q}=g_{q \Delta q}, \\
& F_{\delta q q}^{j}=\tilde{\boldsymbol{y}}^{j} M f_{\delta q q}+\boldsymbol{y}^{j} M g_{\delta q q}, \\
& F_{q \delta q}^{j}=\tilde{\boldsymbol{y}}^{j} M f_{q \delta q}+\boldsymbol{y}^{j} M g_{q \delta q}, \\
& F_{\delta q \delta q}^{j j^{\prime}}=\delta^{j j^{\prime}} f_{\delta q \delta q}+2 \tau^{j j^{\prime}, \boldsymbol{y} \boldsymbol{y}} M^{2} f_{\delta q \delta q}^{t} \\
& +2 \tau^{j j^{\prime}, \boldsymbol{y} \tilde{\boldsymbol{y}}} M^{2} g_{\delta q \delta q}^{s}+\left(\boldsymbol{y}^{j} \tilde{\boldsymbol{y}}^{j^{\prime}}-\tilde{\boldsymbol{y}}^{j} \boldsymbol{y}^{j^{\prime}}\right) M^{2} g_{\delta q \delta q}^{a},
\end{aligned}
$$

where $M$ is the proton mass and the tensor $\tau^{j j^{\prime}, k k^{\prime}}$ is defined in (3.8). Note that one has $\tau^{j j^{\prime}, k k^{\prime}} \tau^{k k^{\prime}, l l^{\prime}}=\tau^{j j^{\prime}, l l^{\prime}}$. We employ a shorthand notation where vectors $\boldsymbol{y}$ or $\tilde{\boldsymbol{y}}$ appearing as an index of $\tau$ denote contraction, i.e. $\tau^{j j^{\prime}, \boldsymbol{y} y}=\tau^{j j^{\prime}, k k^{\prime}} \boldsymbol{y}^{k} \boldsymbol{y}^{k^{\prime}}$ etc. $f$ and $g$ denote scalar and pseudo-scalar functions respectively. The pseudoscalar functions are absent in the decomposition of DPDFs because one cannot construct a pseudoscalar from $\boldsymbol{y}$ alone. Decompositions analogous to (F.2) hold for quark-antiquark distributions and for distributions of two antiquarks. 
For quark-gluon distributions we write

$$
\begin{aligned}
F_{q g}\left(x_{i}, \boldsymbol{z}_{i}, \boldsymbol{y}\right) & =f_{q g}\left(x_{i}, \boldsymbol{z}_{i}, \boldsymbol{y}\right), \\
F_{\Delta q \Delta g}= & f_{\Delta q \Delta g}, \\
F_{\Delta q g}= & g_{\Delta q g}, \\
F_{q \Delta g}= & g_{q \Delta g}, \\
F_{\delta q g}^{j}= & \tilde{\boldsymbol{y}}^{j} M f_{\delta q g}+\boldsymbol{y}^{j} M g_{\delta q g}, \\
F_{q \delta g}^{j j^{\prime}}= & \tau^{j j^{\prime}, \boldsymbol{y} \boldsymbol{y}} M^{2} f_{q \delta g}+\tau^{j j^{\prime}, \boldsymbol{y} \tilde{\boldsymbol{y}}} M^{2} g_{q \delta g}, \\
F_{\delta q \delta g}^{j, k k^{\prime}}= & -\tau^{\tilde{\boldsymbol{y}} j, k k^{\prime}} M f_{\delta q \delta g}-\left(\tilde{\boldsymbol{y}}^{j} \tau^{k k^{\prime}, \boldsymbol{y} \boldsymbol{y}}+\boldsymbol{y}^{j} \tau^{k k^{\prime}, \boldsymbol{y} \tilde{\boldsymbol{y}}}\right) M^{3} f_{\delta q \delta g}^{t} \\
& -\tau^{\boldsymbol{y} j, k k^{\prime}} M g_{\delta q \delta g}-\left(\boldsymbol{y}^{j} \tau^{k k^{\prime}, \boldsymbol{y} \boldsymbol{y}}-\tilde{\boldsymbol{y}}^{j} \tau^{k k^{\prime}, \boldsymbol{y} \tilde{\boldsymbol{y}}}\right) M^{3} g_{\delta q \delta g}^{t} .
\end{aligned}
$$

Analogous decompositions hold when the quark is replaced by an antiquark. Two-gluon distributions are decomposed as

$$
\begin{aligned}
F_{g g}\left(x_{i}, \boldsymbol{z}_{i}, \boldsymbol{y}\right)= & f_{g g}\left(x_{i}, \boldsymbol{z}_{i}, \boldsymbol{y}\right), \\
F_{\Delta g \Delta g}= & f_{\Delta g \Delta g}, \\
F_{\Delta g g}= & g_{\Delta g g}, \\
F_{g \Delta g}= & g_{g \Delta g}, \\
F_{\delta g g}^{j j^{\prime}}= & \tau^{j j^{\prime}, \boldsymbol{y} \boldsymbol{y}} M^{2} f_{\delta g g}+\tau^{j j^{\prime}, \boldsymbol{y} \tilde{\boldsymbol{y}}} M^{2} g_{\delta g g}, \\
F_{g \delta g}^{j j^{\prime}}= & \tau^{j j^{\prime}, \boldsymbol{y} \boldsymbol{y}} M^{2} f_{g \delta g}+\tau^{j j^{\prime}, \boldsymbol{y} \tilde{\boldsymbol{y}}} M^{2} g_{g \delta g}, \\
F_{\delta g \delta g}^{j j^{\prime}, k k^{\prime}}= & \tau^{j j^{\prime}, k k^{\prime}} f_{\delta g \delta g} / 2+\left(\tau^{j j^{\prime}, \boldsymbol{y} \tilde{\boldsymbol{y}}} \tau^{k k^{\prime}, \boldsymbol{y} \tilde{\boldsymbol{y}}}-\tau^{j j^{\prime}, \boldsymbol{y} \boldsymbol{y}} \tau^{k k^{\prime}, \boldsymbol{y} \boldsymbol{y}}\right) M^{4} f_{\delta g \delta g}^{t} \\
& +\left(\tau^{j j^{\prime}, \boldsymbol{y} \tilde{\boldsymbol{y}}} \tau^{k k^{\prime}, \boldsymbol{y} \boldsymbol{y}}+\tau^{j j^{\prime}, \boldsymbol{y} \boldsymbol{y}} \tau^{k k^{\prime}, \boldsymbol{y} \tilde{\boldsymbol{y}}}\right) M^{4} g_{\delta g \delta g}^{s} \\
& +\left(\tau^{j j^{\prime}, \boldsymbol{y} \tilde{\boldsymbol{y}}} \tau^{k k^{\prime}, \boldsymbol{y} \boldsymbol{y}}-\tau^{j j^{\prime}, \boldsymbol{y} \boldsymbol{y}} \tau^{k k^{\prime}, \boldsymbol{y} \tilde{\boldsymbol{y}}}\right) M^{4} g_{\delta g \delta g}^{a} .
\end{aligned}
$$

We note that in the decomposition of each DTMD, the tensors multiplying different terms are orthogonal to each other.

The decompositions for the nonzero DPDFs in terms of real-valued scalar functions have already been given in $[27,33]$. They can be directly obtained from the DTMD decompositions above by setting all pseudo-scalar functions (i.e. all $g$ 's) equal to zero (and removing the arguments $\boldsymbol{z}_{i}$ in $F$ and $f$ ).

\section{G Matching coefficients for (pseudo)scalar DPDs}

Using the decompositions in the previous subsection and the results for the one-loop matching coefficients given in section 7.3.4, we can perform the necessary tensor contractions and obtain a full set of matching equations at the level of scalar and pseudoscalar distributions. We emphasise that this is done in the two physical space-time dimensions, since all intermediate calculations requiring dimensional regularisation have been completed at this point. For definiteness, let us recall that the tensor-valued coefficients in section 7.3.4 appear in 
the matching relations as

$$
\begin{aligned}
{ }^{R} F_{\delta g \delta g}^{j j^{\prime}, k k^{\prime}}\left(x_{i}, \boldsymbol{z}_{i}, \boldsymbol{y} ; \zeta\right)= & { }^{R} C_{\delta g \delta g}^{j j^{\prime}, l l^{\prime}}\left(x_{1}^{\prime}, \boldsymbol{z}_{1} ; x_{1} \zeta / x_{2}\right) \underset{x_{1}}{\otimes}{ }^{R} C_{\delta g \delta g}^{k k^{\prime}, m m^{\prime}}\left(x_{2}^{\prime}, \boldsymbol{z}_{2} ; x_{2} \zeta / x_{1}\right) \\
& \stackrel{\otimes}{ }{ }^{R} F_{\delta g \delta g}^{l l^{\prime}, m m^{\prime}}\left(x_{i}^{\prime}, \boldsymbol{y} ; \zeta\right)+\{\text { quark-gluon mixing terms }\}
\end{aligned}
$$

for the distribution $F_{\delta g \delta g}$ and in an analogous manner in other relations involving DPDs that carry polarisation indices. In an expansion up to $\mathcal{O}\left(\alpha_{s}\right)$, one keeps the one-loop terms only in one of the two matching coefficients.

In the following we give the terms with matching coefficients for the parton with momentum fraction $x_{1}$; the terms for the parton with momentum fraction $x_{2}$ are fully analogous. We omit colour labels $R$ for brevity. With the tensor structure of the kernels in section 7.3.4 we then obtain for two-quark distributions

$$
\begin{aligned}
f_{q q} & =C_{q q} \underset{x_{1}}{\otimes} f_{q q}+C_{q g} \underset{x_{1}}{\otimes} f_{g q}-\boldsymbol{y}^{2} M^{2} \omega_{1} C_{q \delta g} \underset{x_{1}}{\otimes} f_{\delta g q}, \\
f_{\Delta q \Delta q} & =C_{\Delta q \Delta q} \underset{x_{1}}{\otimes} f_{\Delta q \Delta q}+C_{\Delta q \Delta g} \underset{x_{1}}{\otimes} f_{\Delta g \Delta q}, \\
g_{\Delta q q} & =g_{q \Delta q}=0 \\
|\boldsymbol{y}| M f_{\delta q q} & =|\boldsymbol{y}| M C_{\delta q \delta q} \underset{x_{1}}{\otimes} f_{\delta q q}, \\
|\boldsymbol{y}| M g_{\delta q q} & =0 \\
|\boldsymbol{y}| M f_{q \delta q} & =|\boldsymbol{y}| M C_{q q} \underset{x_{1}}{\otimes} f_{q \delta q}+|\boldsymbol{y}| M C_{q g} \underset{x_{1}}{\otimes} f_{g \delta q}+|\boldsymbol{y}| M \omega_{1} C_{q \delta g} \underset{x_{1}}{\otimes}\left(f_{\delta g \delta q}+\boldsymbol{y}^{2} M^{2} f_{\delta g \delta q}^{t}\right), \\
|\boldsymbol{y}| M g_{q \delta q} & =-|\boldsymbol{y}| M \tilde{\omega}_{1} C_{q \delta g} \underset{x_{1}}{\otimes}\left(f_{\delta g \delta q}-\boldsymbol{y}^{2} M^{2} f_{\delta g \delta q}^{t}\right) \\
f_{\delta q \delta q} & =C_{\delta q \delta q} \underset{x_{1}}{\otimes} f_{\delta q \delta q}, \\
\boldsymbol{y}^{2} M^{2} f_{\delta q \delta q}^{t} & =\boldsymbol{y}^{2} M^{2} C_{\delta q \delta q} \underset{x_{1}}{\otimes} f_{\delta q \delta q}^{t}, \\
g_{\delta q \delta q}^{s} & =g_{\delta q \delta q}^{a}=0,
\end{aligned}
$$

where we have defined

$$
\begin{aligned}
& \omega_{1}=-\frac{2 \tau^{\boldsymbol{y} \boldsymbol{y}, \boldsymbol{z}_{1} \boldsymbol{z}_{1}}}{\boldsymbol{y}^{2} \boldsymbol{z}_{1}^{2}}=-\cos \left(2 \varphi_{1}\right), \\
& \tilde{\omega}_{1}=-\frac{2 \tau^{\boldsymbol{y} \tilde{\boldsymbol{y}}, \boldsymbol{z}_{1} \boldsymbol{z}_{1}}}{\boldsymbol{y}^{2} \boldsymbol{z}_{1}^{2}}=\sin \left(2 \varphi_{1}\right)
\end{aligned}
$$

with $\tilde{\boldsymbol{y}}$ given in (F.1). Here $\varphi_{1}$ is the angle between $\boldsymbol{y}$ and $\boldsymbol{z}_{1}$ in the transverse plane, oriented such that $\epsilon^{j j^{\prime}} \boldsymbol{y}^{j} \boldsymbol{z}_{1}^{j^{\prime}}=|\boldsymbol{y}|\left|\boldsymbol{z}_{1}\right| \sin \varphi_{1}$. For mixed quark and gluon distributions the 
matching reads

$$
\begin{aligned}
f_{g q} & =C_{g g} \underset{x_{1}}{\otimes} f_{g q}-\boldsymbol{y}^{2} M^{2} \omega_{1} C_{g \delta g} \underset{x_{1}}{\otimes} f_{\delta g q}+C_{g q} \underset{x_{1}}{\otimes} f_{q q}, \\
f_{\Delta g \Delta q} & =C_{\Delta g \Delta g} \underset{x_{1}}{\otimes} f_{\Delta g \Delta q}+C_{\Delta g \Delta q} \underset{x_{1}}{\otimes} f_{\Delta q \Delta q}, \\
g_{\Delta g q} & =g_{g \Delta q}=0 \\
\boldsymbol{y}^{2} M^{2} f_{\delta g q} & =\boldsymbol{y}^{2} M^{2} C_{\delta g \delta g} \underset{x_{1}}{\otimes} f_{\delta g q}-\omega_{1} C_{\delta g g} \underset{x_{1}}{\otimes} f_{g q}-\omega_{1} C_{\delta g q} \underset{x_{1}}{\otimes} f_{q q}, \\
\boldsymbol{y}^{2} M^{2} g_{\delta g q} & =-\tilde{\omega}_{1} C_{\delta g g} \underset{x_{1}}{\otimes} f_{g q}-\tilde{\omega}_{1} C_{\delta g q} \underset{x_{1}}{\otimes} f_{q q}, \\
|\boldsymbol{y}| M f_{g \delta q} & =|\boldsymbol{y}| M C_{g g} \underset{x_{1}}{\otimes} f_{g \delta q}+|\boldsymbol{y}| M \omega_{1} C_{g \delta g} \underset{x_{1}}{\otimes}\left(f_{\delta g \delta q}+\boldsymbol{y}^{2} M^{2} f_{\delta g \delta q}^{t}\right)+|\boldsymbol{y}| M C_{g q} \underset{x_{1}}{\otimes} f_{q \delta q}, \\
|\boldsymbol{y}| M g_{g \delta q} & =-|\boldsymbol{y}| M \tilde{\omega}_{1} C_{g \delta g} \underset{x_{1}}{\otimes}\left(f_{\delta g \delta q}-\boldsymbol{y}^{2} M^{2} f_{\delta g \delta q}^{t}\right), \\
2|\boldsymbol{y}| M f_{\delta g \delta q} & =2|\boldsymbol{y}| M C_{\delta g \delta g} \underset{x_{1}}{\otimes} f_{\delta g \delta q}+|\boldsymbol{y}| M \omega_{1} C_{\delta g g} \underset{x_{1}}{\otimes} f_{g \delta q}+|\boldsymbol{y}| M \omega_{1} C_{\delta g q} \underset{x_{1}}{\otimes} f_{q \delta q}, \\
2|\boldsymbol{y}|^{3} M^{3} f_{\delta g \delta q}^{t} & =2|\boldsymbol{y}|^{3} M{ }^{3} C_{\delta g \delta g} \underset{x_{1}}{\otimes} f_{\delta g \delta q}^{t}+|\boldsymbol{y}| M_{\omega_{1}} C_{\delta g g} \underset{x_{1}}{\otimes} f_{g \delta q}+|\boldsymbol{y}| M \omega_{1} C_{\delta g q} \underset{x_{1}}{\otimes} f_{q \delta q}, \\
2|\boldsymbol{y}| M g_{\delta g \delta q} & =-|\boldsymbol{y}| M \tilde{\omega}_{1}\left(C_{\delta g g} \underset{x_{1}}{\otimes} f_{g \delta q}+C_{\delta g q} \underset{x_{1}}{\otimes} f_{q \delta q}\right), \\
2|\boldsymbol{y}|^{3} M^{3} g_{\delta g \delta q}^{t} & =-|\boldsymbol{y}| M \tilde{\omega}_{1}\left(C_{\delta g g} \underset{x_{1}}{\otimes} f_{g \delta q}+C_{\delta g q} \underset{x_{1}}{\otimes} f_{q \delta q}\right)
\end{aligned}
$$

and

$$
\begin{aligned}
f_{q g} & =C_{q q} \underset{x_{1}}{\otimes} f_{q g}+C_{q g} \underset{x_{1}}{\otimes} f_{g g}-\boldsymbol{y}^{2} M^{2} \omega_{1} C_{q \delta g} \underset{x_{1}}{\otimes} f_{\delta g g}, \\
f_{\Delta q \Delta g} & =C_{\Delta q \Delta q} \underset{x_{1}}{\otimes} f_{\Delta q \Delta g}+C_{\Delta q \Delta g} \underset{x_{1}}{\otimes} f_{\Delta g \Delta g}, \\
g_{\Delta q g} & =g_{q \Delta g}=0 \\
|\boldsymbol{y}| M f_{\delta q g} & =|\boldsymbol{y}| M C_{\delta q \delta q} \underset{x_{1}}{\otimes} f_{\delta q g}, \\
|\boldsymbol{y}| M g_{\delta q g} & =0 \\
\boldsymbol{y}^{2} M^{2} f_{q \delta g} & =\boldsymbol{y}^{2} M^{2} C_{q q} \underset{x_{1}}{\otimes} f_{q \delta g}+\boldsymbol{y}^{2} M^{2} C_{q g} \underset{x_{1}}{\otimes} f_{g \delta g}-\omega_{1} C_{q \delta g} \underset{x_{1}}{\otimes}\left(f_{\delta g \delta g}-\boldsymbol{y}^{4} M^{4} f_{\delta g \delta g}^{t}\right), \\
\boldsymbol{y}^{2} M^{2} g_{q \delta g} & =-\tilde{\omega}_{1} C_{q \delta g} \underset{x_{1}}{\otimes}\left(f_{\delta g \delta g}+\boldsymbol{y}^{4} M^{4} f_{\delta g \delta g}^{t}\right) \\
|\boldsymbol{y}| M f_{\delta q \delta g} & =|\boldsymbol{y}| M C_{\delta q \delta q} \underset{x_{1}}{\otimes} f_{\delta q \delta g}, \\
\left|\boldsymbol{y}^{3}\right| M^{3} f_{\delta q \delta g}^{t} & =|\boldsymbol{y}|^{3} M^{3} C_{\delta q \delta q} \underset{x_{1}}{\otimes} f_{\delta q \delta g}^{t}, \\
g_{\delta q \delta g} & =g_{\delta q \delta g}^{t}=0 .
\end{aligned}
$$


For two-gluon distributions we have

$$
\begin{aligned}
f_{g g} & =C_{g g} \underset{x_{1}}{\otimes} f_{g g}-\boldsymbol{y}^{2} M^{2} \omega_{1} C_{g \delta g} \underset{x_{1}}{\otimes} f_{\delta g g}+C_{g q} \underset{x_{1}}{\otimes} f_{q g}, \\
f_{\Delta g \Delta g} & =C_{\Delta g \Delta g} \underset{x_{1}}{\otimes} f_{\Delta g \Delta g}+C_{\Delta g \Delta q} \underset{x_{1}}{\otimes} f_{\Delta q \Delta g}, \\
g_{\Delta g g} & =g_{g \Delta g}=0 \\
\boldsymbol{y}^{2} M^{2} f_{\delta g g} & =\boldsymbol{y}^{2} M^{2} C_{\delta g \delta g} \underset{x_{1}}{\otimes} f_{\delta g g}-\omega_{1} C_{\delta g g} \underset{x_{1}}{\otimes} f_{g g}-\omega_{1} C_{\delta g q} \underset{x_{1}}{\otimes} f_{q g}, \\
\boldsymbol{y}^{2} M^{2} g_{\delta g g} & =-\tilde{\omega}_{1} C_{\delta g g} \underset{x_{1}}{\otimes} f_{g g}-\tilde{\omega}_{1} C_{\delta g q} \underset{x_{1}}{\otimes} f_{q g}, \\
\boldsymbol{y}^{2} M^{2} f_{g \delta g} & =\boldsymbol{y}^{2} M^{2} C_{g g} \underset{x_{1}}{\otimes} f_{g \delta g}-\omega_{1} C_{g \delta g} \underset{x_{1}}{\otimes}\left(f_{\delta g \delta g}-\boldsymbol{y}^{4} M^{4} f_{\delta g \delta g}^{t}\right), \\
\boldsymbol{y}^{2} M^{2} g_{g \delta g} & =-\tilde{\omega}_{1} C_{g \delta g} \underset{x_{1}}{\otimes}\left(f_{\delta g \delta g}+\boldsymbol{y}^{4} M^{4} f_{\delta g \delta g}^{t}\right), \\
2 f_{\delta g \delta g} & =2 C_{\delta g \delta g} \underset{x_{1}}{\otimes} f_{\delta g \delta g}-\boldsymbol{y}^{2} M^{2} \omega_{1} C_{\delta g g} \underset{x_{1}}{\otimes} f_{g \delta g}-\boldsymbol{y}^{2} M^{2} \omega_{1} C_{\delta g q} \underset{x_{1}}{\otimes} f_{q \delta g}, \\
2 \boldsymbol{y}^{4} M^{4} f_{\delta g \delta g}^{t} & =2 \boldsymbol{y}^{4} M^{4} C_{\delta g \delta g} \underset{x_{1}}{\otimes} f_{\delta g \delta g}^{t}+\boldsymbol{y}^{2} M^{2} \omega_{1} C_{\delta g g} \underset{x_{1}}{\otimes} f_{g \delta g}+\boldsymbol{y}^{2} M^{2} \omega_{1} C_{\delta g q} \underset{x_{1}}{\otimes} f_{q \delta g}, \\
2 \boldsymbol{y}^{4} M^{4} g_{\delta g \delta g}^{s} & =-\boldsymbol{y}^{2} M^{2} \tilde{\omega}_{1} C_{\delta g g} \underset{x_{1}}{\otimes} f_{g \delta g}-\boldsymbol{y}^{2} M^{2} \tilde{\omega}_{1} C_{\delta g q} \underset{x_{1}}{\otimes} f_{q \delta g}, \\
g_{\delta g \delta g}^{a} & =g_{\delta g \delta g}^{s} .
\end{aligned}
$$

Further relations are obtained by replacing quark by antiquark labels.

Let us comment on the $\boldsymbol{y}$ dependent factors in the above relations. The factors of $|\boldsymbol{y} M|^{n}$ are such that they can be completely absorbed into the distributions (such rescaled distributions were denoted by $h$ in [33]). The factors $\omega_{1}$ and $\tilde{\omega}_{1}$ appear in the matching between DTMDs and DPDFs carrying different polarisations; the azimuthal dependence they provide is required by the conservation of angular momentum along the $z$ axis. The factor $\tilde{\omega}_{1}$ appears in the matching between pseudoscalar and scalar functions and ensures that the r.h.s. of the matching equations is odd under parity.

\section{H Splitting kernels for (pseudo)scalar DPDs}

In this appendix, we give the matching of scalar and pseudoscalar DTMDs onto PDFs in the region of small $\boldsymbol{y}$ and $\boldsymbol{z}_{i}$. The following results readily follow from the splitting kernels given in section 7.4 and the tensor decompositions in appendix F. The scalar matching kernels ${ }^{R} T_{a_{0} \rightarrow a_{1} a_{2}}$ used in the following are obtained from those in section 7.4 by removing all tensors $\delta, \epsilon$ and $\tau$, so that one has ${ }^{1} T_{g \rightarrow q \bar{q}}=T_{F}\left(u^{2}+\bar{u}^{2}\right),{ }^{1} T_{g \rightarrow \Delta q \bar{q}}=-i T_{F}(u-\bar{u})$, ${ }^{1} T_{g \rightarrow \delta q \delta \bar{q}}=-2 T_{F} u \bar{u},{ }^{1} T_{q \rightarrow \delta g q}=2 C_{F} \bar{u} / u$, etc. The only exception to this rule is that we define ${ }^{1} T_{g \rightarrow \delta g \delta g}=2 C_{A} u \bar{u}$. Then the coefficients ${ }^{1} T_{a_{0} \rightarrow a_{1} a_{2}}$ used here agree with the coefficients $T_{a_{0} \rightarrow a_{1} a_{2}}$ in appendix B of [43] for all channels. The kernels for other colour representations are obtained from (7.97). 
The matching between (pseudo)scalar DTMDs and PDFs then reads

$$
\begin{aligned}
& { }^{R} f_{\mathrm{spl}, q \bar{q}}\left(x_{i}, \boldsymbol{z}_{i}, \boldsymbol{y}\right)=\frac{\boldsymbol{y}_{+} \boldsymbol{y}_{-}}{\boldsymbol{y}_{+}^{2} \boldsymbol{y}_{-}^{2}} \frac{\alpha_{s}}{2 \pi^{2}} R T_{g \rightarrow q \bar{q}} \frac{f_{g}\left(x_{1}+x_{2}\right)}{x_{1}+x_{2}}, \\
& { }^{R} f_{\mathrm{spl}, \Delta q \Delta \bar{q}}\left(x_{i}, \boldsymbol{z}_{i}, \boldsymbol{y}\right)=\frac{\boldsymbol{y}_{+} \boldsymbol{y}_{-}}{\boldsymbol{y}_{+}^{2} \boldsymbol{y}_{-}^{2}} \frac{\alpha_{s}}{2 \pi^{2}} R^{R} T_{g \rightarrow \Delta q \Delta \bar{q}} \frac{f_{g}\left(x_{1}+x_{2}\right)}{x_{1}+x_{2}}, \\
& { }^{R} g_{\mathrm{spl}, \Delta q \bar{q}}\left(x_{i}, \boldsymbol{z}_{i}, \boldsymbol{y}\right)=\frac{\tilde{\boldsymbol{y}}\left(\boldsymbol{z}_{1}-\boldsymbol{z}_{2}\right)}{\boldsymbol{y}_{+}^{2} \boldsymbol{y}_{-}^{2}} \frac{\alpha_{s}}{2 \pi^{2}}{ }^{R} T_{g \rightarrow \Delta q \bar{q}} \frac{f_{g}\left(x_{1}+x_{2}\right)}{x_{1}+x_{2}}, \\
& { }^{R} g_{\mathrm{spl}, q \Delta \bar{q}}\left(x_{i}, \boldsymbol{z}_{i}, \boldsymbol{y}\right)=\frac{\tilde{\boldsymbol{y}}\left(\boldsymbol{z}_{1}-\boldsymbol{z}_{2}\right)}{\boldsymbol{y}_{+}^{2} \boldsymbol{y}_{-}^{2}} \frac{\alpha_{s}}{2 \pi^{2}} R^{R} T_{g \rightarrow q \Delta \bar{q}} \frac{f_{g}\left(x_{1}+x_{2}\right)}{x_{1}+x_{2}}, \\
& R_{f_{\mathrm{spl}, \delta q \delta \bar{q}}}\left(x_{i}, \boldsymbol{z}_{i}, \boldsymbol{y}\right)=\frac{\boldsymbol{y}_{+} \boldsymbol{y}_{-}}{\boldsymbol{y}_{+}^{2} \boldsymbol{y}_{-}^{2}} \frac{\alpha_{s}}{2 \pi^{2}} R T_{g \rightarrow \delta q \delta \bar{q}} \frac{f_{g}\left(x_{1}+x_{2}\right)}{x_{1}+x_{2}}
\end{aligned}
$$

for quark-antiquark DTMDs. For splitting into gluon-quark distributions we have

$$
\begin{aligned}
& { }^{R} f_{\mathrm{spl}, g q}\left(x_{i}, \boldsymbol{z}_{i}, \boldsymbol{y}\right)=\frac{\boldsymbol{y}_{+} \boldsymbol{y}_{-}}{\boldsymbol{y}_{+}^{2} \boldsymbol{y}_{-}^{2}} \frac{\alpha_{s}}{2 \pi^{2}} R_{T_{q \rightarrow g q}} \frac{f_{q}\left(x_{1}+x_{2}\right)}{x_{1}+x_{2}}, \\
& { }^{R} f_{\mathrm{spl}, \Delta g \Delta q}\left(x_{i}, \boldsymbol{z}_{i}, \boldsymbol{y}\right)=\frac{\boldsymbol{y}_{+} \boldsymbol{y}_{-}}{\boldsymbol{y}_{+}^{2} \boldsymbol{y}_{-}^{2}} \frac{\alpha_{s}}{2 \pi^{2}} R_{T_{q \rightarrow \Delta g \Delta q}} \frac{f_{q}\left(x_{1}+x_{2}\right)}{x_{1}+x_{2}}, \\
& { }^{R} g_{\mathrm{spl}, \Delta g q}\left(x_{i}, \boldsymbol{z}_{i}, \boldsymbol{y}\right)=\frac{\tilde{\boldsymbol{y}}\left(\boldsymbol{z}_{1}-\boldsymbol{z}_{2}\right)}{\boldsymbol{y}_{+}^{2} \boldsymbol{y}_{-}^{2}} \frac{\alpha_{s}}{2 \pi^{2}}{ }^{R} T_{q \rightarrow \Delta g q} \frac{f_{q}\left(x_{1}+x_{2}\right)}{x_{1}+x_{2}}, \\
& { }^{R} g_{\mathrm{spl}, g \Delta q}\left(x_{i}, \boldsymbol{z}_{i}, \boldsymbol{y}\right)=\frac{\tilde{\boldsymbol{y}}\left(\boldsymbol{z}_{1}-\boldsymbol{z}_{2}\right)}{\boldsymbol{y}_{+}^{2} \boldsymbol{y}_{-}^{2}} \frac{\alpha_{s}}{2 \pi^{2}}{ }^{R} T_{q \rightarrow g \Delta q} \frac{f_{q}\left(x_{1}+x_{2}\right)}{x_{1}+x_{2}}, \\
& \left(\boldsymbol{y}^{2} M^{2}\right)^{R} f_{\mathrm{spl}, \delta g q}\left(x_{i}, \boldsymbol{z}_{i}, \boldsymbol{y}\right)=\frac{2\left(\boldsymbol{y}_{+} \boldsymbol{y}\right)\left(\boldsymbol{y}_{-} \boldsymbol{y}\right)-\boldsymbol{y}^{2}\left(\boldsymbol{y}_{+} \boldsymbol{y}_{-}\right)}{\boldsymbol{y}_{+}^{2} \boldsymbol{y}_{-}^{2}} \frac{1}{\boldsymbol{y}^{2}} \frac{\alpha_{s}}{2 \pi^{2}} R_{T_{q \rightarrow \delta g q}} \frac{f_{q}\left(x_{1}+x_{2}\right)}{x_{1}+x_{2}}, \\
& \left(\boldsymbol{y}^{2} M^{2}\right)^{R} g_{\mathrm{spl}, \delta g q}\left(x_{i}, \boldsymbol{z}_{i}, \boldsymbol{y}\right)=\frac{\left(\boldsymbol{y}_{+} \boldsymbol{y}\right)\left(\boldsymbol{y}_{-} \tilde{\boldsymbol{y}}\right)+\left(\boldsymbol{y}_{-} \boldsymbol{y}\right)\left(\boldsymbol{y}_{+} \tilde{\boldsymbol{y}}\right)}{\boldsymbol{y}_{+}^{2} \boldsymbol{y}_{-}^{2}} \frac{1}{\boldsymbol{y}^{2}} \frac{\alpha_{s}}{2 \pi^{2}}{ }^{R} T_{q \rightarrow \delta g q} \frac{f_{q}\left(x_{1}+x_{2}\right)}{x_{1}+x_{2}}
\end{aligned}
$$

and for two-gluon DTMDs

$$
\begin{aligned}
{ }_{f_{\mathrm{spl}, g g}}\left(x_{i}, \boldsymbol{z}_{i}, \boldsymbol{y}\right) & =\frac{\boldsymbol{y}_{+} \boldsymbol{y}_{-}}{\boldsymbol{y}_{+}^{2} \boldsymbol{y}_{-}^{2}} \frac{\alpha_{s}}{2 \pi^{2}} R_{T_{g \rightarrow g g}} \frac{f_{g}\left(x_{1}+x_{2}\right)}{x_{1}+x_{2}}, \\
{ }^{R} f_{\mathrm{spl}, \Delta g \Delta g}\left(x_{i}, \boldsymbol{z}_{i}, \boldsymbol{y}\right) & =\frac{\boldsymbol{y}_{+} \boldsymbol{y}_{-}}{\boldsymbol{y}_{+}^{2} \boldsymbol{y}_{-}^{2}} \frac{\alpha_{s}}{2 \pi^{2}} R_{T_{g \rightarrow \Delta g \Delta g}} \frac{f_{g}\left(x_{1}+x_{2}\right)}{x_{1}+x_{2}}, \\
{ }^{R} g_{\mathrm{spl}, g \Delta g}\left(x_{i}, \boldsymbol{z}_{i}, \boldsymbol{y}\right) & =\frac{\tilde{\boldsymbol{y}}\left(\boldsymbol{z}_{1}-\boldsymbol{z}_{2}\right)}{\boldsymbol{y}_{+}^{2} \boldsymbol{y}_{-}^{2}} \frac{\alpha_{s}}{2 \pi^{2}} R_{T_{g \rightarrow g \Delta g}} \frac{f_{g}\left(x_{1}+x_{2}\right)}{x_{1}+x_{2}}, \\
\left(\boldsymbol{y}^{2} M^{2}\right)^{R} f_{\mathrm{spl}, g \delta g}\left(x_{i}, \boldsymbol{z}_{i}, \boldsymbol{y}\right) & =\frac{2\left(\boldsymbol{y}_{+} \boldsymbol{y}\right)\left(\boldsymbol{y}_{-} \boldsymbol{y}\right)-\boldsymbol{y}^{2}\left(\boldsymbol{y}_{+} \boldsymbol{y}_{-}\right)}{\boldsymbol{y}_{+}^{2} \boldsymbol{y}_{-}^{2}} \frac{1}{\boldsymbol{y}^{2}} \frac{\alpha_{s}}{2 \pi^{2}}{ }^{R} T_{g \rightarrow g \delta g} \frac{f_{g}\left(x_{1}+x_{2}\right)}{x_{1}+x_{2}}, \\
\left(\boldsymbol{y}^{2} M^{2}\right)^{R} g_{\mathrm{spl}, g \delta g}\left(x_{i}, \boldsymbol{z}_{i}, \boldsymbol{y}\right) & =\frac{\left(\boldsymbol{y}_{+} \boldsymbol{y}\right)\left(\boldsymbol{y}_{-} \tilde{\boldsymbol{y}}\right)+\left(\boldsymbol{y}_{-} \boldsymbol{y}\right)\left(\boldsymbol{y}_{+} \tilde{\boldsymbol{y}}\right)}{\boldsymbol{y}_{+}^{2} \boldsymbol{y}_{-}^{2}} \frac{1}{\boldsymbol{y}^{2}} \frac{\alpha_{s}}{2 \pi^{2}}{ }^{R} T_{g \rightarrow g \delta g} \frac{f_{g}\left(x_{1}+x_{2}\right)}{x_{1}+x_{2}}, \\
{ }^{R} f_{\mathrm{spl}, \delta g \delta g}\left(x_{i}, \boldsymbol{z}_{i}, \boldsymbol{y}\right) & =\frac{\boldsymbol{y}_{+} \boldsymbol{y}_{-}}{\boldsymbol{y}_{+}^{2} \boldsymbol{y}_{-}^{2}} \frac{\alpha_{s}}{2 \pi^{2}} R^{R} T_{g \rightarrow \delta g \delta g} \frac{f_{g}\left(x_{1}+x_{2}\right)}{x_{1}+x_{2}} .
\end{aligned}
$$

Open Access. This article is distributed under the terms of the Creative Commons Attribution License (CC-BY 4.0), which permits any use, distribution and reproduction in any medium, provided the original author(s) and source are credited. 


\section{References}

[1] Axial Field Spectrometer collaboration, T. Akesson et al., Double parton scattering in pp collisions at $\sqrt{s}=63 \mathrm{GeV}, \mathrm{Z}$. Phys. C 34 (1987) 163 [inSPIRE].

[2] UA2 collaboration, J. Alitti et al., A study of multi-jet events at the CERN $\bar{p} p$ collider and a search for double parton scattering, Phys. Lett. B 268 (1991) 145 [INSPIRE].

[3] CDF collaboration, F. Abe et al., Study of four jet events and evidence for double parton interactions in p $\bar{p}$ collisions at $\sqrt{s}=1.8 \mathrm{TeV}$, Phys. Rev. D 47 (1993) 4857 [InSPIRE].

[4] CDF collaboration, F. Abe et al., Measurement of double parton scattering in $\bar{p} p$ collisions at $\sqrt{s}=1.8$ TeV, Phys. Rev. Lett. 79 (1997) 584 [INSPIRE].

[5] CDF collaboration, F. Abe et al., Double parton scattering in $\bar{p} p$ collisions at $\sqrt{s}=1.8$ TeV, Phys. Rev. D 56 (1997) 3811 [INSPIRE].

[6] D0 collaboration, V.M. Abazov et al., Double parton interactions in $\gamma+3$ jet events in p$p \bar{p}$ collisions $\sqrt{s}=1.96$ TeV., Phys. Rev. D 81 (2010) 052012 [arXiv:0912.5104] [INSPIRE].

[7] D0 collaboration, V.M. Abazov et al., Azimuthal decorrelations and multiple parton interactions in $\gamma+2$ jet and $\gamma+3$ jet events in $p \bar{p}$ collisions at $\sqrt{s}=1.96 \mathrm{TeV}$, Phys. Rev. D 83 (2011) 052008 [arXiv:1101.1509] [InSPIRE].

[8] D0 collaboration, V.M. Abazov et al., Double parton interactions in $\gamma+3$ jet and $\gamma+b / c$ jet +2 jet events in $p \bar{p}$ collisions at $\sqrt{s}=1.96$ TeV, Phys. Rev. D 89 (2014) 072006 [arXiv: 1402.1550] [INSPIRE].

[9] D0 collaboration, V.M. Abazov et al., Observation and studies of double $J / \psi$ production at the Tevatron, Phys. Rev. D 90 (2014) 111101 [arXiv:1406.2380] [InSPIRE].

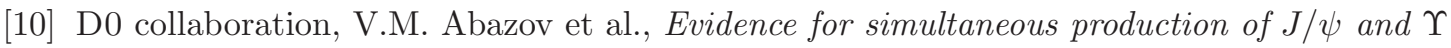
mesons, Phys. Rev. Lett. 116 (2016) 082002 [arXiv:1511.02428] [InSPIRE].

[11] D0 collaboration, V.M. Abazov et al., Study of double parton interactions in diphoton + dijet events in $p \bar{p}$ collisions at $\sqrt{s}=1.96$ TeV, Phys. Rev. D 93 (2016) 052008 [arXiv: 1512.05291] [INSPIRE].

[12] LHCb collaboration, Observation of $J / \psi$ pair production in pp collisions at $\sqrt{s}=7 \mathrm{Te}$, Phys. Lett. B 707 (2012) 52 [arXiv:1109.0963] [INSPIRE].

[13] LHCb collaboration, Observation of double charm production involving open charm in pp collisions at $\sqrt{s}=7 \mathrm{TeV}$, JHEP 06 (2012) 141 [arXiv:1205.0975] [INSPIRE].

[14] LHCb collaboration, Production of associated $\Upsilon$ and open charm hadrons in pp collisions at $\sqrt{s}=7$ and 8 TeV via double parton scattering, JHEP 07 (2016) 052 [arXiv: 1510.05949] [INSPIRE].

[15] ATLAS collaboration, Measurement of hard double-parton interactions in $W(\rightarrow \ell \nu)+2$ jet events at $\sqrt{s}=7$ TeV with the ATLAS detector, New J. Phys. 15 (2013) 033038 [arXiv:1301.6872] [INSPIRE].

[16] ATLAS collaboration, Measurement of the production cross section of prompt $J / \psi$ mesons in association with a $W^{ \pm}$boson in pp collisions at $\sqrt{s}=7$ TeV with the ATLAS detector, JHEP 04 (2014) 172 [arXiv:1401.2831] [INSPIRE].

[17] ATLAS collaboration, Observation and measurements of the production of prompt and non-prompt $J / \psi$ mesons in association with a $Z$ boson in pp collisions at $\sqrt{s}=8$ TeV with the ATLAS detector, Eur. Phys. J. C 75 (2015) 229 [arXiv:1412.6428] [INSPIRE]. 
[18] ATLAS collaboration, Study of hard double-parton scattering in four-jet events in pp collisions at $\sqrt{s}=7$ TeV with the ATLAS experiment, JHEP 11 (2016) 110 [arXiv: 1608.01857] [INSPIRE].

[19] ATLAS collaboration, Measurement of the prompt $J / \psi$ pair production cross-section in $p p$ collisions at $\sqrt{s}=8 \mathrm{TeV}$ with the ATLAS detector, Eur. Phys. J. C 77 (2017) 76 [arXiv: 1612.02950] [INSPIRE].

[20] CMS collaboration, Study of double parton scattering using $W+2$-jet events in proton-proton collisions at $\sqrt{s}=7 \mathrm{TeV}$, JHEP 03 (2014) 032 [arXiv:1312.5729] [INSPIRE].

[21] CMS collaboration, Observation of $\Upsilon(1 S)$ pair production in proton-proton collisions at $\sqrt{s}=8 \mathrm{TeV}$, JHEP 05 (2017) 013 [arXiv: 1610.07095] [INSPIRE].

[22] CMS collaboration, Measurement of double parton scattering in same-sign WW production in pp collisions at $\sqrt{s}=13 \mathrm{TeV}$ with the CMS experiment, CMS-PAS-FSQ-16-009, CERN, Geneva Switzerland, (2016).

[23] T. Sjöstrand and P.Z. Skands, Multiple interactions and the structure of beam remnants, JHEP 03 (2004) 053 [hep-ph/0402078] [INSPIRE].

[24] N. Paver and D. Treleani, Multi-quark scattering and large $p_{T}$ jet production in hadronic collisions, Nuovo Cim. A 70 (1982) 215 [INSPIRE].

[25] M. Mekhfi, Multiparton processes: an application to double Drell-Yan, Phys. Rev. D 32 (1985) 2371 [INSPIRE].

[26] M. Diehl and A. Schäfer, Theoretical considerations on multiparton interactions in QCD, Phys. Lett. B 698 (2011) 389 [arXiv:1102.3081] [InSPIRE].

[27] M. Diehl, D. Ostermeier and A. Schäfer, Elements of a theory for multiparton interactions in QCD, JHEP 03 (2012) 089 [Erratum ibid. 03 (2016) 001] [arXiv:1111.0910] [INSPIRE].

[28] A.V. Manohar and W.J. Waalewijn, A QCD analysis of double parton scattering: color correlations, interference effects and evolution, Phys. Rev. D 85 (2012) 114009 [arXiv:1202.3794] [INSPIRE].

[29] M. Diehl, J.R. Gaunt, D. Ostermeier, P. Plößl and A. Schäfer, Cancellation of Glauber gluon exchange in the double Drell-Yan process, JHEP 01 (2016) 076 [arXiv:1510.08696] [INSPIRE].

[30] M. Diehl, J.R. Gaunt and K. Schönwald, Double hard scattering without double counting, JHEP 06 (2017) 083 [arXiv:1702.06486] [INSPIRE].

[31] A.V. Manohar and W.J. Waalewijn, What is double parton scattering?, Phys. Lett. B 713 (2012) 196 [arXiv:1202.5034] [InSPIRE].

[32] M. Mekhfi, Correlations in color and spin in multiparton processes, Phys. Rev. D 32 (1985) 2380 [InSPIRE].

[33] M. Diehl and T. Kasemets, Positivity bounds on double parton distributions, JHEP 05 (2013) 150 [arXiv:1303.0842] [INSPIRE].

[34] T. Kasemets and P.J. Mulders, Constraining double parton correlations and interferences, Phys. Rev. D 91 (2015) 014015 [arXiv:1411.0726] [INSPIRE].

[35] H.-M. Chang, A.V. Manohar and W.J. Waalewijn, Double parton correlations in the bag model, Phys. Rev. D 87 (2013) 034009 [arXiv:1211.3132] [INSPIRE]. 
[36] M. Rinaldi, S. Scopetta and V. Vento, Double parton correlations in constituent quark models, Phys. Rev. D 87 (2013) 114021 [arXiv:1302.6462] [INSPIRE].

[37] M. Rinaldi, S. Scopetta, M. Traini and V. Vento, Double parton correlations and constituent quark models: a light front approach to the valence sector, JHEP 12 (2014) 028 [arXiv:1409.1500] [INSPIRE].

[38] M. Rinaldi, S. Scopetta, M.C. Traini and V. Vento, Correlations in double parton distributions: perturbative and non-perturbative effects, JHEP 10 (2016) 063 [arXiv: 1608.02521] [INSPIRE].

[39] M. Rinaldi and F.A. Ceccopieri, Relativistic effects in model calculations of double parton distribution function, Phys. Rev. D 95 (2017) 034040 [arXiv: 1611.04793] [INSPIRE].

[40] W. Broniowski and E. Ruiz Arriola, Valence double parton distributions of the nucleon in a simple model, Few Body Syst. 55 (2014) 381 [arXiv: 1310.8419] [INSPIRE].

[41] W. Broniowski, E. Ruiz Arriola and K. Golec-Biernat, Generalized Valon model for double parton distributions, Few Body Syst. 57 (2016) 405 [arXiv: 1602.00254] [INSPIRE].

[42] T. Kasemets and A. Mukherjee, Quark-gluon double parton distributions in the light-front dressed quark model, Phys. Rev. D 94 (2016) 074029 [arXiv: 1606.05686] [INSPIRE].

[43] M. Diehl, T. Kasemets and S. Keane, Correlations in double parton distributions: effects of evolution, JHEP 05 (2014) 118 [arXiv:1401.1233] [INSPIRE].

[44] T. Kasemets and M. Diehl, Angular correlations in the double Drell-Yan process, JHEP 01 (2013) 121 [arXiv:1210.5434] [INSPIRE].

[45] M.G. Echevarría, T. Kasemets, P.J. Mulders and C. Pisano, Polarization effects in double open-charm production at LHCb, JHEP 04 (2015) 034 [arXiv:1501.07291] [INSPIRE].

[46] J.R. Gaunt, Single perturbative splitting diagrams in double parton scattering, JHEP 01 (2013) 042 [arXiv: 1207.0480] [INSPIRE].

[47] B. Blok, Yu. Dokshitser, L. Frankfurt and M. Strikman, $p Q C D$ physics of multiparton interactions, Eur. Phys. J. C 72 (2012) 1963 [arXiv:1106.5533] [InSPIRE].

[48] B. Blok, Yu. Dokshitzer, L. Frankfurt and M. Strikman, Perturbative QCD correlations in multi-parton collisions, Eur. Phys. J. C 74 (2014) 2926 [arXiv:1306.3763] [InSPIRE].

[49] M.G. Ryskin and A.M. Snigirev, A fresh look at double parton scattering, Phys. Rev. D 83 (2011) 114047 [arXiv:1103.3495] [inSPIRE].

[50] M.G. Ryskin and A.M. Snigirev, Double parton scattering in double logarithm approximation of perturbative QCD, Phys. Rev. D 86 (2012) 014018 [arXiv:1203.2330] [INSPIRE].

[51] B. Blok and P. Gunnellini, Dynamical approach to MPI four-jet production in PYTHIA, Eur. Phys. J. C 75 (2015) 282 [arXiv: 1503.08246] [InSPIRE].

[52] B. Blok and P. Gunnellini, Dynamical approach to MPI in $W+$ dijet and $Z+$ dijet production within the PYTHIA event generator, Eur. Phys. J. C 76 (2016) 202 [arXiv:1510.07436] [INSPIRE].

[53] B. Blok, Yu. Dokshitzer, L. Frankfurt and M. Strikman, The four jet production at LHC and Tevatron in QCD, Phys. Rev. D 83 (2011) 071501 [arXiv:1009.2714] [InSPIRE].

[54] A. Kulesza and W.J. Stirling, Like sign $W$ boson production at the LHC as a probe of double parton scattering, Phys. Lett. B 475 (2000) 168 [hep-ph/9912232] [INSPIRE]. 
[55] J.R. Gaunt, C.-H. Kom, A. Kulesza and W.J. Stirling, Same-sign W pair production as a probe of double parton scattering at the LHC, Eur. Phys. J. C 69 (2010) 53 [arXiv: 1003.3953] [INSPIRE].

[56] F.A. Ceccopieri, M. Rinaldi and S. Scopetta, Parton correlations in same-sign W pair production via double parton scattering at the LHC, Phys. Rev. D 95 (2017) 114030 [arXiv:1702.05363] [INSPIRE].

[57] J.C. Collins, D.E. Soper and G.F. Sterman, Transverse momentum distribution in Drell-Yan pair and $W$ and $Z$ boson production, Nucl. Phys. B 250 (1985) 199 [InSPIRE].

[58] S. Catani, D. de Florian and M. Grazzini, Universality of nonleading logarithmic contributions in transverse momentum distributions, Nucl. Phys. B 596 (2001) 299 [hep-ph/0008184] [INSPIRE].

[59] S. Catani, L. Cieri, D. de Florian, G. Ferrera and M. Grazzini, Universality of transverse-momentum resummation and hard factors at the $N N L O$,

Nucl. Phys. B 881 (2014) 414 [arXiv:1311.1654] [inSPIRE].

[60] M.G. Echevarría, A. Idilbi and I. Scimemi, Factorization theorem for Drell-Yan at low $q_{T}$ and transverse momentum distributions on-the-light-cone, JHEP 07 (2012) 002 [arXiv:1111.4996] [INSPIRE].

[61] T. Becher, M. Neubert and D. Wilhelm, Higgs-boson production at small transverse momentum, JHEP 05 (2013) 110 [arXiv:1212.2621] [INSPIRE].

[62] D. Neill, I.Z. Rothstein and V. Vaidya, The Higgs transverse momentum distribution at NNLL and its theoretical errors, JHEP 12 (2015) 097 [arXiv:1503.00005] [INSPIRE].

[63] Y.L. Dokshitzer, D. Diakonov and S.I. Troian, Hard processes in quantum chromodynamics, Phys. Rept. 58 (1980) 269 [INSPIRE].

[64] J.C. Collins and D.E. Soper, Back-to-back jets in QCD, Nucl. Phys. B 193 (1981) 381 [Erratum ibid. B 213 (1983) 545] [INSPIRE].

[65] J. Collins, Foundations of perturbative QCD, Cambridge University Press, Cambridge U.K., (2011) [INSPIRE].

[66] S.M. Aybat and T.C. Rogers, TMD parton distribution and fragmentation functions with QCD evolution, Phys. Rev. D 83 (2011) 114042 [arXiv:1101.5057] [InSPIRE].

[67] J. Collins, New definition of TMD parton densities, Int. J. Mod. Phys. Conf. Ser. 4 (2011) 85 [arXiv:1107.4123] [InSPIRE].

[68] J. Collins and T.C. Rogers, Connecting different TMD factorization formalisms in QCD, Phys. Rev. D 96 (2017) 054011 [arXiv: 1705.07167] [INSPIRE].

[69] M.G.A. Buffing, M. Diehl and T. Kasemets, Double parton scattering for perturbative transverse momenta, DESY-16-201, (2016) [PoS (QCDEV2016) 015] [arXiv:1611.00178] [INSPIRE].

[70] M.G.A. Buffing, Framework for evolution in double parton scattering, PoS(DIS2017)181 [arXiv: 1707.00894] [INSPIRE].

[71] T.C. Rogers and P.J. Mulders, No generalized TMD-factorization in hadro-production of high transverse momentum hadrons, Phys. Rev. D 81 (2010) 094006 [arXiv:1001.2977] [INSPIRE]. 
[72] J.C. Collins and D.E. Soper, Back-to-back jets: Fourier transform from $b$ to $K_{\mathrm{T}}$, Nucl. Phys. B 197 (1982) 446 [inSPIRE].

[73] K. Golec-Biernat and A.M. Staśto, Unintegrated double parton distributions, Phys. Rev. D 95 (2017) 034033 [arXiv:1611.02033] [INSPIRE].

[74] A. Vladimirov, Soft factors for double parton scattering at NNLO, JHEP 12 (2016) 038 [arXiv: 1608.04920] [INSPIRE].

[75] A. Vladimirov, Structure of rapidity divergences in soft factors, arXiv:1707.07606 [INSPIRE].

[76] J.C. Collins, Renormalization: an introduction to renormalization, the renormalization group, and the operator-product expansion, Cambridge University Press, Cambridge U.K., (1984) [INSPIRE].

[77] M.E. Peskin and D.V. Schroeder, An introduction to quantum field theory, Addison-Wesley, Reading U.S.A., (1995) [INSPIRE].

[78] M.G. Echevarria, I. Scimemi and A. Vladimirov, Universal transverse momentum dependent soft function at NNLO, Phys. Rev. D 93 (2016) 054004 [arXiv:1511.05590] [INSPIRE].

[79] J.C. Collins and T.C. Rogers, Equality of two definitions for transverse momentum dependent parton distribution functions, Phys. Rev. D 87 (2013) 034018 [arXiv:1210.2100] [INSPIRE].

[80] J. Bartels, Unitarity corrections to the Lipatov Pomeron and the four gluon operator in deep inelastic scattering in QCD, Z. Phys. C 60 (1993) 471 [INSPIRE].

[81] S. Keppeler and M. Sjödahl, Orthogonal multiplet bases in $\mathrm{SU}\left(N_{c}\right)$ color space, JHEP 09 (2012) 124 [arXiv:1207.0609] [InSPIRE].

[82] P. Cvitanović, Group theory: birdtracks, Lie's, and exceptional groups, http://birdtracks.eu, Princeton Univ. Press, Princeton U.S.A., (2008) [INSPIRE].

[83] A.J. MacFarlane, A. Sudbery and P.H. Weisz, On Gell-Mann's $\lambda$-matrices, $d$ - and $f$-tensors, octets and parametrizations of SU(3), Commun. Math. Phys. 11 (1968) 77 [INSPIRE].

[84] C.J. Bomhof, P.J. Mulders and F. Pijlman, The construction of gauge-links in arbitrary hard processes, Eur. Phys. J. C 47 (2006) 147 [hep-ph/0601171] [INSPIRE].

[85] M.G. Echevarria, T. Kasemets, P.J. Mulders and C. Pisano, QCD evolution of (un)polarized gluon TMDPDFs and the Higgs $q_{T}$-distribution, JHEP 07 (2015) 158 [Erratum ibid. 05 (2017) 073] [arXiv: 1502.05354] [INSPIRE].

[86] X.-D. Ji, J.-P. Ma and F. Yuan, QCD factorization for semi-inclusive deep-inelastic scattering at low transverse momentum, Phys. Rev. D 71 (2005) 034005 [hep-ph/0404183] [INSPIRE].

[87] R. Kirschner, Generalized Lipatov-Altarelli-Parisi equations and jet calculus rules, Phys. Lett. B 84 (1979) 266 [INSPIRE].

[88] V.P. Shelest, A.M. Snigirev and G.M. Zinovev, The multiparton distribution equations in QCD, Phys. Lett. B 113 (1982) 325 [InSPIRE].

[89] A.M. Snigirev, Double parton distributions in the leading logarithm approximation of perturbative QCD, Phys. Rev. D 68 (2003) 114012 [hep-ph/0304172] [INSPIRE]. 
[90] J.R. Gaunt and W.J. Stirling, Double parton distributions incorporating perturbative QCD evolution and momentum and quark number sum rules, JHEP 03 (2010) 005 [arXiv:0910.4347] [INSPIRE].

[91] F.A. Ceccopieri, An update on the evolution of double parton distributions, Phys. Lett. B 697 (2011) 482 [arXiv:1011.6586] [InSPIRE].

[92] M. Mekhfi and X. Artru, Sudakov suppression of color correlations in multiparton scattering, Phys. Rev. D 37 (1988) 2618 [INSPIRE].

[93] A. Bacchetta, M.G. Echevarria, P.J.G. Mulders, M. Radici and A. Signori, Effects of TMD evolution and partonic flavor on $e^{+} e^{-}$annihilation into hadrons, JHEP 11 (2015) 076 [arXiv: 1508.00402] [INSPIRE].

[94] T. Becher, M. Neubert and D. Wilhelm, Electroweak gauge-boson production at small $q_{T}$ : infrared safety from the collinear anomaly, JHEP 02 (2012) 124 [arXiv:1109.6027] [INSPIRE].

[95] U. D'Alesio, M.G. Echevarria, S. Melis and I. Scimemi, Non-perturbative QCD effects in $q_{T}$ spectra of Drell-Yan and Z-boson production, JHEP 11 (2014) 098 [arXiv:1407.3311] [INSPIRE].

[96] M.A. Ebert and F.J. Tackmann, Resummation of transverse momentum distributions in distribution space, JHEP 02 (2017) 110 [arXiv:1611.08610] [INSPIRE].

[97] C.A. Aidala, B. Field, L.P. Gamberg and T.C. Rogers, Limits on transverse momentum dependent evolution from semi-inclusive deep inelastic scattering at moderate $Q$,

Phys. Rev. D 89 (2014) 094002 [arXiv:1401.2654] [inSPIRE].

[98] J. Collins and T. Rogers, Understanding the large-distance behavior of transverse-momentum-dependent parton densities and the Collins-Soper evolution kernel, Phys. Rev. D 91 (2015) 074020 [arXiv:1412.3820] [INSPIRE].

[99] M.G. Echevarria, I. Scimemi and A. Vladimirov, Unpolarized transverse momentum dependent parton distribution and fragmentation functions at next-to-next-to-leading order, JHEP 09 (2016) 004 [arXiv: 1604.07869] [INSPIRE].

[100] S. Moch, B. Ruijl, T. Ueda, J.A.M. Vermaseren and A. Vogt, Four-loop non-singlet splitting functions in the planar limit and beyond, JHEP 10 (2017) 041 [arXiv:1707.08315] [INSPIRE].

[101] A. Grozin, J. Henn and M. Stahlhofen, On the Casimir scaling violation in the cusp anomalous dimension at small angle, JHEP 10 (2017) 052 [arXiv:1708.01221] [INSPIRE].

[102] E. Laenen, G.F. Sterman and W. Vogelsang, Higher order QCD corrections in prompt photon production, Phys. Rev. Lett. 84 (2000) 4296 [hep-ph/0002078] [INSPIRE].

[103] A. Kulesza, G.F. Sterman and W. Vogelsang, Joint resummation in electroweak boson production, Phys. Rev. D 66 (2002) 014011 [hep-ph/0202251] [INSPIRE].

[104] G. Bozzi, S. Catani, D. de Florian and M. Grazzini, Transverse-momentum resummation and the spectrum of the Higgs boson at the LHC, Nucl. Phys. B 737 (2006) 73 [hep-ph/0508068] [INSPIRE].

[105] J.-W. Qiu and X.-F. Zhang, Role of the nonperturbative input in QCD resummed Drell-Yan $Q_{T}$ distributions, Phys. Rev. D 63 (2001) 114011 [hep-ph/0012348] [INSPIRE]. 
[106] I. Scimemi and A. Vladimirov, Power corrections and renormalons in transverse momentum distributions, JHEP 03 (2017) 002 [arXiv: 1609.06047] [INSPIRE].

[107] I. Scimemi and A. Vladimirov, Analysis of vector boson production within TMD factorization, arXiv:1706.01473 [INSPIRE].

[108] V.M. Braun, T. Lautenschlager, A.N. Manashov and B. Pirnay, Higher twist parton distributions from light-cone wave functions, Phys. Rev. D 83 (2011) 094023 [arXiv:1103.1269] [INSPIRE].

[109] Z. Ligeti, I.W. Stewart and F.J. Tackmann, Treating the $b$ quark distribution function with reliable uncertainties, Phys. Rev. D 78 (2008) 114014 [arXiv:0807.1926] [INSPIRE].

[110] R. Abbate, M. Fickinger, A.H. Hoang, V. Mateu and I.W. Stewart, Thrust at $N^{3} L L$ with power corrections and a precision global fit for $\alpha_{s}\left(m_{Z}\right)$, Phys. Rev. D 83 (2011) 074021 [arXiv: 1006.3080] [INSPIRE].

[111] C.F. Berger, C. Marcantonini, I.W. Stewart, F.J. Tackmann and W.J. Waalewijn, Higgs production with a central jet veto at NNLL+NNLO, JHEP 04 (2011) 092 [arXiv: 1012.4480] [INSPIRE].

[112] T. Gehrmann, T. Lübbert and L.L. Yang, Calculation of the transverse parton distribution functions at next-to-next-to-leading order, JHEP 06 (2014) 155 [arXiv:1403.6451] [INSPIRE].

[113] T. Gehrmann, A. von Manteuffel and L. Tancredi, The two-loop helicity amplitudes for $q \bar{q}^{\prime} \rightarrow V_{1} V_{2} \rightarrow 4$ leptons, JHEP 09 (2015) 128 [arXiv: 1503.04812] [INSPIRE].

[114] F. Caola, J.M. Henn, K. Melnikov, A.V. Smirnov and V.A. Smirnov, Two-loop helicity amplitudes for the production of two off-shell electroweak bosons in gluon fusion, JHEP 06 (2015) 129 [arXiv:1503.08759] [INSPIRE].

[115] S. Moch, J.A.M. Vermaseren and A. Vogt, The three loop splitting functions in QCD: the nonsinglet case, Nucl. Phys. B 688 (2004) 101 [hep-ph/0403192] [INSPIRE].

[116] A. Vogt, S. Moch and J.A.M. Vermaseren, The three-loop splitting functions in QCD: the singlet case, Nucl. Phys. B 691 (2004) 129 [hep-ph/0404111] [INSPIRE].

[117] M.A. Ebert, J.K.L. Michel and F.J. Tackmann, Resummation improved rapidity spectrum for gluon fusion Higgs production, JHEP 05 (2017) 088 [arXiv: 1702.00794] [INSPIRE].

[118] A.P. Bukhvostov, G.V. Frolov, L.N. Lipatov and E.A. Kuraev, Evolution equations for quasi-partonic operators, Nucl. Phys. B 258 (1985) 601 [INSPIRE].

[119] V.M. Braun, A.N. Manashov and J. Rohrwild, Renormalization of twist-four operators in QCD, Nucl. Phys. B 826 (2010) 235 [arXiv:0908.1684] [INSPIRE].

[120] J.C. Collins and M. Diehl, Transversity distribution does not contribute to hard exclusive electroproduction of mesons, Phys. Rev. D 61 (2000) 114015 [hep-ph/9907498] [INSPIRE].

[121] A. Bacchetta and A. Prokudin, Evolution of the helicity and transversity transverse-momentum-dependent parton distributions, Nucl. Phys. B $\mathbf{8 7 5}$ (2013) 536 [arXiv:1303.2129] [INSPIRE].

[122] D. Gutiérrez-Reyes, I. Scimemi and A.A. Vladimirov, Twist-2 matching of transverse momentum dependent distributions, Phys. Lett. B 769 (2017) 84 [arXiv:1702.06558] [INSPIRE]. 
[123] G. 't Hooft and M.J.G. Veltman, Regularization and renormalization of gauge fields, Nucl. Phys. B 44 (1972) 189 [inSPIRE].

[124] P. Breitenlohner and D. Maison, Dimensional renormalization and the action principle, Commun. Math. Phys. 52 (1977) 11 [inSPIRE].

[125] S.A. Larin, The renormalization of the axial anomaly in dimensional regularization, Phys. Lett. B 303 (1993) 113 [hep-ph/9302240] [INSPIRE].

[126] S. Moch, J.A.M. Vermaseren and A. Vogt, On $\gamma_{5}$ in higher-order QCD calculations and the NNLO evolution of the polarized valence distribution, Phys. Lett. B 748 (2015) 432 [arXiv: 1506. 04517] [INSPIRE].

[127] W. Vogelsang, The spin dependent two loop splitting functions, Nucl. Phys. B 475 (1996) 47 [hep-ph/9603366] [inSPIRE].

[128] Y. Matiounine, J. Smith and W.L. van Neerven, Two loop operator matrix elements calculated up to finite terms for polarized deep inelastic lepton-hadron scattering, Phys. Rev. D 58 (1998) 076002 [hep-ph/9803439] [INSPIRE].

[129] A. Weber, Soft gluon resummations for polarized Drell-Yan dimuon production, Nucl. Phys. B 382 (1992) 63 [InSPIRE].

[130] M. Stratmann, A. Weber and W. Vogelsang, Spin dependent nonsinglet structure functions in next-to-leading order, Phys. Rev. D 53 (1996) 138 [hep-ph/9509236] [INSPIRE].

[131] S. Moch, J.A.M. Vermaseren and A. Vogt, The three-loop splitting functions in QCD: the helicity-dependent case, Nucl. Phys. B 889 (2014) 351 [arXiv:1409.5131] [INSPIRE].

[132] V. Ravindran, J. Smith and W.L. van Neerven, NLO differential distributions of massive lepton pair production in longitudinally polarized proton proton collisions,

Nucl. Phys. B 647 (2002) 275 [hep-ph/0207076] [INSPIRE].

[133] W.A. Bardeen, A.J. Buras, D.W. Duke and T. Muta, Deep inelastic scattering beyond the leading order in asymptotically free gauge theories, Phys. Rev. D 18 (1978) 3998 [InSPIRE].

[134] G. Altarelli and G. Parisi, Asymptotic freedom in parton language, Nucl. Phys. B 126 (1977) 298 [INSPIRE].

[135] X. Artru and M. Mekhfi, Transversely polarized parton densities, their evolution and their measurement, Z. Phys. C 45 (1990) 669 [InSPIRE].

[136] J.-Y. Chiu, A. Jain, D. Neill and I.Z. Rothstein, The rapidity renormalization group, Phys. Rev. Lett. 108 (2012) 151601 [arXiv:1104.0881] [INSPIRE].

[137] J.-Y. Chiu, A. Jain, D. Neill and I.Z. Rothstein, A formalism for the systematic treatment of rapidity logarithms in quantum field theory, JHEP 05 (2012) 084 [arXiv:1202.0814] [INSPIRE].

[138] Y. Li, D. Neill and H.X. Zhu, An exponential regulator for rapidity divergences, arXiv: 1604.00392 [INSPIRE].

[139] T. Becher and M. Neubert, Drell-Yan production at small $q_{T}$, transverse parton distributions and the collinear anomaly, Eur. Phys. J. C 71 (2011) 1665

[arXiv: 1007.4005] [INSPIRE].

[140] M.G. Echevarría, A. Idilbi and I. Scimemi, Soft and collinear factorization and transverse momentum dependent parton distribution functions, Phys. Lett. B 726 (2013) 795 [arXiv:1211.1947] [INSPIRE]. 
[141] D. Binosi and L. Theussl, JaxoDraw: a graphical user interface for drawing Feynman diagrams, Comput. Phys. Commun. 161 (2004) 76 [hep-ph/0309015] [INSPIRE].

[142] D. Binosi, J. Collins, C. Kaufhold and L. Theussl, JaxoDraw: a graphical user interface for drawing Feynman diagrams. Version 2.0 release notes, Comput. Phys. Commun. 180 (2009) 1709 [arXiv:0811.4113] [inSPIRE].

[143] R. Mertig, M. Böhm and A. Denner, FeynCalc: computer algebraic calculation of Feynman amplitudes, Comput. Phys. Commun. 64 (1991) 345 [INSPIRE].

[144] V. Shtabovenko, R. Mertig and F. Orellana, New developments in FeynCalc 9.0, Comput. Phys. Commun. 207 (2016) 432 [arXiv:1601.01167] [INSPIRE].

[145] M. Sjödahl, ColorMath - a package for color summed calculations in $\mathrm{SU}\left(N_{c}\right)$, Eur. Phys. J. C 73 (2013) 2310 [arXiv:1211.2099] [INSPIRE].

[146] J.A.M. Vermaseren, New features of FORM, math-ph/0010025 [INSPIRE].

[147] J. Kuipers, T. Ueda, J.A.M. Vermaseren and J. Vollinga, FORM version 4.0, Comput. Phys. Commun. 184 (2013) 1453 [arXiv:1203.6543] [InSPIRE].

[148] A.V. Belitsky, Leading order analysis of twist-3 space- and time-like cut vertices in QCD, in Annual $31^{\text {st }}$ PNPI Winter School on Nuclear and Particle Physics, St. Petersburg Russia, 24 February-2 March 1997 [Int. J. Mod. Phys. A 32 (2017) 1730018] [hep-ph/9703432] [INSPIRE].

[149] J.C. Collins and D.E. Soper, Parton distribution and decay functions, Nucl. Phys. B 194 (1982) 445 [inSPIRE].

[150] J.C. Collins and T.C. Rogers, The gluon distribution function and factorization in Feynman gauge, Phys. Rev. D 78 (2008) 054012 [arXiv:0805.1752] [InSPIRE]. 